CLINICAL JUDGMENT FAITH BIAS: THE IMPACT OF FAITH AND MULTICULTURAL COMPETENCE ON CLINICAL JUDGMENT

\author{
A DISSERTATION \\ SUBMITTED TO THE COMMITTEE \\ IN PARTIAL FULFILLMENT OF THE REQUIREMENT \\ FOR THE DEGREE \\ DOCTOR OF PHILOSOPHY \\ BY \\ KEVIN A. HARRIS \\ DISSERTATION ADVISOR: DR. PAUL SPENGLER
}

BALL STATE UNIVERSITY

MUNCIE, IN

MAY 2011 


\title{
CLINICAL JUDGMENT FAITH BIAS: THE IMPACT OF FAITH AND MULTICULTURAL COMPETENCE ON CLINICAL JUDGMENT
}

\author{
A DISSERTATION \\ SUBMITTED TO THE GRADUATE SCHOOL \\ IN PARTIAL FULFILLMENT OF THE REQUIREMENT \\ FOR THE DEGREE \\ DOCTOR OF PHILOSOPHY
}

BY

KEVIN A. HARRIS

APPROVED BY:

Committee Chairperson

Date

Committee Member

Date

Committee Member

Date

Committee Member

Date

BALL STATE UNIVERSITY

MUNCIE, IN

MAY 2011 


\section{Table of Contents}

Table of Contents $\quad$ iii

List of Tables viii

List of Figures $\quad$ X

Acknowledgements $\quad$ xi

$\begin{array}{lll}\text { Abstract } & \text { xiv }\end{array}$

Chapter 1: Introduction $\quad 1$

$\begin{array}{lr}\text { Clinical Judgment Faith Bias } & 2\end{array}$

$\begin{array}{ll}\text { Faith Magnitude and Bias } & 4\end{array}$

$\begin{array}{ll}\text { Religion and Obsessionality } & 7\end{array}$

$\begin{array}{ll}\text { Evidence of Bias } & 9\end{array}$

Stereotyping, Salience Bias, and the Representativeness Heuristic $\quad 10$

Weaknesses in Previous Clinical Judgment Faith Bias Studies 11

$\begin{array}{ll}\text { Religiousness and Spirituality } & 12\end{array}$

Clinician Individual Differences in Clinical Judgment Faith Bias $\quad 16$

$\begin{array}{ll}\text { Clinician multicultural competence } & 17\end{array}$

$\begin{array}{ll}\text { Clinician magnitude of personal faith } & 19\end{array}$

$\begin{array}{ll}\text { Clinician view of faith helpfulness } & 20\end{array}$

$\begin{array}{ll}\text { Purpose } & 22\end{array}$

$\begin{array}{ll}\text { Hypotheses } & 22\end{array}$

$\begin{array}{ll}\text { Faith bias hypotheses } & 22\end{array}$

Resilience hypothesis $\quad 24$ 
$\begin{array}{ll}\text { Summary } & 26\end{array}$

Chapter 2: Literature Review 29

Religious and Spiritual Values in Clinical Work 30

Clinical Judgment Faith Bias $\quad 36$

$\begin{array}{ll}\text { Religion and Psychopathology } & 39\end{array}$

Clinical Judgment Faith Bias and Faith Magnitude 42

$\begin{array}{ll}\text { Moderate Religiousness } & 47\end{array}$

Religiousness, Faith Magnitude, and Psychopathology 50

Religion and Obsessionality $\quad 57$

$\begin{array}{ll}\text { Evidence of Bias } & 61\end{array}$

Stereotyping, Salience Bias, and the Representativeness Heuristic 62

Weaknesses in Previous Clinical Judgment Faith Bias Studies 66

Client Religiousness, Spirituality, and Clinical Judgment Faith Bias $\quad 68$

$\begin{array}{ll}\text { Clinician Multicultural Competence and Faith Bias } & 77\end{array}$

Clinician Magnitude of Personal Faith and Faith Bias $\quad 82$

Clinician View of Faith Helpfulness $\quad 87$

$\begin{array}{ll}\text { Summary } & 90\end{array}$

$\begin{array}{ll}\text { Chapter 3: Method } & 94\end{array}$

$\begin{array}{ll}\text { Participants } & 95\end{array}$

$\begin{array}{ll}\text { Demographics } & 95\end{array}$

$\begin{array}{ll}\text { Power analysis } & 97\end{array}$

$\begin{array}{lr}\text { Case Vignette } & 99\end{array}$ 
The Multicultural Counseling Knowledge and Awareness Scale (MCKAS)

The Spiritual - Religious Orientation Scale (S-ROS)

Religious Issues in Therapy Survey Form (RITSF)

Procedure

Chapter 4: Results

Regression Diagnostics

Data Analyses

Summary

Findings

Limitations

Research Implications

Clinical Implications

Conclusion

Appendix A: Participant Demographic Characteristics 
Appendix D: Survey Booklet 209

Appendix D1: Case Vignette 210

Appendix D2: The Schedule of Therapists' Prognostic

Expectations (STPE)

Appendix D3: The Multicultural Counseling Knowledge and

Awareness Scale (MCKAS)

Appendix D4: The Spiritual - Religious Orientation Scale

(S-ROS)

Appendix D5: The Religious Issues in Therapy Survey Form (RITSF)

Appendix D6: Demographic Questions

Appendix D7: Debriefing Information

Appendix E: Survey Correspondence

Appendix E1: Prenotice Letter

Appendix E2: Survey Cover Letter

Appendix E3: Thank You Letter Text

Appendix E4: Nonrespondent Mailing Cover Letter

Appendix E5: Priority Mail Special Contact Letter

Appendix F: Six Different Versions of Vignette Paragraph 2 Used in the

Current Study

Appendix G: Vignette Development Procedure

Appendix H: Pilot Study 1 Questionnaire (Clinician Questionnaire) 
Appendix J: Pilot Study 2 Questionnaire (Religious/Spiritual Expert

Raters Questionnaire)

Appendix K: Pilot Study 3 Questionnaires (Magnitude Estimation Scaling Pilot Study)

Appendix L: Factor Analysis Data

Appendix L1: S-ROS Structure Matrix

Appendix L2: S-ROS Inter-Item Correlation Matrix

Appendix L3: RITSF Structure Matrix

Appendix L4: RITSF Inter-Item Correlation Matrix

Appendix L5: STPE Structure Matrix

Appendix L6: STPE Inter-Item Correlation Matrix

Variable Transformations

Missing Data

Multicollinearity

Measurement Error

Specification Error

Normality

Nonlinearity 360

Heteroscedasticity

Outliers 


\section{List of Tables}

Table 1: Magnitude Estimation Scaling Calculations

Table 2: Means $(M)$, Standard Deviations $(S D)$, and Cronbach's Alphas $(\alpha)$ for All Scales Completed by Participants

Table 3: Bivariate Correlations Among All Dependent and Independent

Variables

Table 4: Regression 1

Table 5: Regression 2

Table 6: Regression 3

Table 7: Regression 4

Table 8: Regression 5

Table 9: Main Effect of Client/Vignette Religiousness on Clinical Judgments of

Pathology From Previous Research

Table 10: Interaction Effect of Client/Vignette Religiousness and Clinician Religiousness on Clinical Judgments of Pathology From Previous Research

Table 11: Psychologist Expert Raters Diagnostic Ratings

Table 12: Mean Accuracy Ratings of 37 Vignette Versions

Table 13: Magnitude Estimation Scaling Calculations

Table 14: Tolerance, Variance Inflation Factor (VIF), and Condition Index (CIndex) for Each Variable Regressed on Remaining Variables 
Table 15: Adjusted $R^{2}$ Values for Planned Regressions and Regressions with Variables Deleted

Table 16: Skewness and Kurtosis of Each Variable

Table 17: Ten Highest Values for Mahalanobis Distances, Leverage, and Cook's $D$ 


\section{List of Figures}

Figure 1: Pathology ratings of religious and spiritual cases at increasing magnitudes of client faith

Figure 2: Pathology ratings of religious and spiritual cases at increasing magnitudes of client faith by clinicians with low, moderate, and high levels of any one clinician attribute

Figure 3: Frequency histograms for each variable

Figure 4: Scatterplots of bivariate relationships among variables

Figure 5: Regression standard predicted values plotted against regression standardized residuals for prognosis and diagnosis

Figure 6: Normal P-P plots of regression standardized residuals for prognosis and diagnosis 


\section{Acknowledgements}

There are so many people to whom I owe thanks in making this monumental undertaking possible.

First and foremost I must extend my deepest gratitude to Dr. Spengler, for guiding me painstakingly through the arduous process of topic selection, literature review, design and methodology, many hypothesis revisions, 3 pilot studies, data collection, data analysis, data reanalysis, writing, rewriting, and more rewriting. You have been there for me every step along the way. Without you, this project would have taken a much different form, and I owe you many thanks for pushing me to go above and beyond - and for wading laboriously through my verbose writing.

I would like to thank Dr. Dixon, Dr. Alexander, Dr. Horowitz, and Dr. CorbettHemeyer, for plodding through the process with me, and having patience with me through my epic journey.

I must thank Dr. Schneider for giving me guidance in analyzing my data, helping me navigate my way through an immensely complex set of analyses, and helping me long after you were my stats professor.

I must thank Dr. Gollery for helping me re-analyze my data and volunteering to read my mammoth text for clarity. I am grateful to you for giving me a sounding board for talking through the ambitious set of analyses I performed, providing me with helpful feedback, helping me to see that I do understand the rationale for the statistical analyses I performed and can defend it conceptually, and giving me the inspiration to get through the home stretch of my research. 
I must thank Nicole for wrestling with my writing and spending many long hours editing my gargantuan thesis. I must also thank you for putting up with two long years of late nights and early mornings working on this project, analyzing and reanalyzing my data, waiting and hoping for it to be finally done, ignoring you, taking advantage of your editing acumen, bouncing ideas off you which you sometimes scarcely understood but always listened to - and agreeing to marry me anyway. I owe you, Ciara, Abigail, Fenway, and Mittens many, many thanks.

I must thank my parents, Scott, and the rest of my family for putting up with eight long years of "I'm working on it" and wondering when I'll finally graduate, for patiently waiting and supporting me through nearly a decade of academic labor even after I got my Masters degree. Scott, how ironic that we got to graduate at about the same time.

I would like to thank Spike for all your years of friendship and support, and for inspiring me to choose spirituality as my dissertation topic, listening to my many ideas and ramblings, and inspiring me to reach the unreachable stars. You almost caught up to me. Good luck on finishing your own dissertation, my friend.

I would like to thank Markov for your support and encouragement, for allowing me to bounce ideas off you and pick your brain on methodology and statistics. I would also like to thank Markov, Terry, Vance, Steph, Deniz, Khush, Jui, and Shonali, for giving me hope that graduation is possible, and I must thank Rashaana, Tanya, Melina, Marianna, and Eva for helping to keep me on track during Internship.

I would like to thank Dr. Spengler, Dr. Dixon, Pastor Raymond, Reverend Jackson, Dr. Barton, Dr. Stanford, Dr. Vandenbark, Dr. Hill, Dr. Iozzio, Dr. Carter Waren, Dr. Hutchingson, Pastor Gilbert, and Reverend Perchlik for your help in 
developing the 37 religious and spiritual vignette versions, and I would like to thank Jamalat and Dr. Spengler for your help in the magnitude estimation scaling of the final 14 versions, as well as the 47 undergraduates who participated in the magnitude estimation scaling task. I would also like to thank Dr. Spengler, Dr. Dixon, Dr. Rivas-Vasquez, Dr. Bowman, Dr. Nicolas, Dr. Rosen, Dr. Davis, Georgios, Khush, Markov, Steph, Rashaana, Marianna, Genelle, Amanda, and Eric for your help in developing the GAD vignette paragraph.

Finally, I owe a debt of gratitude to the 400 psychologists in my national sample for considering participating in my study, and to the APA Research Office for providing them. I am especially grateful to the 144 psychologists who took the time and effort to fill out my primary survey. To you I am greatly indebted. 


\begin{abstract}
Clinical judgment faith bias is a hypothesized tendency for clinicians to make more pathological judgments for clients with higher magnitudes of faith than for otherwise identical clients with socially normative faith. To test for clinical judgment faith bias, Dillman's (2007) Tailored Design method for mail and internet surveys was employed. A random sample of 141 psychologists in clinical practice completed a series of questionnaires measuring clinician religiousness and spirituality, view of faith helpfulness, multicultural awareness and knowledge, and diagnostic and prognostic judgments of a clinical vignette describing a client with Generalized Anxiety Disorder and two manipulated attributes: magnitude of faith (low, moderate, or high) and type of faith (religious or spiritual). Five multivariate multiple regression analyses were conducted. The results of the analyses were not statistically significant, so none of the planned post hoc analyses were conducted. The magnitude of faith in the vignette did not influence the diagnostic or prognostic judgments of clinicians, clinicians did not make significantly different judgments for religious cases than for spiritual cases, and faith magnitude did not interact with faith type. Furthermore, the various clinician attributes did not appear to moderate these clinical judgments. Implications are discussed for clinical judgment research and practice.
\end{abstract}




\section{Chapter 1}

\section{Introduction}

Values are an important component of psychotherapy (Worthington, 1986). Both clinicians and clients have values, and psychotherapy itself has its own set of values which inform how mental problems are understood and treated. Most clinicians treat their clients' values with sensitivity and respect (Belaire \& Young, 2002), and most do not let their own beliefs interfere with providing effective therapeutic treatment (Spengler, Strohmer, Dixon, \& Shivy, 1995). In successful therapy, however, clients often change their values to be more in line with those of their counselors (Worthington, Kurusu, McCullough, \& Sandage, 1996). Clinicians, then, must be mindful of the role their implicit or explicit values play in counseling so they may make sound clinical judgments and provide competent treatments.

Clinicians must be particularly mindful of people's faith values, particularly religiousness and spirituality. They constitute a special set of values which often arise in

counseling (Rose, Westefeld, \& Ansely, 2001). Even when issues of faith are not a focus of therapy, religion and spirituality are elements of cultural diversity which clinicians often encounter in their clinical practices (Frame, 2003). While most therapists are 
willing to discuss faith in the therapeutic process (e.g., Bergin, 1991), individuals who identify as being religious or spiritual often believe most counselors to be secular; they expect their counselors to ignore, minimize, or even pathologize their religious beliefs (Worthington, 1986). Historically, psychology as a profession has exhibited bias against religion (Houts \& Graham, 1986). Many psychologists view religion as a competing worldview or as being irrelevant, irrational, or pathological (Boadella, 1998; Cornett, 1998; Khalili, Murken, Reich, Ali Shah, \& Vahabzadeh, 2002). Likewise, religious leaders have often viewed scientific, psychological thinking as conflicting with religious teachings (Cornett, 1998). Psychology and religion are based on different epistemological assumptions. Clinicians usually do not receive specific training for religious or spiritual issues, and many psychologists believe only clergy and others with specific religious training should address religious concerns (Cornett, 1998; Frame, 2003). Furthermore, a religiousness gap (Bergin, 1980) exists between psychologists and the general population: psychologists tend to be less religious than the general population, which makes it more likely these clinicians may view issues of faith as irrelevant and possibly deviant.

\section{Clinical Judgment Faith Bias}

Some clinical judgment studies suggest clinicians occasionally make biased clinical judgments of highly religious clients. Despite the fact that the mental health literature has shown base rates of psychopathology are not significantly higher among religious people than among nonreligious people (e.g., Miller \& Kelley, 2005), clinicians still make different and often more pathological diagnostic or prognostic judgments for highly religious clients than for secular clients with identical symptoms (Gartner, 
Harmatz, Hohmann, Larson, \& Gartner, 1990a, 1990b; Hillowe, 1985; Houts \& Graham, 1986; Jones, 1991; Lewis \& Lewis, 1985; Marcinkowski, 1993; O’Connor \& Vandenberg, 2005; Sanderson, Vandenberg, \& Paese, 1999). Hillowe (1985), for example, reported that therapists perceive religious clients as being less appropriate for counseling. Houts and Graham (1986) found clinicians rated moderately religious clients as having more psychopathology and a worse prognosis than very religious or nonreligious clients. Sanderson, Vandenberg, and Paese (1999) reported that mental health professionals made clinical judgments based on the degree the religious content of case vignettes deviated from the norm. Similarly, O'Connor and Vandenberg (2005) discovered mental health professionals gave higher pathology ratings to members of less mainstream religions than to members of more mainstream religions. Finally, Gartner and colleagues (1990a, 1990b) found clinicians rated clients with extreme (religious or political) ideologies as having more severe psychopathology than clients with moderate beliefs.

A misconception exists associating religiousness with psychopathology, though there is no empirical evidence linking religiousness with a greater occurrence of mental health problems. In fact, much of the evidence points to the opposite conclusion.

Measures of religious involvement are positively associated with mental health outcomes, suggesting people who are religious may be more mentally healthy than their secular counterparts (Koenig, 1998, 2005; Miller \& Kelley, 2005; Oman \& Thoresen, 2005). Research to date nevertheless suggests some clinicians do make different and often more pathological clinical judgments, either more disabling diagnoses or more pathological prognoses, for clients who are very religious than for clients who are moderately religious 
or nonreligious (Gartner et al., 1990a, 1990b; Houts \& Graham, 1986; O’Connor \& Vandenberg, 2005). Because these clinical judgments disregard relevant base rates of pathology among religious clients, they represent a type of bias referred to in this study as clinical judgment faith bias. Clinical judgment faith bias refers to a hypothesized tendency for clinicians to make more pathological clinical judgments for clients with higher magnitudes of faith than for otherwise identical clients with what might be considered to more socially normative faith.

\section{Faith Magnitude and Bias}

The extant literature on this bias suggests biased clinical judgments of religious clients may be more related to the magnitude of client faith than to the actual religious content of their beliefs and practices. High magnitudes of religiousness are considered to be socially deviant, violating social norms by falling outside the bounds of what mainstream society considers to be typical and normal (Miller \& Kelley, 2005; Momen, 1999). Social deviance is often believed to be associated with psychopathology (Barlow \& Durand, 1995). Some socially deviant traits are associated with psychopathology, such as criminal behavior, drug use, elevated levels of arrest, and sexual promiscuity (Dishion, 2000). Psychologists may be particularly prone to equate social deviance and psychopathology, because there is a general clinician bias to diagnose psychopathology

(Garb, 1998). Hence, psychologists may be more likely to perceive socially deviant religious beliefs and behaviors as psychopathological. Furthermore, because a stereotype exists among psychologists associating religiousness with psychopathology (Ellis, 1980; Freud, 1907/1959, 1927; Miller \& Kelley, 2005; Richards \& Bergin, 2000), salient socially deviant forms of religiousness such as highly religious behavior may activate this 
stereotype and make it more likely psychologists will conclude highly religious behaviors are symptoms of mental problems. But does evidence exist to associate high magnitudes of religiousness with psychopathology?

In a few cases, there is empirical evidence to support the contention that high magnitudes of religious expression are associated with psychopathology. For example, some studies have shown religious extremists, cult members (Frame, 2003), young adult Pentecostals (Koenig, George, Meador, Blazer, \& Dyck, 1994), and rapid religious converts (Miller \& Kelley, 2005) often have increased rates of mental health problems. With the exception of the young adult Pentecostals, though, these forms of religious expression may not represent authentic forms of religiousness - that is, people who are members of traditional religions might not view these alternative forms of religiousness to be genuine expressions of their own faith (Sanderson et al., 1999). Sanderson et al. (1999) found a positive correlation between conventional religious practices, authenticity, and mental health; mental health professionals judged people with unconventional religious practices as having less authentic religious experiences and as being less mentally healthy than people with conventional forms of religious expression.

When inauthentic forms of religious expression are excluded, however, there is little evidence to support a direct association between high magnitudes of religiousness and psychopathology. Longitudinal data from Lindenthal, Myers, Pepper, and Stern (1970) point to an inverse, “dose-response” relationship between religious affiliation and psychological impairment, and decades of empirical research reviewed by Koenig (1998, 2005) report strong positive relationships between religious involvement and mental health. Although the majority of studies indicate religion has a positive influence on 
mental health, applicable studies exist for every major diagnostic category in the Diagnostic and Statistical Manual of Mental Disorders ( $4^{\text {th }}$ edition, text revision; American Psychiatric Association, 2000) showing a positive correlation between religion and psychopathology (Koenig, 1998, 2005; Miller \& Kelley, 2005). As Miller and Kelley (2005) pointed out, "in teasing apart the multiplicity of influences on mental health, it quickly becomes clear that a consistent, robust, and unidirectional relationship between mental health and religiousness or spirituality is an illusion - the reality is far more subtle and complex" (p. 462).

Furthermore, when religiousness is examined as a continuous characteristic instead of as a categorical quality (as many studies have represented it), a curvilinear relationship sometimes appears between religiousness and psychopathology. Miller and Kelley (2005) observed that a close examination of correlational data suggests a curvilinear relationship between religiousness and overall distress (Ross, 1990), depressive expression (Schnittker, 2001), general anxiety (Koenig, Ford, et al., 1993), death anxiety (Pressman, Lyons, Larson, \& Gartner, 1992), and mental health over the course of the lifespan (Ingersoll-Dayton, Krause, \& Morgan, 2002). In some cases, high and low levels of religiousness are both correlated with better mental health, while moderate levels of religiousness are more highly correlated with indicators of poor mental health, such as fear of death (Wink \& Scott, 2005), depression (Eliassen, Taylor, \& Lloyd, 2005), and racism (Perkins, 1992). Thus highly religious people may actually have lower base rates of mental health issues than moderately religious people do, and the same base rates of mental illness as are found among their nonreligious counterparts. In other cases, "those at the far ends of the religious continuum are the most susceptible 
to suffering, and those that adhere to a more moderate practice or belief system show better functioning" (Miller \& Kelley, 2005, p. 462). Overall, the empirical literature provides some evidence to support the idea that people who are highly religious are more likely to experience psychopathology, but it also provides considerably more evidence to the contrary. It would be prudent, therefore, for future studies of religious clinical judgments to differentiate between different magnitudes of religiousness, and to measure this variable as continuous.

\section{Religion and Obsessionality}

Since Freud, a stereotype has existed among clinicians and lay persons alike equating religious behaviors with obsessional symptoms signifying mental problems (Greenberg, 1984; Tek \& Ulug, 2001; Yossifova \& Loewenthal, 1999). In fact, the term religious scrupulosity evolved to describe symptoms of obsessive compulsive disorder involving pathological religious obsessions and compulsions emphasizing ritual-based purity (Greenberg \& Witztum, 1994; Miller \& Kelley, 2005). Recent research suggests clinicians may be likely to exhibit the hypothesized clinical judgment faith bias in evaluating religious clients with anxiety disorders (Gartner et al., 1990a, 1990b; Yossifova \& Loewenthal, 1999). Gartner and colleagues (1990a, 1990b) found when clinicians were asked to give diagnoses for nearly equivalent cases, clients with extreme ideologies (either religious or political) were almost three times more likely to be diagnosed with obsessive compulsive disorder (OCD) than clients with less extreme ideologies, but were less likely to be diagnosed with generalized anxiety disorder (GAD). This is surprising, because the prevalence of OCD in the general population $(2.5 \%)$ is half that of GAD, according to the DSM-IV-TR (5\%; American Psychiatric Association, 
2000). Similarly, Yossifova and Loewenthal (1999) found lay judges who were shown cases with OCD symptoms judged the cases as having more obsessional symptoms and worse psychological problems when these cases described highly religious people than when they described people with identical symptoms who were low in religiousness. Taken together, these two studies suggest a stereotype associating religious behaviors and obsessional symptoms.

There is mixed evidence to support the veridicality of this stereotype. Several studies investigating the relationship between religiousness and OCD have found no relationship between religious beliefs and any clinical feature of OCD, including religious obsessions (Lewis, 1998; Tek \& Ulug, 2001; Yaryura-Tobias, Grunes, Todaro, McKay, Neziroglu, \& Stockman, 2000). Some reviews on religion and mental health (e.g., Harris, Schoneman, \& Carrera, 2002; Miller \& Kelley, 2005) reported a small positive correlation between general symptoms of anxiety and religion, but the relationship between these variables disappeared when socioeconomic status, social support, disability, and chronic illness were controlled for (Koenig, Ford, George, Blazer, \& Meador, 1993; Koenig, George, Blazer, Pritchett, \& Meador, 1993). Most reviews have found negative or no correlation between religiousness and anxiety in general (59 studies cited in Koenig, 1998) or between religiousness and OCD in particular (Hermesh, Masser-Kavitsky, \& Gross-Isseroff, 2003; Greenberg \& Shefler, 2002; Tek \& Ulug, 2001; Okasha, Lotaief, Ashour, El Mahalawy, El Dawla, \& El-Kholy, 2000). Furthermore, while some authors have speculated OCD is more common in highly religious societies (Chackraborty, 1975), Greenberg and Shefler (2002) noted similar 
prevalence rates for OCD in highly religious and nonreligious countries, indirectly arguing against this contention.

Some studies, however, have found an association between religiousness and obsessionality. For example, Steketee, Quay, and White (1991) found a significant positive correlation between religious identification and severity of OCD symptoms in a sample of OCD patients, and Abramowitz, Deacon, Woods, and Tolin (2004) found highly religious undergraduates reported more symptoms of obsessionality than moderately religious or atheist students. Similarly, Alonso and colleagues (2001) and Mataix-Cols and colleagues (2002) found patients with higher levels of obsessions with religious content to have poorer outcomes from treatment even after controlling for initial symptom severity (Alonso, Menchon, Pifarre, Mataix-Cols, Torres, Salgado, \& Vallejo, 2001; Mataix-Cols, Rosario-Campos, \& Leckman, 2002). When taking gender into consideration, however, Lewis and Maltby (1995) found obsessional characteristics associated with frequency of religious behavior and favorable attitude toward religion in females only. Koenig (2005) also pointed out religious participants might be expected to score higher than nonreligious participants on OCD scales, since many of these scales include questions which assess traditional religious values such as personal responsibility and attention to detail. Thus, an association between obsessionality and religion has only been found in a few cases and may be due to measurement bias rather than any genuine correlation.

\section{Evidence of Bias}

Nevertheless, the Gartner et al. (1990a, 1990b) and Yossifova and Loewenthal (1999) studies cited above found evidence OCD symptoms are more likely to be 
perceived in very religious people than in nonreligious people who have the same number and type of anxiety-related symptoms. This evidence indicates clinical judgments of psychologists may be influenced by the stereotype that religious behaviors are

obsessional symptoms signifying mental health problems. Combining this stereotype with the stereotype that any form of social deviance is a symptom of psychopathology, and with the misconception that religion is also a symptom of mental illness, this supports the distinct possibility psychologists may be more prone to experiencing clinical judgment faith bias when evaluating highly religious clients with obsessional symptoms.

\section{Stereotyping, Salience Bias, and the Representativeness Heuristic}

Behavior decision-making theory provides the underlying theory for why this hypothesized clinical judgment faith bias may occur. Specifically, stereotyping, salience bias, and the representativeness heuristic may explain why clinical judgment faith bias is more likely under certain circumstances. As noted previously, there are several stereotypes linking socially deviant behaviors and religious practice to psychopathology, including the erroneous notions that religion is associated with psychopathology (Miller \& Kelley, 2005), that deviance is related to psychopathology (Barlow \& Durand, 1995), and that religion is linked to obsessional symptoms (Yossifova \& Loewenthal, 1999). Salience bias occurs when clinicians make attributions based on the perceptual information which is most distinctive or memorable rather than the data which is most pertinent to the decision at hand (Taylor, 2006). The representativeness heuristic is a cognitive shortcut people use when judging whether an object or event is representative of a particular category or group without considering the base rates at which that object or event occurs in that group (Baron \& Byrne, 1997; Garb, 1998; Kahneman \& Tversky, 
1973; Nisbett \& Ross, 1980). In conditions where the aforementioned stereotypes are salient and readily available to clinicians (i.e., when they are evaluating highly religious clients with obsessional symptoms), salience bias may bring these stereotypes to mind, and clinicians may readily equate highly religious descriptors with symptoms of psychopathology. Those clinicians who invoke the representativeness heuristic may automatically access these stereotypes of highly religious people, without taking into consideration base rates showing no overall difference in rates of psychopathology for highly religious people when their faith is authentic (Kahneman \& Tversky, 1973; Nisbett \& Ross, 1980). These clinicians are then likely to engage in the aforementioned clinical judgment faith bias. Any one of these phenomena in isolation may be insufficient to lead to bias, but in situations in which all three cognitive heuristics come into play, stereotyping, salience bias, and the representativeness heuristic may work in concert with one another to lead to clinical judgment faith bias.

\section{Weaknesses in Previous Clinical Judgment Faith Bias Studies}

The few empirical studies investigating clinical judgment faith bias have had numerous weaknesses. First, many of them examined merely the presence or absence of religious ideation or behavior, rather than differing levels of religiousness (e.g., Gartner et al., 1990a, 1990b; Gerson, Allen, Gold, \& Kose, 2000; Hillowe, 1985; Lewis \& Lewis, 1985; Marcinkowski, 1993). Second, those studies examining different levels of religiousness in an effort to examine the magnitude of client faith often examined different levels of qualitatively different types of religious expressions (e.g., Catholic, Latter Day Saint, and Nation of Islam in O'Connor \& Vandenberg, 2005), rather than different levels of the same kind of faith. Additionally, some of these previous studies 
investigated forms of faith so extreme as to be artificial, inauthentic fabrications of faith not represented in the real world. For example, the "Unconventional" religious practices described in Sanderson et al. (1999) included giving away all of their children's possessions, having a vision in which the person is told they are Christ, hearing the voice of God telling the person to sacrifice their child, cutting off their hand after reading a Biblical passage to "cut off the hand that has sinned," and experiencing the presence of Elvis. Arguably, it is likely few religious adherents would consider these practices to be authentic expressions of their faiths.

Another weakness in many previous investigations of clinical judgment faith bias is their neglect of client spirituality. Client spirituality and client religiousness represent distinct constructs which may have different effects on any clinical judgment faith bias that does exist (Feeser, 1997; Zinnbauer \& Pargament, 2005). Examining spirituality and religiousness as independent constructs would improve upon the accuracy of existing estimates of clinical judgment faith bias, yet very few studies have treated these constructs as distinct variables.

\section{Religiousness and Spirituality}

There is growing recognition religiousness and spirituality may be discrete constructs. Numerous authors have used these terms to refer to concepts which are related and overlapping, but nevertheless distinct (Beck, 1986; Booth, 1984; Elkins, Hedstrom, Hughes, Leaf, \& Saunders, 1988; Ingersoll, 1994; Legere, 1984; Shafranske \& Gorsuch, 1984; Shafranske \& Malony, 1990; Turner, Lukoff, Barnhouse, \& Lu, 1995; Zinnbauer \& Pargament, 2005). In 1997, a group of 60 psychologists, medical doctors, neuroscientists, and substance abuse counselors convened for a series of conferences in which they 
attempted to define the terms religiousness and spirituality. Based on this conference series, Hill, Pargament, Hood, McCullough, Swyers, Larson, and Zinnbauer (2000) proposed definitional criteria for future research, a set of criteria for judging operational definitions of spirituality and religiousness. Paraphrased, Hill et al.’s (2000) working definition of spirituality was a search for the sacred, and their working definition of religiousness was a search for the sacred (or non-sacred things) in a socially-sanctioned context that typically facilitates searches for the sacred, where the sacred is an ultimate, transcendent, or divine being, reality, or truth as perceived by an individual.

Several years later, Zinnbauer and Pargament (2005) reviewed the historical trends in psychologists' attempts to define these two constructs, and each author presented his own set of definitions reflecting two overall trends in the literature. Zinnbauer defined spirituality as a personal or group search for the sacred and religiousness as a search for the sacred that unfolds within a traditional sacred context, conceptualizing religiousness as a contextualized or organized form of spirituality. This reflected a major trend in the recent psychology of religion literature defining spirituality as the broader construct. Pargament defined spirituality as a search for the sacred and religiousness as a search for significance in ways related to the sacred, conceptualizing religiousness as having a broader set of ends than spirituality, reflecting a major trend in the historical literature defining religiousness as the broader construct. Zinnbauer's definitions echoed Hill et al.'s (2000) definitional criteria.

Furthermore, a growing body of empirical research has provided evidence spiritual and religious scales measure distinct constructs. For example, in a meta-analysis of the relationship between the five-factor model (FFM) of personality and measures of 
religiousness and spirituality, Saroglou (2002) found religiousness scales appear to relate more to the FFM dimensions of Conscientiousness and Agreeableness, while spirituality scales seem to be more related to the FFM dimensions of Openness and Agreeableness. This suggests that while religiousness and spirituality share something in common, namely, "a compassionate attitude toward others," religiousness involves "more of the dutiful, procedural aspects of faith involvement," while spirituality involves "a seeking, curious attitude toward the transcendent" (Piedmont, 2005, p. 263). Similarly, in a joint factor analysis of the FFM and the Brief Multidimensional Measure of Religiousness/ Spirituality, Piedmont, Ciarrocchi, Dy-Liacco, Mapa, and Williams (2003) found religiousness and spirituality were highly correlated factors which were distinct from one another and from the five factors of personality.

Here, a close adaptation of Hill et al.'s (2000) working definitions of spirituality and religiousness have been adopted, because of the way in which they were developed, the number of prominent psychology of religion experts involved in their development, their comprehensiveness, and their similarity to other accepted definitions of these terms (e.g., Zinnbauer \& Pargament, 2005):

Religiousness is a search for the sacred (or non-sacred things such as social contact or appearing virtuous to your neighbors) in a traditional institutional context that typically facilitates searches for the sacred.

Spirituality is a personal search for the sacred which may or may not occur in a traditional institutional context that typically facilitates searches for the sacred. 
Again, the sacred is an ultimate, transcendent, or divine being, reality, or truth as perceived by an individual.

The clinical judgment literature on religion and spirituality is not instructive as to whether client spirituality as distinct from client religiousness affects the diagnostic or prognostic judgments of clinicians. Client spirituality may lead to a similar bias as client religiousness: clinicians may show a clinical judgment faith bias with both highly spiritual and highly religious clients. Returning to the issue of social deviance, clinicians may consider spirituality to be more socially acceptable, or less socially deviant, than religiousness (Bergin, 1991; Kahle, 1998). Internalized spiritual beliefs in the absence of religious rituals or practice may be viewed as being more normative than highly religious behavior. Alternately, internalized spiritual beliefs are difficult to observe behaviorally, in contrast to overt displays of religious ritual. If either of these are the case, then spirituality may be less likely to call religious stereotypes to mind and less likely to lead to salience bias, and clinicians may be less likely to invoke the representativeness heuristic with highly spiritual clients than with highly religious clients. Therefore, clinicians may show less clinical judgment faith bias with spiritual clients than with religious clients. Feeser's (1997) dissertation specifically examined counseling psychologists' ratings of the perceived attractiveness and overall prognosis of religious, spiritual, and secular cases, finding client spirituality as distinct from client religiousness did not affect prognostic judgments. Feeser (1997) did not examine diagnostic judgments, however. With the exception of Feeser's 1997 study, spirituality has rarely been measured as a construct separate from religiousness in the clinical judgment literature. Thus, it is unclear on the basis of one unreplicated study how spirituality as distinct from 
religiousness might influence the judgments clinicians make. If clinicians react differently to spiritual clients than they do to religious clients, this may indicate clinicians are reacting to the content of client faith. If clinicians respond to highly spiritual clients in the same way as they do to highly religious clients, this supports the notion that clinicians are reacting to the magnitude of client faith rather than its content. If, on the other hand, clinicians react differently to highly spiritual clients than they do to highly religious clients but react more to all clients as the magnitude of their faith increases, this too may indicate clinicians are reacting to the magnitude of client faith. Assessing spirituality and religiousness as distinct constructs would improve upon the accuracy of existing estimates of clinical judgment faith bias. Thus these constructs should be treated as distinct variables.

\section{Clinician Individual Differences in Clinical Judgment Faith Bias}

Finally, some clinician individual differences may make certain clinicians resilient, or less susceptible, to the hypothesized clinical judgment faith bias. Not all counselors may be equally susceptible to making biased religious and spiritual clinical judgments because of individual differences in the way they process clinical information. Specifically, clinician multicultural competence, magnitude of personal faith, and view of faith helpfulness may have moderating effects on any clinical judgment faith bias that exists (Kahle, 1998; Lewis \& Lewis, 1985; Worthington et al., 1996). These clinician attributes warrant examination in the interest of identifying clinician individual differences associated with resilience to bias, which may have implications for future clinical training. 
Clinician multicultural competence. Clinician multicultural competence may have a moderating effect on any clinical judgment faith bias that exists. In the Handbook of Multicultural Counseling, Jackson (1995) conceptualizes multicultural counseling as "counseling that takes place between or among individuals from different cultural backgrounds" (p. 3). Broadly defined, the term multicultural competence refers to those beliefs and attitudes, knowledge, and skills which lead to an awareness of one's own cultural values, the worldviews of others, and appropriate interventions for people from different cultures (Sue, Bernier, Durran, Feinberg, Pederson, Smith,, \& Vasquez-Nuttall, 1982; Sue, Arredondo, \& McDavis, 1992), where the term culture refers to race, ethnicity, nationality, age, gender, sexual orientation, religion, language, geographic region, immigration status, and disability (Pederson, 1990; Ponterotto, Casas, Suzuki, \& Alexander, 2001).

Multiculturally competent counselors may be less likely than other counselors to make clinical judgments influenced by race bias (Constantine, 2002; Ponterotto et al., 2001; Richeson \& Nussbaum, 2004). In other words, they have racial competence: they are competent in dealing with the cultural issue of race. Their racial competence has a moderating effect on racial clinical judgment biases whereby they are less likely to make judgments reflecting a race bias.

Many psychologists, counselors, and other scholars believe faith is an important part of multicultural competence (e.g., Feeser, 1997; Frame, 2003; Hall, 2001; Kelly, 1995; McLennan, Rochow, \& Arthur, 2001; Pate \& Bondi, 1992; Pederson, 1990; Richards \& Bergin, 2000). If this is the case, then multiculturally competent counselors may be less likely than other counselors to make decisions which are influenced by 
clinical judgment faith bias. They may have faith competence: competence in dealing with religious and spiritual issues in counseling. Their faith competence may have a moderating effect on clinical judgment faith bias whereby they may be less likely to make judgments reflecting a faith bias. This faith competence might come from a broad conceptualization of multicultural competence, if these clinicians view religiousness and spirituality as important and salient parts of the cultural backgrounds of their clients. On the other hand, their racial competence may have a collateral effect on their ability to deal with religious and spiritual issues and translate to a similar faith competence; their racial competence may generalize to a faith competence. Either way, if faith competence is part of a broader multicultural competence, then these clinicians may be less likely than others to allow stereotyping, salience bias, and the representativeness heuristic to interfere with their clinical decision-making. Hence, they may be less prone than other clinicians to succumb to clinical judgment faith bias.

On the other hand, some writers contend faith competence and multicultural competence are separate and distinct competencies (e.g., Richards \& Bergin, 2000; Stanard, Sandhu, \& Painter, 2000). Counselors - even multicultural ones - typically receive little training in addressing religious and spiritual issues (Shafranske \& Malony, 1990). If faith and multicultural competence are separate competencies, then multiculturally competent counselors may not be any less prone to clinical judgment faith bias than non-multiculturally competent counselors. This idea has yet to be empirically tested.

Multicultural competence is often conceptualized as a collection of competencies rather than a single competency. Sue, Arredondo, and McDavis's (1992) seminal article 
on multicultural counseling competencies and standards proposed that training programs target clinicians' beliefs and attitudes, knowledge, and skills as three broad dimensions to be addressed in multicultural training, while the Professional Standards Committee of the Association for Multicultural Counseling and Development proposed 31 multicultural counseling competencies (Sue et al., 1992). The Multicultural Counseling Knowledge and Awareness Scale, one of the most widely-used measures of multicultural competence, conceptualizes multicultural competence as being comprised of two competencies: awareness of multicultural issues and a clinician's knowledge of how to address multicultural issues which arise (Ponterotto \& Potere, 2003). Examining how each of these multicultural competencies influences clinical judgment faith bias is warranted. Therefore, multicultural competence will be henceforth conceptualized as multicultural awareness and multicultural knowledge.

Clinician magnitude of personal faith. Similarly, a clinician's magnitude of personal faith may also have a moderating effect on any clinical judgment faith bias that exists. Most clinicians believe religion has more impact on the presenting problems of religious clients than on the presenting concerns of nonreligious clients (Lewis \& Lewis, 1985). Individual clinicians, however, differ greatly in their willingness to integrate religious issues into counseling (Frame, 2003). Most clinicians are willing to discuss faith with their clients if their clients bring up the issue first (Worthington et al., 1996), but the willingness of clinicians to integrate faith issues into counseling is strongly influenced by their religious beliefs. Religious clinicians are much more likely to view faith as relevant and important to the therapeutic process, and are more likely to bring it up in counseling than nonreligious clinicians (Kahle, 1998; O’Malley, Gearhart, \& Becker, 1984). 
Ostensibly, religious clinicians are more familiar with religious issues, at least those of their religion, than are nonreligious clinicians. Thus, religious clinicians are more likely to be familiar with how those issues impact their clients, including perhaps the relevant base rates of psychopathology among religious persons, and possibly also the research literature demonstrating faith bias in clinical judgment. At the very least, clinicians who are religious are likely to be aware of the issues surrounding the stereotype erroneously associating religiousness with psychopathology. If this is the case, then clinicians who are religious or spiritual may be less likely to allow stereotyping, salience bias, and the representativeness heuristic to interfere with their clinical decision-making. These clinicians may be more likely than other clinicians to have faith competence, and as such they may be less prone than other counselors to clinical judgment faith bias. Again, as religiousness and spirituality are distinct constructs which may exert different influences on clinical judgments, clinician religiousness and spirituality should be measured separately.

Clinician view of faith helpfulness. A third clinician characteristic which may be associated with resilience to clinical judgment faith bias is a clinician's view of faith helpfulness, or that clinician's professional beliefs towards religious and spiritual matters in counseling. Three distinct aspects of a clinician's worldview include personal beliefs about how the world works, personal religious practices, and professional attitude towards faith in counseling (Beutler \& Bergan, 1991; Hecker et al., 1995; Worthington et al., 1996). Hillowe (1985) found that the personal religious practices of clinicians did not impact their clinical judgments, while their personal attitudes about religion did. While therapists' degree of involvement with a religious organization had no significant impact 
on their judgments of clients, their personal religious attitudes were significantly related to their professional ratings of the number of sessions required for religious clients to make notable progress in therapy (Hillowe, 1985). Clinicians' professional attitudes about mental health and pathology are greatly affected by their own personal values, and in therapy, clinicians' values appear to hold more influence than their clients' values. For example, Hecker, Trepper, Wetchler, and Fontaine (1995) observed "that when client and therapist values converge, the therapist is likely to rate the client as improving, despite the fact that clients may rate themselves as not having improved, or a standardized measure of symptom alleviation does not indicate improvement (Beutler et al., 1983)" (cited in Hecker et al., 1995, pp. 261-262). In other words, the personal attitudes and values of clinicians impact the professional process and outcome of their clinical work, even when clinicians do not intend this to occur (Beutler \& Bergan, 1991). A clinician may have agnostic or atheist personal beliefs and no particular religious practices, yet still hold respect for clients who have strong religious beliefs and think those beliefs have a direct impact on the process of therapeutic change which takes place for those clients (Lewis \& Lewis, 1985). Thus clinicians who view faith as being helpful and relevant to the counseling process may, too, have faith competence and be less likely to allow stereotyping, salience bias, and the representativeness heuristic to interfere with their clinical decision-making. For the same reasons as religious and spiritual clinicians, those who view faith as helpful and relevant to counseling may be less prone than other counselors to clinical judgment faith bias, even if they do not consider themselves to be religious or spiritual. In fact, several studies suggest religious clinicians often feel a sense of internal conflict over the integration of their personal religious and professional beliefs 
which is not typically present in nonreligious clinicians (Eckhardt, Kassinove, \& Edwards, 1992; Kassinove \& Uecke, 1991). Because personal religious beliefs and professional beliefs about the helpfulness of religion to counseling are distinct aspects of clinical values, each bears separate empirical examination.

\section{Purpose}

The primary purpose of this dissertation is to examine the hypothesized phenomenon of clinical judgment faith bias and which clinician attributes are associated with resilience to this bias. The term Clinical Judgment Faith Bias refers to a hypothesized tendency for clinicians to make more pathological clinical judgments for clients with higher magnitudes of faith than for otherwise identical clients with socially normative faith. The primary question of interest is whether clinical judgment faith bias occurs and, if so, under what circumstances (i.e., with which clinician attributes). More specifically, this dissertation tests whether clinician multicultural awareness, multicultural knowledge, personal religiousness, personal spirituality, or view of faith helpfulness are associated with resilience to this bias.

\section{Hypotheses}

Faith bias hypotheses. Three hypotheses are proposed to explain clinical judgment faith bias. These hypotheses are termed the Faith Type hypothesis, the Faith Magnitude hypothesis, and the Faith Combination hypothesis.

The Faith Type hypothesis postulates that clinicians view religious clients as more pathological than spiritual clients. Based on the aforementioned research on clinical judgment and faith, clinicians may be prone to clinical judgments which are biased by clients' religious beliefs, thus making more pathological clinical judgments for religious 
clients than for other clients. The presence of religious beliefs alone may be enough to activate salience bias and the representativeness heuristic for some clinicians, leading them to invoke stereotypes of religious people and make biased judgments.

Faith Type hypothesis: When making clinical judgments of clients presenting with anxiety symptoms, clinicians are predicted to make more pathological diagnostic and prognostic judgments for religious cases than for otherwise identical spiritual cases.

The Faith Magnitude hypothesis proposes that when making clinical judgments, clinicians tend to respond to the magnitude of client faith (both religious and spiritual) rather than the mere presence or absence of client religiousness or spirituality. In other words, as the magnitude of faith increases from low to moderate to high, faith bias is predicted to increase. Faith bias is predicted to be strongest for highly religious and spiritual clients, less strong for moderately religious and spiritual clients, and weakest for clients with low religiousness and spirituality. According to behavior decision-making theory, using the representativeness heuristic as a cognitive shortcut may lead clinicians to automatically access stereotypes of religious people and make unconscious judgments based on these stereotypes, without considering the base rates of no higher rates of psychopathology in this population. Salience bias brings these stereotypes to mind as the magnitude of faith increases, and clinicians become increasingly likely to show salience bias - and use the representativeness heuristic as a cognitive shortcut. The greater the magnitude of client faith, the more likely judgment bias is to occur.

Faith Magnitude hypothesis: When making clinical judgments of clients presenting with anxiety symptoms, clinicians are predicted to make increasingly 
more pathological diagnostic and prognostic judgments for cases as the magnitude of religiousness or spirituality portrayed in the cases increases from low to moderate to high.

The Faith Combination hypothesis anticipates that the type of faith clients manifest interacts with the magnitude of this faith such that the increase in faith bias as magnitude increases is more pronounced in religious clients than in spiritual clients. This hypothesis predicts a synergistic interaction in which faith type enhances the effect of faith magnitude on clinical judgments. As the magnitude of client faith increases, faith (especially religiousness) becomes more salient, salience bias becomes more likely, and clinicians become increasingly likely to use the representativeness heuristic to access stereotypes of religious people. Religious faith is predicted to result in faith bias at lower magnitudes than spiritual faith does. In other words, client magnitude of faith (low, medium, or high) is predicted to interact with client type of faith (religious or spiritual). This interaction is illustrated in Figure 1.

Faith Combination hypothesis: When making clinical judgments of clients presenting with anxiety symptoms, client magnitude of faith is predicted to interact with client type of faith such that the increase in pathology judgments as magnitude increases is more pronounced for religious cases than for spiritual cases.

Resilience hypothesis. Several clinician attributes may be associated with resilience to clinical judgment faith bias - specifically, multicultural awareness, multicultural knowledge, personal religiousness, personal spirituality, and view of faith helpfulness. The Resilience hypothesis postulates that these attributes may confer 


\section{Figure 1}

Pathology ratings of religious and spiritual cases at increasing magnitudes of client faith

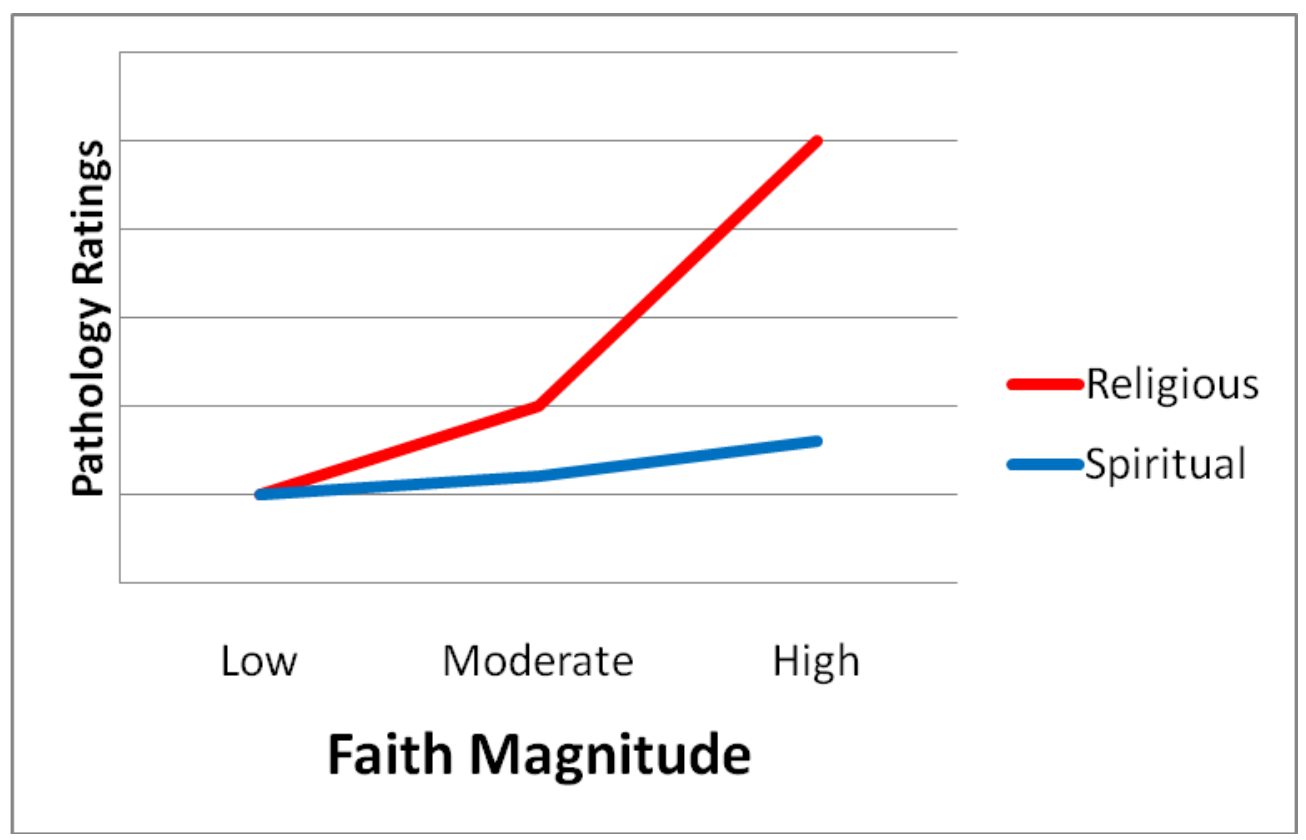

resilience to clinical judgment faith bias, moderating the impact of client faith magnitude, client faith type, or their interaction on clinical judgments. Clinicians who are high in these characteristics are anticipated to be less prone to making clinical judgments biased by client faith than clinicians who are low in these characteristics. In other words, each one of these clinician attributes is predicted to moderate the effect of client faith magnitude, client faith type, and their interaction on clinical judgments, thereby reducing faith bias in clinicians who are high in any one of these characteristics. The 2-way interactions of each individual clinician attribute with faith type and with faith magnitude, and the 3-way interactions of each clinician attribute with the 2-way interaction of faith type and magnitude, are predicted to buffer or weaken the impact of faith type, faith magnitude, and their 2-way interaction on clinical judgments. These 2- 
way and 3-way interactions comprise the Resilience hypothesis. A prototypal depiction of the Resilience hypothesis for any one of these clinician attributes is illustrated in Figure 2. For the sake of illustration, each attribute has been trichotomized into only three levels for clarity, though the attributes are continuous in nature.

Resilience hypothesis: When making clinical judgments of clients presenting with anxiety symptoms, the clinician attributes of (1) multicultural awareness, (2) multicultural knowledge, (3) personal religiousness, (4) personal spirituality, and (5) view of faith helpfulness are predicted to moderate the impact of (a) client faith type, (b) client faith magnitude, and (c) their interaction on clinical judgments, such that client type and magnitude of faith and their interaction act to bias the judgments of all clinicians, but this interaction is weaker in clinicians who are high in these attributes and stronger in clinicians who are low in them.

\section{Summary}

This study endeavors to test the aforementioned four hypotheses, for the purpose of explicating the complex and multidimensional phenomena of a potential clinical judgment faith bias. It is anticipated this project will have a small but noteworthy contribution to the field of the psychology of religion. Clinical judgment faith bias is a new term, and it refers to a concept on which very little past research has been conducted. Past research has demonstrated clinicians do sometimes make biased judgments based on client religion, but the research is mixed about what those specific cases are. It appears, though, at least in some cases, clinicians may be inadvertently providing less than optimal care for some clients - even misdiagnosing people. Thus it is important to better understand this specific type of clinical judgment bias in order to provide more effective 


\section{Figure 2}

Pathology ratings of religious and spiritual cases at increasing magnitudes of client faith by clinicians with low, moderate, and high levels of any one clinician attribute

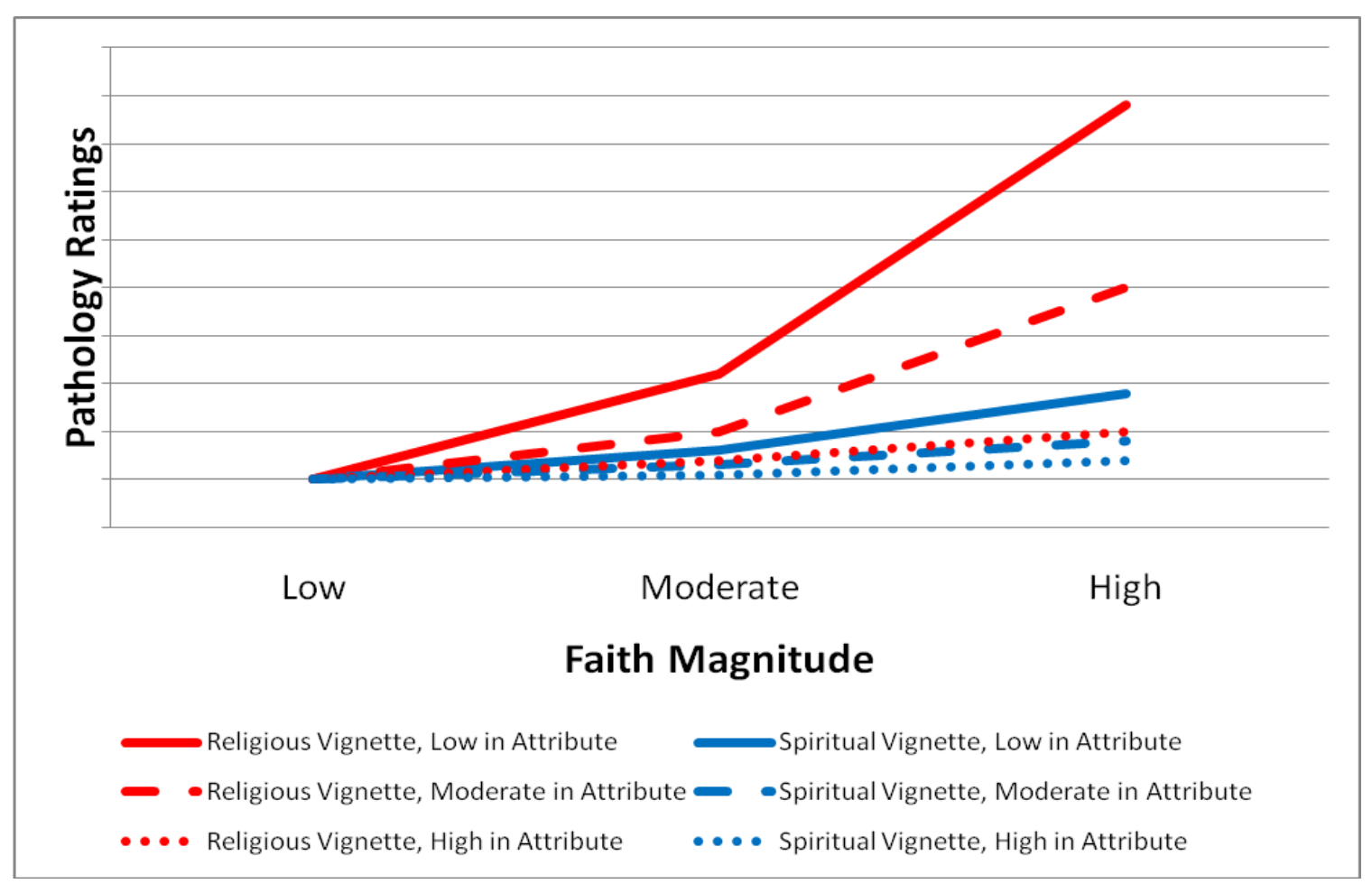

and multiculturally competent clinical care. The effect on clinical judgment of client faith, clinician faith, clinician multicultural competence, and clinician view of faith helpfulness have all been researched prior to this study, but no previous research has combined all of these variables into a single study. Furthermore, the religion and spirituality vignettes used in past clinical judgment research were created using much less rigorous techniques than the vignettes used in the current study, which were developed so that their magnitude of faith could be precisely measured and experimentally manipulated. Thus the results of this study - and the vignettes themselves - may be of 
interest to researchers in the fields of clinical judgment, the psychology of religion, and multicultural counseling alike. 


\section{Chapter 2}

\section{Literature Review}

Values are an important component of psychotherapy. Though many clinicians strive to keep their values and beliefs from being known to their clients, few if any therapists believe therapy is value free (Worthington, 1986). Psychotherapy has its own set of values which inform how mental health is conceptualized, how mental illness is treated, and how positive therapeutic change should occur during the course of counseling. For example, beneficence, nonmaleficence, confidentiality, respect for diversity, and opposition to suicide and abuse are a few of psychotherapy's values (c.f., APA, 2002; Koocher \& Keith-Spiegel, 1998). Clinicians, too, bring their own values and beliefs with them to psychotherapy, though ideally they set those values aside, acknowledging them and using them when appropriate, but not letting those values interfere with providing effective therapeutic treatment (Spengler, Strohmer, Dixon, \& Shivy, 1995). Clients bring their own values and beliefs with them into therapy as well. Unlike clinicians, though, clients do not set aside their values at the door. In fact, most clients expect their counselors to be sensitive to and respectful of their values, and most clinicians do treat their clients' values with both sensitivity and respect (Belaire \& 
Young, 2002). On the other hand, considerable research indicates in successful therapy, clients often change their values to be more in line with those of their counselors (Worthington, Kurusu, McCullough, \& Sandage, 1996). Clinicians must be mindful of the role values play in counseling to ensure sound clinical judgments.

\section{Religious and Spiritual Values in Clinical Work}

Spiritual and religious values constitute a special set of values which often arise in counseling: faith values. Of the many value issues which come into play in counseling (e.g., treatment values, mental health values, work values, moral values, conservative and liberal values, conventional and unconventional values) few are as important to their value-holders, or as contentious, as spiritual and religious values. Frequently, clients come to counseling with a specific religious or spiritual problem which directly or indirectly impacts the course of counseling. According to Shafranske and Malony (1990), as many as one in six clients may come to counseling with a presenting issue involving religion or spirituality. Quackenbos, Privette, and Kleinz (1985) reported $81 \%$ of people surveyed believe religious and spiritual values are important and need to be adequately addressed in the therapeutic process. Drawing from a questionnaire given to 79 psychiatric inpatients, D’Souza (2002) noted $82 \%$ of inpatients believe it is important for therapists to be aware of their spiritual beliefs, while $69 \%$ believe the spiritual needs of clients should be considered by the therapist in treating mental illness. Studies examining different populations have yielded similar results. For example, Lindgren and Coursey (1995) found $67 \%$ of clients desire greater opportunities to discuss spirituality in the course of treatment. Martinez (1991) noted a majority of parents want counselors to incorporate religious and spiritual themes into the counseling of their children. In a study 
examining clients' willingness to include aspects of spirituality into treatment, Rose, Westefeld, and Ansely (2001) found 55\% of clients want to discuss religious or spiritual issues in counseling and believe it is appropriate to do so. Thus, a surprising number of clients bring spiritual or religious issues with them into counseling.

Even when spiritual issues are not the focus of therapy, religion is an element of cultural diversity often encountered in clinical practice. Religions claim $87 \%$ of the world's population in their membership, and up to $95 \%$ of the world's population believes in either God or a higher power of some kind (Frame, 2003). More than 257 million people (85.2\%) in North America count themselves as Christians, while another 45 million people (14.8\%) are members of other religions, including Judaism (5.9 million), Islam (4.1 million), Buddhism (2.1 million), and Hinduism (1.1 million) (Richards \& Bergin, 2000). Gallup polls indicate two thirds of Americans rate religion as being important or very important to them (Bergin, 1991). Kahle and Robbins (2004) noted $95 \%$ of Americans believe in some sort of God, while $80 \%$ believe in a personal God, $90 \%$ engage in prayer, and $75 \%$ pray on a daily basis (Gallup, 1999, 2001). A full $92 \%$ of Americans state a religious preference (Gallup, 2001), while $66 \%$ are actually members of churches or synagogues (Gallup News Service, 2002) and 90\% desire some kind of religious education for their children (Hoge, 1996). Similarly, 86\% of Americans affirm religion is very or fairly important in life, $63 \%$ believe religion can answer all or most of today's problems, and $76 \%$ believe the Bible is the actual or inspired word of God (Gallup News Service, 2001, 2002). Also, Frame (2003) pointed out in the United States, immigration has shifted the demographics of the country, with the number of Spanish-speaking Catholics and Middle-Eastern Muslims increasing dramatically since 
the 1960's. As a result, therapists are increasingly being called upon to have competence in addressing religious and spiritual issues in counseling, or at the very least to be aware of how these issues impact the lives of their clients and their clinical judgments.

A growing number of clinicians believe it is appropriate to include spirituality, religion, or both in counseling, at least under certain circumstances. According to Bergin (1991), $68 \%$ of surveyed mental health professionals agree spirituality is part of a mentally healthy lifestyle, and $41 \%$ indicate this is important in guiding therapy. Worthington et al. (1996) pointed out while a few clinicians are committed to including formal religion in counseling and even fewer are opposed to discussing formal religious issues in counseling, most are accepting of religion if it comes up in counseling while not pushing for its inclusion. Several studies have shown the majority of clinical psychologists (Gerson, Allen, Gold, \& Kose, 2000), licensed professional counselors (Hickson, Housley, \& Wages, 2000), psychotherapists (Kahle, 1998), marriage and family therapists (Carlson, Kirkpatrick, Hecker, \& Killmer, 2002), and social workers (Gilbert, 2000) support the integration of spirituality and religion into therapy. In one of the largest surveys of its kind, Shafranske and Malony (1990) surveyed more than four hundred psychologists and found $74 \%$ believe religious and spiritual issues are within the scope of psychotherapy, $87 \%$ believe it is appropriate for psychologists to know the religious background of their clients, and $59 \%$ believe it is appropriate to use religious concepts in therapy. Similarly, $73 \%$ of licensed professional counselors believe spirituality is a universal phenomenon which is "important" or "vitally important" in the counseling process (Hickson et al., 2000). Thus, psychotherapists are willing to integrate spiritual and religious issues into psychotherapy, though they often prefer clients to bring 
it up, and they may be more likely to use spiritual rather than religious terminology when broaching the topic themselves (Kahle, 1998).

While most therapists are willing to include religious or spiritual themes into the therapeutic process (e.g., Bergin, 1991; Richards \& Potts, 1995), individuals who identify as being religious or spiritual often believe most counselors are secular and will ignore, minimize, or even pathologize their religious beliefs (Collins, 1988; Houts \& Graham, 1986; Worthington; 1986). Often, religious people prefer religious or pastoral counseling, or they seek out therapists with similar values (Bergin, 1991; McLatchie \& Draguns, 1984). Some evidence suggests very religious people only use professional therapy as a last resort, after trying everything else (Sell \& Goldsmith, 1988; Veroff, Kulka, \& Douvan, 1981). Richards and Bergin (2000) speculated religious individuals significantly underutilize traditional mental health services - especially very religious persons. Sometimes, individuals in need of services might avoid counseling altogether (Guinee \& Tracey, 1997; McLatchie \& Draguns, 1984; Morrow, Worthington, \& McCullough, 1993; Rose et al., 2001; Worthington, 1986). Thus there is a public perception that clinicians are biased against religion.

This public perception is not without merit. In spite of most therapists' willingness to discuss spiritual and religious issues in counseling, psychotherapy is a secular humanistic field, and psychology as a profession has historically shown bias against religion (Houts \& Graham, 1986). Frame (2003) listed several reasons why psychology as a field has often neglected religion. First, the relationship between psychology and religion has long been tenuous, characterized by conflict and mutual rejection (Blass, 2001; Frame, 2003; Richards \& Bergin, 2000). Religious leaders have 
often opposed scientific thinking, viewing it as conflicting with religious teachings, and many psychologists have held equally oppositional views of religion, viewing it as irrelevant, irrational, or even a symptom of neurosis or psychosis (Boadella, 1998; Cornett, 1998; Khalili, Murken, Reich, Ali Shah, \& Vahabzadeh, 2002). Second, the epistemological assumptions of psychology conflict with those of religion. Religion is based on faith, tradition, and the teachings of authority, while psychology is based on empiricism and the scientific method (Cornett, 1998; Frame, 2003). Third, psychologists have often associated religion with psychopathology. Though there is little empirical evidence to support this notion, psychoanalysts, behaviorists, and cognitive psychologists alike have favored the idea that religion is irrational, the sign of an uneducated mind, or even a symptom of mental illness (Cornett, 1998; Frame, 2003). Fourth, clinicians do not receive training in how to address these issues in the way pastors, priests, seminarians, and other religious professionals do. The clinical education of psychologists typically lacks training for religious and spiritual issues. For example, fewer than $25 \%$ of counselor education programs address religion or spirituality in their curricula, so mental health professionals often are not trained to address religion in therapy, even if they are inclined to do so (Frame, 2003). Fifth, psychologists commonly believe only clergy should address religious issues. Since clergy have been trained specifically to address people's religious concerns, many psychologists simply believe religion is not in their job description (Frame, 2003). For this reason, only $29 \%$ of psychologists believe they should bring up the topic of religion in counseling (Bergin, 1991), in spite of the fact perhaps more than $80 \%$ of clients feel religion is relevant and important to address in counseling (Frame, 2003; Quackenbos, Privette, \& Kleinz, 1985), and in spite of the 
willingness of most psychologists to discuss religious or spiritual matters should their clients initiate these discussions.

Finally and perhaps most importantly, psychologists tend to be less religious than the general population and even less religious than other professionals (Bergin, 1991) - a difference Bergin (1980) termed a religiousness gap between psychologists and others. Consequently, psychologists are more likely to see spiritual and religious issues as deviant, pathological, or simply irrelevant since these issues are often immaterial to their personal lives, whereas to clients spiritual and religious issues may be an important part of their belief systems, support networks, or cultural backgrounds. This increases the chances clinicians will ignore, discount, or pathologize religious issues, even when they are relevant to clients' presenting concerns. Furthermore, behavioral decision-making theory and social cognition research suggest clinicians who fail to examine their own values and preconceived notions are more likely to make biased clinical judgments (e.g., Kahneman \& Tversky, 1973; Nisbett \& Ross, 1980; Salovey \& Turk, 1986; Spengler et al., 1995), and because clinicians seldom receive any training in addressing religious and spiritual issues, the religiousness gap and its implications for clinical treatment are likely to go unnoticed and unexamined. Thus the public perception that psychologists are biased against religious issues may not be baseless; the religiousness gap between psychologists and the general population increases the potential for clinicians to make biased clinical judgments, whereby they unduly pathologize individuals who express religious or spiritual beliefs. 


\section{Clinical Judgment Faith Bias}

There is tentative support in the research literature for the contention that clinicians show faith bias in some of their clinical judgments. The mental health literature has shown the base rates of psychopathology are not significantly higher among religious people than they are among nonreligious people (Miller \& Kelley, 2005). There are some exceptions to this finding. Generally, though, the literature supports the notion that religion is not associated with increased risk for psychopathology. In fact, much of the literature suggests just the opposite, in that religiousness and psychopathology are negatively correlated (Koenig, 1998). Nevertheless, practicing clinicians sometimes evaluate religious clients more pathologically than otherwise identical secular or nonreligious clients. This suggests faith bias may exist in the clinical decision-making of practitioners.

Client religiousness sometimes biases clinicians' judgments of pathology. Sanderson, Vandenberg, and Paese (1999) found when 67 mental health professionals were asked to rate 18 case vignettes on their degree of mental health, clinicians made judgments based not on whether the vignettes had religious content, but on the degree this religious content deviated from the norm. The more a case's religious content deviated from the norm, the less mentally healthy clinicians judged that case to be. In a particularly well-done study by O'Connor and Vandenberg (2005), 110 mental health professionals gave higher ratings of pathology to members of less mainstream religions than to members of more mainstream religions. They made significantly higher pathology ratings for Nation of Islam cases than for Latter Day Saint (Mormon) cases, and significantly higher pathology ratings for Latter Day Saint cases than for Catholic cases. 
Houts and Graham (1986) found 48 clinicians rated moderately religious clients as having greater psychopathology than either very religious or nonreligious clients. Finally, studies by Gartner and colleagues (1990a, 1990b) found 363 clinical psychologists did not give different severity ratings on the Clinical Judgment Scale to members of different religious groups (fundamentalist Christians, evangelical Christians, or Mormons). Clinicians, however, did rate clients with extreme ideologies (i.e., members of very conservative or very liberal groups, either religious or political) as having more severe psychopathology, more stress, less empathy, and less maturity than other clients.

There is also some evidence to suggest clinicians' diagnostic judgments may sometimes be biased by client religiousness, at least when clinicians are diagnosing anxiety disorders. The aforementioned studies by Gartner and colleagues (1990a, 1990b) found when clinicians were asked to give diagnoses for nearly equivalent cases, clients with extreme ideologies were almost three times more likely to be diagnosed with Obsessive Compulsive Disorder than clients with less extreme ideologies, but they were less likely to be diagnosed with Generalized Anxiety Disorder. This is particularly surprising, given the prevalence of OCD in the general population $(2.5 \%)$ is half that of GAD (5\%), according to the DSM-IV-TR (Diagnostic and Statistical Manual of Mental Disorders - Fourth Edition, Text Revision; American Psychiatric Association, 2000). Given OCD is more difficult to treat and viewed as more disabling than GAD (Gartner et al., 1990a), this could indicate a specific diagnostic faith bias with anxiety disorders.

Suspecting such a bias, Yossifova and Loewenthal (1999) replicated the Gartner et al. (1990a, 1990b) studies in Great Britain, using a within-participants design to increase sensitivity, surveying lay people instead of psychologists, and manipulating only 
non-political religious activity. They found when 96 people were shown a case with symptoms of OCD, they were more likely to judge the case as having obsessional symptoms when it described a highly religious person than when it described a person low in religiousness. Taken together, these two studies suggest the possibility of a stereotype associating religious behaviors and obsessional symptoms, influencing the perceptions of lay persons and clinicians alike.

Clinicians' prognostic judgments are also sometimes biased by client religiousness. An often-cited clinical analogue study by Hillowe (1985) surveyed 115 doctoral level psychologists currently engaged in the practice of psychotherapy, giving them three measures of clinician religiousness and two pairs of clinical vignettes and asking them to rate the prognoses of the clients portrayed in the vignettes. One pair of vignettes described a client with the DSM-III (Diagnostic and Statistical Manual of Mental Disorders, $3^{\text {rd }}$ edition; American Psychiatric Association, 1980) diagnoses of Obsessive Compulsive Neurosis and Avoidant Personality Disorder, and another pair described a client with Major Depression, Single Episode and Dependent Personality Disorder. In each pair, one vignette described a religious client and one described a nonreligious client. Hillowe (1985) found the psychologists perceived religious clients as being less appropriate for counseling than nonreligious clients. Appropriateness for counseling is a construct which is similar to (and directly related to) client prognosis. Likewise, Jones (1991) gave 59 licensed Kentucky psychologists three cases depicting clients who were nonreligious, very religious, or religiously doubtful (i.e., questioning traditional religious beliefs and rejecting some of them, but not rejecting all traditional religious beliefs or rejecting religion completely, similar to Batson and Ventis's (1982) 
quest dimension of religiousness). She found nonreligious counselors rated the religious cases as having the worst prognoses. These studies suggest that, overall, client religiousness may sometimes bias clinicians' prognostic evaluations, as clinicians in these studies showed a tendency to give religious clients more pathological prognoses than secular clients with identical symptoms.

\section{Religion and Psychopathology}

Viewing religion as being psychopathological is not entirely without merit. Though religious involvement has been empirically shown to be positively correlated with both physical health (Oman \& Thoresen, 2005) and mental health (Miller \& Kelley, 2005), there are some exceptions to this finding. Literature reviews on religion and mental health (e.g., Davis, Kerr, \& Kurpius, 2003; Harris, Schoneman, \& Carrera, 2002; Koenig, Ford, George, Blazer, \& Meador, 1993; Koenig, George, Blazer, Pritchett, \& Meador, 1993; Pfeifer \& Waelty, 1999) have found a small positive correlation between anxiety and religion, suggesting people with anxiety disorders are more religious than other people, though there is little evidence to support the converse - religious people are not necessarily more susceptible to anxiety. Religious struggle has been shown to be associated with elevated mortality rates (Oman \& Thoresen, 2005). Furthermore, in an extensive and well-done literature review of the literature on religion, mental health, and psychopathology, Miller and Kelley (2005) noted for every mental disorder they reviewed, "there are at least a few studies that display a positive correlation between religious belief or activity and pathological symptoms" (p. 464). As these authors noted, "in teasing apart the multiplicity of influences on mental health, it quickly becomes clear that a consistent, robust, and unidirectional relationship between mental health and 
religiousness or spirituality is an illusion - the reality is far more subtle and complex" (Miller \& Kelley, 2005, p. 462).

In some cases, the mixed and conflicting results which have been found in the research on religion and psychopathology are due to different ways in which religiousness has been measured, or different aspects of religion which are examined. For example, religiousness is frequently bifurcated in the literature into extrinsic and intrinsic forms. Extrinsic religiousness refers to means-oriented, externally motivated religious behavior performed as a means to a secular end such as gaining social approval or ego reinforcement. Intrinsic religiousness refers to ends-oriented, internally motivated religious behavior performed for its own sake or because of a heartfelt belief it is the right thing to do (Allport \& Ross, 1967). Extrinsic religiousness has been consistently found to be positively correlated with psychopathology (Bergin, 1991), depression (Watson, Morris, \& Hood, 1988a, 1988b, 1988c), trait anxiety (Baker \& Gorsuch, 1982), prejudice (Donahue, 1985), dogmatism, authoritarianism, irresponsibility (Kahoe, 1974), psychological distress, less effective coping strategies, and socially inappropriate behavior (Miller \& Kelley, 2005). On the other hand, intrinsic religiousness has been negatively correlated with these traits and positively associated with empathy, internal self-awareness, and self-consciousness (Watson et al., 1988a, 1988b; Watson, Hood, Morris, \& Hall, 1987).

Overall, however, there is no evidence to suggest religiousness is associated with psychopathology. In fact, most forms of religious belief and expression are negatively associated with psychopathology and positively associated with mental (and physical) health. Miller and Kelley (2005) noted empirical research and clinical experience 
overwhelmingly suggests religiousness positively influences mental health, translating into not merely the absence of psychopathology but also increased happiness and life satisfaction, facilitated construction of meaning and life goals, longer life-span, and even higher income and educational attainment. Religious involvement is also associated with increased social support and greater happiness (Argyle, 1999). In a logistic regression analysis of seven dimensions of religiousness and their relationship to lifetime psychiatric and substance use disorders, all seven dimensions were found to be associated with reduced risk for internalizing disorders, externalizing disorders, or both (Kendler, Liu, Gardner, McCullough, Larson, \& Prescott, 2003). Several social psychological studies have indicated religiousness is negatively correlated with drug abuse, alcohol use, sexual permissiveness, teenage pregnancy, and some delinquent acts and is positively correlated with self-esteem, family cohesion, and self-perceived well-being (Bergin, 1991). Intrinsic religiousness (in contrast to extrinsic religiousness) has been correlated with greater wellbeing, more appropriate social behavior, and more effective coping methods (Batson, Schoenrade, \& Ventis, 1993). Moreover, an extensive meta-analysis by Smith, McCullough, and Poll (2003) encompassing 147 studies and nearly 99,000 participants found a significant negative correlation between religious involvement and symptoms of depression. Likewise, similar but smaller reviews of the empirical literature have demonstrated no differences in the rates of anxiety, schizophrenia, other psychotic disorders, or obsessive-compulsive disorder among religious versus nonreligious people (Miller \& Kelley, 2005). Thus religious persons are not more likely to exhibit symptoms of psychopathology than nonreligious persons. While religion often plays a role in the 
expression of symptoms (e.g., a psychotic delusion of being Jesus Christ) and in coping with these disorders (e.g., prayer), there is no evidence it is etiologically causative.

The relationship between religion and psychopathology found in the research literature also depends upon which aspects of religiousness and psychopathology are being investigated. Gartner (1996) pointed out the research suggesting an association between religiousness and psychopathology has focused on authoritarianism, dogmatism, tolerance of ambiguity, rigidity, suggestibility, self-actualization, and temporal lobe epilepsy, while the research supporting an association between religiousness and mental health has focused on physical health, mortality, suicide, drug use, alcohol abuse, delinquency and criminal behavior, divorce and marital satisfaction, well-being, outcome, and depression. Meanwhile, research studies focusing on overall health, anxiety, psychosis, self-esteem, sexual disorders, intelligence, education, and prejudice have shown ambiguous or complex associations between religion and mental health (Gartner, 1996). The specific mental health phenomenon or diagnosis being investigated, then, appears to make a notable difference in the presence or absence, degree, and directionality of the relationship between mental health and religiousness.

\section{Clinical Judgment Faith Bias and Faith Magnitude}

The studies pointing to the existence of a clinical judgment faith bias also suggest such a bias may only emerge in response to clients with high magnitudes of religiousness - clients with socially deviant religious beliefs and practices. Deviance is a term used to refer to attitudes and behaviors used by certain people in certain situations which violate shared normative expectations, or socially-constructed notions on what kinds of behaviors and attributes are appropriate and inappropriate or typical in different situations 
(Kaplan \& Johnson, 2001). Four relevant types of deviance exist: statistical deviance, comparative deviance, pathological deviance, and social deviance (Barlow \& Durand, 1995).

Statistical deviance simply refers to phenomena which are numerically unusual. These phenomena are considered to be deviant merely because they seldom occur; they are found towards the outer edge of the normal curve (Krathwohl, 1998). Religiousness itself is not statistically deviant. As mentioned previously, the majority of the world's population is religious, with $87 \%$ of people belonging to a religion and up to $95 \%$ of people believing in a higher power of some kind (Frame, 2003), so nonreligious people are in many respects a social group which could be considered to be statistically deviant. People vary widely, however, in their degree or magnitude of religiousness, so some forms of religiousness (e.g., extreme fundamentalist groups) are numerically unusual. Like many psychological traits, though, religiousness approximates a normal curve, with the majority of the people in the middle of the distribution and much fewer numbers of people falling at either end. Statistically speaking, then, moderate religiousness is the norm; the majority of people across the world are moderately religious (Momen, 1999).

On the other hand, people with either very low or very high levels of religiousness fall on the extreme ends of the normal curve, deviating from the statistical norm.

In contrast to statistical deviance, comparative deviance refers to phenomena which are unusual or deviant when compared to "the norm." "The norm" varies widely depending on the cultural context and the phenomena of interest, but usually refers to the characteristics of the group doing the comparing. In the movie Star Trek: Generations, actor Malcolm MacDowell opined somewhat famously that normal is "what everyone 
else is and you are not" (Berman, Lauritson, Williams, \& Carson, 1994). Comparative deviance refers to the converse of this: what you are and everyone else is not. In terms of religiousness, the comparative deviance of a group's religiousness depends on the religious beliefs and behaviors of the group doing the comparing. To a religious group, a nonreligious person is comparatively deviant, while to a moderately religious group, both highly religious and nonreligious people are comparatively deviant. Psychologists tend to be significantly less religious than the general population (Bergin, 1991), so from a psychologist's perspective, both highly religious and moderately religious people appear to be comparatively deviant, if the psychologist uses other psychologists to represent the "normal" control group.

Another form of deviance - and the form of primary concern to psychologists - is pathological deviance. Pathological forms of deviance are not merely numerically infrequent or socially unacceptable, but are associated with pathological (i.e., dysfunctional) behavior (Barlow \& Durand, 1995). The Diagnostic and Statistical Manual of Mental Disorders - Fourth Edition, Text Revision (DSM-IV-TR) treats a wide variety of behaviors as symptoms of pathology, provided these behaviors represent "a clinically significant behavioral or psychological syndrome or pattern" in an individual which is related to significant distress or impairment of functioning, and provided these behaviors are not "merely an expectable and culturally sanctioned response to a particular event" (DSM-IV-TR, p. xxxi). The DSM-IV-TR cautions, though, "neither deviant behavior (e.g., political, religious, or sexual) nor conflicts that are primarily between the individual and society are mental disorders unless the deviance or conflict is a symptom of a dysfunction in the individual" (p. xxxi). O’Connor and Vandenberg (2005) also 
noted the DSM-IV contains an "articles of religious faith" exemption (p. 611): in order for a given belief to be a delusion, the DSM-IV maintains this belief must be "based on an incorrect inference about external reality that is firmly sustained despite what almost everyone else believes and despite what constitutes incontrovertible evidence to the contrary" - and, furthermore, this belief cannot be "one ordinarily accepted by other members of the individual's culture or subculture (e.g., it is not an article of religious faith)" (DSM-IV, p. 765).

As mentioned above, a misconception exists that religiousness is pathologically deviant - that religion is associated with psychopathology (Frame, 2003). This is a source of evident bias and a recognized stereotype. Many prominent figures in the history of psychology have described religiousness as being pathological, unhealthy, delusional, misguided, or the result of psychological defenses or the avoidance of personal responsibility, including Freud (1927), Ellis (1980), Skinner (1953), Yalom (1980), and many humanists (Frame, 2003). There is no evidence to suggest base rates of psychopathology are higher for religious people than nonreligious people - this is an unfounded stereotype (Miller \& Kelley, 2005). Yet clinicians do occasionally make more pathological clinical judgments, either more disabling diagnoses or more pathological prognoses, for religious clients. Since these clinical judgments disregard the relevant base rates of pathology among religious clients, they presumably represent an interrelated set of biases which should be addressed - biases which are referred to here collectively as clinical judgment faith bias.

On the other hand, social deviance refers to phenomena which do not merely differ numerically from the characteristics of the majority or from the features of a given 
comparison group, but to phenomena that violate perceived social or conventional norms (Newcomb \& Burns Loeb, 1999). From the perspective of social learning theory, a person's socially deviant beliefs and behaviors represent that person's failure to learn socially appropriate beliefs and behaviors. People tend to conform to social norms to the degree they feel close to others who endorse those norms. The more social ties a person has, the more invested that person is in the status quo and the less likely that person is to display deviant attitudes and behavior (Akers, 2000; Stark \& Bainbridge, 1996).

O'Connor and Vandenberg (2005) suggested clinical judgment faith bias may emerge, not merely in response to the presence of religious beliefs or behaviors, but in response to socially deviant forms of religiousness. Socially deviant forms of religiousness do not just differ from those of the majority or a given comparison group, but also violate social or conventional norms for what is considered to be appropriate or acceptable (Newcomb \& Burns Loeb, 1999). In the United States today, moderate religiousness is not only the statistical norm, but is socially acceptable as well. The U.S. is also a secular society, so nonreligious people are also generally accepted socially (except for the most radical forms of atheism). Only one form of statistically deviant religiousness is considered to be socially deviant - that is, high religiousness (Miller \& Kelley, 2005; Momen, 1999).

While clinicians may not consider religion itself to be pathological, O'Connor and Vandenberg (2005) proposed clinicians may nevertheless unduly consider socially deviant forms of religiousness to be more pathological than socially normative forms of religiousness. In their previously mentioned study, they observed clinicians made significantly higher ratings of pathology for a highly socially deviant religion than for a 
moderately socially deviant religion, and significantly higher ratings of pathology for the latter than for a mainstream or socially normative religion. As a result, O'Connor and Vandenberg (2005) were among the first researchers to provide evidence some clinicians may have a bias to pathologize socially deviant forms of religiousness.

\section{Moderate Religiousness}

Moderate religiousness is considered to be socially acceptable. Since, statistically, the majority of the world's population is moderately religious (Momen, 1999), moderate levels of religiousness are generally viewed as socially normative. Also, the United States is largely a secular society. The legal separation of church and state sets up a unique form of social approval for nonreligious people, so nonreligious people are generally accepted socially, except for the most radical or militant atheists (Momen, 1999). On the low end of the religiousness continuum, a person must be extremely nonreligious - even antireligious - in order to incur social judgments of inappropriateness. On the high end of the religiousness continuum, though, a religious person does not have to differ much from the moderately religious norm to be judged as socially deviant. Perhaps this is because of the amount of media attention devoted to religious extremists and religious fundamentalists, or a by-product of the stereotype of the crazy religious fanatic (Miller \& Kelley, 2005). Perhaps, too, this is a by-product of the secularization of American society (Momen, 1999). Whatever the reason, high religiousness is generally considered to be socially deviant in the United States today, whereas most forms of moderate and low religiousness are considered to be socially normative (Miller \& Kelley, 2005; Momen, 1999). 
In the United States, people who are moderately religious (and thus statistically normative) tend to hold moderately strong religious beliefs, attend religious gatherings occasionally, and agree with some (but not all) of their religion's doctrinal teachings (Abramowitz, Deacon, Woods, \& Tolin, 2004; Momen, 1999; Paloutzian \& Park, 2005; Richards \& Bergin, 2000). They are typically members of a major religious group usually Protestant or Catholic, and often one of the mainline Protestant denominations (Lutheran, Presbyterian, Episcopal/Anglican, or Methodist; McCullough, Weaver, Larson, \& Aay, 2000). By contrast, highly religious (and statistically deviant) people tend to hold very strong religious beliefs, attend religious gatherings frequently (more than once a week), and have high agreement with the doctrinal teachings of their religion (Abramowitz et al., 2004; Momen, 1999; Paloutzian \& Park, 2005; Richards \& Bergin, 2000). They are also typically members of a major religious group - often Protestant or Catholic, but they are more likely than moderately religious people to be members of more extreme fundamentalist or evangelical religious denominations (Thurston, 2000). On the other hand, people who are not religious (and who are statistically deviant towards the low end of the normal curve) tend to hold very weak or no religious beliefs, attend religious gatherings seldom or never, and disagree with religious teachings. They are not typically members of any religious group and may self-identify as Agnostic or Atheist (Abramowitz et al., 2004; Momen, 1999; Paloutzian \& Park, 2005; Richards \& Bergin, 2000).

There are also a number of non-mainstream religious groups in the United States which would be considered to be socially deviant by the majority of Americans. In some Native American and African-American communities, people believe spirits of the dead 
actively influence the daily lives of the living, and some people of African descent consult with the divine using cowrie shells (Boyer, 2001; Zea, Mason, \& Murguia, 2000). Some Pagan groups believe in the existence of sprites and fairies, and many Wiccans believe in magic (Berger, 1999; Berger, Leach, \& Shaffer, 2003). Some Latinos and Latinas believe they can communicate with the dead and apparitions (Shafranske, 2005). Hindus, Buddhists, and Sikhs often believe in reincarnation (Keller, 2000). Conservative Roman Catholics often believe priests' prayers literally turn wine into blood (Shafranske, 2000). Conservative Seventh-Day Adventists avoid movies, theaters, circuses, and night clubs, believing them to be immoral (Rayburn, 2000). Some fundamentalist Protestants believe men should not grow long hair or wear tight pants, and women should not cut their hair or wear jewelry, pants, short dresses, or makeup because doing so would reflect impurity (Thurston, 2000). Pentecostals often practice speaking in tongues and prohibit dancing, attending movies, and (in women) the wearing of makeup, jewelry, and short hair (Altemeyer \& Hunsberger, 2005; Dobbins, 2000). There are also a variety of religious cults that, by definition, are socially deviant belief systems (c.f., Momen, 1999), and whose members hold a variety of unusual, strange, "crazy," and sometimes illegal beliefs (Frame; 2003; Long \& Hadden, 1983). Each of these beliefs is perfectly acceptable among the group which holds it but is considered to be strange, abnormal, or even crazy among the majority of (often moderately religious) people in the United States today. Hence, these beliefs are considered to be socially deviant. Generally speaking, though, each of these groups is also considered by the majority of (moderately religious) Americans to be highly religious. Therefore, for the sake of simplicity, the current study focuses on highly religious people when it refers to "socially deviant" forms of 
religiousness and treats socially deviant religiousness and high religiousness as approximately equivalent terms because of their high collinearity.

\section{Religiousness, Faith Magnitude, and Psychopathology}

High faith magnitude is often believed to be associated with psychopathology because high magnitudes of religiousness are perceived as socially deviant (Barlow \& Durand, 1995; Miller \& Kelley, 2005). Psychologists may be particularly prone to equate social deviance with psychopathology, since there is a general clinician bias to perceive psychopathology (Garb, 1998). As noted above, clinicians have frequently considered religious beliefs and behaviors to be symptoms of psychopathology - especially the socially deviant religious beliefs and behaviors which highly religious people demonstrate (Frame, 2003; O’Connor \& Vandenberg, 2005; Wulff, 1996). It is true some socially deviant traits are also pathologically deviant. For example, criminal behavior, drug use, elevated levels of arrest, and sexual promiscuity have all been found to be positively correlated with increased psychopathology (Dishion, 2000). It has also been established empirically that deviant beliefs and behaviors are often positively correlated with each other, in both adolescents and adults (Donovan, Jessor, \& Costa, 1988; McGee \& Newcomb, 1992; Newcomb \& McGee, 1991; Osgood, Johnston, O’Malley, \& Bachman, 1998). But does evidence exist to associate high magnitudes of religiousness with psychopathology?

Some highly religious expressions are in fact associated with an increased incidence of psychopathology. Pentecostalism is often considered by mainline Protestant Christians to be a socially deviant, extreme, or unusual form of Christianity (Altemeyer \& Hunsberger, 2005; Dobbins, 2000; McCullough et al., 2000; Moriarty, 1992). In a 
review of six-month and lifetime rates of major psychiatric disorders among 853 Protestant baby boomers during wave II of the National Institute of Mental Health's Epidemiologic Catchment Area survey conducted in 1983-1984, Koenig, George, Meador, Blazer, and Dyck (1994) found young adult Pentecostals had higher rates of psychiatric disorders than people from other religious groups or nonreligious persons. Rapid religious conversion has been consistently linked to psychopathology, as people who change religions too quickly do not have adequate time to adjust to this major cultural change. Miller and Kelley (2005) noted sudden religious conversion can be associated with "a weakened sense of ego or identity, psychopathological symptomatology, or existential anxiety (Hunter, 1998; James, 1902/1985; Zinnbauer \& Pargament, 1998)" (Miller \& Kelley, 2005, p. 465). Research also exists which correlates rigid fundamentalist religious beliefs with bigotry, homophobia, prejudice, ethnocentrism, and intolerance of the beliefs of others (Altemeyer \& Hunsberger, 2005; Ferriss, 2002; Sethi \& Seligman, 1991). Furthermore, several clinical studies have found a link between hyperreligiousness - consisting of ruminations, obsessions, guilt, and scrupulosity related to religious themes - and temporal lobe epilepsy (Bear, 1982; Fedio, 1986; Hopping, 1984; Knudsen \& Bolwig, 1986; Makarec \& Persinger, 1985; Persinger, 1984; Persinger \& Makarec, 1987; Sorenson \& Bolwig, 1987). Gartner (1996) noted these studies provide "tentative but intriguing evidence" dating back to the $19^{\text {th }}$ Century "that at least some religious experience may be mediated by temporal lobe activity" (p. 200) or linked to temporal lobe epilepsy.

Since the late 1970 's, cult members have often been perceived as mentally ill victims of brainwashing. Much of this perception is due to a highly publicized case in 
which New York psychiatrist Eli Shapiro had his son Edward declared "mentally incompetent as a result of mind control" (Richardson, 1992, p. 236) because he wanted his adult son to leave a cult called the International Society for Krishna Consciousness (ISKON). Shapiro alleged that the cult used mind control techniques against Edward, which he argued made Edward incapable of managing his diabetes and a \$30,000 estate Edward would gain control of when he reached age 21. Massachusetts psychiatrist Dr. John Clark testified against Edward as an expert witness in this case, arguing Edward "is in immediate and constant danger to himself because he is mentally ill" (Richardson, 1992, p. 236) as a result of cult involvement. Dr. Clark became a prominent figure in anti-cult circles, and his anti-cult theories conceptualizing cult members as mentally ill victims of brainwashing have become widely accepted in American society and used with varying degrees of success in subsequent legal cases. His views persist in spite of the fact other mental health experts found Edward to have no evidence of mental illness, resulting in the dismissal of the incompetency petition. Dr. Clark's anti-cult theories have been largely discredited among most scholars, and Dr. Clark himself was censured by the Massachusetts Disciplinary Board of Medicine for certifying "a patient as mentally ill and dangerous without setting forth the factual basis of these conclusions" (Richardson, 1992, p. 241). Nevertheless, the stereotype persists that cult members are brainwashed and mentally ill.

Additionally, religious extremists and members of cults behaving in maladaptive, illogical, and sometimes dangerous ways have also received a great deal of media attention in the past thirty years (Frame, 2003). For example, in 2010, Terry Jones, a pastor at tiny church in Gainesville, Florida, garnered worldwide media attention and 
condemnation from many religious groups after threatening to hold a $\mathrm{Qu}$ 'ran burning to commemorate the ninth anniversary of the September 11, 2001 terrorist attacks against the United States (Gonzalez, 2010). Since 1991, the Westboro Baptist Church has picketed over 30,000 times in all 50 states, including well-publicized anti-gay protests at military funerals in the late 2000s (Fitzgerald, 2010). In 1993, an attempt by the United States Bureau of Alcohol, Tobacco, and Firearms to raid a heavily-armed ranch outside Waco, Texas which was occupied by the Branch Davidian religious cult resulted in the deaths of four agents and six cult members during the initial raid, and in the deaths of cult leader David Koresh and 75 other cult members after a 51-day siege of the ranch culminated in a fire that destroyed the religious complex (Anthony \& Robbins, 1997). In 1997, 38 members of an American religious group called Heaven's Gate committed suicide when Comet Hale-Bopp passed closest to the earth, allegedly because they believed this would enable their souls to catch a ride on a spaceship carrying Jesus, which was supposedly hiding behind the comet (Lalich, 2004). In 1978, the name Jim Jones and the People's Temple cult he founded became synonymous with mass suicide after the mass murder/ suicide of 909 members of Rev. Jim Jones' planned community Jonestown in Guyana (Klineman, Butler, \& Conn, 1980).

Excluding the young adult Pentecostals, however, these forms of religious expression do not represent authentic forms of religiousness. People who belong to a particular religious tradition would not be likely to judge these forms of religiousness to be genuine expressions of their kind of religious faith (Sanderson et al., 1999). Established religious groups often disavow any connection to religious extremists, cult members, and even their own members whose behavior becomes too outspoken or 
deviates too far from the norm, as many American Baptist churches disavowed any connection to the Westboro Baptist Church, and as churches around the world did with Terry Jones. Mainstream members of religious faiths consider behaviors such as these to be inauthentic expressions of faith, viewing them instead as attention-seeking, misguided, or crazy behaviors having only incidental or superficial similarities to the faiths of genuine religious adherents. Similarly, rapid religious converts may be seen as having converted too recently to have internalized a genuine religious faith yet. Likewise, temporal lobe epilepsy represents a medical illness whose symptoms happen to resemble some forms of religious practice, rather than an authentic form of religious expression.

Even Pentecostalism - a recognized denomination of Protestant Christianity including over 1,000,000 members (Richards \& Bergin, 2000) - is considered by some (non-Pentecostal) Christians to be an inauthentic form of Christian faith, in part because of its practice of speaking in tongues and the prohibition of dancing, movies, makeup, jewelry, and short hair among women (Altemeyer \& Hunsberger, 2005; Dobbins, 2000). Historian Michael Moriarty described Pentecostalism as "theologically thin" (Moriarty, 1992). Furthermore, closer examination of the earlier Koenig et al. (1994) finding that young adult Pentecostals have the highest rates of psychiatric disorders "revealed that the differences between religious groups on rates of psychiatric disorder were found primarily among those who attended religious services less frequently" (Koenig, 2005, p. 166) - a population whose faith frequently churchgoing Pentecostals would likely consider to be less authentic than the faith of frequent attendees. Religious people, then, often perceive unconventional religious practices as being less authentic than conventional practices. 
Sanderson and colleagues (1999) found mental health professionals, too, hold a similar perspective, viewing conventional religious practices as being positively correlated with both authenticity and mental health. Giving conventional, less conventional, and unconventional written vignettes to 67 clinical and counseling psychologists, graduate students, and therapists, they noted mental health professionals judged people with unconventional religious practices both as having less authentic religious experiences and as being less mentally healthy than people with conventional forms of faith.

When inauthentic forms of religious expression such as cults, religious extremists, rapid religious converts, patients with temporal lobe epilepsy, and infrequently churchgoing Pentecostals are excluded, then, there is little evidence to support the idea that highly religious people are actually more likely to experience different or more severe forms of psychopathology, or to have worse prognoses for therapy, than people with lower magnitudes of faith. In fact, the preponderance of evidence suggests high levels of religiousness are negatively correlated with psychopathology, or at worst are unrelated to mental illness. High magnitudes of religiousness may actually be less pathological than lower magnitudes (Miller \& Kelley, 2005; Oman \& Thoresen, 2005). Lindenthal, Myers, Pepper, and Stern (1970) presented longitudinal data supporting an inverse, "dose-response" relationship between religious affiliation and psychological impairment: greater religiousness was associated with proportionally less psychopathology. Several decades of empirical research reviewed by Koenig (1998, 2005) found strong positive relationships between religious involvement and mental health. 
Some evidence even suggests statistically deviant forms of religiousness (high and low religiousness) may be less pathological than statistically normative forms (moderate religiousness). A small but growing number of studies have shown a curvilinear relationship between religiousness and psychopathology. The majority of studies examining faith have measured religiousness as a categorical variable, as simply present or absent, or as occurring in high or low levels. When studies instead examine religiousness as a continuous trait varying from high to moderate to low levels, moderate levels of religiousness have been found to be more highly correlated with undesirable traits than either high or low levels of religiousness. For example, moderately religious people are more likely to fear death (Wink \& Scott, 2005), to experience higher levels of depression (Eliassen, Taylor, \& Lloyd, 2005), and to hold racist views (Perkins, 1992) than people high or low in religiousness. Likewise, Miller and Kelley (2005) observed that a close examination of correlational data suggests a curvilinear relationship between religiousness and mental health on overall distress (Ross, 1990), depressive expression (Schnittker, 2001), general anxiety (Koenig, Ford, George, Blazer, \& Meador, 1993), and death anxiety (Pressman, Lyons, Larson, \& Gartner, 1992), as well as over the course of the lifespan (Ingersoll-Dayton, Krause, \& Morgan, 2002). In other words, highly religious people may actually have lower base rates of mental health issues than moderately religious people do, and the same base rates of mental illness as are found among their nonreligious counterparts. Conversely, Miller and Kelley (2005) noted in some cases, "those at the far ends of the religious continuum are the most susceptible to suffering, and those that adhere to a more moderate practice or belief system show better functioning" (p. 462). In some instances, then, moderately religious people may enjoy 
less psychopathology than people with low or high religiousness. It is possible there is an empirical relationship between level of religiousness and mental health. Since most past studies have merely measured religiousness categorically and few have measured it as a continuous variable, this relationship has yet to be established. It would be prudent, therefore, for future studies of religious clinical judgments to differentiate between different levels of religiousness.

\section{Religion and Obsessionality}

There are some extreme forms of religious expression which can resemble symptoms of psychopathology - especially anxiety disorders in general, and obsessivecompulsive disorder in particular. Recall the stereotype suggested by Gartner and colleagues (1990a, 1990b) and by Yossifova and Loewenthal (1999) that religious behavior is associated with obsessional symptoms. Rituals can be an important part of religious life, but when they are overdone, they can "metastasize into OCD-like presentation" (Miller \& Kelley, 2005, p. 471). For example, a moderately religious person may pray several times a day, but an extremely religious person who literally interprets the invocations of the Bible or the Koran to be perpetually in prayer might pray dozens or even hundreds of times a day. In Islam, ritual cleaning is necessary to prepare for salat (prayer five times daily), and the notion of waswas (the devil's whispering) can result in a devout Muslim performing repeated acts of cleaning and absolution. Similarly, Hindus experiencing suci bhay (purity mania) may require holy water blessings and markedly limit what they touch, which can interfere with their daily lives (Miller \& Kelley, 2005). Taken out of context, these rituals can sometimes resemble OCD symptoms. 
As early as 1907, Freud noted a remarkable similarity between religious practices and obsessive actions. Both involve ritual, are executed to the exclusion of other behaviors, and invoke guilt if neglected (Greenberg, 1984). He believed both were used by the psyche to ward off sexual or aggressive instincts (Lewis, 1998; Yossifova \& Loewenthal, 1999), and in 1927, he wrote, “One might venture to regard obsessional neurosis as a pathological counterpart of the formation of a religion, and to describe that neurosis as an individual religiousness, and religion as a universal obsessional neurosis" (Freud, 1927; cited in Greenberg, 1984, p. 524). In OCD, religious themes are often salient, and religious guilt can manifest itself as an obsessional symptom (Higgins \& Pollard, 1992; Yossifova \& Loewenthal, 1999). Formally, "religious scrupulosity" is a term which has been coined to refer to the ritualistic obsessions and compulsions which are often found in religious people with OCD (Greenberg \& Witztum, 1994; Miller \& Kelley, 2005). Greenberg and Shefler (2002) observed both religious rituals and OCD symptoms have superficial similarities. Like OCD, religious rituals often need to be performed repeatedly and in a certain order, and in both OCD compulsions and many religious rituals, precision is valued, while leaving out prescribed steps is often cause to repeat the action over again. Cleanliness is also highly valued in several different religions, as well as in many forms of OCD. Consequently, the stereotype linking religiousness and OCD symptoms persists. Thus it is not surprising many people have come to view religion itself, or at least highly religious people, as pathological.

There is mixed evidence to support the stereotype that religiousness is correlated with anxiety or obsessional symptoms. Most reviews have found negative or no correlation between religiousness and anxiety in general (59 studies cited in Koenig, 
1998; 76 studies cited in Koenig, 2005) or between religiousness and OCD in particular (Greenberg \& Shefler, 2002; Hermesh, Masser-Kavitsky, \& Gross-Isseroff, 2003; Tek \& Ulug, 2001; Okasha, Lotaief, Ashour, El Mahalawy, Seif El Dawla, \& El-Kholy, 2000). Koenig (2005) reported, of 76 studies published before the year 2000 which examined the relationship between religion and anxiety, 35 found a negative correlation between level of religiousness and level of anxiety, while 24 found no association. Only 10 studies - all of them cross-sectional - found a positive correlation between anxiety and religion, and Koenig (2005) cited longitudinal studies which found religious interventions to reduce anxiety, suggesting anxiety may have led the people in these 10 studies to become more religious, rather than religion resulting in greater anxiety symptoms. Several studies investigating the relationship between religiousness and the diagnosis of ObsessiveCompulsive Disorder have found no relationship between religious beliefs or behavior and any clinical feature of OCD, including religious obsessions (Lewis, 1998; Tek \& Ulug, 2001; Yaryura-Tobias, Grunes, Todaro, McKay, Neziroglu, \& Stockman, 2000). As mentioned previously, recent literature reviews on anxiety and religion suggest religious people are not more susceptible to anxiety than their nonreligious counterparts, though those with anxiety disorders may be more religious than other people (Miller \& Kelley, 2005). Some reviews on religion and mental health have reported a small positive correlation between general symptoms of anxiety and religion (e.g., Harris, Schoneman, \& Carrera, 2002; Miller \& Kelley, 2005). Notably, though, the apparent relationship between these two variables disappeared when controlling for socioeconomic status, social support, disability, and chronic illness (Koenig, Ford, George, Blazer, \& Meador, 1993; Koenig, George, Blazer, Pritchett, \& Meador, 1993). Furthermore, while some 
authors have speculated OCD is more common in highly religious societies

(Chackraborty, 1975), Greenberg and Shefler (2002) have effectively argued against this point. They observed that, while this hypothesis has not yet been directly tested, "the findings of a remarkably similar prevalence rate for OCD in a range of countries and cultures argue indirectly against such a claim" (p. 123).

Some studies, however, have found a correlation between religiousness and obsessionality. In a sample of OCD patients, Steketee, Quay, and White (1991) found a significant positive association between religious identification and severity of OCD symptoms, while Alonso and colleagues (2001) and Mataix-Cols and colleagues (2002) both found patients who had higher levels of obsessions with religious content had poorer outcomes from treatment than either patients who had higher levels of obsessions without religious content or patients who had lower levels of obsessions, even after controlling for the initial severity of patient symptoms (Alonso, Menchon, Pifarre, Mataix-Cols, Torres, Salgado, \& Vallejo, 2001; Mataix-Cols, Rosario-Campos, \& Leckman, 2002). Similarly, Abramowitz, Deacon, Woods, and Tolin (2004) found highly religious undergraduates reported more obsessional symptoms than students who were moderately religious or atheist. Notably, though, when Lewis and Maltby (1995) took into account gender, they found in two research studies sampling adults from the United Kingdom and Northern Ireland, obsessional characteristics were associated with both a favorable attitude toward religion and frequency of religious behavior, but only in females. Koenig (2005) also noted religious participants might be expected to score more highly on OCD scales than nonreligious participants do, since many of these scales include questions assessing traditional religious values such as attention to detail and personal 
responsibility. Furthermore, Fontana (1980) argued obsessional personality traits (consisting of orderliness, rigidity, and overemphasis on hygiene and self-control) differ from obsessional symptoms (including obsessive thoughts, compulsive behaviors, ritual behaviors, guilt, and indecision). Based on this distinction between traits and symptoms, Lewis and Maltby (1995) observed that research consistently shows positive associations between religious attitude and obsessional personality traits (Juni \& Fischer, 1985; Lewis, 1994; Lewis \& Joseph, 1994a; Lewis \& Maltby, 1992, 1994), as well as between religious practice and obsessional personality traits (Juni \& Fischer, 1985; Lewis \& Joseph, 1994b). On the other hand, Lewis and Maltby (1995) noted this same body of research consistently shows no correlation between religious attitude and obsessional symptoms (Lewis, 1994; Maltby, McCollam, \& Millar, 1994), while yielding "insufficient data" (Lewis \& Maltby, 1995, p. 105) on any relationship between religious practice and obsessional symptoms (c.f., Lewis, 1994; Lewis \& Joseph, 1994b). Thus a relationship between religion and obsessional personality traits has been found in a few cases (though this may be due to measurement bias rather than any genuine association), while research does not support any association between religion and obsessional symptoms - the kind of symptoms required for a clinical diagnosis of OCD.

\section{Evidence of Bias}

In short, then, the belief that high magnitudes of religiousness are associated with pathology may be an unfounded stereotype because it is not supported and in many cases contradicted by the empirical research literature. This literature strongly suggests base rates of psychopathology do not differ between the religious and the nonreligious, or among people of varying magnitudes of religiousness. Nevertheless, clinical judgment 
research like the aforementioned studies by Sanderson et al. (1999), O’Connor and Vandenberg (2005), and Gartner et al. (1990a, 1990b) reveals clinicians may formulate more pathological diagnostic and prognostic judgments for religious clients, especially for clients who are very religious. Since these types of clinical judgments are contraindicated by the empirical research literature, they presumably represent a potential clinical judgment faith bias:

Clinical Judgment Faith Bias: a proposed tendency for clinicians to make more pathological clinical judgments for clients with higher magnitudes of faith than for otherwise identical clients with socially normative faith Socially normative faith refers to forms of faith that the majority of people hold and which conform to social or conventional norms, such as moderate or low religiousness or spirituality.

\section{Stereotyping, Salience Bias, and the Representativeness Heuristic}

Three phenomena from behavior decision-making theory may help to explain why clinical judgment faith bias might occur: stereotyping, salience bias, and the representativeness heuristic. All three are heuristics or cognitive shortcuts clinicians sometimes employ in making clinical judgments. Any one of these phenomena in isolation may be insufficient to lead to bias, but all three working in concert could lead clinicians to make clinical judgment errors with religious clients.

First, three stereotypes bear mentioning. One stereotype exists associating social deviance with psychopathology (Barlow \& Durand, 1995). Psychologists may be particularly prone to equate the two, since there is a general clinician bias to perceive psychopathology (Garb, 1998). Hence, psychologists may be more likely to perceive 
socially deviant beliefs and behaviors as psychopathological. A second stereotype exists associating religiousness with psychopathology - namely, that religious people are mentally ill in some way, or that religiousness itself is a symptom of mental illness (c.f., Ellis, 1980; Freud, 1907/1959, 1927; Miller \& Kelley, 2005; Richards \& Bergin, 2000). Furthermore, since Freud, a third stereotype has existed among clinicians and lay persons alike equating religious behaviors with obsessional symptoms signifying mental problems (c.f., Greenberg, 1984; Tek \& Ulug, 2001; Yossifova \& Loewenthal, 1999). All three stereotypes are untrue, but any one could lead an unsuspecting clinician who believes it to make an erroneous judgment, if the stereotype comes to mind at the wrong time and the clinician does not discount it.

Second, one or more of these stereotypes may become salient to the clinician. Salience refers to the perceived distinctiveness of a specific quality or characteristic. Salience bias occurs when clinicians make attributions based on the perceptual information which is most distinctive rather than the data which is most pertinent to the decision at hand. According to Taylor (2006), "Salience biases refer to the fact that colorful, dynamic, or other distinctive stimuli disproportionately engage attention and accordingly disproportionately affect judgments" (p. 192). Socially deviant forms of religiousness such as highly religious behavior may make salient the first two of the previously mentioned stereotypes (religion is associated with psychopathology, and deviance is linked to psychopathology), while highly religious behavior in a client presenting with obsessional symptoms may make salient the third stereotype (religion is related to obsessional symptoms). Salience bias may then lead clinicians to make 
judgments based on these stereotypes rather than on information more pertinent to these judgments.

Finally, the representativeness heuristic is a cognitive shortcut clinicians use when judging whether an object fits into (or is representative of) a particular category or group (Baron \& Byrne, 1997; Garb, 1998; Kahneman \& Tversky, 1973; Nisbett \& Ross, 1980) - a set of 'goodness of fit criteria' (Morrow \& Deidan, 1992). This heuristic refers to a "strategy for making judgments based on the extent to which current stimuli or events resemble other stimuli or categories" (Baron \& Byrne, 1997, p. 83) without properly considering their base rates, or how often these stereotypes are actually true. When clinicians misuse the representativeness heuristic, they invoke stereotypes or schemata without thinking about base rates, which increases their chances of making a clinical judgment error referred to by Bar-Hillel (1980) and Lopez (1989) as the base rate fallacy. The representativeness heuristic may lead clinicians to ignore base rates and decide the three aforementioned stereotypes are representative of religious people - particularly in situations in which socially deviant forms of religiousness make these stereotypes salient. If clinicians believe that religion is associated with psychopathology, that deviance is related to psychopathology, and that religion is linked to obsessional symptoms, these clinicians are then more likely to make pathological judgments of these clients, while ignoring the relevant base rates of equivalent levels of pathology among highly religious people as among nonreligious people - base-rate information which should lead them to discount these stereotypes. The representativeness heuristic, then, refers to a cognitive shortcut people use when judging whether an object or event is representative of a 
particular category or group without considering the base rates at which that object or event occurs in that group.

When the clinicians in many of the aforementioned studies gave more pathological diagnostic and prognostic ratings to moderately and highly religious clients, it is likely they were not attending to the relevant base rates of these disorders and were, instead, invoking the representativeness heuristic and making judgments based on stereotypes rather than the best available data. This may have occurred especially when religiousness became salient to these clinicians, such as when clients were perceived as socially deviant or as presenting with anxiety symptoms, leading to salience bias. With stereotyping, salience bias, and the representativeness heuristic coming into play, clinicians may find themselves prone to clinical judgment faith bias.

Several factors may influence whether or not these cognitive heuristics are activated - and, hence, whether a particular clinician actually makes a clinical judgment error with a given client. Magnitude of religiousness (high, medium, or low) and the presence or absence of obsessional symptoms are likely to be two of those factors. Notably, four other variables bear scrutiny, because of the likelihood they may interact with magnitude of religiousness to activate these cognitive heuristics. These variables are client spirituality as distinct from client religiousness (Feeser, 1997; Zinnbauer \& Pargament, 2005), clinician multicultural competence (Constantine, 2002; Ponterotto, Casas, Suzuki, \& Alexander, 2001; Richeson \& Nussbaum, 2004), clinician faith (Kahle, 1998; Lewis \& Lewis, 1985; Worthington et al., 1996), and clinician view of faith helpfulness (Hecker, Trepper, Wetchler, \& Fontaine, 1995; Hillowe, 1985; Walker, Gorsuch, \& Tan, 2004; Worthington et al., 1996). Each is explored here. 


\section{Weaknesses in Previous Clinical Judgment Faith Bias Studies}

Few empirical studies have investigated clinical judgment faith bias, this hypothesized tendency for clinicians to make more pathological clinical judgments for clients with higher magnitudes of faith than for otherwise identical clients with socially normative faith. Moreover, those previous studies addressing this hypothesis have had numerous weaknesses, including the treatment of religion as a categorical variable, the comparison of qualitatively different types of religious expressions, the investigation of inauthentic kinds of religiousness, and the neglect of spirituality as a distinct variable from religiousness.

First, many of them treated religion as a categorical or dichotomous trait, examining merely the presence or absence of religious ideation or behavior rather than differing levels of religiousness. Gerson, Allen, Gold, and Kose (2000) gave participants only two vignettes - a religious vignette and a nonreligious vignette - and Hillowe's (1985) respondents rated religious and nonreligious versions of a depression vignette and an anxiety vignette. Lewis and Lewis (1985) gave psychologists tapes of therapists interviewing a depressed female client who made either religious or nonreligious statements, while Marcinkowski (1993) gave subjects client scripts in which client theism was either absent or present in statements endorsing a belief in God. Similarly, the religious vignettes examined by Gartner et al. (1990a, 1990b) consisted of fundamentalist Christian and Atheist vignettes.

Second, those studies examining religiousness as a continuous trait often looked at different levels of qualitatively different types of religious expressions. For example, O'Connor and Vandenberg (2005) investigated Catholic, Latter Day Saint, and Nation of 
Islam as faiths with differing levels of social normativity. These three forms of religiousness, however, are so qualitatively different it is difficult to tell whether clinicians responded to the magnitude of the faith or to the specific content of the religious beliefs and practices. Similarly, Jones's (1991) unpublished doctoral dissertation investigated clinical judgments made about nonreligious, very religious, and religiously doubtful vignettes, but the very religious vignettes (describing strong belief) may have been depicting something altogether different from the religiously doubtful vignettes (describing doubt and uncertainty of belief).

Furthermore, some of the forms of faith investigated in previous research were extremely socially deviant. Ostensibly, this was done so the different levels of faith would be differentiable when measured by instruments with low to moderate sensitivity, but elevating the level of social deviance portrayed in the highly religious vignettes had the unfortunate side-effect of portraying behaviors and beliefs that were so socially deviant as to be inauthentic, artificial fabrications of faith which did not represent realworld expressions of religiousness or spirituality. For example, Sanderson et al. (1999) had 67 mental health professionals rate the degree of mental health portrayed in 18 case vignettes which varied on the degree to which the portrayed religious experience differed from the norm. Three levels of deviance from the norm were portrayed: conventional, less conventional, and unconventional practices. The unconventional religious practices which were depicted included giving away all of their children's possessions, having a vision in which the person is told they are Christ, hearing the voice of God telling the person to sacrifice their child, cutting off their hand after reading a Biblical passage to "cut off the hand that has sinned," receiving a message from Jesus through the television, 
and experiencing the presence of Elvis. It seems unlikely many religious adherents would consider these practices to be authentic expressions of their religious faiths. Though Sanderson et al. (1999) succeeded in capturing unconventional religious practices, such behaviors do not represent authentic, real-world religious behaviors.

Another notable weakness in much of the research literature on clinical judgment faith bias is researchers' almost exclusive examination of client religiousness. Virtually no studies have examined the similar construct of client spirituality. Client spirituality is a construct which is highly related to yet distinct from client religiousness, and these two constructs may have different effects on a potential clinical judgment faith bias (Feeser, 1997; Zinnbauer \& Pargament, 2005). Noting the dearth of studies treating spirituality and religiousness as distinct constructs, Feeser (1997) examined both as separate variables. With some notable exceptions (including Feeser, 1997; Shafranske \& Gorsuch, 1984; and Shafranske \& Malony, 1990), few studies have treated these constructs as distinct variables, yet doing so could improve upon the accuracy of existing estimates of any clinical judgment faith bias that does exist. This is a shortcoming in existing research on the subject.

\section{Client Religiousness, Spirituality, and Clinical Judgment Faith Bias}

One variable which may influence whether client faith influences clinicians' diagnostic and prognostic judgments is the variable of client spirituality, as distinct from client religiousness. Spirituality and religiousness may be distinct constructs which may have different effects on any clinical judgment faith bias that does exist, so these two variables bear scrutiny as separate constructs. 
There is a growing recognition in the research literature that religiousness and spirituality may be distinct constructs. Beck (1986), Booth (1984), Elkins, Hedstrom, Hughes, Leaf, and Saunders (1988), Ingersoll (1994), Legere (1984), Shafranske and Gorsuch (1984), Shafranske and Malony (1990), Turner, Lukoff, Barnhouse, and Lu (1995), and Zinnbauer and Pargament (2005) are just a few of the many authors who have used these two terms to refer to concepts which are related and overlapping, but nevertheless distinct. Rose et al. (2001) surveyed priests, monks, rabbis, imams, Sikhs, and other religious leaders from Christianity, Islam, Buddhism, Judaism, and Hinduism as well as several "non-tradition" psychologists, teachers, and authors, and he concluded from the many responses he received that while the terms religiousness and spirituality have similar meanings, most respondents considered them to be different concepts (except for Muslim respondents). Likewise, Cook, Borman, Moore, \& Kunkel (2000) concept-mapped 16 college students' conception of religiousness and spirituality and found $75 \%$ of their sample conceptualized these terms as different ideas.

A growing body of empirical research has provided evidence that spiritual and religious scales measure distinct constructs. For example, in a meta-analysis of the relationship between the five-factor model (FFM) of personality and measures of religiousness and spirituality, Saroglou (2002) found religiousness scales appear to relate more to the FFM dimensions of Conscientiousness and Agreeableness, while spirituality scales seem to be more related to the FFM dimensions of Openness and Agreeableness, suggesting while religiousness and spirituality share something in common, namely, "a compassionate attitude toward others," religiousness involves "more of the dutiful, procedural aspects of faith involvement," while spirituality involves "a seeking, curious 
attitude toward the transcendent" (Piedmont, 2005, p. 263). Similarly, in a joint factor analysis of the FFM and the Brief Multidimensional Measure of Religiousness/ Spirituality, Piedmont, Ciarrocchi, Dy-Liacco, Mapa, and Williams (2003) found religiousness and spirituality to be highly correlated factors, distinct from one another and from the five factors of personality.

In spite of the amount of research and conceptualization devoted to these two constructs, the terms religiousness and spirituality continue to be somewhat poorly defined in the psychology research literature. Authors seem to have a sense of what spirituality and religiousness mean connotatively, yet operationalizing them denotatively has been highly problematic, as there is a notable lack of consensus as to what these two constructs mean (McKinnon, 2002). This lack of consensus impedes research on the impact of religious and spiritual issues on counseling and may hinder the provision of adequate mental health services to people with faith values (Dyson, Cobb, \& Forman, 1997).

The terms spirituality and religiousness frequently co-occur in the research literature. Of the two terms, religiousness is the oldest, while spirituality has only emerged within the last hundred years or so, as a Western concept (Hill, Pargament, Hood, McCullough, Swyers, Larson, \& Zinnbauer, 2000). Some authors have bifurcated religion and spirituality into the institutional versus the individual. This is a useful heuristic, but it ignores the institutional elements of spirituality and the individual elements of religion (Dyson et al., 1997; Hill et al., 2000). Still, most researchers agree spirituality and religiousness are related but distinct constructs, though there is much less consensus on how, precisely, they are related and how they are distinct. 
Religiousness seems to be the term used most often in the research literature, though the term spirituality has appeared in exponentially increasing numbers of articles since the early 1990's and may be replacing religiousness as the preferred term in the mental health literature (Harris, Howell, Spurgeon, Sirken, McConnell, \& Coulter, 2006). Religiousness has been defined in many different ways (Zinnbauer \& Pargament, 2005), including as "a system of beliefs in a divine or superhuman power, and practices of worship or other rituals directed towards such a power" (Argyle \& Beit-Hallahmi, 1975, p. 1), "a set of symbolic forms and acts that relate man to the ultimate conditions of his existence" (Bellah, 1970, p. 21), "a covenant faith community with teachings and narratives that enhance the search for the sacred" (Dollahite, 1998, p. 5), "systems of belief in and response to the divine, including the sacred books, cultic rituals, and ethical practices of the adherents" (O'Collins \& Farrugia, 1991, p. 203), and “commitments of beliefs and practices characteristic of particular traditions" (Peteet, 1994, p. 237). Often, in defining religiousness, writers use the word in its own definition (Shafranske \& Malony, 1990). Historically, religiousness has been designated in much simpler ways. According to Hill et al. (2000), religiousness has typically been defined in three different ways: (1) something involving a higher supernatural power, (2) a feeling present in a perceiver of a higher power, or (3) ritual behavior done to show regard for a higher power (Hill et al., 2000). Some researchers, though, have come up with as many as 12 different dimensions or characteristics of religiousness (Fontana, 2003). It is difficult, then, to discern consensus in the conceptualization of this multidimensional construct. There are, however, some patterns in the literature. Researchers who are writing on the construct of religiousness seem to refer to the construct as having to do with four different 
phenomena: (1) beliefs, (2) practices, (3) God or a higher power, and (4) organized religion (c.f. Argyle \& Beit-Hallahmi, 1975; Clark, 1958; Dollahite, 1998; James, 1902/1961; O’Collins \& Farrugia, 1991; Peteet, 1994).

Similarly, spirituality has been defined in many different ways (Dyson et al., 1997). Like religiousness, spirituality has also been defined in a multitude of different ways (Zinnbauer \& Pargament, 2005), including as "the presence of a relationship with a Higher Power that affects the way in which one operates in the world" (Armstrong, 1995, p. 3), "the search for existential meaning" (Doyle, 1992, p. 302), "a subjective experience of the sacred" (Vaughan, 1991, p. 105), "a transcendent dimension within human experience... discovered in moments in which the individual questions the meaning of personal existence and attempts to place the self within a broader ontological context" (Shafranske \& Gorsuch, 1984, p. 231), and "that vast realm of human potential dealing with ultimate purposes, with higher entities, with God, with love, with compassion, with purpose" (Tart, 1975, p. 4). With all these differing ideas as to what constitutes the multifaceted construct of spirituality, it is as difficult to devise a working definition of this construct as it is to arrive at a working definition of religiousness - perhaps even more difficult, because of the overlap between the two concepts. Fortunately, there are some patterns in the research literature. A literature review by Dyson, Cobb, and Forman (1997) revealed several themes common to different conceptualizations of spirituality: a search for meaning in life, the need for hope, relatedness or connectedness to others, a set of religious or non-religious beliefs, and expression of spirituality in multiple ways. Other researchers who are writing on the construct of spirituality seem to refer to spirituality as having to do with four different phenomena: (1) an internal belief system (c.f., Burkhart 
\& Solari-Twadell, 2001; Cook et al., 2000; Emmons \& Crumpler, 1999; Hawks, 1994),

(2) the construction of meaning (c.f., Benjamin \& Looby, 1998; Burke \& Hackney, 1999;

Burkhart \& Solari-Twadell, 2001; Erickson, Hecker, Kirkpatrick, Killmer, \& James, 2002; Shafranske \& Gorsuch, 1984), (3) a relationship (c.f., Burke \& Hackney, 1999;

Burkhart \& Solari-Twadell, 2001; Dyson et al., 1997), and (4) a higher power or an

ultimate reality (c.f., Benjamin \& Looby, 1998; Burke \& Hackney, 1999; Sigmund, 2002; Wolf \& Stevens, 2001).

One recent attempt to trace the outline of the nature of both spirituality and religiousness involved the introduction of yet another faith term: the sacred. In 1997, there was a series of conferences in which 60 professionals - psychologists, doctors, neuroscientists, and substance abuse counselors - attempted to define religiousness and spirituality. Based on this conference series, Hill et al. (2000) proposed definitional criteria for future research - not definitions, but a set of criteria for judging operational definitions of spirituality and religiousness. Paraphrased, Hill et al.'s (2000) working definition of spirituality is a search for the sacred, and their working definition of religiousness is a search for the sacred (or non-sacred things) in a socially-sanctioned context that typically facilitates searches for the sacred, where the sacred is an ultimate, transcendent, or divine being, reality, or truth as perceived by an individual. Therefore, both spirituality and religiousness involve a person's relationship with the sacred, but spirituality is often a personal search, while religiousness typically requires an institutional or cultural context. Similarly, in a recent review of the terms spirituality and religiousness, Zinnbauer and Pargament (2005) reviewed dozens of definitions of these two terms appearing in the psychology of religion literature and concluded their review 
by presenting two sets of definitions - one from each author - reflecting two overall trends in the literature. Both authors defined spirituality as "a search for the sacred" (Zinnbauer \& Pargament, 2005, pp. 35, 36), where a search refers to the pursuit of "a particular set of valued, meaningful, or ultimate concerns" (p. 33), and "the sacred refers to concepts of God, higher powers, transcendent beings, or other aspects of life... that take on divine character and meaning through their association with or representation of the holy" (p. 34). The two authors differed, however, in how each defined religiousness. Pargament defined religiousness as "a search for significance in ways related to the sacred" (Zinnbauer \& Pargament, 2005, p. 36), conceptualizing religiousness as having a broader set of ends than spirituality, and reflecting a major trend in the psychology of religion literature defining religiousness as the broader construct. On the other hand, Zinnbauer defined religiousness as "a personal or group search for the sacred that unfolds within a traditional sacred context” (Zinnbauer \& Pargament, 2005, p. 35), conceptualizing religiousness as a contextualized or organized form of spirituality, and reflecting another major trend in the literature defining spirituality as the broader construct. Zinnbauer's definition echoed Hill et al.’s (2000) definitional criteria. Here, Hill et al.'s (2000) working definitions of spirituality and religiousness have been adopted, because of the way in which they were developed, the number of prominent psychology of religion experts involved in their development, their comprehensiveness, and their similarity to other accepted definitions of these terms (e.g., Zinnbauer \& Pargament, 2005). 
Religiousness: a search for the sacred (or non-sacred things such as social contact or appearing virtuous to your neighbors) in a traditional institutional context that typically facilitates searches for the sacred

Spirituality: a personal search for the sacred which may or may not occur in a traditional institutional context that typically facilitates searches for the sacred

Again, the sacred refers to an ultimate, transcendent, or divine being, reality, or truth as perceived by an individual.

Along with religiousness and spirituality, the term faith sometimes also appears in the literature to describe similar phenomena. When this term is used, however, it is usually used as a synonym for spirituality (e.g., "my personal faith in God"), as a synonym for religiousness (e.g., "my faith is Hinduism"), or interchangeably with either term. Therefore, the term faith is conceptualized here as being synonymous with religiousness, spirituality, or both.

The clinical judgment literature on religion and spirituality is not instructive as to whether client spirituality (as distinct from client religiousness) affects the clinical judgments clinicians make of clients. Logically, it seems reasonable to expect client spirituality would lead to a similar bias as client religiousness would - that, in making judgments for spiritual clients, clinicians would be affected by the same type of bias as they might be affected by in making judgments for religious clients. If this is the case, then clinicians may sometimes be prone to clinical judgment bias with spiritual clients as well as with religious clients. Clinicians might tend to give spiritual clients more pathological diagnoses and prognostic ratings than identical secular clients. Returning to 
the issue of social deviance, though, clinicians may consider spirituality to be more socially acceptable - less socially deviant - than religiousness (Bergin, 1991; Kahle, 1998). Internalized spiritual beliefs in the absence of religious rituals or practice (e.g., believing in God and living a moral life in accordance with this belief while infrequently attending church and never praying publicly) may be viewed as being more normative than highly religious behavior, which may be viewed as socially deviant (e.g., attending church three times per week and overtly bowing one's head and folding one's hands before every meal). Alternately, internalized spiritual beliefs are difficult to observe behaviorally, while overt displays of religious ritual are readily observable. If either of these are the case, then high levels of client spirituality may be less likely to activate the representativeness heuristic than high levels of client religiousness, and clinicians may not show clinical judgment faith bias with spiritual clients.

Feeser's (1997) dissertation specifically examined counseling psychologists' ratings of the perceived attractiveness and overall prognosis of religious, spiritual, and secular cases. Feeser (1997) found client spirituality as distinct from client religiousness did not affect the prognostic judgments counseling psychologists made. Unfortunately, Feeser (1997) did not examine any other clinical judgments of pathology, including diagnostic judgments. Also, with the exception of Feeser (1997), spirituality has rarely been measured as a separate construct from religiousness in the clinical judgment literature. Thus it is unclear on the basis of one study how spirituality as distinct from religiousness might influence the diagnostic and prognostic judgments clinicians make. Whichever is the case, though, measuring spirituality and religiousness as distinct 
constructs should improve upon the accuracy of existing estimates of any clinical judgment faith bias that does exist.

\section{Clinician Multicultural Competence and Faith Bias}

Another variable which may influence whether or not client faith influences clinicians' judgments is the variable of clinician multicultural competence. Not all clinicians may be equally susceptible to making biased religious and spiritual clinical judgments because of individual differences in the way they process clinical information. Multicultural competence, specifically, may have a moderating effect on any clinical judgment faith bias that exists, so this variable also bears scrutiny.

The Handbook of Multicultural Counseling conceptualizes multicultural counseling as "counseling that takes place between or among individuals from different cultural backgrounds" (Jackson, 1995, p. 3). Multicultural counselors are required to attend to group diversity "in terms of race, ethnicity, sexual orientation, religion, geographic region, and immigration status" (Ponterotto et al., 2001, p. 1). According to Pederson (1990), multiculturalism incorporates a broad view of culture, including “ethnographic variables such as ethnicity, nationality, religion, and language, as well as demographic variables such as age, gender, and place of residence, [and] status variables such as social, economic, and educational factors" (p. 93). A great deal has been written about this concept, and much of it refers back to the issue of competencies as outlined by Sue and colleagues in 1982 and in 1992. Sue, Bernier, Durran, Feinberg, Pederson, Smith, and Vasquez-Nuttall (1982) maintained multicultural competence consists of three dimensions: beliefs and attitudes, knowledge, and skills. Culturally competent people are aware of their own beliefs and attitudes about culture, possess knowledge about other 
cultures, and learn skills for working with people from other cultures. Ten years later, Sue, Arredondo, and McDavis (1992) added to those three dimensions three characteristics multiculturally competent counselors possess: an awareness of one's own cultural values and biases, an awareness of the worldview of others who are culturally different, and a working knowledge of culturally appropriate intervention strategies. Broadly defined, then, the term multicultural competence refers to those beliefs and attitudes, knowledge, and skills that lead to an awareness of one's own cultural values, the worldviews of others, and appropriate interventions for people from different cultures (Sue et al., 1982, 1992), where the term culture refers to race, ethnicity, nationality, age, gender, sexual orientation, religion, language, geographic region, immigration status, and disability (Pederson, 1990; Ponterotto et al., 2001).

Many psychologists, counselors, and other scholars believe faith is an important part of multicultural competence (Feeser, 1997; Frame, 2003; Hall, 2001; Kelly, 1995; McLennan, Rochow, \& Arthur, 2001; Pate \& Bondi, 1992; Pederson, 1990; Richards \& Bergin, 2000). Pate and Bondi (1992) view "religious beliefs and practice" as "an integral aspect of multicultural awareness" (p. 108). According to Arredondo (1999), religion and spirituality are not primary aspects of multicultural competence but are nevertheless part of the secondary dimensions of identity to which multiculturally competent counselors must attend. The American Psychological Association has formally included faith in its multicultural guidelines:

Psychologists are aware of and respect cultural, individual, and role differences, including those based on age, gender, gender identity, race, ethnicity, culture, national origin, religion, sexual orientation, disability, language, and 
socioeconomic status and consider these factors when working with members of such groups. (APA, 2002, p. 4, emphasis added)

Likewise, both the first and second editions of the Handbook of Multicultural Counseling include spirituality and religion as elements of multiculturalism and acknowledge they comprise together a major source of diversity for people from every culture, though neither edition discusses religion or spirituality extensively (Ponterroto et al., 1995, 2001). Thus, multiculturally competent counselors are expected to attend to client faith.

Multiculturally competent counselors are less likely than other clinicians to make clinical judgments influenced by race bias (Constantine, 2002; Ponterotto et al., 2001; Richeson \& Nussbaum, 2004). They are more familiar with how Black clients present in counseling, including ostensibly the relevant base rates of Black psychopathology and perhaps also the research literature demonstrating race biases in clinical judgment. In other words, they have racial multicultural competence and are competent in dealing with the cultural issue of race, and their racial multicultural competence has a moderating effect on racial clinical judgment biases whereby they are less likely to make judgments reflecting a race bias.

Although this has not been empirically established, multiculturally competent counselors may also be less likely than other clinicians to make clinical judgments influenced by client faith. In other words, they may also have faith multicultural competence and may be more competent in dealing with the cultural issues of religiousness and spirituality. Multiculturally competent counselors may be competent in dealing with racial issues (in the traditional sense of the term multicultural - c.f., Holcomb-McCoy, 2000; Kiselica, 1998), or they may be more broadly competent in 
dealing with a number of cultural issues, including race, ethnicity, language, sexuality, gender, and faith (in the broader sense of the term multicultural - c.f., Pederson, 1990; Ponterotto et al., 1995, 2001). In the latter case, they would be expected to make unbiased clinical judgments of spiritual and religious clients because of their competence in dealing with issues of faith. They hold to a broad conceptualization of multicultural competence and view religiousness and spirituality as important and salient parts of the cultural backgrounds of their clients, so they are better able to take these issues into consideration. In the former case, they are not specifically proficient in addressing faith, but they are adept in handling racial issues. Their racial competence might have a collateral effect on their ability to deal with religious and spiritual issues and might translate to a similar faith competence. If either of these cases is true, then multiculturally competent clinicians may be less likely than others to allow stereotyping, salience bias, and the representativeness heuristic to interfere with their clinical decision-making and may be less prone than other clinicians to any clinical judgment faith bias that exists.

Alternatively, faith may not be part of multicultural competence. Instead, some writers (e.g., Richards \& Bergin, 2000; Stanard, Sandhu, \& Painter, 2000) argue faith competence and multicultural competence are distinct competencies, separate and independent of one another. Stanard et al. (2000) described spirituality as an emerging fifth force in counseling and psychotherapy, emerging from and related to but distinct from the four earlier movements of psychoanalysis, behaviorism, humanism, and multiculturalism. As evidence, they cited "growing interest in psychospiritual interventions and inclusion of spirituality and religious problems in the DSM-IV" (Stanard et al., 2000, p. 209). Richards and Bergin (2000) noted: 
Working sensitively and effectively with religious and spiritually oriented clients often requires more than just general multicultural attitudes and skills. It can require specialized knowledge and training about the religious beliefs and practices of religious traditions and communities, about the spiritual issues and needs of human beings, and about religious and spiritual assessment and intervention techniques. (p. 12)

Training programs for clinicians seldom include specific instruction in how to address religious and spiritual issues in counseling, even in the multicultural counseling courses they offer (Shafranske \& Malony, 1990). If faith competence and multicultural competence are distinct competencies, then multiculturally competent counselors are no more or less prone to clinical judgment faith bias than counselors who are not multiculturally competent. The clinical judgments of clinicians high in multicultural competence, then, would not be any different from the clinical judgments of clinicians low in multicultural competence. This prediction has not yet been tested empirically. Multicultural competence is frequently conceptualized not as a single competency but instead as a collection of competencies. For example, in their seminal article on multicultural counseling competencies and standards, Sue, Arredondo, and McDavis (1992) proposed multicultural competence is comprised of three broad dimensions which should be addressed in the training of clinicians: beliefs and attitudes, knowledge, and skills. On the other hand, the Professional Standards Committee of the Association for Multicultural Counseling and Development proposed multicultural competence is comprised of no less than 31 distinct competencies (Sue et al., 1992). One of the most widely-used measures of multicultural competence, the Multicultural Counseling 
Knowledge and Awareness Scale, theorizes (and provides psychometric support for the idea) that multicultural competence consists of two competencies: awareness and knowledge (Ponterotto \& Potere, 2003). Awareness of multicultural issues and knowledge of how to address them when they arise in treatment may influence clinical judgment faith bias in different ways, so examining both of these aspects of multicultural competence is warranted:

Multicultural Awareness: those personal beliefs and attitudes that lead to an awareness of one's own cultural values, the worldviews of others, and appropriate interventions for people from different cultures

Multicultural Knowledge: the specific knowledge and skills that lead to an awareness of one's own cultural values, the worldviews of others, and appropriate interventions for people from different cultures

\section{Clinician Magnitude of Personal Faith and Faith Bias}

A clinician's magnitude of personal faith may also have a moderating effect on any clinical judgment faith bias that exists. Lewis and Lewis (1985) surveyed 77 licensed psychologists involved in clinical work and noted psychologists saw religious patients as needing fewer counseling sessions than nonreligious patients - perhaps seeing religious patients as having better social support, or perhaps viewing them as poorer candidates for long-term counseling than nonreligious patients. The surveyed psychologists also conceptualized religion as having more influence on the presenting concerns of religious patients than on the presenting concerns of nonreligious patients:

Although therapists failed to show evidence of bias in terms of attraction to the patient and in relation to several prognostic variables, they did see the religious 
patient's spiritual orientation as having a large impact on her difficulties. Because the symptoms of depression presented in the religious and nonreligious tapes were virtually identical, it is interesting to note that psychologists found religion to be a very important factor in one case and of relatively little or no importance in the other. (Lewis \& Lewis, 1985, p. 931)

Individual clinicians, however, differ greatly in their willingness to integrate religious issues into counseling (Frame, 2003). Most clinicians report they are willing to discuss religious and spiritual issues in therapy if their clients bring up the issue first (Worthington et al., 1996), but the willingness of clinicians to bring these issues up or incorporate them into counseling appears to be heavily influenced by the religious beliefs of those clinicians. Clinicians who are religious are much more likely to view faith as relevant, important, and helpful to the process of therapy, and more likely to bring it up in counseling, than clinicians who are nonreligious (Kahle, 1998; O'Malley, Gearhart, \& Becker, 1984). Following an extensive meta-analysis of 26 studies of 5,759 therapists and their integration of religious and spiritual issues in counseling, Walker et al. (2004) concluded across multiple studies utilizing different samples and a multitude of therapists from varying backgrounds, the personal religiousness of therapists "was associated with being able to integrate religion and spirituality into several aspects of counseling (e.g., the use of spirituality, being willing to discuss religious issues, even choice of theoretical orientation)" (p. 76).

Studies investigating whether the religiousness of clinicians affects their clinical judgment have yielded mixed results. Gerson et al. (2000) found no relationship between the religious and professional beliefs of therapists, and Wadsworth and Checketts (1980) 
reported the diagnoses 60 Utah psychologists gave to fictional clients was not significantly affected by the religious affiliation of the psychologists. Worthington and Scott (1983) found no evidence secular counselors are insensitive to Christian clients or like religious clients any less (or more) than nonreligious clients. Correspondingly, Gibson and Herron (1990) reported religious and nonreligious therapists do not hold significantly different perceptions of the therapeutic process. By contrast, Kahle (1998) found the willingness of therapists to integrate spirituality into counseling was influenced by the religious beliefs of those therapists, and the aforementioned Lewis and Lewis (1985) study found religious therapists rated both religious and nonreligious patients as more likeable than nonreligious therapists did. In a survey of 199 marriage and family therapists, Hecker, Trepper, Wetchler, and Fontaine (1995) reported highly religious therapists viewed clients as more sexually addicted than therapists low in religiousness. Hillowe (1985) found the religiousness of therapists is negatively correlated with estimates of the number of therapy sessions required by all clients. Likewise, in a survey of 65 mental health professionals and clergy, O’Malley, Gearhart, and Becker (1984) concluded values were more important in the therapeutic process for religious counselors than for nonreligious counselors. The methodologies of these studies varied, but overall, neither the group of studies supporting a link between clinician religiousness and clinical judgment nor the group of studies finding no such link had superior methodologies to the other group. Thus clinical judgments may be affected by clinician religiousness in some cases, but this is not clear.

Perhaps the interaction of clinician and client religiousness influences the judgment of clinicians more than clinician religiousness alone. According to one study, 
the diagnoses 60 Utah psychologists gave to fictional clients were not significantly affected by the interaction between the religion of the psychologist and the religion of the client (Wadsworth \& Checketts, 1980). According to four other studies, though, the interaction of clinician and client religiousness did influence the judgments clinicians made. Hillowe (1985) found the nondoctrinal (i.e., intrinsic) religious attitudes of therapists are positively correlated with estimates of prognosis of religious clients. Gerson et al. (2000) reported a significant negative correlation between the religious beliefs of 87 clinical psychologists and their clinical ratings of a religious case vignette (but not a nonreligious vignette), as well as a significant positive correlation between the strength of psychologists' religious beliefs and optimism for both a religious and a nonreligious case. Similarly, a study of 59 Kentucky psychologists found religious counselors were most optimistic about a religious client, and nonreligious counselors were most optimistic about a religiously doubtful client (Jones, 1991). In a fourth study which was particularly well-designed, Houts and Graham (1986) had 24 religious counselors and 24 nonreligious counselors view videotapes of a nonreligious, moderately religious, or very religious client. Religious counselors made more internal attributions for the nonreligious client, and nonreligious counselors made more internal attributions for the religious client. Interestingly, all of the counselors tended to make the fundamental attribution error (Baron \& Byrne, 1997), attributing the symptoms of clients who held similar beliefs to external, temporary, or circumstantial causes, while attributing the symptoms of clients who held different beliefs to internal, permanent, characterological traits. The results of Houts and Graham's (1986) study, then, suggest clinical judgments of counselors are strongly influenced by the similarity between the 
beliefs of the counselor and client. This fits with Thomas Szasz's (1970) much earlier assertion that the more a patient's values differ from the values of a therapist, the more likely the therapist is to give the patient a severe diagnosis and poor prognosis.

Presumably, clinicians who are religious are more familiar with religious issues than are clinicians who are nonreligious - or at least more familiar with the specific religious issues related to their particular religion. Consequently, religious clinicians are more likely than nonreligious clinicians to be familiar with how those issues impact their clients, including perhaps the relevant base rates of psychopathology among religious persons, and possibly also the research literature demonstrating faith bias in clinical judgment. At the very least, religious clinicians are likely to be aware of the negative stereotype that religiousness is associated with psychopathology, and to recognize this stereotype is in most cases untrue. If this is the case, then clinicians who are religious or spiritual may have faith competence and be less likely to allow the representativeness heuristic, salience bias, and stereotyping to interfere with their clinical decision-making. Religious clinicians - clinicians who have a high magnitude of personal religious faith may be more likely than other clinicians to have some competence in dealing with religious issues, while spiritual clinicians may be more likely to have some competence in dealing with spiritual issues. Accordingly, they may be less susceptible than other clinicians are to clinical judgment faith bias. On the other hand, if religious and spiritual clinicians make the same clinical judgments as other clinicians, this would suggest personal faith alone is not sufficient to confer competence in addressing issues of faith. Clinician Religiousness: the magnitude or level of a clinician's personal religious faith 
Clinician Spirituality: the magnitude or level of a clinician's personal spiritual faith

\section{Clinician View of Faith Helpfulness}

Finally, a clinician's opinion on the helpfulness of faith to the process of counseling may also influence whether clinical judgment faith bias becomes manifest. A clinician's beliefs about how the world works, personal religious practices, and professional attitude towards the role faith should take in clinical practice are three different elements of that clinician's worldview (Beutler \& Bergan, 1991; Hecker et al., 1995; Worthington et al., 1996). A clinician's magnitude of personal faith and his or her view about the helpfulness and relevance of faith to clinical practice are distinct mechanisms which can influence clinical decision-making. As noted previously, psychology is one of the least religious professions, with 50\% of members of psychology departments at universities declaring no religious preference (Shafranske, 1996), yet a majority of psychologists nevertheless view religion as valuable. Fifty-three percent of the respondents in Shafranske and Malony (1990) described religion as valuable, while $65 \%$ agreed spirituality was personally relevant to them. Lannert (1992) found a majority of internship training directors described spirituality as personally relevant to them, and Bergin and Jensen (1990) reported 77\% of all the psychotherapists they surveyed tried hard to live their lives according to their religious beliefs. A large number of therapists, then, must view faith as potentially helpful and relevant to the issues which clients bring in to therapy, in spite of holding relatively low levels of personal religiousness themselves. 
The professional attitudes clinicians hold about mental health and psychopathology are greatly affected by their own personal values, and in therapy, clinicians' values appear to hold more influence than their clients' values. In successful therapy, client and counselor values tend to converge (Worthington et al., 1996), and Hecker et al. (1995) noted "that when client and therapist values converge, the therapist is likely to rate the client as improving, despite the fact that clients may rate themselves as not having improved, or a standardized measure of symptom alleviation does not indicate improvement (Beutler et al., 1983)" (cited in Hecker et al., 1995, pp. 261-262). In other words, the personal values and attitudes of clinicians impact the professional process and outcome of their clinical work, even when clinicians do not intend for this to happen (Beutler \& Bergan, 1991).

Furthermore, decades of attitude research has shown attitudes do not always predict behavior (Cacioppo, Claiborn, Petty, \& Heesacker, 1991; Heesacker, Conner, \& Prichard, 1995). Hillowe (1985) found while therapists' clinical judgments of clients were not significantly related to the therapists' degree of involvement with a religious organization, the therapists' personal religious attitudes notably impacted their professional ratings of the number of sessions required for religious clients to make significant progress in therapy. Notably, the large Walker et al. (2004) meta-analysis described earlier found a moderate relationship between the religious backgrounds of therapists and their use of spiritual and religious issues in therapy but no significant relationship between therapists' religious backgrounds and their willingness to include spiritual and religious issues in therapy. 
Clinicians may have agnostic or atheist personal beliefs and no particular religious practices, yet still hold respect for clients who have strong religious beliefs and think those beliefs have a direct impact on the process of therapeutic change which takes place for those clients (Lewis \& Lewis, 1985). Conversely, clinicians may have strong religious beliefs and a great deal of involvement in religious matters in their own personal lives, yet be reluctant to incorporate these beliefs into their clinical work for fear of imposing their own values on clients. In fact, several studies suggest religious clinicians often feel a sense of internal conflict over the integration of their personal religious and professional beliefs - a conflict which is not typically present in nonreligious clinicians (Eckhardt, Kassinove, \& Edwards, 1992; Kassinove \& Uecke, 1991).

Clinician view of faith helpfulness, then, is a distinct variable from clinician magnitude of personal religiousness and spirituality, although the three variables are closely related. Like religious and spiritual clinicians, clinicians who view faith as being helpful and relevant to the counseling process may also have faith competence and be less likely than other clinicians to allow stereotyping, salience bias, and the representativeness heuristic to interfere with their clinical decision-making. For the same reasons as religious and spiritual clinicians, clinicians who view faith as helpful and relevant to counseling may be less likely than other clinicians to fall victim to clinical judgment faith bias, even if these clinicians are not religious or spiritual themselves. Consequently, because personal religious beliefs and professional beliefs about the helpfulness of religion to counseling are distinct aspects of clinical values, each bears separate empirical examination. 
Clinician View of Faith Helpfulness: the degree to which a clinician perceives religiousness or spirituality to be helpful and relevant to the process of counseling

\section{Summary}

Clinical Judgment Faith Bias refers to a hypothesized tendency for clinicians to make more pathological clinical judgments for clients with higher magnitudes of faith than for otherwise identical clients with socially normative faith. A growing number of research articles on clinical judgment support the contention that clinicians sometimes show bias in the clinical judgments they make of religious clients. Their diagnostic and prognostic judgments are sometimes biased by client religiousness - in fact, they sometimes give different diagnoses to and estimate worse prognoses for highly religious clients than secular clients with identical symptoms (Gartner et al., 1990a; Hillowe, 1985; Houts \& Graham, 1986; Lewis \& Lewis, 1985; O’Connor \& Vandenberg, 2005). These studies suggest religious judgment bias may be more related to the magnitude or social deviance of those beliefs and practices than to their content. Socially deviant forms of religiousness do not just differ from those of the majority but are also considered by society to be strange, weird, or sometimes socially unacceptable. There is no evidence to suggest highly religious people are more likely to experience psychopathology than less religious people (Frame, 2003; Miller \& Kelley, 2005). In fact, some evidence suggests highly religious people may actually have less psychopathology than less religious individuals (Miller \& Kelley, 2005; Oman \& Thoresen, 2005). Yet clinicians do formulate more pathological diagnostic and prognostic judgments for religious clients, especially for clients who are very religious and who present with anxiety or obsessional 
symptoms. These judgment differences presumably represent a clinical bias which should be addressed: a clinical judgment faith bias.

Stereotyping, salience bias, and the representativeness heuristic may help to explain why clinical judgment faith bias might occur. Clinicians may be aware of several stereotypes about religious people, including the false ideas that religion is associated with psychopathology, deviance is related to psychopathology, and religion is linked to obsessional symptoms (Barlow \& Durand, 1995; Miller \& Kelley, 2005; Yossifova \& Loewenthal, 1999). In situations in which high magnitudes of religiousness make the aforementioned stereotypes salient, salience bias and the representativeness heuristic may lead clinicians to ignore base rates and decide these stereotypes are representative of highly religious people (Baron \& Byrne, 1997; Garb, 1998; Kahneman \& Tversky, 1973; Nisbett \& Ross, 1980; Taylor, 2006).

Previous studies addressing this bias have had three primary weaknesses. First, many of them have examined merely the presence or absence of religious ideation or behavior, rather than differing levels of religiousness (e.g., Gartner, Harmatz, Hohmann, Larson, \& Gartner, 1990a, 1990b; Gerson, Allen, Gold, \& Kose, 2000; Hillowe, 1985; Lewis \& Lewis, 1985; Marcinkowski, 1993). Second, those studies examining different magnitudes of religiousness in an effort to examine the social deviance of client faith have either examined different levels of qualitatively different types of religious expressions (e.g., O'Connor \& Vandenberg, 2005) or have investigated forms of faith so socially deviant as to be artificial, inauthentic fabrications of faith not represented in the real world (e.g., Sanderson et al., 1999). Another weakness in many previous investigations of clinical judgment faith bias is their almost exclusive examination of 
client religiousness; virtually no studies have examined the similar construct of client spirituality.

If clinical judgment faith bias does exist, perhaps some clinician individual differences are associated with resilience to this bias, making certain clinicians less likely than others to make biased judgments of highly religious clients. Not all clinicians may be equally susceptible to making biased clinical judgments because of individual differences in the way they process clinical information. Specifically, clinician multicultural awareness and knowledge, magnitude of personal religiousness and spirituality, and view of faith helpfulness may have moderating effects on any clinical judgment faith bias that exists (Kahle, 1998; Lewis \& Lewis, 1985; Worthington et al., 1996). These clinician attributes warrant examination in the interest of identifying individual differences associated with resilience to bias.

The primary purpose of this dissertation, then, is to examine the hypothesized phenomenon of clinical judgment faith bias, to experimentally test if clinicians engage in this bias under certain conditions. This dissertation also tests whether clinician multicultural awareness, multicultural knowledge, personal religiousness, personal spirituality, and view of faith helpfulness are associated with resilience to this bias. Clinical judgment faith bias is a new term, and it refers to a concept on which very little past research has been conducted. The effect on clinical judgment of multicultural competence, clinician faith, client faith, clinician view of faith helpfulness, and the interaction of client and clinician faith have all been researched prior to this study, but no previous research has combined all of these variables into a single study or specified this level of interaction. McCullough and Larson (1998) noted most of the research conducted 
to date in the psychology of religion has been generalist in nature. They pointed out the lack of specialization in the field often "leads to bland generalizations," and "to build a knowledge base, researchers should begin to specialize" (p. 97) by studying specific diagnoses and specific subject variables "in order to develop a broader base of consensually validated and clinically useful knowledge about the relationship between religion and mental health that avoids the bland generalizations that necessarily result from aggregating findings too broadly" (p. 98). The current study attempts to achieve this greater level of specificity by investigating one specific diagnostic group (anxiety symptoms) and five specific clinician subject variables (clinician multicultural awareness, multicultural knowledge, personal religiousness, personal spirituality, and view of faith helpfulness), employing vignettes developed using a rigorous magnitude estimation scaling design and measuring spirituality as distinct from religiousness. The results of this study, then, may be of interest to researchers in the fields of clinical judgment, the psychology of religion, and multicultural counseling alike. It is hoped this study might in some way contribute to a broader base of consensually validated and clinically useful knowledge about the relationship between religion and mental health. 


\section{Chapter 3}

\section{Method}

The purpose of this dissertation is to test for the presence of a clinical judgment faith bias, a hypothesized tendency for clinicians to make more pathological clinical judgments for clients with higher magnitudes of faith than for otherwise identical clients with socially normative forms of faith. Client magnitude of faith is predicted to interact with client type of faith (the Faith Combination hypothesis) such that clinicians not only make increasingly more pathological judgments for cases as the magnitude of religiousness or spirituality portrayed in the cases increases (the Faith Magnitude hypothesis), but this increase in pathology judgments is more pronounced for religious cases than for spiritual cases (the Faith Type hypothesis). Furthermore, the clinician attributes of multicultural awareness, multicultural knowledge, personal religiousness, personal spirituality, and view of faith helpfulness are predicted to moderate the impact of client faith type, client faith magnitude, and their interaction on clinical judgments (the Resilience hypothesis). To test these four hypotheses, a multivariate factorial design was employed. In this chapter, the methods used are reported. The participants, demographics, 
power analysis, dependent and independent measures, case vignette construction, procedures, and research design are reported below.

\section{Participants}

Demographics. The participants in this study were psychologists in clinical practice, members of the American Psychological Association (APA) engaged in parttime or full-time clinical practice. A preliminary power analysis (described below) indicated at least 197 participants would be needed in order to conduct the current study. Anticipating approximately $50 \%$ of solicited psychologists would agree to participate in this study (Dillman, 2007; Heppner, Kivlighan, \& Wampold, 1999), 400 psychologists were randomly selected from APA's membership. Mailing labels for these psychologists were provided by the APA Research Office (APA's internal Institutional Review Board), which was requested to provide a random sample of 400 psychologists currently in clinical practice. A total of 186 of these potential participants responded, either by saying they were declining to participate $(n=12)$, by returning a completed $(n=141)$, partially completed $(n=3)$, or blank survey $(n=6)$, or by returning the return envelope without a survey $(n=24)$, often with a note to the researcher or the dollar incentive the researcher sent out with the first survey mailing. Six of these respondents noted they were not currently in clinical practice, suggesting a much larger proportion of the total sample which was provided by the APA Research Office may not have been practicing clinicians as requested. Of the 186 psychologists who responded, 144 surveys were returned. Three of those surveys had significant amounts of missing data, so they were not analyzed, resulting in a final sample size of 141 and a return rate of 141 out of 400 , or $35.25 \%$. This 
return rate was adequate for an experimental study and typical for a clinical judgment study (c.f. Spengler \& Strohmer, 1994; Walker, 1999).

These participants were randomly selected from APA, so it was expected their demographics would approximate APA's membership. The current sample consisted of 60 males $(44.1 \%), 75$ females $(55.2 \%)$, and 1 person of unspecified gender $(0.7 \%)$ between the ages of 30 and 84 , with an average age of $54(S D=9.2)$. The sample was 91.9\% Caucasian American $(n=125), 0.7 \%$ African American $(n=1), 2.2 \%$ Asian American $(n=3), 2.9 \%$ Hispanic American $(n=4)$, and 2.2\% Other or Not Specified $(n=3)$. Of those psychologists surveyed, 53.7\% had Ph.D.'s $(n=73)$, while $16.2 \%$ had Psy.D.'s $(n=22) ; 24.3 \%$ had unspecified doctorates $(n=33)$. Approximately $36.8 \%$ were clinical psychologists $(n=50), 16.2 \%$ were counseling psychologists $(n=22)$, and $2.2 \%$ were educational or school psychologists $(n=3)$. Similarly, APA member psychologists in the United States as a whole are 51\% male and $49 \%$ female, mostly between the ages of 30 and 65, and 77\% Caucasian American, 2\% African American, 2\% Asian American, 2\% Hispanic American, and 17\% Other or Not Specified. Approximately $80 \%$ have Ph.D.'s, while just over 8\% have Psy.D's. Close to 54\% are clinical psychologists, $11 \%$ are counseling psychologists, and $22 \%$ are affiliated with another specialty area (APA Directory Survey, 1999). Compared to APA member psychologists as a whole, the current sample has a larger age range, includes proportionally more female psychologists and psychologists with Psy.D.'s, and includes proportionally less African American psychologists, psychologists of other ethnic backgrounds, psychologists with Ph.D.'s, and clinical psychologists. The demographic characteristics of the participants in the current 
study are shown in Appendix A. Participants' religious demographics are shown in Appendix B.

The number of nonrespondents, and the number of unusable respondents who did not return usable surveys, suggest problems with attrition. For the 45 unusable respondents (11.3\%) and the 214 nonrespondents (53.5\%), no demographic data was available, apart from gender. The nonrespondents were $48.9 \%$ male $(n=91)$ and $50.5 \%$ female $(n=94)$, comparable to the gender makeup of the respondent sample. On the other hand, the unusable respondent group was $31.1 \%$ male $(n=14)$ and $68.9 \%$ female $(n=31)$, containing a higher proportion of females than the respondent sample. No other comparisons between respondents, nonrespondents, and unusable respondents were possible.

Power analysis. In order to achieve a sample size sufficiently large enough to detect a statistically significant effect, a power analysis was conducted. Statistical power depends primarily on (a) number of participants, (b) alpha, and (c) effect size (Heppner, Kivlighan, \& Wampold, 1999). A power level of .80 is conventionally sought in the social sciences, and experiment-wise alpha is typically set at either the .05 or .01 level (Tabachnick \& Fidell, 2001). For the current study, experiment-wise alpha was set at the .05 level a priori to maximize the chances of detecting a statistically significant difference and to reduce the chances of making a Type II error.

To determine an appropriate anticipated effect size to test the planned hypotheses, a total of nine prior research studies were reviewed because of the relevance of their findings to the current study: Feeser (1997), Gerson, Allen, Gold, and Kose (2000), Gushue, Constantine, and Sciarra (2008), Hecker, Trepper, Wetchler, and Fontaine 
(1995), Hillowe (1985), Marcinkowski (1993), Minyard (1983), O’Connor and Vandenberg (2005), and Sanderson, Vandenberg, and Paese (1999) (see Appendix C). These studies investigated the influence of religious bias or multicultural competence on clinical judgments of pathology - the foundation of clinical judgment faith bias. The attribute-by-treatment hypothesis of greatest interest to the current study is the Resilience hypothesis. Three of these nine studies reported attribute-by-treatment interaction effect sizes: Feeser (1997), Gerson et al. (2000), and Hillowe (1985). For the interaction effect of client/vignette religiousness and clinician religiousness on clinical judgments of pathology, three mean effect sizes were calculated, one from each study: $r=.26$ (Feeser, 1997), $r=.39$ (Gerson et al., 2000), and $r=.13$ (Hillowe, 1985) (see Table 10 in Appendix C). The overall mean of these three effect sizes is $r=.26$, a medium effect size (Cohen, 1988). The current study is a replication of Feeser's (1997) dissertation, in which a stepwise multiple regression analysis was used in an attribute-by-treatment design to investigate the contribution of clinician and client religious and spiritual orientation (and perceived client attractiveness) to overall prognosis. Based on this prior research, the predicted effect size for the current study was set at a medium effect size of $r=.26$ (Cohen, 1988).

The computer program G-Power 3.1.2 (Faul, Erdfelder, Lang, \& Buchner, 2007) was used to calculate the number of participants necessary for the current study. As noted below, five multiple regression analyses were planned, each with two independent client treatment variables (faith magnitude and faith type), one treatment interaction variable (faith magnitude $x$ faith type), one independent clinician attribute variable (multicultural awareness, multicultural knowledge, religiousness, spirituality, or view of faith 
helpfulness), two 2-way attribute-by-treatment interaction variables (the clinician attribute $x$ faith magnitude, and the clinician attribute $x$ faith type), and one 3-way attribute-by-treatment interaction variable (the clinician attribute $\mathrm{x}$ faith magnitude $\mathrm{x}$ faith type). To maximize statistical power, experiment-wise alpha was set at a standard .05 , but because five analyses were planned, experiment-wise alpha was divided by five, using a Bonferroni correction to control for inflated Type II error (Tabachnick \& Fidell, 2001). This resulted in a test-wise alpha of .01 for each regression. G-Power calculates that for a regression with 2 tested predictors (the manipulated independent variables) and a total of 7 predictors (including interaction terms), a minimum of 197 participants are necessary to detect an attribute-by-treatment effect size of $r=.26\left(f^{2}=.07\right)$ at a .01 test-wise alpha level to achieve a conventional power level of .80. The current study, however, only obtained 141 participants. G-Power calculates that if each planned regression obtains attribute-by-treatment effect sizes of no larger than $r=.26\left(f^{2}=.07\right)$, it will only obtain a power of .61 at an alpha of .01, indicating that the planned regressions have somewhat low power to detect an effect size of the anticipated magnitude (Cohen, 1988).

\section{Case Vignette}

Six versions of a case vignette were created to serve as the treatment condition in the current study (see Appendix D1 and Appendix F). This vignette describes a fictional client named Mary who has Generalized Anxiety Disorder (GAD) and allows two independent variables to be manipulated: client faith type (religiousness or spirituality) and client faith magnitude (high, moderate, or low). The first paragraph of the vignette gives basic demographic information about Mary and describes her symptoms of GAD. To control for extraneous variables and ensure participants responded to these vignettes 
only on the basis of the religiousness or spirituality they portrayed, all versions of the vignette included an identical first paragraph. The second paragraph of the vignette describes Mary's magnitude or level of faith and represents the two treatments or experimental manipulations conducted in the current study, faith type and faith magnitude. Three versions depict a client with high, moderate, or low religiousness, while three versions depict a client with high, moderate, or low spirituality. The procedure which was used to develop this vignette is described here briefly and depicted in more detail in Appendix G.

This vignette was developed based on religious and spiritual vignettes from Hillowe (1985) and Feeser (1997), Minyard's (1983) religious cues, and items from several measures of religiousness and spirituality, including the Religious Values Scale (Morrow, Worthington, \& McCullough, 1993), the Religious Orientation Scale (Allport \& Ross, 1967; Gorsuch \& McPherson, 1989), the Religious Belief Inventory (Lee, 1965), the Attitude Toward Evangelism Scale (Seyfarth, Larsen, Lamont, Haasch, Hale, \& Haskin, 1984), the Omnibus Personality Inventory - Religious Orientation Scale (Heist \& Yonge, 1968), the Index of Core Spiritual Experiences (Kass, Friedman, Lesserman, Zuttermeister, \& Benson, 1991), the Mysticism Scale (Hood, 1975), the Spiritual Assessment Inventory (Hall \& Edwards, 1996), and the Spiritual Well-Being Questionnaire (Moberg, 1984). The six different versions of this vignette were developed and validated in such a way that the high religiousness and spirituality versions portray a person who is approximately twice as religious or spiritual as the moderate religiousness and spirituality versions, which in turn portray a person who is roughly twice as religious or spiritual as the low religiousness and spirituality versions. 
These vignette versions were developed over the course of three pilot studies. The first pilot study was a manipulation check conducted to ensure the first paragraph of the vignette accurately portrays a client presenting with GAD. Fourteen psychologists in clinical practice rated on a 7-point Likert-type scale how accurately this paragraph depicts a person whose presenting concern is Generalized Anxiety Disorder. The questionnaire given to these psychologists appears in Appendix H. These 14 psychologists rated GAD as the most accurate diagnosis, giving it an average accuracy rating of 6.64 - more than twice the accuracy rating of the diagnosis rated next most accurate $(\mathrm{OCD}=2.93)$. The data from this pilot study are shown in Table 11 in Appendix G.

The second pilot study developed two pools of potential vignette versions and content-validated them, based on a procedure outlined in Lodge (1981) and Spengler, Blustein, and Strohmer (1990). A pool of 16 religious versions and a second pool of 21 spiritual versions were written, each containing six sentences in which six different qualities are systematically varied from high to low: (1) a cognitive statement of faith, (2) a personal statement of faith, (3) a public expression of faith, (4) a private expression of faith, (5) charitable involvement, and (6) evangelism/openness. The 16 religious vignette versions were written to portray a member of a general, nondenominational form of Protestant Christianity. Vignette versions were generated to reduce the variability in their religious content and to represent an ecologically valid, real-world authentic form of religious expression representing a wide variety of beliefs and practices ranging from high to low religiousness and from conservative to liberal. The vignette versions were written in an attempt to faithfully portray a specific Christian denomination: Baptist 
Christianity, the largest and most diverse Christian denomination in the United States (excluding Catholicism, which has specific beliefs and practices setting it apart from the majority of other Christian denominations). The 21 spiritual vignette versions were generated by first reviewing recent research on the similarities and differences between the constructs of religiousness and spirituality (e.g., Cook, Borman, Moore, \& Kunkel, 2000; Hill, Pargament, Hood, McCullough, Swyers, Larson, \& Zinnbauer, 2000; Zinnbauer \& Pargament, 2005) and then rewriting the religious vignette versions to reflect spiritual content while maintaining the same six qualities of the original versions. The 16 religious and 21 spiritual vignette versions are presented in Appendix I.

To evaluate the content validity of the two vignette pools, they were first reviewed, edited, and revised by a clinical judgment expert and a psychology of religion expert to more accurately represent varying levels of religiousness and spirituality. The revised vignette versions were then submitted to a pool of 11 expert raters in Muncie, Indiana and Miami, Florida (3 Baptist ministers, 7 religious studies professors, and 1 Unitarian Universalist pastor), who rated the extent to which each vignette accurately represents an authentic expression of religiousness and spirituality. The questionnaire given to these religious expert raters appears in Appendix $\mathrm{J}$, and their mean accuracy ratings are displayed in Table 12 in Appendix G. The 13 religious vignette versions and 13 spiritual vignette versions with the highest mean accuracy ratings were selected for further use.

In the third pilot study, the level of religiousness or spirituality in the vignette versions was measured and six vignette versions were selected for the current study, using magnitude estimation scaling (Lodge, 1981) based on Tanenhaus and Murphy 
(1981) and Spengler, Blustein, and Strohmer (1990). The vignette versions with the highest accuracy ratings from the second pilot study were pilot-tested with undergraduate student volunteers, who rated the degree of religiousness or spirituality represented by each vignette. The questionnaires given to these students appear in Appendix K. First, 23 undergraduate students completed a cross modality matching task, which served as a mathematical correction for the regression bias which typically occurs in the magnitude estimation scaling of social stimuli. The students were given a labeled $50 \mathrm{~mm}$ reference line and were asked to estimate how long 12 given lines were, to assign number estimates to those lines, and then to draw lines to match the length of 12 numbers provided. Second, students were given a reference vignette describing a moderately religious client, which was paired with an assigned number of 50 and a $50 \mathrm{~mm}$ reference line. Students were then given 13 of the religious vignette versions with the highest accuracy ratings and asked first to assign a number estimate and then to draw a line representing the magnitude of religiousness described by each vignette version. Similarly, a separate group of 23 students were given 13 of the spiritual vignette versions with the highest accuracy ratings and asked to assign number estimates and draw lines for these vignette versions. Afterwards, magnitude estimation scale values were calculated for the levels of religiousness and spirituality of the vignette versions by determining the geometric means for both the number estimates and the length of the lines participants drew in response to the vignette versions. These geometric means were then corrected for regression bias by raising each one by the inverse of the slope obtained by calculating the geometric means for both the number estimates and the length of the lines participants initially drew in response to the given lines and numbers. Finally, a magnitude estimation scale value was 
calculated for each vignette version by computing the square root of the product of the adjusted number-estimate and line-length means. The results of these calculations are displayed in Table 13 in Appendix G.

Following these mathematical procedures, six vignette versions were selected to represent high, moderate, and low religiousness and spirituality, based on their magnitude estimation scale values and uncorrected mean magnitude scores. These vignette versions, their mean magnitude scores, and their magnitude estimation scale values are shown in Table 1. This procedure enabled client faith magnitude - both religiousness and spirituality - to be treated as continuous ratio variables, using magnitude estimation scaling to provide a ratio index of the relative strength of faith in the six vignette versions.

Table 1

Magnitude Estimation Scaling Calculations

\begin{tabular}{llll} 
Variable & Vignette & Geometric & Magnitude Estimation \\
& Version & Means & Scaling Values \\
\hline High Religiousness & 2 & 129 & 2.08 \\
Moderate Religiousness & 9 & 59 & 1.68 \\
Low Religiousness & 11 & 27 & 1.30 \\
High Spirituality & 18 & 128 & 2.08 \\
Moderate Spirituality & 26 & 53 & 1.70 \\
Low Spirituality & 31 & 24 & 1.26
\end{tabular}




\section{Measures}

The Multicultural Counseling Knowledge and Awareness Scale (MCKAS). The Multicultural Counseling Knowledge and Awareness Scale (MCKAS) is a 32-item, self-report measure of ethnocentrism and general multicultural counseling knowledge which was selected to measure the independent variables of clinician multicultural knowledge and multicultural awareness (Ponterotto, Gretchen, Utsey, Rieger, \& Austin, 2002; see Appendix D3). The MCKAS is based on the multicultural counseling competencies model by Sue and colleagues (Sue, Bernier, Durran, Feinberg, Pederson, Smith, \& Vasquez-Nuttall, 1982; Sue, Arredondo, \& McDavis, 1992) - a model proposing that multicultural competencies are related to the beliefs and attitudes, knowledge, and skills counselors possess which lead them to an awareness of their own cultural values and biases, an awareness of the worldview of others who are culturally different, and a working knowledge of culturally appropriate intervention strategies. Items are measured on a 7-point Likert-type scale ranging from $1=$ Not At All True to 7 $=$ Totally True and ask participants to rate the truth of statements such as "I believe all clients should maintain direct eye contact during counseling," "I am comfortable with differences that exist between me and my clients in terms of race and beliefs," "I understand the impact and operations of oppression and the racist concepts that have permeated the mental health professions," and "I feel all the recent attention directed toward multicultural issues in counseling is overdone and not really warranted." The MCKAS has two subscales: a 20-item Knowledge subscale and a 12-item Awareness subscale. Possible scores for the MCKAS subscales range from 1 to 7 , as subscale scores are obtained by calculating the average of each scale's items (Constantine \& Ladany, 
2001; Ponterotto, Gretchen, Utsey, Rieger, \& Austin, 2002; Ponterotto, Rieger, Barrett, Sparks, Sanchez, \& Magids, 1996; Ponterotto, Sanchez, \& Magids, 1991).

Test-retest reliability coefficients for the Knowledge subscale (.70) and the Awareness subscale (.73) are satisfactory (Manese, Wu, \& Nepomuceno, 2001; Ponterotto \& Potere, 2003). Coefficient alphas range from .75 to .93 for the total scale, from .78 to .93 for the Knowledge subscale, and from .72 to .89 for the Awareness subscale, reflecting good internal consistency. Multicultural expert ratings of item clarity and appropriateness support the content validity of the scale (Ponterotto et al., 1996). Initial principal components factor analyses with oblique rotations using one-, two-, three-, and four-factor extractions by Ponterotto et al. (1996) and later confirmatory factor analyses by Ponterotto et al. (2000, 2002) supported a two factor extraction which had better goodness-of-fit index scores $(\mathrm{GFI}=.90, \mathrm{TLI}=.91, \mathrm{RNI}=.93)$ than competing models and accounted for between $28 \%$ and $32.2 \%$ of common variance. Ponterotto and Potere (2003) reviewed 19 studies examining the psychometric properties of the MCKAS, and concluded the instrument's two subscales have reasonable evidence for convergent, discriminant, and criterion-related validity. Low to moderate correlations between the subscales (generally ranging from .22 to .50 ) are consistent with Sue and colleagues' (1992) hypothesis that the awareness and knowledge components of multicultural competence are only moderately related and should be separately analyzed in research studies (Ponterotto \& Potere, 2003).

The MCKAS has two notable limitations. The scale is prone to ceiling effects when administered to experienced clinicians (Constantine \& Ladany, 2000; Ponterotto et al., 1996, 2000, 2002; Ponterotto \& Potere, 2003). Because clinicians tend to gain a 
moderate to high degree of multicultural competence as a result of their multicultural training, they tend to achieve relatively high scores on the MCKAS, which tends to restrict the range and the variability of obtained MCKAS scores. Second, little extant research exists demonstrating a correlation between MCKAS scores and success in providing counseling services to clients from diverse cultural backgrounds (Constantine \& Ladany, 2000; Ponterotto \& Potere, 2003). Therefore, Ponterotto and Potere (2003) suggest this scale is only appropriate to use for the purposes of group research and should not be used in clinical decision-making. The current study is for research purposes, and alternative methods of assessing multicultural competence which are more highly correlated with actual counseling success (e.g., portfolio reviews) are impractical to implement for mail survey research. Compared to the other most commonly-used measures, the MCKAS is the least susceptible to social desirability bias (Constantine, Gloria, \& Ladany, 2002; Constantine \& Ladany, 2000; Kocarek, Talbot, Batka, \& Anderson, 2001). It is also brief, easy to use, and has good psychometric properties (Ponterotto \& Potere, 2003). For these reasons, the MCKAS was selected for use in the current study to assess clinicians' multicultural competence. Since Ponterotto and Potere's (2003) research supports the independence of the MCKAS Awareness subscale from the MCKAS Knowledge subscale, these two subscales were analyzed as two distinct continuous independent variables: clinician multicultural awareness and multicultural knowledge. The means $(M)$, standard deviations $(S D)$, and Cronbach's alphas $(\alpha)$ for the MCKAS-A and MCKAS-K subscales and all other scales which were administered in the current study are shown in Table 2. 
Table 2

Means (M), Standard Deviations (SD), and Cronbach's Alphas ( $\alpha$ ) for All Scales

Completed by Participants

\begin{tabular}{llccc} 
Variable & Scale & $M$ & $S D$ & $\alpha$ \\
\hline Prognosis & STPE-P2 & 12.00 & 1.80 & .77 \\
Diagnosis & STPE-D & 5.70 & 1.33 & - - \\
Multicultural Awareness & MCKAS-A & 5.72 & .60 & .75 \\
Multicultural Knowledge & MCKAS-K & 5.02 & .78 & .88 \\
Spirituality & S-ROS-I & 4.90 & 1.26 & .86 \\
Religiousness & S-ROS-I & 4.52 & 1.35 & .87 \\
View of Faith Helpfulness & RITSF-Help & 22.17 & 3.35 & .72 \\
\hline
\end{tabular}

Note: The scales completed by participants were the Schedule of Therapists' Prognostic Expectations - (Second) Prognosis (STPE-P2) and Diagnosis (STPE-D) subscales, the Multicultural Counseling Knowledge and Awareness Scale - Awareness (MCKAS-A) and Knowledge (MCKAS-K) subscales, the Spiritual - Religious Orientation Scale Spiritual Intrinsic $\left(\mathrm{S}-\mathrm{ROS}-\mathrm{I}_{\mathrm{S}}\right)$ and Religious Intrinsic $\left(\mathrm{S}-\mathrm{ROS}-\mathrm{I}_{\mathrm{R}}\right)$ subscales, and the Religious Issues in Therapy Survey Form - Help subscale (RITSF-Help). 
The Spiritual - Religious Orientation Scale (S-ROS). The Spiritual - Religious Orientation Scale (S-ROS) is a 28-item measure of the magnitude of a person's faith, using both religious and spiritual language, which was selected to measure the independent variables of clinician religiousness and clinician spirituality (Peterson, 2001; see Appendix D4). Items are measured on a 7-point Likert-type scale ranging from $1=$ Strongly Disagree to $7=$ Strongly Agree (with a N/A response option) and ask participants to rate their level of agreement with statements such as "I enjoy reading books that deepen my understanding of life," "The primary purpose of following spiritual guidance is to avoid personal suffering," and "Quite often I have been keenly aware of the presence of God or the Divine Being." The S-ROS has five subscales: a 9-item Religious Intrinsic subscale $\left(\mathrm{I}_{\mathrm{R}}\right)$, a 3-item Religious Extrinsic - Personal subscale $\left(\mathrm{Ep}_{\mathrm{R}}\right)$, a 3-item Religious Extrinsic - Social subscale $\left(\mathrm{Es}_{\mathrm{R}}\right)$, an 8-item Spiritual Intrinsic subscale $\left(\mathrm{I}_{\mathrm{S}}\right)$, and a 5-item Spiritual Extrinsic - Personal subscale $\left(\mathrm{Ep}_{\mathrm{S}}\right)$. Possible scores for the SROS subscales range from 1 to 7 , as subscale scores are obtained by calculating the average of each scale's items (Peterson, 2001).

The S-ROS is a version of Allport and Ross's (1967) classic Religious Orientation Scale (ROS), modified by Peterson (2001) to include a "spiritually" worded version of the ROS in which spiritual language was substituted for religious language in each item, while still including the classic ROS. Peterson (2001) noted the ROS may not be appropriate for people who are from non-Jewish or non-Christian backgrounds, and even for some nontraditional Christians, so he created an alternative scale which could be used more broadly with people from several different religious backgrounds as well as with people who identify as spiritual but not religious. 
Peterson's (2001) research indicated the S-ROS has acceptable reliability for five of its six subscales. Of the six potential subscales on the S-ROS, five were found through principal components analyses with varimax and oblique rotations. The reliability estimates for the $I_{R}, E p_{R}$, and $E_{s_{R}}$ scales from the original ROS were 0.83, 0.57, and 0.58, respectfully (Gorsuch \& McPherson, 1989). The reliability estimate for the $\mathrm{I}_{\mathrm{S}}$ scale was 0.82, while the reliability estimate for the $\mathrm{Ep}_{\mathrm{S}}$ scale was 0.79 (Peterson, 2001). These reliability estimates are comparable to or better than the reliabilities of the original ROS (Burris, 1999; Genia, 1993, 1994). The S-ROS has relatively high correlations between individual items and the overall subscales they comprised and low correlations among the five subscales. The $I_{R}$ has reasonable evidence to support its content, construct, and criterion-related validity, with relatively high correlations with measures of religious motivation, religious commitment, sense of meaning in life, and social desirability but low correlations with measures of religious maturity and prejudice (Burris, 1999). The $\mathrm{Ep}_{\mathrm{R}}$ and $\mathrm{Es}_{\mathrm{R}}$ correlate highly with measures of status-seeking, comfort-seeking, and religion as being tangential, as well as with measures of mental health, stress, worry, maladjustment, and guilt. They also correlate negatively with measures of religious commitment, as would be predicted for a purely utilitarian motivation which is both externally motivated and distinct from intrinsic religiousness (Burris, 1999). The $\mathrm{I}_{\mathrm{S}}$ scale had a correlation of 0.63 with the $I_{R}$ scale, while the E $p_{S}$ scale had a correlation of 0.72 with the $\mathrm{Ep}_{\mathrm{R}}$ scale. The Es $\mathrm{S}_{\mathrm{S}}$ scale had an alpha of 0.19 ; its internal consistency was deemed too low for analytical work, so this scale was dropped. The correlation of the original religiously worded statements from the ROS to the overall ROS scale ranged from 0.34 to -0.69 , which was generally in the same range found in other studies (e.g., 
Genia, 1993; Kirkpatrick, 1989). The item-to-item correlations of each spiritually worded item and its original religiously worded counterpart ranged from 0.25 to 0.63 and became progressively stronger as the respondent group became closer to traditional samples. There was a high correlation between the "spiritually" worded IS and Eps subscales and their corresponding ROS scales $\left(\mathrm{I}_{\mathrm{S}}\right.$-to- $\mathrm{I}_{\mathrm{R}} r=0.63, p<.001 ; \mathrm{Ep}_{\mathrm{S}}$-to-Ep $\mathrm{R}_{\mathrm{R}} r=0.72, p<$ .001). $I_{S}$ and $E p_{S}$ have similar correlations to measures of prejudice as the $I_{R}$ and $E p_{R}$ have, providing support for the concurrent validity of the S-ROS. "Most importantly for the purposes of this study, a valid $\mathrm{I}_{\mathrm{S}}$ could be calculated for $80 \%$ of the study respondents while the $\left[I_{R}\right]$ was only valid for $68 \%$ of [all] respondents" (Peterson, 2001, p. 76) - and for only $41 \%$ of participants who identified as spiritual but not religious. This not only supports the discriminant validity of the S-ROS, but also demonstrates its utility with a sample of psychologists, who tend to be significantly less religious than the general population (Bergin, 1991).

A 55-item S-ROS was used here to replicate Peterson (2001) and to confirm the factor structure of the S-ROS with another sample. An exploratory factor analysis was conducted using principal components analysis with Direct Oblimin rotation. A KMO value of .66 suggested the data were suitable for analysis $(\mathrm{KMO}=.66)$, and sphericity was not a problem for the current sample (Bartlett's $\chi^{2}=1927.34, d f=861, p<.001$ ) (Gorsuch, 1983). The structure matrix is reproduced in Appendix L1, and the inter-item correlation matrix is reproduced in Appendix L2. The first factor extracted was nearly identical to the spiritual-intrinsic factor identified by Peterson (2001). Inter-item correlations among this factor's 8 items ranged from .16 to .69, while Cronbach's alpha for this factor was .86. The current sample confirmed the internal consistency and factor 


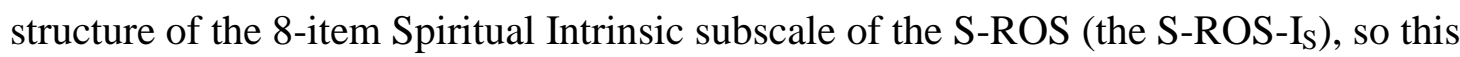
subscale was selected to measure the independent variable of clinician personal spirituality. The Religious Intrinsic subscale of the S-ROS (the S-ROS- $\mathrm{I}_{\mathrm{R}}$ ) was selected to measure the independent variable of clinician personal religiousness. A Cronbach's alpha of .87 was calculated for this subscale, which was consistent with past reported statistics for its internal consistency. The means $(M)$, standard deviations $(S D)$, and Cronbach's alphas $(\alpha)$ for the S-ROS-I $I_{R}$ and $S-R O S-I_{S}$ are shown in Table 2.

Religious Issues in Therapy Survey Form (RITSF). The Religious Issues in Therapy Survey Form (RITSF) is a 19-item, self-report measure of clinicians' attitudes about religious issues in therapy which was selected to measure the independent variable of clinician view of faith helpfulness (Kivley, 1986; see Appendix D5). Items are measured on a 5-point Likert-type scale ranging from $1=$ Strongly Disagree to $5=$ Strongly Agree, asking participants to rate their level of agreement with statements such as "Religious belief is too subjective to be useful in therapy," "Religious activities effectively change one's perceptions of circumstances," and "Religious belief is very relevant to the therapy process." The RITSF is composed of three scales (View of Religious Belief, Utility of Religious Belief, and Therapist Attitude Toward Including Religious Issues in Therapy) as well as five single item variables. Subscale scores are obtained by summing the scores of each subscale's items, while the RITSF total scale score is obtained by summing the scores of all 19 items.

Little psychometric data were reported for the RITSF, as Kivley's (1986) article appears to describe the only published study which has used the RITSF to date. It appears to have adequate internal consistency reliability on its three subscales as measured by 
coefficient alpha: $0.77,0.54$, and 0.68 for the View of Religious Belief, Utility of Religious Belief, and Therapist Attitude Toward Including Religious Issues in Therapy subscales, respectively. No test-retest reliability data were presented. Kivley and two advanced graduate students in the Counseling Psychology program of a large midwestern university judged the RITSF to have content validity, and Kivley (1986) invited the readers of his article to judge the content validity of the RITSF for themselves by publishing the items in the text of his article. No further validity data were presented on this scale. The RITSF appeared to be well-constructed, however, and a search of the peerreviewed literature turned up no other measures of clinician view of faith helpfulness. Consequently, the RITSF was selected for use in the current study to measure the continuous independent variable of clinician view of faith helpfulness.

An exploratory factor analysis was conducted on the 19-item RITSF using principal components analysis with Direct Oblimin rotation because the potential factors were anticipated to be related (Kivley, 1986). The data were found to be suitable for factor analysis $\left(\mathrm{KMO}=.810\right.$, Bartlett's $\left.\chi^{2}=880.588, d f=171, p<.001\right)$. The structure matrix is reproduced in Appendix L3, and the inter-item correlation matrix is reproduced in Appendix L4. Five components were extracted with eigenvalues greater than 1. Correlations among the factors were low, ranging from .03 to .38 . The three factors which Kivley (1986) attempted to construct (View of Religious Belief - items 1-5; Utility of Religious Belief - items 7 and 9-12, and Therapist Attitude Toward Including Religious Issues in Therapy - items 14-17) were not supported. Instead, five factors emerged: a Religion and Self factor consisting of items 1-3, 6, 11-12, and 19; a Religion's Impact on Change factor consisting of items 4, 5, and 7; a Helpfulness of 
Religion in Therapy factor consisting of items 8-10 and 13-15; a Religious Competence factor consisting of items 16 and 17; and a Desire to Discuss Religion factor consisting of item 18. Three follow-up analyses (a principal components analysis with Varimax rotation, a principal axis factoring with Oblimin rotation, and a maximum likelihood analysis with Oblimin rotation) evidenced little difference in factor structure. Because the Helpfulness of Religion in Therapy factor was conceptually most similar to the "View of Faith Helpfulness" characteristic the RITSF was chosen to measure, items 8-10 and 1315 were selected and designated as a new RITSF-Help subscale to measure the independent variable of clinician view of faith helpfulness. Inter-item correlations ranged from .20 to .45 , while Cronbach's alpha for this factor was .74, suggesting this new scale's internal consistency is adequate and represents a slight improvement from the internal consistencies of Kivley's (1986) original scales.

The RITSF-Help subscale, then, is a 6-item subscale consisting of items 8-10 and 13-15 from the RITSF. It is based on factor analysis of the RITSF and consists of items representing how helpful clinicians view religiousness to be in the process of therapy, asking participants to rate their level of agreement with statements such as "Religious belief is too subjective to be useful in therapy," "Religious belief is very relevant to the therapy process," "Helping clients strengthen spiritual expressions is a legitimate goal for therapy," and "Therapists who introduce religious issues into therapy are acting unethically." Possible scores for the RITSF-Help subscale range from 6 to 30. The mean $(M)$, standard deviation $(S D)$, and Cronbach's alpha $(\alpha)$ for the RITSF-Help are shown in Table 2. 
The Schedule of Therapists' Prognostic Expectations (STPE). The current study has two dependent variables of interest: clinicians' diagnostic judgments and clinicians' prognostic judgments. The Schedule of Therapists' Prognostic Expectations (STPE), as modified and used in the current study, is an 18-item, self-report measure of clinicians' prognostic and diagnostic judgments of a client portrayed in a clinical vignette (Graham, 1980; see Appendix D2). The STPE was originally a 9-item scale developed by Graham (1980) and used later by Lewis and Lewis (1985), Hillowe (1985), and Feeser (1997); however, 9 additional items were added here to create a continuous measure of diagnostic judgments. Items 1-6 ask clinicians to rate on 7-point Likert-type scales the client's appropriateness for therapy, selection for the clinician's caseload, severity of impairment, motivation to change, capacity for insight, and likelihood for making substantial progress in therapy. Item 7 asks clinicians to indicate a multiaxial DSM-IV diagnosis, and items 8 and 9 ask clinicians to estimate the number of therapy sessions needed for the client to make substantial progress and the number of sessions the client would actually attend. Items 10-18 ask clinicians to rate on 7-point Likert-type scales ranging from $1=$ Not Very Likely to $7=$ Very Likely the likelihood that the client has Generalized Anxiety Disorder (the correct diagnosis) and eight other anxiety disorders. The modified STPE used here consists of three subscales: a Diagnosis subscale and two Prognosis subscales.

The Diagnosis subscale (STPE-D) consists of item 13, which asks clinicians to rate on a 7-point Likert-type scale the likelihood that the client has Generalized Anxiety Disorder. A clinician's score on this item was used to represent this clinician's diagnosis rating. Items 10-12 and 14-18 are distracter or "filler" items asking clinicians to rate eight 
other incorrect diagnoses on the same 7-point Likert-type scale. Possible scores for the STPE-Diag subscale range from 1 to 7 . The higher the score, the more certain a clinician is about this diagnosis.

The first Prognosis subscale (STPE-P1) consists of items 4, 5, and 6, which ask clinicians to rate on 7-point Likert-type scales "How motivated is the client to change?", "What is the client's capacity for psychological insight?", and "How likely is it that the client will make substantial progress in psychotherapy?" The sum of a clinician's scores on these items was used to represent this clinician's ratings of overall prognosis. Possible scores for the STPE-Prog range from 3 to 21 . The higher the score, the better the rating of prognosis.

The second Prognosis subscale (STPE-P2) consists of items 1 and 2, which ask clinicians to rate on 7-point Likert-type scales "How appropriate a candidate for psychotherapy is this client?" and "Would you choose to work with this client?" The sum of a clinician's scores on these items was also used to represent this clinician's ratings of overall prognosis. Possible scores for the STPE-P2 range from 2 to 14 . The higher the score, the better the rating of prognosis.

Graham (1980) and Lewis and Lewis (1985) both treated each item on the STPE as a separate dependent variable. To determine whether the scale could be treated as a single unitary measure, Hillowe (1985) performed a correlational analysis and a factor analysis of the STPE. Only items 4, 5, and 6 loaded as a single factor for both the Religious and Nonreligious conditions. These items had high positive correlations among them $(r=.42$ to $.71, p=.0001)$. Hillowe (1985) concluded these items measured a single factor, which he called overall prognosis. Subsequently, he analyzed these three items 
together as a single dependent variable, while continuing to analyze the other items individually as separate dependent variables. Though Feeser (1997) gave the entire STPE to her participants, she analyzed only items 4,5 , and 6 (the overall prognosis variable) as a single dependent variable, based on Hillowe (1985).

Unexpectedly, a printing error led to the anchors for question \#1 ("Inappropriate" to "Highly Appropriate") being incorrectly reprinted for items 2-6 instead of the correct anchors (which are reprinted in Appendix D2). As a result, participants' responses for items 4, 5, and 6 were suspect, and their responses for item 3 (which was reverse scored) were invalid. As for item 2, the incorrectly printed anchors for this item matched the wording of the question, and two graduate students independently judged that the printing error likely did not appreciably change the substantive interpretation of clinicians' responses to this question. To determine how the printing error affected the construct validity and reliability of the instrument, the factor structure and inter-item correlations of STPE items in the current sample were compared to those statistics reported in earlier research on the instrument.

An exploratory factor analysis was conducted on the 18 items of the STPE (using the GAF for item 7) using principal components analysis with Direct Oblimin rotation because the potential factors were anticipated to be related (Feeser, 1997). A KMO value of .63 indicated the data were suitable for factor analysis, and a statistically significant Bartlett's value of $\chi^{2}=592.83, d f=153, p<.001$ indicated sphericity was not a problem, so an exploratory factor analysis was performed. The structure matrix is reproduced in Appendix L5. Six components were extracted with eigenvalues greater than 1, suggesting six factors: a prognosis factor consisting of items 4,5 , and 6 (identical to the overall 
prognosis factor found by Hillowe, 1985), a second prognosis factor consisting of items 1 and 2, a diagnosis factor consisting of item 13, a distracter items factor consisting of items 10-11 and 14-18, a sessions factor consisting of items 8 and 9, and a miscellaneous factor consisting of items 3,7 , and 12 . The inter-item correlation matrix is reproduced in Appendix L6. Items 1 and 2 had a sufficiently high between-item correlation $(r=.65)$, forming a second prognosis factor with a respectably high Cronbach's alpha of .77. Because the aforementioned printing error called into question the validity of the first prognosis factor consisting of items 4,5 , and 6 , the second prognosis factor was selected as a more valid measure of clinician prognostic judgments, as it was not appreciably affected by the printing error. Consequently, items 1 and 2 were analyzed as a Prognosis subscale - the STPE-P2 subscale - and used to measure clinician prognostic judgments as a continuous variable. Item 13 was analyzed as a single item diagnosis subscale - the STPE-D - and used to measure clinician diagnostic judgments as a continuous variable. The means $(M)$, standard deviations $(S D)$, and Cronbach's alphas $(\alpha)$ for the STPE-P2 and STPE-D subscales are shown in Table 2.

\section{Procedure}

Dillman's (2007) Tailored Design method for mail and internet surveys, which is based on social exchange theory, was used for the design of the experimental survey and sampling procedure. First, participants were sent by first class mail a prenotice letter informing them about the study and asking them to participate (see Appendix E1). Approximately one week later, participants were mailed a survey booklet containing the Multicultural Counseling Knowledge and Awareness Scale (MCKAS), the Schedule for Therapists Prognostic Expectations (STPE), the Spiritual - Religious Orientation Scale 
(S-ROS), the Religious Issues in Therapy Survey Form (RITSF), demographic questions, and a randomly assigned version of the clinical vignette (see Appendix D). Each survey booklet was accompanied by a detailed cover letter (see Appendix E2), an online address to a website containing debriefing information for the study (see Appendix D7), an addressed, stamped return envelope, and a token financial incentive of \$1. Dillman (2007) found in his research a "thank you" incentive sent with the survey yielded consistently higher survey response rates than lotteries, contributions to charity, offers of prizes, or even much larger payments sent to participants after returning their surveys, because it was viewed as a sign of consideration and respect. Dillman (2007) also found sending surveys by first class mail and including return envelopes with real stamps increased response rates. These procedures were followed in an attempt to increase participation. Approximately one week later, participants were mailed a thank you letter thanking them for participating in the study and reminding them to return their questionnaire packet (see Appendix E3). Four weeks after the questionnaire packet mailing, a replacement questionnaire was sent to all participants who had not yet responded in a nonrespondent mailing (see Appendix E4). Finally, four weeks after the replacement questionnaire mailing, all participants who had not yet responded were mailed a priority mail special contact letter to ask for their participation (see Appendix E5). Again, Dillman (2007) found a 5-step participant contact procedure consisting of a prenotice letter, questionnaire, thank you mailing, replacement questionnaire, and nonrespondent follow-up increased response rates, so these procedures were followed to increase participation. 


\section{Research Design}

To test the hypotheses proposed for investigation, this study employed a $3 \times 2 \times 1$ randomized attribute-by-treatment multivariate fractional factorial experimental design (Heppner, Kivlighan, \& Wampold, 1999). In a factorial design, two or more independent variables interact to exert an effect on one or more dependent variables (Heppner et al., 1999; Krathwohl, 1998). It was a fractional factorial design because not all possible interactions were included - only key interactions of theoretical interest (Hill \& Lewicki, 2007). It was an attribute-by-treatment factorial design because five clinician subject variables (attributes) were examined for their interaction with the independent variables of client faith magnitude and faith type, which were experimentally manipulated in the vignette versions clinicians received (the treatment) (Heppner et al., 1999). Five analyses were conducted, one for each clinician attribute. The study employed a 3 x 2 x 1 design because the independent variables were client faith magnitude (low, moderate, and high), client faith type (religious and spiritual), and one of five clinician attributes (measured as a continuous variable). Two manipulated independent variables represented the treatment under investigation here (client faith magnitude and type), while five additional nonmanipulated subject variables were treated as independent variables, or clinician attributes which were measured (clinician multicultural awareness, multicultural knowledge, religiousness, spirituality, and view of faith helpfulness). Multicultural awareness was measured by the MCKAS-A subscale, multicultural knowledge by the MCKAS-K subscale, religiousness by the S-ROS- $\mathrm{I}_{\mathrm{R}}$ subscale, spirituality by the S-ROSIS subscale, and view of faith helpfulness by the RITSF-Help subscale. This study also included two dependent variables, prognosis and diagnosis. Prognosis was measured by 
the STPE-P2 subscale, and diagnosis was measured by the STPE-D subscale. All variables were measured as continuous variables except for faith type, which was measured as a categorical variable with two categories (religious and spiritual). Because magnitude estimation scaling was used to assess the magnitude of faith in the vignette versions and validated that they approximate interval measures, faith magnitude was also measured as a ratio variable, though represented by three categories (low, moderate, and high). A secular control vignette version (without a paragraph on religiousness or spirituality) was not included in the current study, as the low magnitude vignette versions were chosen because they were deemed to be more ecologically valid and authentic to the real world. Because the low religiousness version had approximately the same magnitude of faith as the low spirituality version, the two vignette versions were treated as nearly equivalent experimental alternatives to a control condition.

A total of 2 dependent variables, 3 independent variables, and 4 interaction effects were included in the design for each analysis. The dependent variables were diagnosis and prognosis. The 3 independent variables consisted of 2 treatment variables (faith magnitude and faith type), and one of 5 attribute variables (multicultural awareness, multicultural knowledge, religiousness, spirituality, and view of faith helpfulness). The 4 interaction effects consisted of one 2-way treatment-by-treatment interaction (magnitude*type), two 2-way attribute-by-treatment interactions ([attribute]*magnitude and [attribute]*type), and one 3-way attribute-by-treatment interaction ([attribute]* magnitude*type). The 2-way treatment-by-treatment interaction was included because it was essential to testing the Faith Combination hypothesis, while the 2-way and 3-way 
attribute-by-treatment interactions were included because they were necessary to test the Resilience hypothesis.

The Faith Type hypothesis tests the effect of the independent variable faith type on the dependent variables of diagnosis and prognosis. The Faith Magnitude hypothesis tests the effect of the independent variable faith magnitude on both dependent variables. The Faith Combination hypothesis tests the effects of the two independent variables faith magnitude and faith type, as well as their 2-way interaction, on the dependent variables. Finally, the Resilience hypothesis tests in 5 analyses the effects of 5 variable groupings on the dependent variables: (1) the 2 independent variables of faith magnitude and faith type, (2) the 2-way treatment-by-treatment interaction of the independent variables, (3) one of 5 clinician subject (attribute) variables (either multicultural awareness, multicultural knowledge, religiousness, spirituality, or view of faith helpfulness), (4) the two 2-way attribute-by-treatment interactions of faith magnitude and faith type with the attribute variable, and (5) the 3-way attribute-by-treatment interaction of the attribute variable with the 2-way interaction of faith magnitude and type. Five analyses test the Resilience hypothesis, one for each attribute variable.

The main advantage of a fractional factorial design is it allows interactions of interest among the attribute and treatment variables to be examined. It provides more information than designs using only one independent variable because it examines all the independent variables and all coded interactions simultaneously. If all the independent variables under investigation are related to the dependent variable(s), a factorial design also helps to reduce unexplained variance in these variables. The main disadvantage of this design is its complexity. Complex interactions between several independent variables 
can be difficult to interpret. Also, if some of the independent variables under investigation are unrelated to the dependent variable(s), then the unexplained variance in these variables may appear to increase, thus reducing the statistical power of the test (Heppner et al., 1999). 


\section{Chapter 4}

\section{Results}

To test for the presence of clinical judgment faith bias and clinician attributes which may moderate this bias, the data collected for the study were analyzed using regression analyses. First, variables were transformed and regression diagnostics were conducted to check for potential problems with missing data, multicollinearity, measurement error, specification error, nonlinearity, heteroscedasticity, and outliers. Next, five standard multivariate multiple regression analyses were conducted, to test the effects on the dependent variables (diagnosis and prognosis) of the treatment variables (faith magnitude and faith type), their interaction, one attribute variable (multicultural awareness, multicultural knowledge, religiousness, spirituality, or view of faith helpfulness), the 2-way interactions of the attribute variable with each treatment variable, and the 3-way interaction of the attribute variable with faith magnitude and type. Multivariate post hoc tests were planned for any regression that was statistically significant. The significance of each regression term was determined by inspection of the multivariate tests for the individual term, the F-tests of between-subject effects for each dependent variable, and the post hoc significance tests for each standardized Beta weight. 
Individual follow-up univariate F-tests of the dependent variables were planned (Jaccard \& Turrisi, 2003; Tabachnick \& Fidell, 2001). A post hoc statistical power analysis was also conducted, to ensure the current study had sufficient power to test the hypotheses of interest. The results of the statistical analyses are reported below.

\section{Regression Diagnostics}

Before conducting the primary analyses of interest, regression diagnostics were conducted to screen the data to determine whether they met the assumptions of these primary analyses. In regression analyses, several assumptions must be met in order for the mathematical calculations inherent in the analyses to yield meaningful results. The variables must often be transformed, the data must be complete, the variables must not be perfectly multicollinear, the data must have been measured correctly, the independent variables should contribute to reducing error variance in the prediction of the dependent variables, the data must be normally distributed, the variables must be linearly related, the variances of the error terms must be constant, and there must be no outliers in multivariate space (Berry \& Feldman, 1985). Each of these assumptions was examined during data screening (see Appendix M for a more detailed description).

A basic frequency analysis was conducted in SPSS to ensure the data were entered with all expected values for the scales. The categorical independent variable faith type was effects coded to make it appropriate for use in regression, the variable faith magnitude was coded as a continuous variable by using the magnitude estimation scaling values from Table 1 for each vignette version, and the continuous variables were standardized (or $z$-scored; Jaccard \& Turrisi, 2003). Missing data was not a problem (Berry \& Feldman, 1985), once the four surveys with substantial (i.e., > 20\%) missing 
responses were eliminated from the data set, resulting in a final sample size of $N=141$. Within this data set, only $3.92 \%$ of STPE responses, $1.57 \%$ of MCKAS responses, $1.61 \%$ of RITSF responses, and $2.54 \%$ of S-ROS responses were missing. Univariate collinearity was checked by inspecting the bivariate correlations among all the variables to ensure none are above 0.9 (Berry, 1993; Fox, 1991; see Table 3). No correlation exceeded 0.65 , with all but one correlation less than .50 . Multivariate multicollinearity was checked by regressing each variable on the remaining variables and checking to make sure no Tolerance statistic is less than 0.20, no VIF (Variance Inflation Factor) exceeds 4.0, and no Condition Index exceeds 30 (Belsley, Kuh, \& Welsch, 1980; Tabachnick \& Fidell, 2001; see Table 14 in Appendix M). No Tolerances were less than 0.48, no Condition Index exceeded 3, and no VIF exceeded 2.1. To control for measurement errors, a great deal of attention was given prior to the study to ensure reliable and valid instruments were selected to measure the variables of interest. Specification error was addressed by examining adjusted explained variance: "if this does not decrease when the variable in question is removed from the equation, it is clearly not playing any role in reducing the error variance and would therefore seem to be irrelevant" (Berry \& Feldman, 1985, p. 26). The adjusted $R^{2}$ values displayed in Table 15 in Appendix M suggest that although each variable was included for sound theoretical reasons, in the current data set, some variables do not contribute to the regressions being performed. Visual inspection of each variable's frequency histogram (see Figure 3 in Appendix M), as well as examination of each variable's skewness and kurtosis (see Table 16 in Appendix M), suggests each variable included in the current investigation is roughly normally distributed, indicating that non-normality did not appear to be a 
Table 3

Bivariate Correlations Among All Dependent and Independent Variables

\begin{tabular}{|c|c|c|c|c|c|c|c|c|}
\hline Variable & $N$ & $P$ & $D$ & $A$ & $K$ & $R$ & $S$ & $H$ \\
\hline Prognosis $(P)$ & 132 & 1.00 & & & & & & \\
\hline Diagnosis $(D)$ & 132 & .09 & 1.00 & & & & & \\
\hline Multicultural Awareness $(A)$ & 130 & .13 & .04 & 1.00 & & & & \\
\hline Multicultural Knowledge $(K)$ & 129 & $.21 *$ & .09 & $.23 * *$ & 1.00 & & & \\
\hline Religiousness $(R)$ & 134 & -.03 & .05 & -.05 & .16 & 1.00 & & \\
\hline Spirituality $(S)$ & 134 & .15 & .12 & -.01 & $.32 * *$ & $.64 * *$ & 1.00 & \\
\hline Faith Helpfulness $(H)$ & 129 & .13 & .12 & .08 & .14 & $.36 * *$ & $.43 * *$ & 1.00 \\
\hline
\end{tabular}

${ }^{*}$ Sig. $(1$-tailed $)<.05$

$* *$ Sig. $(1-$ tailed $)<.01$

problem for the current set of variables. Visual inspection of bivariate scatterplots of the relationships among all nonmanipulated variables did not reveal any nonlinear relationships between any of the current set of variables, suggesting that nonlinearity was not a problem. The assumption of homoscedasticity (as well as the assumptions of normality and linearity) was assessed by examining regression standardized predicted values plotted against regression standardized residuals for prognosis and diagnosis (see Figure 5 in Appendix M), which revealed no evidence of heteroscedasticity in the current data set (as well as no problems with non-normality or nonlinearity; Tabachnick \& Fidell, 2001). Finally, scatterplots depicting the bivariate relationships among variables were visually inspected for visible univariate outliers (see Figure 4 in Appendix M), while 
normal P-P plots of regression standardized residuals for the dependent variables were visually inspected for visible multivariate outliers (see Figure 6 in Appendix M), revealing no univariate or multivariate outliers. The highest Mahalanobis Distance for the dependent variables was 16.86, the highest Leverages for prognosis and diagnosis were 0.14 and 0.13 , respectively, and the highest Cook's D values for prognosis and diagnosis were 0.05 and 0.09 , respectively (see Table 17 in Appendix M). None of these values were very different from the other values for Mahalanobis Distances, Leverage, and Cook's D, suggesting no observations in the current data set are multivariate outliers.

\section{Data Analyses}

To analyze the effects of the treatment variables (faith magnitude and type), the attribute variables (multicultural awareness, multicultural knowledge, religiousness, spirituality, and view of faith helpfulness), and select interactions on the two continuous dependent variables of diagnosis and prognosis, the data from the current study were analyzed using five multivariate multiple regression analyses. Regression analysis is a statistical technique which permits the examination of the relationship between one continuous dependent variable and one independent variable (Pedhazur, 1997). Regression is typically used for two purposes: prediction and explanation. The primary goal of the current study is prediction - to determine how well the independent variables predict the dependent variables of clinician diagnostic and prognostic judgments, and which variables are important contributors to this regression equation. Multiple regression is an extension of regression analysis allowing for the exploration of the relationship between one continuous dependent variable and several continuous and/or categorical (e.g., dummy or effects coded) independent variables (Tabachnick \& Fidell, 
2001), while multivariate multiple regression is an extension of multiple regression exploring the relationship between multiple continuous dependent variables and multiple independent variables (Stevens, 2009). Multivariate multiple regression simultaneously tests multiple independent and dependent variables, controls for possible intercorrelations among these variables, and allows for follow-up tests to determine each independent variable's unique influence on each dependent variable (Haase \& Ellis, 1987; Lunneborg \& Abbot, 1983; Lutz \& Eckert, 1994; Stevens, 1986).

In the present study, the independent variables were the treatment variables of faith magnitude and faith type, and the attribute variables of multicultural awareness, multicultural knowledge, religiousness, spirituality, and view of faith helpfulness. The key interactions of interest were the 2-way treatment-by-treatment interaction of the independent variables (faith magnitude x faith type), ten 2-way attribute-by-treatment interactions of each independent variable with each attribute (multicultural awareness $\mathrm{x}$ faith magnitude, multicultural knowledge $\mathrm{x}$ faith magnitude, religiousness $\mathrm{x}$ faith magnitude, spirituality x faith magnitude, view of faith helpfulness $\mathrm{x}$ faith magnitude, multicultural awareness $\mathrm{x}$ faith type, multicultural knowledge $\mathrm{x}$ faith type, religiousness $\mathrm{x}$ faith type, spirituality $x$ faith type, and view of faith helpfulness x faith type), and five 3way attribute-by-treatment interactions of each attribute with the 2-way interaction of faith magnitude and type (multicultural awareness $\mathrm{x}$ faith magnitude $\mathrm{x}$ faith type, multicultural knowledge $\mathrm{x}$ faith magnitude $\mathrm{x}$ faith type, religiousness $\mathrm{x}$ faith magnitude $\mathrm{x}$ faith type, spirituality $x$ faith magnitude $x$ faith type, and view of faith helpfulness $x$ faith magnitude $\mathrm{x}$ faith type). The attribute-by-treatment interactions were of primary interest 
and allowed the Resilience hypothesis to be tested, while the treatment-by-treatment interaction allowed the Faith Combination hypothesis to be tested.

Five standard regression models were constructed with the variables and interactions described above and with 2 dependent variables: prognosis and diagnosis, measured by the STPE subscales. The Resilience hypothesis postulates that each clinician attribute independently moderates clinical judgment faith bias, without regard to the inclusion or exclusion of the other clinician attributes. The prior literature gives no indication as to how these attributes may operate in conjunction with one another in multivariate space, so each is examined separately. Rather than putting all the variables into a single model, then, five models were constructed, one for each clinician attribute. This regression strategy allowed for the direct examination in each model of the effects of one attribute variable, two treatment variables, one 2-way treatment-by-treatment interaction, two 2-way attribute-by-treatment interactions, and one 3-way attribute-bytreatment-by-treatment interaction on the two dependent variables. In this manner, five standard multivariate multiple regression analyses were performed, using the statistical program SPSS. The statistical significance of each regression was determined by inspection of Wilks' Lambda and the $p$ value of the associated $F$ distribution, which are provided in SPSS (Jaccard \& Turrisi, 2003).

The results of the five multivariate regressions are displayed in Tables 4, 5, 6, 7, and 8 . Regression 1 , for the attribute of multicultural awareness, was not statistically significant, Wilks Lambda $=.92, F(14,242)=0.71, p=.77$. This model predicted none of the variance in prognosis (Adjusted $R^{2}=-0.00$ ) or diagnosis (Adjusted $R^{2}=-0.03$ ). Regression 2, for the attribute of multicultural knowledge, was not statistically 
Table 4

Regression 1

Multivariate Tests of Significance $(S=2, M=2, N=591 / 2)$

\begin{tabular}{|c|c|c|c|c|c|c|}
\hline Effect & Wilks' & Approx. & Hypoth. & Error & $p$ & Eta \\
\hline & Lambda & $F$ & $\mathrm{DF}$ & DF & Value & Squared \\
\hline Regression 1 & .92 & .71 & 14 & 242 & .77 & .08 \\
\hline Intercept & 1.00 & .02 & 2 & 121 & .99 & .00 \\
\hline Magnitude (M) & .98 & 1.02 & 2 & 121 & .37 & .02 \\
\hline Type (T) & .98 & 1.08 & 2 & 121 & .34 & .02 \\
\hline $\mathrm{M} * \mathrm{~T}$ & 1.00 & .21 & 2 & 121 & .81 & .00 \\
\hline Awareness (A) & .98 & 1.19 & 2 & 121 & .31 & .02 \\
\hline $\mathrm{A} * \mathrm{M}$ & .99 & .59 & 2 & 121 & .56 & .01 \\
\hline $\mathrm{A} * \mathrm{~T}$ & 1.00 & .01 & 2 & 121 & .99 & .00 \\
\hline $\mathrm{A} * \mathrm{M} * \mathrm{~T}$ & .98 & .96 & 2 & 121 & .39 & .02 \\
\hline
\end{tabular}

Note. F statistic for Wilks' Lambda is exact.

Univariate F-tests with $(7,122) \mathrm{df}$

\begin{tabular}{lllllll}
\hline Variable & Sq. Mul. $R$ & Adj. $R^{2}$ & Hypoth. MS & Error MS & $F$ & $p$ Value \\
\hline Prognosis $^{\mathrm{a}}$ & .05 & .00 & .96 & 1.02 & .94 & .48 \\
Diagnosis $^{\mathrm{b}}$ & .02 & .00 & .44 & 1.07 & .41 & .89 \\
${ }^{\mathrm{a}} R^{2}=.05$ (Adjusted $\left.R^{2}=-.00\right)$ & & & & \\
${ }^{\mathrm{b}} R^{2}=.02$ (Adjusted $\left.R^{2}=-.03\right)$
\end{tabular}


Table 5

Regression 2

Multivariate Tests of Significance $(S=2, M=2, N=60)$

\begin{tabular}{|c|c|c|c|c|c|c|}
\hline Effect & Wilks' & Approx. & Hypoth. & Error & $p$ & Eta \\
\hline & Lambda & $F$ & DF & DF & Value & Squared \\
\hline Regression 2 & .88 & 1.15 & 14 & 244 & .32 & .12 \\
\hline Intercept & 1.00 & .02 & 2 & 122 & .98 & .00 \\
\hline Magnitude (M) & 1.00 & .73 & 2 & 122 & .48 & .00 \\
\hline Type (T) & .98 & 1.25 & 2 & 122 & .29 & .02 \\
\hline $\mathrm{M}^{* \mathrm{~T}}$ & .99 & .44 & 2 & 122 & .65 & .01 \\
\hline Knowledge (K) & .96 & 2.30 & 2 & 122 & .11 & .04 \\
\hline $\mathrm{K}^{*} \mathrm{M}$ & 1.00 & .32 & 2 & 122 & .73 & .00 \\
\hline $\mathrm{K}^{* \mathrm{~T}}$ & .97 & 1.60 & 2 & 122 & .21 & .03 \\
\hline $\mathrm{K}^{*} \mathrm{M} * \mathrm{~T}$ & .98 & 1.09 & 2 & 122 & .34 & .02 \\
\hline
\end{tabular}

Note. F statistic for Wilks' Lambda is exact.

Univariate F-tests with $(7,123) \mathrm{df}$

\begin{tabular}{lllllll}
\hline Variable & Sq. Mul. $R$ & Adj. $R^{2}$ & Hypoth. MS & Error MS & $F$ & $p$ Value \\
\hline Prognosis $^{\mathrm{a}}$ & .09 & .04 & 1.68 & .99 & 1.70 & .12 \\
Diagnosis $^{\mathrm{b}}$ & .04 & .00 & .70 & 1.03 & .68 & .69 \\
${ }^{\mathrm{a}} R^{2}=.09$ (Adjusted $\left.R^{2}=.04\right)$ & & & & & \\
${ }^{\mathrm{b}} R^{2}=.04$ (Adjusted $\left.R^{2}=-.02\right)$ & & & & &
\end{tabular}


Table 6

Regression 3

Multivariate Tests of Significance $(S=2, M=2, N=63)$

\begin{tabular}{|c|c|c|c|c|c|c|}
\hline Effect & Wilks' & Appro & Hypoth. & Error & $p$ & Eta \\
\hline & Lambda & $F$ & DF & DF & Value & Squared \\
\hline Regression 3 & .92 & .75 & 14 & 256 & .72 & .08 \\
\hline Intercept & 1.00 & .02 & 2 & 128 & .98 & .00 \\
\hline Magnitude (M) & .99 & .51 & 2 & 128 & .60 & .01 \\
\hline Type (T) & .99 & .61 & 2 & 128 & .55 & .01 \\
\hline $\mathrm{M} * \mathrm{~T}$ & .99 & .59 & 2 & 128 & .56 & .01 \\
\hline Religiousness (R) & .99 & .37 & 2 & 128 & .69 & .01 \\
\hline $\mathrm{R}^{*} \mathrm{M}$ & .96 & 2.83 & 2 & 128 & .06 & .04 \\
\hline $\mathrm{R} * \mathrm{~T}$ & .98 & 1.19 & 2 & 128 & .31 & .02 \\
\hline $\mathrm{R} * \mathrm{M} * \mathrm{~T}$ & 1.00 & .11 & 2 & 128 & .90 & .00 \\
\hline
\end{tabular}

Note. F statistic for Wilks' Lambda is exact.

Univariate F-tests with $(7,129) \mathrm{df}$

\begin{tabular}{lllllll}
\hline Variable & Sq. Mul. $R$ & Adj. $R^{2}$ & Hypoth. MS & Error MS & $F$ & $p$ Value \\
\hline Prognosis $^{\mathrm{a}}$ & .05 & .00 & .90 & 1.01 & .89 & .52 \\
Diagnosis $^{\mathrm{b}}$ & .04 & .00 & .69 & 1.03 & .67 & .70 \\
${ }^{\mathrm{a}} R^{2}=.05$ (Adjusted $\left.R^{2}=-.01\right)$ & & & & \\
${ }^{\mathrm{b}} R^{2}=.04$ (Adjusted $\left.R^{2}=-.02\right)$ & & & &
\end{tabular}


Table 7

Regression 4

Multivariate Tests of Significance $(S=2, M=2, N=63)$

\begin{tabular}{|c|c|c|c|c|c|c|}
\hline Effect & Wilks' & Approx. & Hypoth. & Error & $p$ & Eta \\
\hline & Lambda & $F$ & $\mathrm{DF}$ & $\mathrm{DF}$ & Value & Squared \\
\hline Regression 4 & .85 & 1.57 & 14 & 256 & .09 & .15 \\
\hline Intercept & 1.00 & .23 & 2 & 128 & .79 & .00 \\
\hline Magnitude (M) & .99 & .82 & 2 & 128 & .44 & .01 \\
\hline Type (T) & .99 & 1.00 & 2 & 128 & .37 & .01 \\
\hline $\mathrm{M} * \mathrm{~T}$ & .99 & .95 & 2 & 128 & .39 & .01 \\
\hline Spirituality (S) & .95 & 3.22 & 2 & 128 & .04 & .05 \\
\hline $\mathrm{S} * \mathrm{M}$ & .91 & 6.31 & 2 & 128 & .00 & .09 \\
\hline $\mathrm{S} * \mathrm{~T}$ & .99 & .99 & 2 & 128 & .38 & .01 \\
\hline $\mathrm{S} * \mathrm{M} * \mathrm{~T}$ & .99 & .45 & 2 & 128 & .64 & .01 \\
\hline
\end{tabular}

Note. F statistic for Wilks' Lambda is exact.

Univariate F-tests with $(7,129) \mathrm{df}$

\begin{tabular}{lllllll}
\hline Variable & Sq. Mul. $R$ & Adj. $R^{2}$ & Hypoth. MS & Error MS & $F$ & $p$ Value \\
\hline Prognosis $^{\mathrm{a}}$ & .13 & .09 & 2.58 & .92 & 2.80 & .01 \\
Diagnosis $^{\mathrm{b}}$ & .02 & .00 & .48 & 1.04 & .46 & .86 \\
${ }^{\mathrm{a}} R^{2}=.13$ (Adjusted $R^{2}=.09$ ) & & & & \\
${ }^{\mathrm{b}} R^{2}=.02$ (Adjusted $\left.R^{2}=-.03\right)$ & & & &
\end{tabular}


Table 8

Regression 5

Multivariate Tests of Significance $(S=2, M=2, N=601 / 2)$

\begin{tabular}{|c|c|c|c|c|c|c|}
\hline Effect & Wilks' & Approx. & Hypoth. & Error & $p$ & Eta \\
\hline & Lambda & $F$ & $\mathrm{DF}$ & $\mathrm{DF}$ & Value & Squared \\
\hline Regression 5 & .91 & .83 & 14 & 246 & .64 & .09 \\
\hline Intercept & 1.00 & .09 & 2 & 123 & .92 & .00 \\
\hline Magnitude (M) & .99 & .60 & 2 & 123 & .55 & .01 \\
\hline Type (T) & .98 & 1.54 & 2 & 123 & .22 & .02 \\
\hline $\mathrm{M} * \mathrm{~T}$ & .99 & .39 & 2 & 123 & .68 & .01 \\
\hline Helpfulness $(\mathrm{H})$ & .97 & 1.81 & 2 & 123 & .17 & .03 \\
\hline $\mathrm{H}^{*} \mathrm{M}$ & .98 & 1.16 & 2 & 123 & .32 & .02 \\
\hline $\mathrm{H}^{* \mathrm{~T}}$ & 1.00 & .18 & 2 & 123 & .84 & .00 \\
\hline $\mathrm{H}^{*} \mathrm{M} * \mathrm{~T}$ & .99 & .43 & 2 & 123 & .65 & .01 \\
\hline
\end{tabular}

Note. F statistic for Wilks' Lambda is exact.

Univariate F-tests with $(7,124) \mathrm{df}$

\begin{tabular}{lllllll}
\hline Variable & Sq. Mul. $R$ & Adj. $R^{2}$ & Hypoth. MS & Error MS & $F$ & $p$ Value \\
\hline Prognosis $^{\mathrm{a}}$ & .04 & .00 & .77 & 1.03 & .75 & .63 \\
Diagnosis $^{\mathrm{b}}$ & .05 & .00 & .90 & .93 & .96 & .46 \\
${ }^{\mathrm{a}} R^{2}=.04$ (Adjusted $\left.R^{2}=-.01\right)$ & & & & \\
${ }^{\mathrm{b}} R^{2}=.05$ (Adjusted $\left.R^{2}=-.00\right)$ & & & &
\end{tabular}


significant, Wilks Lambda $=.88, F(14,244)=1.15, p=.32$. This model predicted $4 \%$ of the variance in prognosis (Adjusted $R^{2}=0.04$ ) and none of the variance in diagnosis (Adjusted $R^{2}=-0.02$ ). Regression 3, for the attribute of religiousness, was not statistically significant, Wilks Lambda $=.92, F(14,256)=0.75, p=.72$. This model predicted none of the variance in prognosis (Adjusted $R^{2}=-0.01$ ) or diagnosis (Adjusted $R^{2}=-0.02$ ). Regression 4, for the attribute of spirituality, was not statistically significant, Wilks Lambda $=.85, F(14,256)=1.57, p=.09$. This model predicted $9 \%$ of the variance in prognosis (Adjusted $R^{2}=0.09$ ) and none of the variance in diagnosis (Adjusted $R^{2}=$ $-0.03)$. Regression 5, for the attribute of view of faith helpfulness, was also not statistically significant, Wilks Lambda $=.91, F(14,246)=0.83, p=.64$. This model predicted none of the variance in prognosis (Adjusted $R^{2}=-0.01$ ) or diagnosis (Adjusted $\left.R^{2}=-0.00\right)$. Because none of the regressions were statistically significant, no multivariate post-hoc tests were conducted.

After the primary analyses, a post hoc statistical power analysis was conducted, to explore if the regressions had sufficient power to test the hypotheses of interest. It was anticipated that power would be a problem, as the a priori power analysis predicted that if the effect size of each regression was no larger than a medium effect size of $r=.26$ $\left(f^{2}=.07\right)$, as the prior literature suggests, either more subjects would be required than participated in the current study (at least $N=197$ ), or the regressions would only obtain a power of .61 at a test-wise alpha of .01. The computer program G-Power (Faul, Erdfelder, Lang, \& Buchner, 2007) was used to calculate the achieved level of power of each regression, given 7 predictors, a test-wise alpha of .01, a sample size of 141, and obtained effect sizes of Eta Squared $=.08\left(f^{2}=.09\right)$, Eta Squared $=.12\left(f^{2}=.14\right)$, Eta 
Squared $=.08\left(f^{2}=.09\right)$, Eta Squared $=.15\left(f^{2}=.18\right)$, and Eta Squared $=.09\left(f^{2}=.10\right)$ for Regressions 1, 2, 3, 4, and 5, respectively. Regressions 1, 2, 3, 4, and 5 were found to have achieved power levels of $.47, .76, .47, .89$, and .54 , respectively. A conventional power level of at least .80 is usually recommended. This post hoc power analysis indicates that Regression 4 had sufficient power to detect a statistically significant effect if one exists, and Regression 2 had nearly sufficient power to detect one, but Regressions, 1,3 , and 5 had lower power to detect statistically significant effects, if they exist (Cohen, 1988).

The results of the five standard regressions indicated that none of these five models are predictive of clinical judgments. None of the hypotheses - neither the Faith Type hypothesis, nor the Faith Magnitude hypothesis, nor the Faith Combination hypothesis, nor the Resilience hypothesis - were supported by any of these five analyses, suggesting clinicians in the current sample did not make different diagnostic or prognostic judgments based on the magnitude, type, or social deviance of faith portrayed in the clinical vignettes they received. The diagnostic and prognostic judgments clinicians in the current sample made for religious cases were not significantly different than the judgments they made for spiritual cases, nor were they significantly altered by the magnitude of faith in the cases, or by the interaction of the type and magnitude of their faith. Furthermore, neither clinician multicultural awareness, nor clinician multicultural knowledge, nor clinician religiousness, nor clinician spirituality, nor clinician view of faith helpfulness appeared to influence the diagnostic or prognostic judgments clinicians in the current sample made for religious or spiritual cases at any magnitude of faith. These regressions indicated that the variables as currently modeled accounted for very 
small amounts of the variance in prognostic judgments and essentially none of the variance in diagnostic judgments. Alternatively, the low power of three of the analyses introduces the possibility that some of the variables did have a significant effect on other variables, but the effect was so small that the current study did not have the power to detect it.

\section{Summary}

The data analyses in the current study were not statistically significant. Five standard multivariate multiple regression analyses were performed to examine the effects of one attribute variable (either clinician multicultural awareness, multicultural knowledge, personal religiousness, personal spirituality, or view of faith helpfulness), two treatment variables (faith type and faith magnitude), one 2-way treatment-bytreatment interaction, two 2-way attribute-by-treatment interactions, and one 3-way attribute-by-treatment-by-treatment interaction on the dependent variables of prognosis and diagnosis. None of these regressions were statistically significant. The faith magnitude of cases did not appear to influence the diagnostic or prognostic judgments clinicians in the current sample made. Regressions 1, 3, and 5, however, had low power to detect statistically significant effects, if they exist. 


\section{Chapter 5}

\section{Discussion}

Clinical judgment is complex and multidimensional. Variability in clinical decision-making is attributable to a number of influences, as the judgments clinicians make when they are treating clients are multifaceted and influenced by multiple factors. This study focused on high magnitudes of client faith. Clinical judgment faith bias is the hypothesized tendency for clinicians to make more pathological judgments for clients with higher magnitudes of faith than for those with socially normative faith. The phenomenon of clinical judgment faith bias was not supported for the diagnostic or prognostic judgments made by a national sample of psychologists. The remainder of this chapter focuses on summarizing and discussing the current findings, explicating the limitations of this study, and discussing the implications of these findings for research and clinical practice.

This study has made a unique contribution to the literature by empirically investigating the social deviance of faith and its impact on clinical judgments. It is one of a small number of studies which experimentally investigated the relationship of clinical judgment and client faith and one of the only studies to connect a specific clinician bias 
to the social deviance of client faith. This study coins the term clinical judgment faith bias. Essentially, this study proposes a clinician bias, finds evidence that this bias is not taking place, and then through exploratory analyses finds evidence that the reverse of this bias might be taking place with certain clinicians. Like Feeser (1997), it is one of only a few studies to examine the interplay between religiousness and spirituality, to differentiate between them, and to control for the difference between them while investigating different magnitudes of client faith, instead of ignoring client spirituality or subsuming it under client religiousness. Insofar as most phenomena are not universal and occur only with certain clinicians, this study also had the benefit of investigating the contribution of multiple interacting clinician variables to clinical judgment faith bias: not only clinician religiousness and spirituality (to compliment the client religiousness and spirituality being manipulated) but also clinician view of faith helpfulness, multicultural awareness, and multicultural knowledge with two types of client faith.

\section{Findings}

Surveying by mail 141 psychologists in clinical practice, four hypotheses were empirically tested using multiple regression analyses. The first hypothesis, the Faith Type hypothesis, was not supported. The diagnostic and prognostic judgments clinicians in the current sample made for religious cases were not significantly different than the judgments they made for spiritual cases. This suggests clinical judgment faith bias is not the result of clinician bias towards the religious or spiritual qualities clients presented. It was predicted that the presence of religious beliefs alone might be enough to activate salience bias and the representativeness heuristic for some clinicians, leading them to 
invoke stereotypes of religious people and make biased judgments (Kahneman \& Tversky, 1973; Nisbett \& Ross, 1980; Taylor, 2006). This was not the case.

The second hypothesis, the Faith Magnitude hypothesis, was also not supported. Clinicians in the current sample did not make different diagnostic or prognostic judgments as the magnitude of the case increased. Behavior decision-making theory predicts that clinicians using the representativeness heuristic as a cognitive shortcut might automatically access stereotypes of religious people and make unconscious judgments based on these stereotypes, without considering the base rates of no higher rates of psychopathology in this population, and that salience bias might bring these stereotypes to mind as the magnitude of faith increases, making clinicians increasingly likely to show salience bias and use the representativeness heuristic as a cognitive shortcut (Kahneman \& Tversky, 1973; Nisbett \& Ross, 1980; Taylor, 2006). The lack of support for the Faith Magnitude hypothesis suggests clinical judgment faith bias is not the result of clinician bias towards the magnitude or social deviance of the cases presented.

The synergistic interaction of the first two hypotheses in the third hypothesis, the Faith Combination hypothesis, was furthermore not supported. Client type and magnitude of faith did not interact to influence the judgments of clinicians. This indicates that no faith bias was found in the clinical judgments of the psychologists surveyed here. Contrary to what behavior decision-making theory suggests, the stereotype of the mentally ill religious fanatic apparently did not come to mind when the clinicians in the current study were evaluating highly religious or spiritual clients. Neither client religiousness alone nor the combination of religiousness and high magnitudes of faith was sufficient to make these stereotypes salient to the clinicians in the current study - or 
if these stereotypes did come to mind, salience bias and the representativeness heuristic did not lead clinicians to decide these stereotypes are representative of highly religious or spiritual people or ignore the relevant base rates of pathology among highly religious people, and these clinicians instead discounted this stereotype.

Finally, the fourth hypothesis, the Resilience hypothesis, was not supported for any clinician attributes measured here. It was anticipated based on prior research that certain clinician characteristics might confer resilience to clinical judgment faith bias, moderating the impact of client faith magnitude, client faith type, or their interaction on clinical judgments. Neither clinician multicultural awareness, clinician multicultural knowledge, clinician personal religiousness, clinician personal spirituality, nor clinician view of faith helpfulness appeared to influence either the diagnostic or prognostic judgments clinicians in the current sample made, regardless of the type of faith portrayed in the cases, or the magnitude of faith present in the cases.

The results of the primary analyses are consistent with the mixed and often conflicting findings in the prior literature. While some studies (e.g., Hillowe, 1985; Houts \& Graham, 1986; O'Connor \& Vandenberg, 2005) found evidence of clinical judgment faith bias, other studies (e.g., Gartner, J., Harmatz, M., Hohmann, A., Larson, D., \& Gartner, 1990a, 1990b; Jones, 1991; Sanderson, Vandenberg, \& Paese, 1999) found no such evidence of bias or conflicting findings even within the same study. Again, the conflicting results which have been found are often due to different ways in which religiousness has been measured, or different aspects of religion which are examined. The results found in the current study are due to the instruments and variables used here, and the lack of statistical significance may be idiosyncratic to these variables and instruments. 
The results found here are also consistent with the curvilinear relationship between religiousness and pathology (c.f. Eliassen, Taylor, \& Lloyd, 2005; IngersollDayton, Krause, \& Morgan, 2002; Koenig, Ford, George, Blazer, \& Meador, 1993; Perkins, 1992; Pressman, Lyons, Larson, \& Gartner, 1992; Ross, 1990; Schnittker, 2001; Wink \& Scott, 2005). The dependent measures used in the current study, however, were not sensitive enough to pick up a curvilinear relationship.

The results of the current study are consistent with the literature finding no relationship between religiousness and clinical judgments of mental health (Davis, Kerr, \& Kurpius, 2003; Harris, Schoneman, \& Carrera, 2002; Koenig, Ford, George, Blazer, \& Meador, 1993; Koenig, George, Blazer, Pritchett, \& Meador, 1993; Miller \& Kelley, 2005; Oman \& Thoresen, 2005; Pfeifer \& Waelty, 1999), no relationship between religiousness and clinical judgments of anxiety (Koenig, 1998, 2005), and no relationship between religiousness and clinical judgments of OCD (Greenberg \& Shefler, 2002; Hermesh, Masser-Kavitsky, \& Gross-Isseroff, 2003; Tek \& Ulug, 2001; Okasha, Lotaief, Ashour, El Mahalawy, Seif El Dawla, \& El-Kholy, 2000). Sanderson, Vandenberg, and Paese (1999) found when 67 mental health professionals were asked to rate 18 case vignettes on their degree of mental health, clinicians did not make judgments based on the religious content of the vignettes. Studies by Gartner and colleagues (1990a, 1990b) found 363 clinical psychologists did not give different severity ratings on the Clinical Judgment Scale to members of different religious groups. Similarly, Jones (1991) found nonreligious counselors rated the religious cases as having the worst prognoses, but other counselors did not give significantly different prognostic ratings to cases depicting clients who were nonreligious, very religious, or religiously doubtful. 
Studies by Gartner and colleagues (1990a, 1990b) and Yossifova and Loewenthal (1999) found when clinicians were asked to give diagnoses for nearly equivalent cases, clients with high religiousness and extreme ideologies were more likely to be judged as having obsessional symptoms than clients with low religiousness or less extreme ideologies, but they were less likely to be diagnosed with Generalized Anxiety Disorder. Since the correct diagnosis in the current study was Generalized Anxiety Disorder (and most clinicians picked up on this), the magnitude of faith depicted in the highly religious and spiritual vignette versions may not have been extreme enough to yield similar results in this study.

Perhaps the religiousness depicted in the vignette versions was not salient enough to most of the participants in the current sample to lead to salience bias or to the invocation of the representativeness heuristic. Religious extremism is the most salient kind of socially deviant religiousness, such as Terry Jones' threats to hold a Qu'ran burning, the Westboro Baptist Church's anti-gay protests at military funerals, the Branch Davidian religious cult, the Heaven's Gate religious group, Jim Jones' People's Temple cult, and the alleged mind control techniques of the International Society for Krishna Consciousness. The highly religious and spiritual vignette versions were much more subdued in content than these sensationalized examples of religious extremism. These vignette versions were developed specifically to portray realistic depictions of faith which highly religious and spiritual people agreed represented believable, real-worldauthentic depictions of their faith. These highly religious and spiritual vignette versions may have been simply not extreme enough, or not close enough to the religious extremism depicted in the media - the Terry Joneses, Westboro Baptist Churches, Branch 
Davidians, Heaven's Gates, Jim Joneses, and Andrea Yateses of the world - the kinds of religious extremism which are most salient in the minds of most Americans. Thus, salience bias may not have come into play in the current sample - and if salience bias was not activated, then perhaps the representativeness heuristic was not employed, either.

There were very low correlations among all the variables measured in the current study. Though previous research indicated potential connections between clinical judgments and the manipulated variables, the measured attributes, and their interaction, only very weak connections were found here. Consequently, because the intercorrelations among variables were so low, any tendency towards bias which may have existed was obscured. The power of three of the regressions was also low - too low to detect a significant effect if one exists but is small. Two of the regressions - Regressions 2 and 4 - had sufficient power to detect the medium effect size that was anticipated, so these analyses reasonably show that there is likely no significant effect to be detected with the clinician attributes of multicultural knowledge and spirituality. The low power of the other three analyses, however, introduces the possibility that some of the other variables did have a significant effect on each other, but the effect was so small that these regressions did not have the power to detect it.

The findings of statistical nonsignificance for the Faith Type, Faith Magnitude, and Faith Combination hypotheses are somewhat surprising, given the previous clinical judgment literature reviewed in Chapters 1 and 2 which suggests that each of the variables may be associated with bias. These findings suggest one of three possibilities. One is that the model is improperly specified - that clinical judgment faith bias does exist in some form but was simply not detected due to shortcomings in the model or 
measurement of the variables or the choice of the dependent variables or something like that. Another possibility is that clinical judgment faith bias does exist, but only with the regressions involving the clinician attributes of multicultural awareness, religiousness, and view of faith helpfulness - and it was precisely those regressions which did not have the power to detect significant effects. A third possibility - and the more likely possibility given the attention to detail that was put into this study and the nonsignificant findings in the two regressions that did have sufficient power - is that clinical judgment faith bias was not shown in the current sample. This finding, while surprising, is reassuring because it speaks well to the profession of psychology, suggesting that clinicians do not make judgments biased by client faith. Perhaps multicultural training is working well. Perhaps clinicians are being mindful of their own personal biases. Perhaps clinicians are simply attending to the most relevant details when making a diagnosis or estimation of prognosis and rightly assuming that faith is not related to therapeutic outcomes when it is not a symptom of dysfunction. For whatever reason, the psychologists in the current sample do not appear to have displayed clinical judgment faith bias.

\section{Limitations}

The current research project has a number of limitations: the low power of the statistical analyses, the multiple analyses run, the lack of a control group, the small sample size, the validity of the STPE, the transparency of the survey and selection bias, the heavy demand characteristics, the poor return rate, the clinical analogue procedure, the sample (non)representativeness, the controversial subject matter, self-report bias, the interaction of setting and treatment, the measurement of clinician religiousness and spirituality, the vignette's demographics, and the use of Dillman's (2007) Tailored 
Design method. These caveats should be considered when interpreting the results presented here, as they potentially limit the generalizability of the conclusions presented above to psychologists, clients, and clinical judgments as a whole.

As noted previously, a primary limitation of the current study was the low power of the statistical analyses. Because five analyses were conducted, alpha was divided by five, using a Bonferroni correction, which greatly reduced the power of each of the five analyses. Consequently, three of the regressions may not have had sufficient power capable of detecting the effects in those analyses. Had the power level of the current study been higher, perhaps some effects would have been detected in those three regressions. The low power of those three statistical analyses was a significant limitation of this study (Tabachnick \& Fidell, 2001).

Another significant limitation of the current study - and one contributing to the low power of the analyses - was the multiple analyses run. Because of the number of variables of interest and the hypotheses which were tested, multivariate multiple regression was employed to minimize the number of analyses conducted, but multiple analyses were nevertheless necessary to test alternative models of the data. Running multiple analyses on the same data set could have led to idiosyncratic results or spurious findings - and lowered the power of all the analyses to detect significant effects.

The lack of a control group in the current study posed another notable limitation of this study. Two low magnitude vignette versions with approximately the same low magnitude of faith were chosen as alternatives to a control version, as they were deemed to be more ecologically valid and authentic to the real world. Because a true control group was not included in this study, however, it is possible that clinicians responded in 
exactly the same way to the religious versions as they did to the spiritual versions, while they might have responded differently to a secular version than to either the religious or spiritual versions. Because a control condition was not included, this alternative explanation cannot be ruled out.

The sample size was small for a study which included so many variables and effects. The initial power analysis estimated at least 197 participants would be necessary to detect a medium anticipated effect size for the multivariate effects using the planned multiple regression analyses, and the follow-up power analysis indicated that three of the regressions had low power. The current sample size of 141 was just barely large enough to give two of the statistical analyses conducted sufficient power to detect significant differences if they exist, and not large enough to give the remaining three analyses sufficient power. The difficulty of collecting data from additional participants made doing so prohibitive in the current study. Given the complexity of the research design, the number of variables employed, and the power of the analyses employed, though, a larger sample size would have been preferable and would have given additional power to the analyses conducted.

The validity of the Schedule for Therapist's Prognostic Expectations (STPE) was defensible but questionable. In spite of efforts to minimize the impact of a printing error in the questionnaire and statistical checks against prior research supporting the validity of the current administration of the scale, the anchors for several STPE items were nevertheless improperly printed, calling into question the validity and interpretability of this scale. The items which were most affected were not used in the current study. Still, the administration of the scale deviated from prior research, "testing the limits" of this 
scale. Several participants commented on this instrument's anchors, finding them confusing. This was a notable limitation.

The transparency of the survey and selection bias may have influenced the results of this study (Heppner et al., 1999). Although the questions were ordered to minimize selection bias by masking the content of the questionnaire, it was still readily apparent early in the questionnaire that respondents were being asked many questions about religion and spirituality. Combined with the controversial subject matter and demand characteristics of the study, those clinicians who participated may have had a particular interest in the topic of faith - or those who did not participate may have had a particular dislike for the topic. The transparency of the survey may have led to some selection bias as participants selected whether or not to complete the survey, and consequently the sample may not have been a random or representative sample of American psychologists.

Another limitation of the current study was the demand characteristics of the mail survey (Heppner et al., 1999). The questionnaire which was sent to participants contained 5 separate surveys with 14 distinct scales, was 142 questions long, and required respondents to read a two-paragraph vignette. This placed great demand on respondents, which some participants may have found intimidating and most likely found time consuming. In the qualitative comments made by participants, more than one person commented on the length of the survey. It is likely many of those who were surveyed decided not to participate because of length.

The return rate for the current study was somewhat poor, indicating attrition may have been a problem. The return rate of $35 \%$ was adequate for an experimental study and typical for a clinical judgment study (c.f. Spengler \& Strohmer, 1994; Walker, 1999). 
Such a low return rate, however, is too low to assume the current sample is representative of all psychologists nationwide. The poor return rate may have been due to the survey's demand characteristics.

Another potential limitation was the use of a clinical analogue procedure. The vignettes which were used did not depict a real client, but instead were generated to allow for the experimental manipulation of client faith. This allowed for greater experimental control, and expert raters validated that these vignettes represented accurate, true-to-life depictions of authentic religious and spiritual faith. Nevertheless, the use of a clinical analogue procedure rather than studying real-life examples of clinical decision-making with actual clients resulted in a potential decrease in external validity and a potential loss of generalizability to the real world (Heppner, Kivlighan, \& Wampold, 1999).

The current sample may not have been representative of psychologists as a whole. The current sample had a larger age range, included proportionally more female psychologists and psychologists with Psy.D.'s, and included proportionally less African American psychologists, psychologists of other ethnic backgrounds, psychologists with Ph.D.'s, and clinical psychologists than a representational sample of psychologists was anticipated to contain based on national data from the APA (APA Directory Survey, 1999). Therefore, the current sample may not adequately represent all psychologists in the United States. The somewhat low return rate may be partly to blame for this finding.

An additional factor which may have discouraged some clinicians from participating was the controversial subject matter of the survey. The questionnaire addressed religion, spirituality, personal beliefs, race, and multicultural competence, asking some fairly personal questions. Also, some participants may have found part of 
the questionnaire to be offensive - particularly items whose endorsement would signify low multicultural competence (e.g., "I feel all the recent attention directed toward multicultural issues in counseling is overdone and not really warranted") or low view of faith helpfulness (e.g., "Religious belief tends to detract from one's intellectual development" or "Religious belief tends to be a way of avoiding reality"). Although the questions were ordered to minimize the impact of the more controversial subjects (and so participants would be less likely to guess the survey's intention based on the content of the questions until after they had made their clinical judgments), some of the items could be read as potentially offensive. Some respondents commented they found some items offensive, while some commented that completing the questionnaire brought up strong feelings for them, both for and against religion. Still others commented they objected to the reduction of faith or cultural competence to 5-point Likert-type items.

Self report (or mono-method) bias was another limitation of this study. Because of the mail survey method used, all instruments were self-report measures, and most of the questions were Likert-type. This may have introduced a systematic bias into the results, simply because of the method used (Heppner et al., 1999).

As in all survey research, the interaction of setting and treatment poses a minor limitation to the current study. There was no consistency in test setting for the current research due to the survey method; respondents could complete the questionnaire at home, at work, in their car, while waiting at the airport, or anywhere else they chose. This may have increased the variability in participants' responses, making it harder to find significant results (Heppner et al., 1999; Tabachnick \& Fidell, 2001). 
Another potential limitation was the measurement of clinician religiousness and spirituality. A perennial problem in any study attempting to measure these variables, the operationalization and measurement of religiousness and spirituality is particularly problematic, since these concepts are, by some definitions, ineffable or indefinable (Powers, 2003). Literally hundreds of scales have been developed to measure various aspects of religiousness and spirituality (Hill \& Hood, 1999). Here, the Spiritual-Intrinsic and Religious-Intrinsic subscales of the Spiritual-Religious Orientation Scale were selected to measure clinician religiousness and spirituality broadly, based on conceptual reasons outlined earlier. These subscales are moderately to highly correlated with other measures of religiousness and spirituality (Hill \& Hood, 1999; Peterson, 2001). Had other scales been chosen, the results may have been slightly to significantly different.

The vignette's demographics pose another limitation to the generalizability of the current study. The vignette depicted a White/Caucasian female client with Baptist religious faith or a nonreligious unspecified spirituality. Therefore, the results may not be generalizable to non-White clients, male clients, or clients from other religious faiths.

Finally, a practical limitation of the current study was its use of Dillman's (2007) Tailored Design method for mail and internet surveys. Using this method was both expensive and labor intensive, as this method employs real stamps, dollar bill incentives, and five contacts. Surveying each participant cost $\$ 0.42$ in stamps for the prenotice letter, $\$ 1.01$ in stamps and $\$ 1.00$ incentive for the first survey mailing, $\$ 0.42$ in stamps for the thank you letter, $\$ 1.01$ in stamps for the second survey mailing, and another $\$ 0.42$ in stamps for the final notice letter, plus the cost of stationary (4 pages of resume paper), envelopes ( 4 business envelopes and 2 booklet envelopes), and printing the 8-page survey 
booklets ( 2 per participant). 400 prenotice letters, 400 first survey mailings, 400 thank you letters, over 300 second survey mailings, and approximately 250 final notice letters were sent, at a total cost of over $\$ 1200$ in postage, over $\$ 500$ in stationary and printing costs, and $\$ 400$ in incentives. Each of the approximately 1800 mailings and 700 return envelopes were addressed, stamped, assembled, and sealed individually, by hand (with the aid of printed address labels). According to Dillman (2007), using this methodology is likely to yield a higher return rate than using other, less expensive and less labor intensive methods. Nevertheless, a sufficiently high return rate might have been achieved by using less expensive methods (e.g., an internet survey), perhaps by surveying a larger number of participants (but only contacting them once or twice). Furthermore, the use of dollar bills as thank-you incentives may have been unnecessary. More than 30 participants returned their dollar to the researcher in the return envelope provided - most without completing the survey. It is difficult to determine what this action meant apart from declining to participate. Perhaps they were offended. Perhaps they simply did not wish to participate and hoped to negate the social influence of the gift by giving it back to the researcher, after which they felt no obligation to participate. Perhaps they felt bad for the researcher, a graduate student whom they assumed (correctly) did not have much money. As these nonrespondents did not indicate why they were returning their dollars, it is impossible to tell. The respondents who participated, however, made a few comments about the dollars in their qualitative comments. Some responded positively to the receipt of this incentive (one wrote, "Thanks for the dollar (nice idea)"), while one commented, “I resent being sent $\$ 1.00$," and another said, "I am returning your dollar bill. Thanks, but 
as a student, I'm sure you need it more than I do!" Thus it is unclear what benefit, if any, the inclusion of the dollars had on the study.

\section{Research Implications}

With all of the caveats noted in the previous section, the findings of the current study may nonetheless have some tentative implications for future research. The present study examined multiple interacting factors to see which combination contributes to the judgments clinicians make, and it failed to confirm the existence of clinical judgment faith bias. Whereas much previous research has focused on the specific content of client faith and yielded conflicting results regarding faith's contribution to clinical bias, this study found evidence that the proposed mechanism underlying this bias - not faith itself, but the moderating variable of social deviance - was not acting to influence clinical judgments in the current sample.

The clinical analogue method used in the current study did not capture the proposed clinical judgment faith bias. A more sensitive method might have more success in capturing this proposed bias, such as in-vivo surveying or qualitative content analysis of actual clinical decisions.

The current study proposed and eliminated five regression models for future research. It eliminated two dependent variables which were not influenced by any of the attribute or treatment variables measured or manipulated. It also eliminated several independent and attribute variables as well as several interaction variables which had no influence on clinical judgment. In all five regression models, client faith magnitude, client faith type, clinician multicultural awareness, clinician multicultural knowledge, clinician religiousness, clinician spirituality, clinician view of faith helpfulness, and 
select interactions altogether accounted for only a very small percentage of the variance in clinical judgments. Future research should focus on other clinician variables which influence clinical decision making (such as gender, experience, treatment modality, theoretical orientation, and the like) and examine how they may interact with client faith magnitude, clinician spirituality, and clinician multicultural knowledge to influence clinical judgments. Future research can also focus on other measures of clinical judgment, including severity and appropriateness, as the choice of dependent measures invariably serves to limit the results.

The current study highlights the importance of differentiating between religiousness and spirituality in research. Consistent with theory and research which casts these variables as highly correlated but distinct constructs with differential influences on clinical outcomes, clinicians rated themselves as significantly more spiritual than religious. On the other hand, consistent with theory and research which casts these variables as different aspects of the same underlying construct, the present study found no difference in how client religiousness and spirituality influenced prognostic or diagnostic judgments. Future research should investigate spirituality and religiousness as distinct clinician characteristics while examining them as a unitary client characteristic.

This research project also found adequate psychometric support for three previously under-researched scales: the Spiritual - Religious Orientation Scale (S-ROS), the Religious Issues in Therapy Survey Form (RITSF), and the Schedule for Therapist Prognostic Expectations (STPE). This lends credibility for their use in future studies. It might be of interest for future research to investigate the influence of other client and clinician variables on clinical judgment faith bias. In particular, the race and 
gender of clients and clinicians alike might interact with client faith to influence clinicians' judgments. In general, women tend to be more religious than men (Stanard, Sandhu, \& Painter, 2000), while religion is often an integral part of African American and Hispanic American cultures in a way which is often not characteristic of other American cultures (Constantine, Lewis, Conner, \& Sanchez, 2000). It stands to reason, therefore, either or both of these variables might have an influence on clinical judgment. Future clinical judgment research should explore these possibilities.

\section{Clinical Implications}

The current findings have some tentative implications for clinical practice as well. Most importantly, they suggest that most clinicians' diagnostic and prognostic judgments may not be susceptible to clinical judgment faith bias. Contrary to the findings in previous research suggesting a possible faith bias, the anticipated bias was not found here. These results are encouraging, as they suggest that either this proposed clinical judgment faith bias does not exist, or it exists but did not adversely impact the clinical judgments a national sample of practicing psychologists made of the vignettes employed here. Perhaps faith bias used to exist but no longer biases the judgments of well-trained clinicians today, or perhaps the multicultural competence of modern clinicians imparted resistance to faith bias and did so throughout the entire sample so that no effect was found. Clinicians must continue to be mindful when treating clients with high magnitudes of faith, so they do not inadvertently pathologize highly religious and spiritual clients. The current results, however, are encouraging, as they suggest most clinicians are being mindful of client faith and are not likely to misdiagnose them or unduly pathologize their 
prognoses. Ostensibly, this reflects well on the clinical training of the psychologists in this national sample, that they are not making the judgment errors proposed in this study.

The current study found that while clinician religiousness and spirituality have the potential to influence the judgments clinicians make for clients, they do not appear to be doing so in the current sample. It speaks well of the current sample, then, that these mental health professionals are apparently being mindful of how their religious and spiritual beliefs and values influence their general outlook.

\section{Conclusion}

In conclusion, clinical judgment is a complex phenomenon influenced by multiple factors. The diagnostic and prognostic judgments clinicians make do not appear to be influenced by the type or magnitude of clients' faith, and these judgments do not appear to be influenced by clinician multicultural awareness, multicultural knowledge, religiousness, spirituality, view of faith helpfulness, or select attribute-by-treatment interactions. Clinicians do not appear to respond differently to spiritual clients than to religious clients when making diagnostic or prognostic judgments, and they do not appear to respond differently to highly religious or spiritual clients than to clients with moderate or low religiousness or spirituality. Clinical judgment faith bias was not found in the current sample, and no clinician attributes were found which imparted resilience to it. In light of prior research suggesting a possible faith bias, the current results are encouraging, as they suggest that this proposed bias did not adversely impact the clinical judgments a national sample of practicing psychologists made of the vignettes employed here. There are many other client and clinician variables which influence the judgments clinicians make apart from clinician multicultural knowledge and the interaction of clinician 
spirituality and the magnitude of client faith. These variables account for only a small proportion of variance in outcome in clinical decision making - if they account for any at all. Nevertheless, these are phenomena which appear to have some subtle influence on clinical judgment, with implications for research and clinical practice. As such, these phenomena should not be ignored, for the sake of improving outcomes of prognostic judgments and aiding in more accurate clinical decision making. 


\section{References}

Abramowitz, J. S., Deacon, B. J., Woods, C. M., \& Tolin, D. F. (2004).

Association between protestant religiousness and obsessive-compulsive symptoms and cognitions. Depression and Anxiety, 20, 70-76.

Aiken, L., \& West, S. (1991). Multiple regression. Newbury Park, CA: Sage.

Akers, R. (2000). Criminological theories. Los Angeles: Roxbury.

Allport, G. W., \& Ross, J. M. (1967). Personal religious orientation and prejudice. Journal of Personality and Social Psychology, 5, 423-443.

Alonso, P., Menchon, J. M., Pifarre, J., Mataix-Cols, D., Torres, L., Salgado, P., \& Vallejo, J. (2001). Long-term follow-up and predictors of clinical outcome in obsessive-compulsive patients treated with serotonin reuptake inhibitors and behavioral therapy. Journal of Clinical Psychiatry, 62, 535-540.

Altemeyer, B., \& Hunsberger, B. (2005). Fundamentalism and authoritarianism. In R. F. Paloutizian \& C. L. Park (Eds.), Handbook of the psychology of religion and spirituality (pp. 378-393). New York: Guilford Press.

American Psychiatric Association. (1980). Diagnostic and statistical manual of mental disorders ( $3^{\text {rd }}$ ed.). Washington, DC: Author. 
American Psychiatric Association. (2000). Diagnostic and statistical manual of mental disorders ( $4^{\text {th }}$ ed., text revision). Washington, DC: Author.

American Psychological Association. (1999). APA Directory Survey. Washington, D.C.: Author.

American Psychological Association. (2002). Ethical principles of psychologists and code of conduct. Washington, DC: Author.

Amira, S., Abramowitz, S. I., \& Gomes-Schwartz, B. (1977). Socially-charged pupil and psychologist effects on psychoeducational decisions. Journal of Special Education, 11, 433-440.

Anthony, D., \& Robbins, T. (1997). Religious totalism, exemplary dualism and the Waco tragedy. In T. Robbins \& S. J. Palmer (Eds.), Millennium, messiahs, and mayhem (pp. 261-284). New York: Routledge.

Argyle, M. (1999). Causes and correlates of happiness. In D. Kahneman, E. Diener, \& N. Schwarz (Eds.), Well-being: The foundations of hedonic psychology (pp. 353-373). New York: Russell Sage Foundation.

Argyle, M., \& Beit-Hallahmi, B. (1975). The social psychology of religion. London: Routledge.

Armstrong, T. D. (1995, August). Exploring spirituality: The development of the Armstrong Measure of Spirituality. Paper presented at the annual convention of the American Psychological Association, New York, NY.

Arredondo, P. (1999). Multicultural counseling competencies as tools to address oppression and racism. Journal of Counseling and Development, 77, 102-108. 
Baker, M. \& Gorsuch, R. (1982). Trait anxiety and intrinsic-extrinsic religiousness. Journal for the Scientific Study of Religion, 21, 119-122.

Bambgose, O., Edwards, D., \& Johnson, S. (1980). The effects of race and social class on clinical judgment. Journal of Clinical Psychology, 36, 605-609.

Bar-Hillel, M. (1980). The base-rate fallacy in probability judgments. Acta Psychologica, 44, 211-233.

Barlow, D. H., \& Durand, V. M. (1995). Abnormal psychology: An integrative approach. Pacific Grove, CA: Brooks/Cole.

Baron, R. A., \& Byrne, D. (1997). Social psychology ( $8^{\text {th }}$ ed.). Boston, MA: Allyn and Bacon.

Batson, C. D., \& Ventis, W. L. (1982). The religious experience: A socialpsychological perspective. New York: Oxford University Press.

Batson, C. D., Schoenrade, P., \& Ventis, W. L. (1993). Religion and the individual: A social psychological perspective. New York: Oxford University Press.

Bear, D. M. (1982). Interictal behavior in hospitalized temporal lobe epileptics: Relationship to idiopathic psychiatric syndromes. Journal of Neurology, Neurosurgery, and Psychiatry, 45, 481-488.

Belsley, D. A., Kuh, E., \& Welsch, R. E. (1980). Regression diagnostics: Identification of influential data and sources of collinearity. New York: John Wiley \& Sons.

Beck, C. (1986). Education for spirituality. Interchange, 17, 148-156.

Becker, L. A. (2000, March). Effect size calculators. Retrieved May 23, 2008 from http://web.uccs.edu/lbecker/Psy590/escalc3.htm. 
Belaire, C., \& Young, J. S. (2002). Conservative Christians' expectations of nonChristian counselors. Counseling and Values, 46, 175-187.

Bellah, R. N. (1970). Beyond belief. New York: Harper \& Row.

Benefee, L. M., Abramowitz, S. I., Weitz, L. J., \& Armstrong, S. H. (1976).

Effects of patient racial attribution on Black clinicians' inferences. American Journal of Community Psychology, 4, 263-273.

Benjamin, P., \& Looby, J. (1998). Defining the nature of spirituality in the context of Maslow's and Rogers's theories. Counseling \& Values, 42, 92-100.

Berger, H. A. (1999). A community of witches: Contemporary Neo-Paganism and witchcraft in the United States. Columbia, SC: University of South Carolina Press.

Berger, H. A., Leach, E. A., \& Shaffer, L. S. (2003). Voices from the Pagan census: A national survey of witches and Neo-Pagans in the United States. Columbia, SC: University of South Carolina Press.

Bergin, A. E. (1980). Psychotherapy and religious values. Journal of Consulting and Clinical Psychology, 48, 642-645.

Bergin, A. E. (1991). Values and religious issues in psychotherapy and mental health. American Psychologist, 46, 394-403.

Bergin, A. E., \& Jensen, J. P. (1990). Religiosity of psychotherapists: A national survey. Psychotherapy: Theory, Research, Practice, Training, 27, 3-7.

Berman, R., Lauritson, P., Williams, B., \& Carson, D. (1994). Star Trek: Generations [motion picture]. United States: Paramount Pictures Corporation.

Berry, W. D. (1993). Understanding regression assumptions. Thousand Oaks, CA: Sage. 
Berry, W. D., \& Feldman, S. (1985). Multiple regression in practice. Thousand Oaks, CA: Sage.

Beutler, L. E., \& Bergan, J. (1991). Value change in counseling and psychotherapy: A search for scientific credibility. Journal of Counseling Psychology, 38, $16-24$.

Blaine, B., \& Crocker, J. (1995). Religiousness, race, and psychological wellbeing: Exploring social psychological mediators. Personality and Social Psychology Bulletin, 21, 1031-1041.

Blass, D. M. (2001). A conceptual framework for the interaction between psychiatry and religion. International Review of Psychiatry, 13, 79-85.

Bloch, P. M., Weitz, L. J., \& Abramowitz, S. I. (1980). Racial attribution effects on clinical judgment: A failure to replicate among White clinicians. American Journal of Community Psychology, 8, 485-493.

Boadella, D. (1998). Essence and ground: Towards the understanding of spirituality in psychotherapy. International Journal of Psychotherapy, 3, 29-51.

Booth, F. L. (1984). The gauntlet of spirituality. Alcoholism Treatment Quarterly, 1 139-141.

Bowen-Reid, T. L., \& Harrell, J. P. (2002). Racist experiences and health outcomes: An examination of spirituality as a buffer. Journal of Black Psychology, 28, $18-36$.

Boyer, P. (2001). Religion explained: The evolutionary origins of religious thought. New York: Basic Books. 
Burke, M. T., \& Hackney, H. (1999). Spirituality, religion, and CACREP curriculum standards. Journal of Counseling \& Development, 77, 251-257.

Burkhart, L., \& Solari-Twadell, A. (2001). Spirituality and religiousness: Differentiating the diagnoses through a review of the nursing literature. Nursing Diagnosis, 12, 45-54.

Burris, C. T. (1999). Religious Orientation Scale (Allport \& Ross, 1967). In P. C. Hill \& R. W. Hood (Eds.), Measures of religiosity (pp. 144-154). Birmingham, AL: Religious Education Press.

Cacioppo, J. T., Claiborn, C. D., Petty, R. E., \& Heesacker, M. (1991). A general framework for the study of attitude change in psychotherapy. In C. R. Snyder \& D. R. Forsyth (Eds.), Handbook of social and clinical psychology: A health perspective (pp. 523-539). Hillsdale, NJ: Erlbaum.

Carlson, T. D., Kirkpatrick, D., Hecker, L., \& Killmer, M. (2002). Religion, spirituality, and marriage and family therapy: A study of family therapists' beliefs about the appropriateness of addressing religious and spiritual issues in therapy. American Journal of Family Therapy, 30, 157-171.

Chackraborty, A. (1975). Ritual, a culture specific neurosis and obsessional states in Bengali culture. Indian Journal of Psychiatry, 17, 211-283.

Chae, M. H., Kelly, D. B., Brown, C. F., \& Bolden, M. A. (2004). Relationship of ethnic identity and spiritual development: An exploratory study. Counseling and Values, $49,15-26$. 
Chatters, L. M., Taylor, R. J., \& Lincoln, K. D. (1999). African American religious participation: A multi-sample comparison. Journal for the Scientific Study of Religion, 38, 132-145.

Clark, W. H. (1958). How do social scientists define religion? Journal of Social Psychology, 47, 143-147.

Cohen, J. (1988). Statistical power analysis for the behavioral sciences $\left(2^{\text {nd }}\right.$ ed.). Hillsdale, NJ: Erlbaum.

Collins, G. R. (1988). Christian counseling: A comprehensive guide (Rev. ed.). Dallas: Word.

Constantine, M. G. (2002). Racism attitudes, White racial identity attitudes, and multicultural counseling competence in school counselor trainees. Counselor Education and Supervision, 41, 162-174.

Constantine, M. G., Gloria, A. M., \& Ladany, N. (2002). The factor structure underlying three self-report multicultural competence scales. Cultural Diversity and Ethnic Minority Psychology, 8, 334-345.

Constantine, M. G., \& Ladany, N. (2000). Self-report multicultural counseling competence scales: Their relation to social desirability attitudes and multicultural case conceptualization ability. Journal of Counseling Psychology, 47, 155-164.

Constantine, M. G., Lewis, E. L., Conner, L. C., \& Sanchez, D. (2000). Addressing spiritual and religious issues in counseling African Americans: Implications for counselor training and practice. Counseling and Values, 45, 28-38. 
Cook, S. W., Borman, P. D., Moore, M. A., \& Kunkel, M. A. (2000). College students' perceptions of spiritual people and religious people. Journal of Psychology and Theology, 28, 125-137.

Cornett, C. (1998). The soul of psychotherapy: Recapturing the spiritual dimension in the therapeutic encounter. New York: The Free Press.

Davis, T. L., Kerr, B. A., \& Kurpius, S. E. (2003). Meaning, purpose, and religiosity in at-risk youth: The relationship between anxiety and spirituality. Journal of Psychology and Theology, 31, 356-365.

Dillman, D. A. (2007). Mail and internet surveys: The tailored design method ( $2^{\text {nd }}$ ed.). Hoboken, NJ: John Wiley \& Sons.

Dishion, T. J. (2000). Cross-setting consistency in early adolescent psychopathology: Deviant friendships and problem behavior sequalae. Journal of Personality, 68, 1109-1126.

Dobbins, R. D. (2000). Psychotherapy with Pentecostal Protestants. In P. S. Richards \& A. E. Bergin (Eds.), Handbook of psychotherapy and religious diversity (pp. 155-184). Washington, DC: American Psychological Association.

Dollahite, D. C. (1998). Fathering faith and spirituality. Journal of Men's Studies, 7, 3-15.

Donahue, M. J. (1985). Intrinsic and extrinsic religiousness: Review and metaanalysis. Journal of Personality and Social Psychology, 48, 400-419.

Donovan, J. E., \& Jessor, R. (1985). Structure of problem behavior in adolescence and young adulthood. Journal of Consulting and Clinical Psychology, 53, 890-904. 
Donovan, J. E., Jessor, R., \& Costa, F. M. (1988). Syndrome of problem behavior in adolescence: A replication. Journal of Consulting and Clinical Psychology, 56, 762765.

Doyle, D. (1992). Have we looked beyond the physical and psychosocial? Journal of Pain and Symptom Management, 7, 302-311.

D’Souza, R. (2002). Do patients expect psychiatrists to be interested in spiritual issues? Australasian Psychiatry, 10, 44-47.

Dyson, J., Cobb, M., \& Forman, D. (1997). The meaning of spirituality: A literature review. Journal of Advanced Nursing, 26, 1183-1188.

Eckhardt, C. I., Kassinove, H., \& Edwards, L. (1992). Religious beliefs and scientific ideology in psychologists: Conflicting or coexisting systems? Psychological Reports, 71, 131-145.

Eliassen, A. H., Taylor, J., \& Lloyd, D. A. (2005). Subjective religiousness and depression in the transition to adulthood. Journal for the Scientific Study of Religion, 44, 187-199.

Elkins, D. N., Hedstrom, L. J., Hughes, L. L., Leaf, J. A., \& Saunders, C. (1988). Toward a humanistic-phenomenological spirituality. Journal of Humanistic Psychology, $28,5-18$.

Ellis, A. (1980). Psychotherapy and atheistic values: A response to A. E. Bergin's "Psychotherapy and religious values." Journal of Consulting and Clinical Psychology, 48, 635-639. 
Emmons, R. A., \& Crumpler, C. A. (1999). Religion and spirituality?: The roles of sanctification and the concept of God. International Journal for the Psychology of Religion, 9, 17-24.

Erickson, M. J., Hecker, L., Kirkpatrick, D., Killmer, M., \& James, E. (2002). Clients' perceptions of marriage and family therapists addressing the religious and spiritual aspects of clients' lives: A pilot study. Journal of Family Psychotherapy, 13, 109-125.

Faul, F., Erdfelder, E., Lang, A.-G., \& Buchner, A. (2007). G*Power 3: A flexible statistical power analysis program for the social, behavioral, and biomedical sciences. Behavior Research Methods, 39, 175-191.

Fedio, P. (1986). Behavioral characteristics of patients with temporal lobe epilepsy. Psychiatric Clinics of North America, 9, 267-281.

Feeser, R. L. (1997). Counseling psychologist stance toward religion and spirituality as it relates to perceived attractiveness and overall prognosis of a religious, spiritual, or nonreligious client. Dissertation Abstracts International: Section B: The Sciences and Engineering, 58(10-B): 5641.

Ferriss, A. L. (2002). Religion and the quality of life. Journal of Happiness Studies, 3, 199-215.

Fitzgerald, W. V. (16 June 2010). Interview with Westboro Baptist Church: Hate in the name of God. DigitalJournal.com. Retrieved on June 20, 2010 from http://www.digitaljournal.com/article/293364.

Fontana, D. (1980). Some standardization for the Sandler-Hazari Obsessionality Inventory. British Journal of Medical Psychology, 53, 267-275. 
Fontana, D. (2003). Psychology, religion, and spirituality. Oxford: Blackwell.

Fox, J. (1991). Regression diagnostics. Thousand Oaks, CA: Sage.

Frame, M. W. (2003). Integrating religion and spirituality into counseling: A comprehensive approach. Pacific Grove, CA: Brooks/Cole.

Frame, R. E., Clarizio, H. F., Porter, A., \& Vinsonhaler, J. R. (1982).

Interclinician agreement and bias in school psychologists' diagnostic and treatment recommendations for a learning disabled child. Psychology in the Schools, 19, 319-327.

Franklin, D. L. (1985). Differential clinical assessments: The influence of class and race. Social Service Review, 59, 44-61.

Freud, S. (1907/1959). Obsessive actions and religious practices (Standard Edition, trans. By J. A. Stracey, Vol. 9, pp. 115-127). London: Hogarth.

Freud, S. (1927). The future of an illusion. In J. Strachey (Ed. And Trans.), The standard edition of the complete psychological works of Sigmund Freud (Vol. 21, pp. 558). London: Hogart Press, 1961.

Gallup, G., Jr. (1999). As nation observes national day of prayer, 9 in 10 pray - 3 in 4 daily. The Gallup Organization, May 6, www.gallup.com.

Gallup, G., Jr. (2001). Americans more religious now than ten years ago, but less so than in the 1950s and 1960s. The Gallup Organization, March 29, www.gallup.com.

Gallup News Service. (2001). Easter finds a religious nation. The Gallup Organization, April 13, www.gallup.com.

Gallup News Service. (2002). Poll topics and trends: Religion. The Gallup Organization, January 15, www.gallup.com. 
Garb, H. N. (1998). Studying the clinician: Judgment research and psychological assessment. Washington, DC: American Psychological Association.

Gartner, J. (1996). Religious commitment, mental health, and prosocial behavior: A review of the empirical literature. In E. P. Shafranske (Ed.), Religion and the Clinical Practice of Psychology (pp. 187-214). Washington, DC: American Psychological Association.

Gartner, J., Harmatz, M., Hohmann, A., Larson, D., \& Gartner, A. F. (1990a). The effect of client and counselor values on clinical judgment. Counseling and Values, 35, $58-62$.

Gartner, J., Harmatz, M., Hohmann, A., Larson, D., \& Gartner, A. F. (1990b). The effect of patient and clinician ideology on clinical judgment: A study of ideological countertransference. Psychotherapy: Theory, Research, Practice, Training, 27, 98-106.

Genia, V. (1993). A psychometric evaluation of the Allport-Ross I/E Scales in a religiously heterogeneous sample. Journal for the Scientific Study of Religion, 32, 284290.

Genia, V. (1994). Secular psychotherapists and religious clients: Professional considerations and recommendations. Journal of Counseling and Development, 72, 395398.

Gerson, J., Allen, R., Gold, J., \& Kose, G. (2000). Multiple belief systems in psychotherapy: The effects of religion and professional beliefs on clinical judgment. Journal of Contemporary Psychotherapy, 30, 27-32.

Gibson, W. C., \& Herron, W. G. (1990). Psychotherapists' religious beliefs and their perception of the psychotherapy process. Psychotherapy Reports, 66, 3-9. 
Gilbert, M. C. (2000). Spirituality in social work groups: Practitioners speak out. Social Work with Groups, 22, 67-84.

Gonzalez, A. (9 September 2010). Pastor nixes Quran-burning, claims NYC mosque deal. Yahoo! Associated Press (Gainesville, FL). Retrieved September 10, 2010 from http://news.yahoo.com/s/ap/20100909/ap_on_re_us/quran_burning.

Gorsuch, R. L. (1983). Factor analysis (2 ${ }^{\text {nd }}$ ed.). Hillsdale, NJ: Lawrence Erlbaum Associates.

Gorsuch, R. L., \& McFarland, S. G. (1972). Single vs. multiple-item scales for measuring religious values. Journal for the Scientific Study of Religion, 11, 53-64.

Gorsuch, R. L., \& McPherson, S. E. (1989). Intrinsic/extrinsic measurement: I/Erevised and single-item scales. Journal for the Scientific Study of Religion, 28, 348-354.

Graham, S. A. (1980). Psychotherapists' attitudes toward offender clients. Journal of Consulting and Clinical Psychology, 48, 796-797.

Green, S. B. (1991). How many subjects does it take to do a regression analysis? Multivariate Behavioral Research, 26, 499-510.

Greenberg, D. (1984). Are religious compulsions religious or compulsive: A phenomenological study. American Journal of Psychotherapy, 38, 524-532.

Greenberg, D., \& Shefler, G. (2002). Obsessive compulsive disorder in ultraorthodox Jewish patients: A comparison of religious and non-religious symptoms. Psychology and Psychotherapy, 75, 123-130.

Greenberg, D., \& Witztum, E. (1991). Problems in the treatment of religious patients. American Journal of Psychotherapy, 45, 554-565. 
Guinee, J. P., \& Tracey, T. J. G. (1997). Effects of religiousness and problem type on counselor description ratings. Journal of Counseling and Development, 76, 65-73.

Gushue, G. V., Constantine, M. G., \& Sciarra, D. T. (2008). The influence of culture, self-reported multicultural counseling competence, and shifting standards of judgment on perceptions of family functioning of white family counselors. Journal of Counseling and Development, 86, 85.

Haase, R. F., \& Ellis, M. V. (1987). Multivariate analyses of variance. Journal of Counseling Psychology, 34, 404-413.

Hall, G. C. N. (2001). Psychotherapy research with ethnic minorities: Empirical, ethical, and conceptual issues. Journal of Consulting and Clinical Psychology, 69, 502510.

Hall, T. W., \& Edwards, K. J. (1996). The initial development and factor analysis of the Spiritual Assessment Inventory. Journal of Psychology and Theology, 24, 233-246.

Harris, J. I., Schoneman, S. W., \& Carrera, S. R. (2002). Approaches to religiosity related to anxiety among college students. Mental Health, Religion, and Culture, 5, 253265.

Harris, K. A., Howell, D. S., Spurgeon, D. W., Sirken, A. W., McConnell, J. M., \& Coulter, D. R. (2006, August). Language games and the sacred: A definitional content analysis. Poster session presented at the American Psychological Association Convention 2006, New Orleans, LA.

Hawks, S. (1994). Spiritual health: Definition and theory. Wellness perspectives, $10,3-13$. 
Hecker, L. L., Trepper, T. S., Wetchler, J. L., \& Fontaine, K. L. (1995). The influence of therapist values, religiosity, and gender in the initial assessment of sexual addiction by family therapists. The American Journal of Family Therapy, 23, 261-272.

Heesacker, M., Conner, K. \& Prichard, S. (1995). Individual counseling and psychotherapy: Applications from the social psychology of attitude change. The Counseling Psychologist, 23, 611-632.

Heist, P., \& Yonge, G. (1968). Omnibus Personality Inventory: Form F manual. New York: The Psychological Corporation.

Heppner, P. P., Kivlighan, D. M., \& Wampold, B. E. (1999). Research design in counseling ( $2^{\text {nd }}$ ed.). Belmont, CA: Wadsworth.

Hermesh, H., Masser-Kavitsky, R., \& Gross-Isseroff, R. (2003). Obsessive compulsive disorder and Jewish religiosity. Journal of Nervous and Mental Disease, 191, 201-203.

Hickson, J., Housley, W., \& Wages, D. (2000). Counselors' perceptions of spirituality in the therapeutic process. Counseling and Values, 45, 58-66.

Higgins, N. C., \& Pollard, C. A. (1992). Relationship between religion-related factors and obsessive compulsive disorder. Current Psychology, 11, 79-85.

Hill, P. C., \& Hood, R. W. (Eds.). (1999). Measures of religiosity. Birmingham, AL: Religious Education Press.

Hill, T. \& Lewicki, P. (2007). Statistics: Methods and applications. StatSoft: Tulsa, OK.

Hill, P. C., Pargament, K. I., Hood, R. W. Jr., McCullough, M. E., Swyers, J. P., Larson, D. B., \& Zinnbauer, B. J. (2000). Conceptualizing religion and spirituality: Points 
of commonality, points of departure. Journal for the Theory of Social Behaviour, 30, 5177.

Hillowe, B. V. (1985). The effect of religiousness of therapist and patient on clinical judgment. Unpublished doctoral dissertation, Adelphi University.

Hoge, D. R. (1996). Religion in America: The demographics of belief and affiliation. In E. P. Shafranske (Ed.), Religion and the clinical practice of psychology (pp. 21-41). Washington, D.C.: American Psychological Association.

Holcomb-McCoy, C. C. (2000). Multicultural counseling competencies: An exploratory factor analysis. Journal of Multicultural Counseling and Development, 28, 83-97.

Hood, R. W., Jr. (1975). The construction and preliminary validation of a measure of reported spiritual experience. Journal for the Scientific Study of Religion, 14, 29-41.

Hopping, M. (1984). Psychic seizures: A diagnostic challenge. Bulletin of the Menninger Clinic, 48, 401-417.

Houts, A. C., \& Graham, K. (1986). Can religion make you crazy?: Impact of client and therapist religious values on clinical judgments. Journal of Consulting and Clinical Psychology, 54, 267-271.

Ingersoll, R. E. (1994). Spirituality, religion, and counseling: Dimensions and relationships. Counseling and Values, 38, 98-112.

Ingersoll-Dayton, B., Krause, N., \& Morgan, D. (2002). Religious trajectories and transitions over the life course. International Journal of Aging and Human Development, $55,51-70$. 
Jaccard, J., \& Turrisi, R. (1990). Interaction effects in multiple regression. Thousand Oaks, CA: Sage.

Jackson, M. L. (1995). Multicultural counseling: Historical perspectives. In J. G. Ponterotto, J. M. Casas, L. A. Suzuki, \& C. M. Alexander (Eds.), Handbook of multicultural counseling (pp. 3-16). Thousand Oaks, CA: Sage.

James, W. (1961). The varieties of religious experience. New York: Collier Books. (Original work published 1902)

Jones, C. H. (1991). The effect of religious doubt on clinical judgment. Unpublished doctoral dissertation, Spalding University.

Judd, C. M., \& McClelland, G. H. (1989). Data analysis: A model comparison approach. San Diego, CA: Harcourt, Brace, Jovanovich.

Juni, S. \& Fischer, R. E. (1985). Religiosity and preoedipal fixation. Journal of Genetic Psychology, 146, 27-35.

Kahle, P. A. (1998). The influence of the person of the therapist on the integration of spirituality and psychotherapy. Dissertation Abstracts International: Section B: The Sciences and Engineering, 58(8-B): 4453.

Kahle, P. A., \& Robbins, J. M. (2004). The power of spirituality in therapy: Integrating spiritual and religious beliefs in mental health practice. New York: The Haworth Pastoral Press.

Kahneman, D., \& Tversky, A. (1973). On the psychology of prediction. Psychological Review, 80, 237-251. 
Kahoe, R. D. (1974). Personality and achievement correlates of intrinsic and extrinsic religious orientations. Journal of Personality and Social Psychology, 29, 812818.

Kaplan, H. B., \& Johnson, R. J. (2001). Social deviance: Testing a general theory. New York: Kluwer Academic/Plenum Publishers.

Kass, J. D., Friedman, R., Lesserman, J., Zuttermeister, P., \& Benson, H. (1991). Health outcomes and a new index of spiritual experience. Journal for the Scientific Study of Religion, 30, 203-211.

Kassinove, H., \& Uecke, C. I. (1991). Religious involvement and behavior therapy training: Student conflicts and ethical concerns. The Behavior Therapist, 14, 148150.

Keller, R. R. (2000). Religious diversity in North America. In P. S. Richards \& A. E. Bergin (Eds.), Handbook of psychotherapy and religious diversity (pp. 27-56). Washington, DC: American Psychological Association.

Kelly, E. W., Jr. (1995). Spirituality and religion in counseling and psychotherapy: Diversity in theory and practice. Alexandria, VA: American Counseling Association.

Kendler, K. S., Liu, X., Gardner, C. O., McCullough, M. E., Larson, D., \& Prescott, C. A. (2003). Dimensions of religiosity and their relationship to lifetime psychiatric and substance use disorders. American Journal of Psychiatry, 160, 496-503.

Khalili, S., Murken, S., Reich, K. H., Ali Shah, A., \& Vahabzadeh, A. (2002). Religion and mental health in cultural perspective: Observations and reflections after the 
first international congress on religion and mental health, Tehran, 16-19 April 2001. The International Journal for the Psychology of Religion, 12, 217-237.

Kirkpatrick, L. A. (1989). A psychometric analysis of the Allport-Ross and Feagin measures of intrinsic-extrinsic religious orientation. In D. O. Moberg \& M. L. Lynn (Eds.), Research in the social scientific study of religion, Vol. 1 (pp. 1-31). Greenwich, CT: JAI Press.

Kiselica, M. S. (1998). Preparing Anglos for the challenges and joys of multiculturalism. The Counseling Psychologist, 26, 5-21.

Kivley, L. R. (1986). Therapist attitude toward including religious issues in therapy. Journal of Psychology and Christianity, 5, 37-45.

Klineman, G., Butler, S., \& Conn, D. (1980). The cult that died. New York: Putnam.

Knudsen, H. C., \& Bolwig, T. G. (1986). The relationship between epilepsy and personality: Reality or myth. Nordisk Psykiatrisk Tidsskrift, 40, 337-343.

Kocarek, C. E., Talbot, D. M., Batka, J. C., \& Anderson, M. Z. (2001). Reliability and validity of three measures of multicultural competency. Journal of Counseling and Development, 79, 486-496.

Koenig, H. G. (1998). Handbook of religion and mental health. San Diego: Academic Press.

Koenig, H. (2005). Faith and mental health. Philadelphia: Templeton Foundation Press. 
Koenig, H. G., Ford, S. M., George, L. K., Blazer, D. G., \& Meador, K. G. (1993). Religion and anxiety disorder: An examination and comparison of associations in young, middle-aged, and elderly adults. Journal of Anxiety Disorders, 7, 321-342.

Koenig, H. G., George, L. K., Blazer, D. G., Pritchett, J. T., \& Meador, K. G. (1993). The relationship between religion and anxiety in a sample of communitydwelling older adults. Journal of Geriatric Psychiatry, 26, 65-93.

Koenig, H. G., George, L. K., Meador, K. G., Blazer, D. G., \& Dyck P. B. (1994). Religious affiliation and psychiatric disorder among Protestant baby boomers. Hospital and Community Psychiatry, 45, 586-596.

Koocher, G., \& Keith-Spiegel, P. (1998). Ethics in psychology: Professional standards and cases ( $2^{\text {nd }} e d$.). Oxford, NY: Oxford University Press.

Krathwohl, D. R. (1998). Methods of educational and social science research: An integrated approach ( $2^{\text {nd }}$ ed.). New York: Addison-Wesley.

Lalich, J. (2004). Bounded choice. Berkeley: University of California Press.

Lannert, J. L. (1992). Spiritual and religious attitudes, beliefs, and practices of clinical training directors and their internship sites. Unpublished doctoral dissertation, University of Southern California.

Lee, R. R. (1965). Theological belief as a dimension of personality. Unpublished doctoral dissertation, Northwestern University, Evanston, IL.

Legere, T. E. (1984). A spirituality for today. Studies in Formative Spirituality, 5, $375-383$. 
Levin, J. S., Taylor, R. J., \& Chatters, L. M. (1994). Race and gender differences in religiousness among older adults: Findings from four national surveys. Journal of Gerontology: Social Sciences, 49, S137-S145.

Lewis, C. A. (1994). Religiosity and obsessionality: The relationship between Freud's “religious practices.” Journal of Psychology, 128, 189-196.

Lewis, C. A. (1998). Cleanliness is next to Godliness: Religiousness and obsessiveness. Journal of Religion and Health, 37, 49-62.

Lewis, C. A., \& Joseph, J. (1994a). Religiosity: Psychoticism and obsessionality in Northern Irish university students. Personality and Individual Differences, 17, 685687.

Lewis, C. A., \& Joseph, S. (1994b). Obsessive actions and religious practices. Journal of Psychology, 128, 699-700.

Lewis, C. A., \& Maltby, J. (1992). Religiosity and preoedipal fixation: A refinement. Journal of Psychology, 126, 687-688.

Lewis, C. A., \& Maltby, J. (1994). Religious attitudes and obsessional personality traits among UK adults. Psychological Reports, 75, 353-356.

Lewis, C. A., \& Maltby, J. (1995). Religious attitude and practice: The relationship with obsessionality. Personality and Individual Differences, 19, 105-108.

Lewis, G., Croft-Jeffreys, C., \& David, A. (1990). Are British psychiatrists racist? British Journal of Psychiatry, 157, 410-415.

Lewis, K. N., \& Lewis, D. A. (1985). Impact of religious affiliation on therapists' judgments of patients. Journal of Consulting and Clinical Psychology, 53, 926-932. 
Lindenthal, J., Myers, J., Pepper, M., \& Stern, M. (1970). Mental status and religious behavior. Journal for the Scientific Study of Religion, 9, 143-149.

Lindgren, K. N., \& Coursey, R. D. (1995). Spirituality and serious mental illness: A two-part study. Psychosocial Rehabilitation Journal, 18, 93-111.

Lindsey, K. P., \& Paul, G. L. (1989). Involuntary commitments to public mental institutions: Issues involving the overrepresentation of Blacks and assessment of relevant functioning. Psychological Bulletin, 106, 171-183.

Lodge, M. (1981). Magnitude scaling: Qualitative measurement of options. Sage University Paper series on Quantitative Applications in the Social Sciences, series no. 07001. Beverly Hills and London: Sage.

Long, T. E., \& Hadden, J. K. (1983). Religious conversion and the concept of socialization: Integrating the brainwashing and drift models. Journal for the Scientific Study of Religion, 22, 1-14.

Lopez, S. R. (1989). Patient variable biases in clinical judgment: Conceptual overview and methodological considerations. Psychological Bulletin, 106, 184-203.

Lunneborg, C. E., \& Abbott, R. D. (1983). Elementary multivariate analysis for the behavioral sciences. New York: North-Holland.

Lutz, J. G., \& Eckert, T. L. (1994). The relationship between canonical correlation analysis and multivariate multiple regression. Educational and Psychological Measurement, 54, 666-675.

Makarec, R., \& Persinger, M.A. (1985). Temporal lobe signs: Electroencephalographic validity and enhanced scores in special populations. Perceptual and Motor Skills, 60, 831-842. 
Maltby, J., McCollam, P., \& Millar, D. (1994). Religiosity and obsessionality: A refinement. Journal of Psychology, 128, 609-611.

Manese, J. E., Wu, J. T., \& Nepomuceno, C. A. (2001). The effect of training on multicultural counseling competencies: An exploratory study over a ten-year period. Journal of Multicultural Counseling and Development, 29, 31-40.

Marcinkowski, P. L. (1993). Verbal patterns, clinical impressions, and pathology ascriptions relative to theistic content, locus of evaluation, and degree of theism. Unpublished doctoral dissertation, California School of Professional Psychology.

Markstrom, C. (1999). Religious involvement and adolescent psychosocial development. Journal of Adolescence, 22, 205-221.

Martinez, F. I. (1991). Therapist-client convergence and similarity of religious values: Their effect on client improvement. Journal of Psychology and Christianity, 10, 137-143.

Mataix-Cols, D., Rosario-Campos, M. C., \& Leckman, J. F. (2002). A multidimensional model of obsessive-compulsive disorder. American Journal of Psychiatry, 162, 228-238.

Mays, V. M., Caldwell, C. H., \& Jackson, J. S. (1996). Mental health symptoms and service utilization patterns of help-seeking among African American women. In H. W. Neighbors \& J. S. Jackson (Eds.), Mental health in Black America (pp. 161-176). Thousand Oaks, CA: Sage.

McCullough, M. E., \& Larson, D. B. (1998). Future directions in research. In H. G. Koenig (Ed.), Handbook of religion and mental health (pp. 96-107). San Diego: Academic Press. 
McCullough, M. E., Weaver, A. J., Larson, D. B., \& Aay, K. R. (2000).

Psychotherapy with mainline Protestants: Lutheran, Presbyterian, Episcopal/Anglican, and Methodist. In P. S. Richards \& A. E. Bergin (Eds.), Handbook of psychotherapy and religious diversity (pp. 105-129). Washington, DC: American Psychological Association.

McGee, L., \& Newcomb, M. D. (1992). General deviance syndrome: Expanded hierarchical evaluations at four ages from early adolescence to adulthood. Journal of Consulting and Clinical Psychology, 60, 766-776.

McKinnon, A. M. (2002). Sociological definitions, language games, and the “essence" of religion. Method and theory in the study of religion, 14, pp. 61-83.

McLatchie, L. R., \& Draguns, J. G. (1984). Mental health concepts of evangelical Protestants. Journal of Psychology, 118, 147-159.

McLaughlin, M., \& Balch, P. (1980). Effects of client-therapist ethnic homophily on therapists' judgments. American Journal of Community Psychology, 8, 243-252.

McLennan, N. A., Rochow, S., \& Arthur, N. (2001). Religious and spiritual diversity in counselling. Guidance \& Counseling, 16, 132-137.

Miller, L., \& Kelley, B. S. (2005). Relationships of religiousness and spirituality with mental health and psychopathology. In R. F. Paloutzian \& C. L. Park (Eds.), Handbook of the psychology of religion and spirituality (pp. 460-478). New York: Guilford.

Minyard, S. E. (1983). An idiographic investigation of clinical judgment: Clinicians' use of religious "cues” in the judgment of psychological adjustment. Unpublished doctoral dissertation, Bowling Green State University. 
Moberg, D. O. (1984). Subjective measures of spiritual well-being. Review of Religious Research, 25, 351-359.

Momen, M. (1999). The phenomenon of religion: A thematic approach. Oxford: Oneworld.

Moore, K. A., \& Glei, D. (1995). Take the plunge: An examination of positive youth development. Journal of Adolescent Research, 10, 15-40.

Moriarty, M. G. (1992). The new charismatics: A concerned voice responds to dangerous new trends. Grand Rapids, MI: Zondervan Publishing House.

Morrow, K. A., \& Deidan, C. T. (1992). Bias in the counseling process: How to recognize and avoid it. Journal of Counseling and Development, 70, 571-577.

Morrow, D., Worthington, E. L., \& McCullough, M. E. (1993). Observers' perceptions of a counselor's treatment of a religious issue. Journal of Counseling and Development, 71, 452-456.

Mukherjee, S., Shukla, S., Woodle, J., Rosen, A. M., \& Olarte, S. (1983). Misdiagnosis of schizophrenia in bipolar patients: A multiethnic comparison. American Journal of Psychiatry, 140, 1571-1574.

Nalven, F. B., Hofmann, L. J., \& Bierbryer, B. (1969). The effects of subjects' age, sex, race, and socioeconomic status on psychologists' estimates of "true IQ" from WISC scores. Journal of Clinical Psychology, 25, 271-274.

Newcomb, M. D., \& Burns Loeb, T. (1999). Poor parenting as an adult problem behavior: General deviance, deviant attitudes, inadequate family support and bonding, or just bad parents? Journal of Family Psychology, 13, 175-193. 
Newcomb, M. D., \& McGee, L. (1991). Influence of sensation seeking on general deviance and specific problem behaviors from adolescence to young adulthood. Journal of Personality and Social Psychology, 61, 614-628.

Nisbett, R., \& Ross, L. (1980). Human inference: Strategies and shortcomings of social judgment. Englewood Cliffs, NJ: Prentice Hall.

Nobody, I. M. (2000). Just seeing if you're paying attention. Not a Real Journal, $10,55-56$.

O’Collins, G., \& Farrugia, E. G. (1991). A concise dictionary of theology. New York: Paulist Press.

O’Connor, S., \& Vandenberg, B. (2005). Psychosis or faith?: Clinicians' assessment of religious beliefs. Journal of Consulting and Clinical Psychology, 73, 610616.

Okasha, A., Lotaief, F., Ashour, A. M., El Mahalawy, N., Seif El Dawla, A., \& El-Kholy, G. (2000). The prevalence of obsessive compulsive symptoms in a sample of Egyptian psychiatric patients. Encephale, 26(4), 1-10.

O’Malley, M. N., Gearhart, R., \& Becker, L. A. (1984). On cooperation between psychology and religion: An attitudinal survey of therapists and clergy. Counseling and Values, 28, 117-121.

Oman, D., \& Thoresen, C. E. (2005). Do religion and spirituality influence health? In R. F. Paloutzian \& C. L. Park (Eds.), Handbook of the psychology of religion and spirituality (pp. 435-459). New York: Guilford. 
Osgood, D. W., Johnston, L. D., O’Malley, P. M., \& Bachman, J. G. (1988). The generality of deviance in late adolescence and early adulthood. American Sociological Review, 53, 81-93.

Paloutzian, R. F., \& Park, C. L. (Eds.). (2005). Handbook of the psychology of religion and spirituality. New York: Guilford.

Pardini, D. A., Plante, T. G., Sherman, A., \& Stump, J. E. (2000). Religious faith and spirituality in substance abuse recovery: Determining the mental health benefits. Journal of Substance Abuse Treatment, 19, 347-354.

Pate, R. H., Jr., \& Bondi, A. M. (1992). Religious beliefs and practice: An integral aspect of multicultural awareness. Counselor Education and Supervision, 32, 108-115.

Pavkov, T. W., Lewis, D. A., \& Lyons, J. S. (1989). Psychiatric diagnoses and racial bias: An empirical investigation. Professional Psychology, 20, 364-368.

Pederson, P. (1990). The multicultural perspective as a fourth force in counseling. Journal of Mental Health Counseling, 12, 93-95.

Pedhazur, E. J. (1997). Multiple regression in behavioral research: Explanation and prediction $\left(3^{\mathrm{rd}}\right.$ ed.). Toronto: Thomson Learning.

Perkins, H. W. (1992). Student religiousness and social justice concerns in England and the United States: Are they still related? Journal for the Scientific Study of Religion, 31, 353-360.

Persinger, M. A. (1984). People who report religious experiences may also display enhanced temporal-lobe signs. Perceptual and Motor Skills, 58, 963-975. 
Persinger, M. A., \& Makarec, R. (1987). Temporal lobe epileptic signs and correlative behaviors displayed by normal populations. Journal of General Psychology, 114, 179-195.

Peteet, J. R. (1994). Approaching spiritual problems in psychotherapy: A conceptual framework. Journal of Psychotherapy Practice and Research, 3, 237-245.

Peterson, G. A. (2001). Spiritual - Religious Orientation Scale: Initial psychometric analysis. Unpublished doctoral dissertation, California Institute of Integral Studies.

Piedmont, R. L. (2005). The role of personality in understanding religious and spiritual constructs. In R. F. Paloutzian \& C. L. Park (Eds.), Handbook of the psychology of religion and spirituality (pp. 253-273). New York: Guilford.

Piedmont, R. L., Ciarrocchi, J. W., Dy-Liacco, G., Mapa, A. T., \& Williams, J. E. G. (2003). An evaluation of spirituality and religiosity as empirical constructs for personality research. Manuscript under review.

Pfeifer, S., \& Waelty, U. (2000). Anxiety, depression, and religiosity: A controlled clinical study. Mental Health, Religion, and Culture, 2(1), 35-45.

Ponterotto, J. G., Casas, J. M., Suzuki, L. A., \& Alexander, C. M. (1995). Handbook of multicultural counseling. Thousand Oaks, CA: Sage.

Ponterotto, J. G., Casas, J. M., Suzuki, L. A., \& Alexander, C. M. (2001). Handbook of multicultural counseling ( $2^{\text {nd }}$ ed.). Thousand Oaks, CA: Sage.

Ponterotto, J. G., Fuertes, J. N., \& Chen, E. C. (2000). Models of multicultural counseling. In S. D. Brown \& R. W. Lent (Eds.), Handbook of counseling psychology ( $3^{\text {rd }}$ ed., pp. 639-669). New York: John Wiley. 
Ponterotto, J. G., Gretchen, D., Utsey, S. O., Rieger, B. P., \& Austin, R. (2002). A revision of the Multicultural Couneling Awareness Scale (MCAS). Journal of Multicultural Counseling and Development, 30, 153-180.

Ponterotto, J. G., \& Potere, J. C. (2003). The Multicultural Counseling Knowledge and Awareness Scale (MCKAS). In D. B. Pope-Davis, H. L. K. Coleman, W. M. Liu, \& R. L. Toporek (Eds.), Handbook of multicultural competencies in counseling and psychology (pp. 137-153). Thousand Oaks, CA: Sage Publications.

Ponterotto, J. G., Rieger, B. T., Barrett, A., Harris, G., Sparks, R., Sanchez, C. M., et al. (1996). Development and initial validation of the Multicultural Counseling Awareness Scale. In G. R. Sodowsky \& J. C. Impara (Eds.), Multicultural assessment in counseling and clinical psychology (pp. 247-282). Lincoln, NE: Buros Institute of Mental Measurements.

Ponterotto, J. G., Sanchez, C. M., \& Magids, D. (1991, August). Initial development of the Multicultural Counseling Awareness Scale. Paper presented at the annual meeting of the American Psychological Association, San Francisco.

Powers, R. L. (2003). Robert L. Powers's original contribution to "Spirituality in the Adlerian forum.” Journal of Individual Psychology, 59, 84-85.

Pressman, P., Lyons, J. S., Larson, D. B., \& Gartner, J. (1992). Religion, anxiety, and the fear of death. In J. F. Schumaker (Ed.), Religion and mental health (pp. 98-109). New York: Oxford University Press.

Quackenbos, S., Privette, G., \& Kleinz, B. (1985). Psychology: Sacred or secular? Journal of Counseling and Development, 63, 290-293. 
Rayburn, C. A. (2000). Psychotherapy with Seventh-Day Adventists. In P. S.

Richards \& A. E. Bergin (Eds.), Handbook of psychotherapy and religious diversity (pp. 211-234). Washington, DC: American Psychological Association.

Resnicow, K., Braithwaite, R. L., \& Kuo, J. A. (1997). Interpersonal interventions for minority adolescents. In D. K. Wilson \& W. C. Taylor (Eds.), Health-promoting and health-compromising behaviors among minority adolescents (pp. 201-228). Washington, DC: American Psychological Association.

Richards, P. S., \& Bergin, A. E. (Eds.). (2000). Handbook of psychotherapy and religious diversity. Washington, DC: American Psychological Association.

Richards, P. S., \& Potts, R. W. (1995). Using spiritual interventions in psychotherapy: practices, successes, failures, and ethical concerns of Mormon psychotherapists. Professional Psychology: Research and Practice, 26, 163-170.

Richardson, J. T. (1992). Mental health of cult consumers: Legal and scientific controversy. (pp. 233-244). In J. F. Schumaker (Ed.), Religion and mental health. New York: Oxford.

Richeson, J. A., \& Nussbaum, R. J. (2004). The impact of multiculturalism versus color-blindness on racial bias. Journal of Experimental Social Psychology, 40, 417-423.

Rodgers, J. L. (2010). The epistemology of mathematical and statistical modeling. American Psychologist, 65, 1-12.

Rose, E. M., Westefeld, J. S., \& Ansely, T. N. (2001). Spiritual issues in counseling: Clients' beliefs and preferences. Journal of Counseling Psychology, 48, 6171. 
Ross, C. E. (1990). Religion and psychological distress. Journal for the Scientific Study of Religion, 29, 236-245.

Salovey, P., \& Turk, D. C. (1991). Clinical judgment and decision-making. In C. R. Snyder \& D. R. Forsyth (Eds.), Handbook of social and clinical psychology: The health perspective (pp. 416-437). New York: Pergamon Press.

Sanderson, S., Vandenberg, B., \& Paese, P. (1999). Authentic religious experience or insanity? Journal of Clinical Psychology, 55, 607-616.

Saroglou, V. (2002). Religiousness, religious fundamentalism, and quest as predictors of humor creation. International Journal for the Psychology of Religion, 12, $177-188$.

Sattler, J. M., \& Kuncik, T. M. (1976). Ethnicity, socioeconomic status, and pattern of WISC scores as variables that affect psychologists" estimates of "effective intelligence." Journal of Clinical Psychology, 32, 362-366.

Schwartz, J. M., \& Abramowitz, S. I. (1978). Effects of female client physical attractiveness on clinical judgment. Psychotherapy: Theory, Research, and Practice, 15, 251-257.

Schnittker, J. (2001). When is faith enough?: The effects of religious involvement on depression. Journal for the Scientific Study of Religion, 40, 393-411.

Segal, S. P., Bola, J. R., \& Watson, M. A. (1996). Race, quality of care, and antipsychotic prescribing practices in psychiatric emergency services. Psychiatric Services, 47, 282-286.

Seligman, M. R. (1968). The interracial casework relationship. Smith College Studies in Social Work, 39, 84. 
Sell, K. L., \& Goldsmith, W. M. (1988). Concerns about professional counseling: An exploration of five factors and the role of Christian orthodoxy. Journal of Psychology and Christianity, 7, 5-21.

Sethi, S., \& Seligman, M. E. P. (1991). Optimism and fundamentalism. Psychological Science, 4, 256-259.

Seyfarth, L. H., Larsen, K. S., Lamont, K., Haasch, C., Hale, T., \& Haskin, D. (1984). Attitude toward evangelism: Scale development and validity. Journal of Social Psychology, 123, 55-61.

Shafranske, E. P. (1996). Religious beliefs, affiliations, and practices of clinical psychologists. In E. P. Shafranske (Ed.), Religion and the clinical practice of psychology (pp. 149-162). Washington, D.C.: American Psychological Association.

Shafranske, E. P. (2000). Psychotherapy with Roman Catholics. In P. S. Richards \& A. E. Bergin (Eds.), Handbook of psychotherapy and religious diversity (pp. 105-129). Washington, DC: American Psychological Association.

Shafranske, E. P. (2005). The psychology of religion in clinical and counseling psychology. In R. F. Paloutzian \& C. L. Park (Eds.), Handbook of the psychology of religion and spirituality (pp. 496-514). New York: Guilford.

Shafranske, E. P., \& Gorsuch, R. L. (1984). Factors associated with the perception of spirituality in psychotherapy. Journal of Transpersonal Psychology, 16, 231-241.

Shafranske, E. P., \& Malony, H. N. (1990). Clinical psychologists' religious and spiritual orientations and their practice of psychotherapy. Psychotherapy, 27, 72-78.

Sigmund, J. A. (2002). Religion, spirituality, and spiritualism. American Journal of Psychiatry, 159, 2117-2118. 
Simon, R. J., Fleiss, J. L., Gurland, B. J., Stiller, P. R., \& Sharpe, L. (1973). Depression and schizophrenia in hospitalized Black and White mental patients. Archives of General Psychiatry, 28, 509-512.

Skinner, B. F. (1953). Science and human behavior. New York: Macmillan.

Smith, T. B., McCullough, M. E., \& Poll, J. (2003). Religiousness and depression: Evidence for a main effect and the moderating influence of stressful life events. Psychological Bulletin, 129, 614-636.

Sorensen, A. E., \& Bolwig, T. G. (1987). Personality and epilepsy: New evidence for a relationship? A review. Comprehensive Psychiatry, 28, 369-383.

Spence, J. T., Cotton, J. W., Underwood, B. J., \& Duncan, C. P. (1992). Elementary statistics (Rev. $5^{\text {th }}$ ed.). Englewood Cliffs, NJ: Prentice Hall.

Spengler, P. M., Blustein, D. L., \& Strohmer, D. C. (1990). Diagnostic and treatment overshadowing of vocational problems by personal problems. Journal of Counseling Psychology, 37, 372-381.

Spengler, P. M., \& Strohmer, D. C. (1994). Clinical judgment biases: The moderating roles of counselor cognitive complexity and counselor client preferences. Journal of Counseling Psychology, 41, 8-17.

Spengler, P. M., Strohmer, D. C., Dixon, D. N., \& Shivy, V. A. (1995). A scientist-practitioner model of psychological assessment: Implications for training, practice, and research. The Counseling Psychologist, 23, 506-534.

Spitzer, R., Skodol, A., Gibbon, M., \& Williams, J. (1981). DSM-III casebook. Washington, DC: American Psychiatric Association. 
Stanard, R. P., Sandhu, D. S., \& Painter, L. C. (2000). Assessment of spirituality in counseling. Journal of Counseling and Development, 78, 204-210.

Stark, R., \& Bainbridge, W. S. (1996). Religion, deviance, and social control. New York: Routledge.

Steketee, G., Quay, S., \& White, K. (1991). Religion and guilt in OCD patients. Journal of Anxiety Disorders, 5, 359-367.

Stevens, G. (1981). Bias in the attribution of hyperkinetic behavior as a function of ethnic identification and socioeconomic status. Psychology in the Schools, 18, 99-106.

Stevens, J. (1986). Applied multivariate statistics for the social sciences. Hillsdale, NJ: Erlbaum.

Stevens, J. P. (2009). Multivariate regression (pp. 128-131). Applied multivariate statistics for the social sciences $\left(5^{\text {th }}\right.$ ed.). New York: Routledge.

St. George, A., \& McNamara, P. H. (1984). Religion, race, and psychological well-being. Journal for the Scientific Study of Religion, 23, 351-363.

Strickland, B. R., \& Wendell, S. C. (1972). Religious orientation, racial prejudice, and dogmatism: A study of Baptists and Unitarians. Journal for the Scientific Study of Religion, 11, 395-399.

Strickland, T. L., Jenkins, J. O., Myers, H. F., \& Adams, H. E. (1988). Diagnostic judgments as a function of client and therapist race. Journal of Psychopathology and Behavioral Assessment, 10, 141-151.

Sue, D. W., Bernier, J. E., Durran, A., Feinberg, L., Pederson, P., Smith, E. J., \& Vasquez-Nuttall, E. (1982). Position paper: Cross-cultural counseling competencies. The Counseling Psychologist, 10, 45-52. 
Sue, D. W., Arredondo, P., \& McDavis, R. J. (1992). Multicultural counseling competencies and standards: A call to the profession. Journal of Counseling and Development, 70, 477-486; Journal of Multicultural Counseling and Development, 20, 64-89.

Szasz, T. (1970). Ideology and insanity: Essays on the psychiatric dehumanization of man. New York: Doubleday Anchor.

Tabachnick, B. G., \& Fidell, L. S. (2001). Using multivariate statistics (4 ${ }^{\text {th }}$ ed.). Boston: Allyn and Bacon.

Tanenhaus, J., \& Murphy, W. (1981). Patterns of public support for the Supreme Court: A panel study. Journal of Politics, 43, 324-339.

Tart, C. (1975). Introduction. In C. T. Tart (Ed.), Transpersonal psychologies (pp. 3-7). New York: Harper \& Row.

Taylor, R. J., Chatters, L. M., Jayakody, R., \& Levin, J. S. (1996). Black and White differences in religious participation: A multisample comparison. Journal for the Scientific Study of Religion, 35, 403-410.

Tek, C., \& Ulug, B. (2001). Religiousness and religious obsessions in obsessivecompulsive disorder. Psychiatry Research, 104, 99-108.

Thalheimer, W., \& Cook, S. (2002, August). How to calculate effect sizes from published research: A simplified methodology. Retrieved August 21, 2010 from www.work-learning.com/effect_sizes.htm.

Thompson, A. D. (1974). Open-mindedness and indiscrinate anti-religious orientation. Journal for the Scientific Study of Religion, 13, 471-477. 
Thurston, N. S. (2000). Psychotherapy with evangelical and fundamentalist Protestants. In P. S. Richards \& A. E. Bergin (Eds.), Handbook of psychotherapy and religious diversity (pp. 131-153). Washington, DC: American Psychological Association.

Turner, R. P., Lukoff, D., Barnhouse, R. L., \& Lu, B. G. (1995). Religious or spiritual problem: A culturally sensitive category in the DSM-IV. Journal of Nervous and Mental Disease, 183, 438-444.

Umbenhauer, S. L., \& DeWitte, L. L. (1978). Patient race and social class: Attitudes and decisions among three groups of mental health professionals. Comprehensive Psychiatry, 19, 509-515.

Vaughan, F. (1991). Spiritual issues in psychotherapy. Journal of Transpersonal Psychology, 23, 105-119.

Veroff, J., Kulka, R. A., \& Douvan, E. (1981). Mental health in America. New York: Basic Books.

Wadsworth, R. D., \& Checketts, K. T. (1980). Influence of religious affiliation on psychodiagnosis. Journal of Consulting and Clinical Psychology, 48, 234-240.

Walker, B. S. (1999). The diagnosis and treatment of major depression in AIDS patients: Effect of counselor experience and attitude toward people with AIDS. Unpublished doctoral dissertation, Ball State University.

Walker, D. F., Gorsuch, R. L., \& Tan, S. Y. (2004). Therapists' integration of religion and spirituality in counseling: A meta analysis. Counseling and Values, 49, 6980.

Warner, R. (1979). Racial and sexual bias in psychiatric diagnosis. Journal of Nervous and Mental Disease, 167, 303-310. 
Watson, P. J., Hood, R. W., Jr., Morris, R. J., \& Hall, J. R. (1987). The relationship between religiosity and narcissism. Counseling and Values, 31, 179-184.

Watson, P. J., Morris, R. J., \& Hood, R. W., Jr. (1988a). Sin and self-functioning, Part 1: Grace, guilt, and self-consciousness. Journal of Psychology and Theology, 16, 254-268.

Watson, P. J., Morris, R. J., \& Hood, R. W., Jr. (1988b). Sin and self-functioning, Part 2: Grace, guilt, and psychological adjustment. Journal of Psychology and Theology, $16,270-281$.

Watson, P. J., Morris, R. J., \& Hood, R. W., Jr. (1988c). Sin and self-functioning, Part 3: The psychology and ideology of irrational beliefs. Journal of Psychology and Theology, 16, 348-361.

Wink, P., \& Scott, J. (2005). Does religiousness buffer against the fear of death and dying in late adulthood?: Findings from a longitudinal study. Journals of Gerontology Series B: Psychological Sciences and Social Sciences, 60B, 207-214.

Wolf, C. T., \& Stevens, P. (2001). Integrating religion and spirituality in marriage and family counseling. Counseling and Values, 46, 66-75.

Worthington, E. L. (1986). Religious counseling: A review of published empirical research. Journal of Counseling and Development, 64, 421-431.

Worthington, E. L., Kurusu, T. A., McCullough, M. E., \& Sandage, S. J. (1996). Empirical research on religion and psychotherapeutic processes and outcomes: A 10-year review and research prospectus. Psychological Bulletin, 119, 448-487. 
Worthington, E. L., \& Scott, G. G. (1983). Goal selection for counseling with potentially religious clients by professional and student counselors in explicitly Christian or secular settings. Journal of Psychology and Theology, 11, 318-329.

Wulff, D. M. (1996). The psychology of religion: An overview. In E. P. Shafranske (Ed.), Religion and the clinical practice of psychology. Washington, DC: American Psychological Association.

Yalom, I. D. (1980). Existential psychotherapy. New York: Basic Books. Yaryura-Tobias, J. A., Grunes, M. S., Todaro, J., McKay, D., Neziroglu, F. A., \& Stockman, R. (2000). Nosological insertion of axis I disorders in the etiology of obsessive-compulsive disorder. Journal of Anxiety Disorders, 14, 19-30.

Yossifova, M., \& Loewenthal, K. M. (1999). Religion and the judgement of obsessionality. Mental Health, Religion, and Culture, 2, 145-151.

Zea, M. C., Mason, M. A., \& Murguia, A. (2000). Psychotherapy with members of Latino/Latina religions and spiritual traditions. In P. S. Richards \& A. E. Bergin (Eds.), Handbook of psychotherapy and religious diversity (pp. 397-420). Washington, DC: American Psychological Association.

Zinnbauer, B. J., \& Pargament, K. I. (2005). Religiousness and spirituality. In R. F. Paloutzian \& C. L. Park (Eds.), Handbook of the psychology of religion and spirituality (pp. 21-42). New York: Guilford. 
$\underline{\text { Appendices }}$ 


\section{$\underline{\text { Appendix A }}$}

Participant Demographic Characteristics

Demographic Variable $n$ $\% *$ $M \quad S D \quad$ Range

Age

$54.4 \quad 9.1 \quad 30-84$

Sex:

Male

Female

Race/Ethnicity:

White/Caucasian

African/African American

Asian/Asian American

Hispanic/Latino(a)

Other

Highest Degree Obtained:

Ph.D.

Psy.D.

Ed.D.

Unspecified Doctorate
$63 \quad 44.7 \%$

$76 \quad 53.9 \%$
$129 \quad 91.5 \%$

$1 \quad 0.7 \%$

$3 \quad 2.1 \%$

$4 \quad 2.8 \%$

$2 \quad 1.4 \%$ 
$\underline{\text { Appendix A }}$, Continued

Participant Demographic Characteristics

Demographic Variable $n \quad \% * \quad M \quad S D \quad$ Range

Area of Specialization (Continued):

Neuropsychology

Other

Theoretical Orientation:

Cognitive Behavioral

Integrative

Eclectic

Psychoanalytic

Psychodynamic

Cognitive

Family Systems

Humanistic

Interpersonal

Other

Years Doing Testing

Years Doing Therapy

Hours Per Week Doing Therapy

* Total percentages do not equal $100 \%$ due to missing or multiple responses
$15.0 \quad 10.7 \quad 0-50$

$19.6 \quad 9.8 \quad 0-50$

$20.8 \quad 13.3 \quad 0-55$ 
$\underline{\text { Appendix A }}$, Continued

Participant Demographic Characteristics

Demographic Variable $n \quad \% * \quad M \quad S D \quad$ Range

Work Settings:

$\begin{array}{lll}\text { College/University } & 21 & 14.9 \% \\ \text { Hospital } & 20 & 14.2 \% \\ \text { Elementary/Secondary School } & 9 & 6.4 \% \\ \text { Public Clinic } & 1 & 0.7 \% \\ \text { Community Mental Health Center } & 11 & 7.8 \% \\ \text { Religious Setting } & 5 & 3.5 \% \\ \text { Private Practice } & 106 & 75.2 \% \\ \text { Prison/Corrections } & 4 & 2.8 \% \\ \text { Department of Veterans Affairs } & 2 & 1.4 \% \\ \text { Employee Assistance Program } & 2 & 1.4 \% \\ \text { Retired/Sabbatical } & 3 & 2.1 \% \\ \text { Rehabilitation } & 3 & 2.1 \% \\ \text { State/Govt. Agency/Program } & 3 & 2.1 \% \\ \text { Other } & 7 & 5.0 \%\end{array}$

Participating in Organized Religion:

$\begin{array}{lll}\text { Yes } & 64 & 45.4 \% \\ \text { No } & 75 & 53.2 \%\end{array}$

Times Per Month

$\begin{array}{lll}4.5 & 6.3 & 1-30\end{array}$

* Total percentages do not equal $100 \%$ due to missing or multiple responses 


\section{$\underline{\text { Appendix B }}$}

Participant Religious Demographics

\begin{tabular}{|c|c|c|c|c|}
\hline \multirow[t]{2}{*}{ Affiliation } & \multicolumn{2}{|c|}{ Religious Background } & \multicolumn{2}{|c|}{ Current Faith } \\
\hline & $n$ & $\% *$ & $n$ & $\% *$ \\
\hline Christian: & 114 & $80.9 \%$ & 57 & $40.4 \%$ \\
\hline Baptist & 6 & $4.3 \%$ & 2 & $1.4 \%$ \\
\hline Episcopal & 7 & $5.0 \%$ & 4 & $2.8 \%$ \\
\hline Latter Day Saint (Mormon) & 3 & $2.1 \%$ & 3 & $2.1 \%$ \\
\hline Lutheran & 9 & $6.4 \%$ & 7 & $5.0 \%$ \\
\hline Methodist & 23 & $16.3 \%$ & 5 & $3.5 \%$ \\
\hline Nondenominational & 4 & $2.8 \%$ & 5 & $3.5 \%$ \\
\hline Presbyterian & 6 & $4.3 \%$ & 1 & $0.7 \%$ \\
\hline Roman Catholic & 45 & $31.9 \%$ & 25 & $17.7 \%$ \\
\hline Other Christian & 11 & $7.8 \%$ & 5 & $3.5 \%$ \\
\hline Non-Christian: & 31 & $22.0 \%$ & 65 & $46.1 \%$ \\
\hline Baha'i & 1 & $0.7 \%$ & 2 & $1.4 \%$ \\
\hline Buddhist & 1 & $0.7 \%$ & 17 & $12.1 \%$ \\
\hline Hindu & 0 & $0.0 \%$ & 2 & $1.4 \%$ \\
\hline Jewish & 26 & $18.4 \%$ & 20 & $14.2 \%$ \\
\hline Muslim & 2 & $1.4 \%$ & 0 & $0.0 \%$ \\
\hline Taoist & 0 & $0.0 \%$ & 4 & $2.8 \%$ \\
\hline Unitarian Universalist & 1 & $0.7 \%$ & 7 & $5.0 \%$ \\
\hline
\end{tabular}

* Total percentages do not equal $100 \%$ due to multiple responses 
Appendix B, Continued

Participant Religious Demographics

\begin{tabular}{|c|c|c|c|c|}
\hline \multirow[t]{2}{*}{ Affiliation } & \multicolumn{2}{|c|}{ Religious Background } & \multicolumn{2}{|c|}{ Current Faith } \\
\hline & $n$ & $\% *$ & $n$ & $\% *$ \\
\hline Non-Christian (Continued): & 31 & $22.0 \%$ & 65 & $46.1 \%$ \\
\hline Other Religion & 0 & $0.0 \%$ & 13 & $9.2 \%$ \\
\hline Agnostic & 3 & $2.1 \%$ & 13 & $9.2 \%$ \\
\hline Atheist & 1 & $0.7 \%$ & 8 & $5.7 \%$ \\
\hline Nonreligious & 7 & $5.0 \%$ & 34 & $24.1 \%$ \\
\hline
\end{tabular}

* Total percentages do not equal 100\% due to multiple responses 


\section{$\underline{\text { Appendix C: }}$}

\section{Effect Sizes in Prior Research}

To determine an appropriate anticipated effect size to test the planned hypotheses, nine prior research studies were reviewed because of the relevance of their findings to the current study: Feeser (1997), Gerson, Allen, Gold, and Kose (2000), Gushue, Constantine, and Sciarra (2008), Hecker, Trepper, Wetchler, and Fontaine (1995), Hillowe (1985), Marcinkowski (1993), Minyard (1983), O'Connor and Vandenberg (2005), and Sanderson, Vandenberg, and Paese (1999). These studies investigated the influence of religious bias or multicultural competence on clinical judgments of pathology - the foundation of clinical judgment faith bias. All effect sizes reviewed for the current study were converted to $r$ for direct comparison to one another. For studies presenting multiple effect sizes, all relevant statistics were reduced to a single representative mean effect size for each main or interaction effect reported in order to avoid the bias which might result if a single study was overrepresented.

For the main effect of client/vignette religiousness on clinical judgments of pathology, this procedure yielded six effect sizes, one from each study reviewed: $r=.22$ (Feeser, 1997), $r=.29$ (Hillowe, 1985), $r=.20$ (Marcinkowski, 1993), $r=.40$ (Minyard, 1983), $r=.37$ (O’Connor \& Vandenberg, 2005), and $r=.47$ (Sanderson et al., 1999) (see Table 9). The overall mean of these six effect sizes is $r=.33$, a medium effect size (Cohen, 1988).

The attribute-by-treatment hypothesis of greatest interest to the current study, however, is the Resilience hypothesis. For the interaction effect of client/vignette 
religiousness and clinician religiousness on clinical judgments of pathology, three mean effect sizes were calculated: $r=.26$ (Feeser, 1997), $r=.39$ (Gerson et al., 2000), and $r=.13$ (Hillowe, 1985) (see Table 10). The overall mean of these three effect sizes is $r=.26$, a medium effect size (Cohen, 1988). The current study is a replication of Feeser's (1997) dissertation, in which a stepwise multiple regression analysis was used in an attribute-by-treatment design to investigate the contribution of clinician and client religious and spiritual orientation (and perceived client attractiveness) to overall prognosis. Based on this prior research, the predicted effect size for the current study was set at a medium effect size of $r=.26$ (Cohen, 1988). 
Table 9

Main Effect of Client/Vignette Religiousness on Clinical Judgments of Pathology From Previous Research

\begin{tabular}{|c|c|c|c|c|c|}
\hline Citation & $\begin{array}{l}\text { Original } \\
\text { ES }\end{array}$ & $N$ & Description & $\begin{array}{c}\text { Calculated } \\
\text { ES }\end{array}$ & $\begin{array}{l}\text { Mean } \\
|r|\end{array}$ \\
\hline Feeser (1997) & $T=2.24$ & 136 & $\begin{array}{l}\text { Contribution to overall } \\
\text { prognosis of Religious } \\
\text { Vignette }\end{array}$ & $r=.22$ & .22 \\
\hline $\begin{array}{l}\text { Hillowe } \\
\text { (1985) }\end{array}$ & $\begin{array}{l}F(1,113) \\
\quad=\end{array}$ & 115 & $\begin{array}{l}\text { Therapists rated religious } \\
\text { patients as: }\end{array}$ & & .29 \\
\hline & 34.31 & & Being more severely ill & $R^{2}=.13$ & \\
\hline & 26.31 & & Requiring more sessions & $R^{2}=.11$ & \\
\hline & 3.53 & & $\begin{array}{l}\text { Being less appropriate for } \\
\text { therapy }\end{array}$ & $R^{2}=.02$ & \\
\hline
\end{tabular}

Marcinkowski $\omega^{2}=.04 \quad 132$ Theistic vignettes received $\quad r=.20$ lower GAF scores

$r=\sqrt{\frac{t^{2}}{t^{2}+d f}}=\frac{d}{\sqrt{d^{2}+4}}=\sqrt{R^{2}}($ Becker, 2000; Cohen, 1988)

Cohen's $d=\sqrt{F\left(\frac{n_{t}+n_{c}}{n_{t} n_{c}}\right)\left(\frac{n_{t}+n_{c}}{n_{t}+n_{c}-2}\right)}$ (Thalheimer \& Cook, 2002)

$R^{2}=\frac{\omega^{2} /\left(1-\omega^{2}\right)}{1+\left[\omega^{2} /\left(1-\omega^{2}\right)\right]}$ based on $f=\sqrt{\frac{\omega^{2}}{1-\omega^{2}}}$ and $f^{2}=\frac{R^{2}}{1-R^{2}} ; r=\sqrt{R^{2}}$ (Cohen, 1988) 
Table 9, Continued

Main Effect of Client/Vignette Religiousness on Clinical Judgments of Pathology From Previous Research

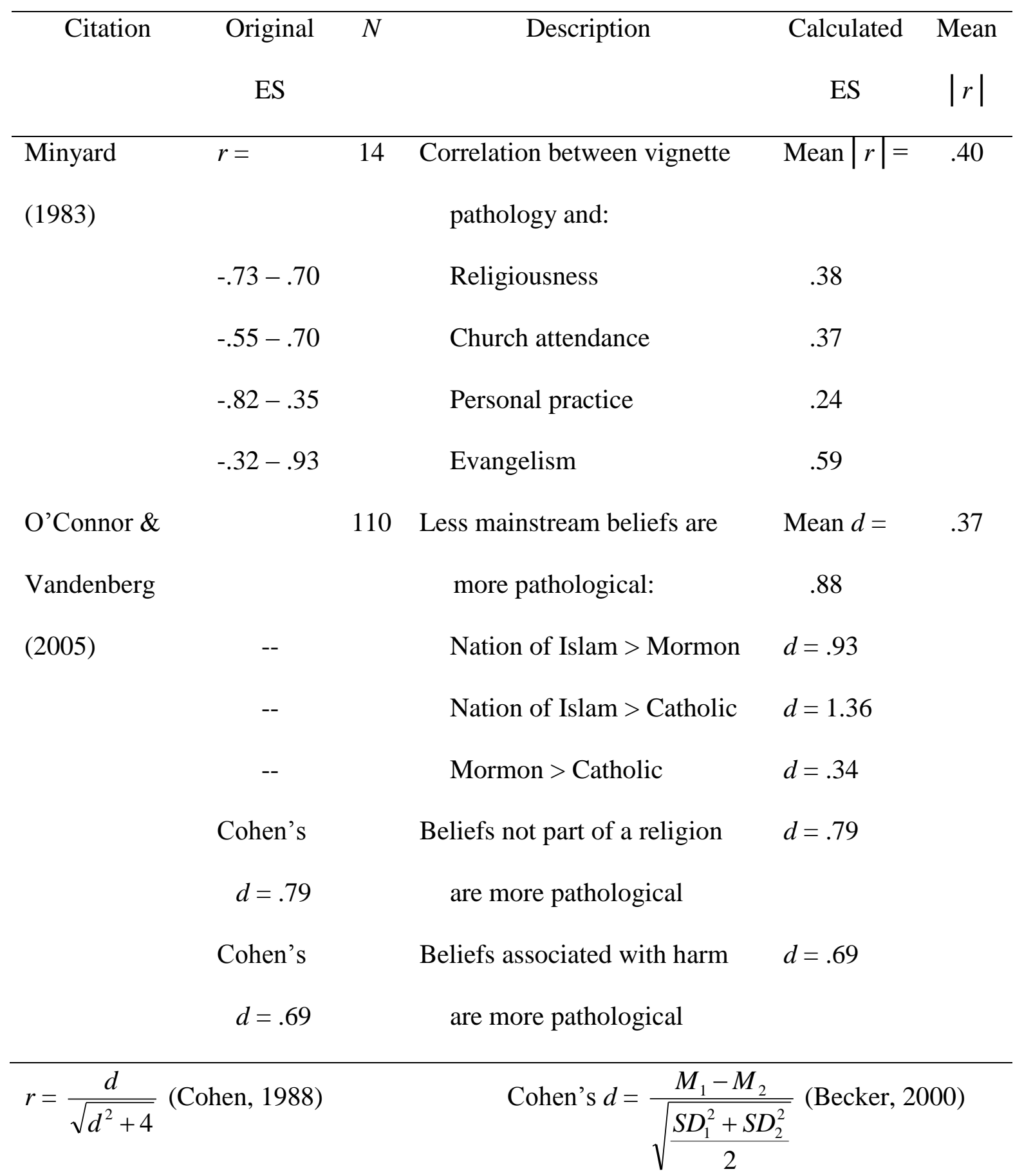


Table 9, Continued

Main Effect of Client/Vignette Religiousness on Clinical Judgments of Pathology From Previous Research

\begin{tabular}{lccccc}
\hline \multicolumn{1}{c}{ Citation } & Original & $N$ & Description & Calculated & Mean \\
& ES & & & ES & $|r|$ \\
\hline Sanderson, & $r=.47$ & 67 & Overall correlation between & $r=.47$ & .47 \\
Vandenberg, \& & & & clinician ratings of \\
Paese (1999) & \multicolumn{3}{c}{ religious authenticity and } \\
& \multicolumn{3}{c}{ mental health } \\
\end{tabular}


Table 10

Interaction Effect of Client/Vignette Religiousness and Clinician Religiousness on Clinical Judgments of Pathology From Previous Research

\begin{tabular}{|c|c|c|c|c|c|}
\hline \multirow[t]{2}{*}{ Citation } & Original & $N$ & Description & Calculated & Mean \\
\hline & $\mathrm{ES}$ & & & ES & $|r|$ \\
\hline \multirow[t]{6}{*}{ Feeser (1997) } & & 136 & Contribution to overall prognosis of: & & .26 \\
\hline & $t=2.57$ & & Religious Vignette + & $r=.25$ & \\
\hline & $t=2.49$ & & Clinician Extrinsic Orientation & $r=.24$ & \\
\hline & $t=3.44$ & & Religious Vignette + & $r=.33$ & \\
\hline & $t=2.60$ & & Clinician Extrinsic Orientation + & $r=.25$ & \\
\hline & $t=2.35$ & & Spiritual Vignette & $r=.23$ & \\
\hline Gerson, Allen, & $r=-.39$ & 87 & Main effect correlation between & $r=-.39$ & .39 \\
\hline \multicolumn{2}{|l|}{ Gold, \& Kose } & & clinician religiousness and clinical & & \\
\hline \multicolumn{2}{|l|}{$(2000)$} & & ratings of a religious client & & \\
\hline \multirow[t]{6}{*}{ Hillowe (1985) } & $R^{2}=$ & 115 & Inverse correlation between therapist & $R^{2}=.01$ & .13 \\
\hline & $.0015-$ & & religious beliefs and overall & & \\
\hline & .0402 & & prognosis for religious patients & & \\
\hline & $R^{2}=$ & & Direct correlation between therapist & $R^{2}=.02$ & \\
\hline & .00004 & & nondoctrinal beliefs and overall & & \\
\hline & -.0477 & & prognosis for religious patients & & \\
\hline \multicolumn{2}{|l|}{ Mean Effect Size } & & & & .26 \\
\hline$\sqrt{\frac{t^{2}}{t^{2}+d f}}=$ & $\overline{\mathrm{R}^{2}}(\mathrm{~B}$ & & $0 ;$ Cohen, 1988) & & \\
\hline
\end{tabular}


Appendix D:

\title{
Clinical Judgment: The Impact of Client and Clinician Variables
}

\author{
Brief Survey Booklet
}




\title{
Appendix D1:
}

\section{Case Vignette}

\begin{abstract}
Please read the following description of Mary, a fictional client, and answer the questions that follow. Please answer the questions as they appear and do not read ahead.
\end{abstract}

Mary is a 20-year-old White/Caucasian female who is an undergraduate student. She was apprehensive during the initial interview and seemed embarrassed about her problems. Her presenting complaint is that she has many worries. She is having difficulty reading, studying, and concentrating because she has become increasingly preoccupied with her worries over the past year. She spends hours each night rehashing the day's events, especially interactions with her professors and exchanges with other students in a particularly demanding course she is taking, but also some interactions with her parents, with whom she finds it difficult to get along. She says that she endlessly tries to correct in her mind any mistakes she might have made. She runs over every event, asking herself if she behaved properly or said the right thing. She does this at her desk when she is supposed to be reading, doing homework, or working on her undergraduate thesis, which interferes with her concentration. It is not unusual for her to look at the clock after such a period of rumination and find, to her surprise, that two or three hours have elapsed. She also does this at night, so she has not been sleeping well lately and finds herself unusually irritable and easily fatigued. She worries about a number of everyday, real-life problems and feels unable to stop her mind from worrying. For the past year, Mary has had fewer social contacts because of her nervous symptoms. Although she sometimes misses classes because of her symptoms, she continues to keep her grades at a B average. She tends to hide her symptoms from her family and her roommate and reports few problems with them. Recent medical and neurological examinations have ruled out substance use, general medical conditions, and neurological impairment as factors contributing to her problems. 
Mary considers herself to be a born-again Christian and describes herself as "truly devoted to Jesus." She views Jesus as the source of her strength and happiness, both directly and indirectly. The church services she attends typically last 3 or 4 hours and involve singing, giving testimony, and praising Jesus. Privately, she prays and reads the Bible several times a day, decorates her home with religious items, and talks to others about what she believes. She volunteers two or three times a month at a local homeless shelter and often gives what little money she has to her church to support its overseas missions and foreign ministries, seeing her community involvement as "my privilege as a true Christian woman." She feels strongly that "it's not enough for me just to sit in the pew - I need to be involved in meeting the needs others have of clothing, shelter, food, and friendship in order to truly serve the Lord."

[Paragraph 2 has six different versions - See Appendix H] 


\section{Appendix D2:}

\section{The Schedule of Therapists' Prognostic Expectations (STPE)}

\section{Scale 1}

1.) How appropriate a candidate for psychotherapy is this client?

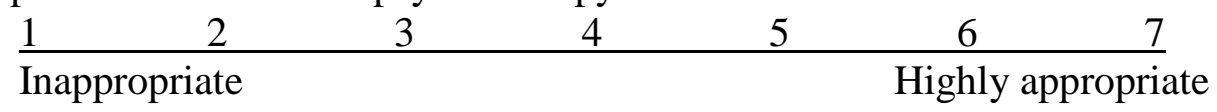

2.) Would you choose to work with this client?

\begin{tabular}{cccccc}
1 & 3 & 4 & 5 & 6 & 7 \\
\hline Definitely would not & Unsure & & Definitely would
\end{tabular}

3.) What is the degree of impairment of the client's psychological functioning?

\begin{tabular}{llllllll}
1 & 2 & 3 & 4 & 5 & 6 & 7 \\
\hline Severe & & & Moderate & & & Mild
\end{tabular}

4.) How motivated is the client to change?

\begin{tabular}{cccccc}
1 & 3 & 4 & 5 & 6 & 7 \\
\hline Not at all motivated & & & & Highly motivated
\end{tabular}

5.) What is the client's capacity for psychological insight?

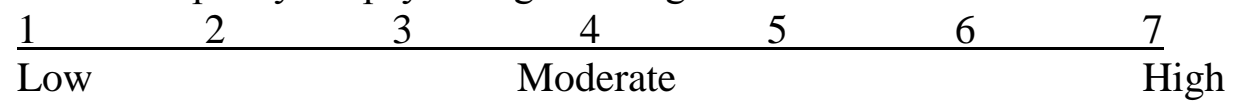

6.) How likely is it that the client will make substantial progress in psychotherapy?

\begin{tabular}{ccccccc}
1 & 2 & 3 & 4 & 5 & 6 & 7 \\
\hline Highly unlikely & & & & & Highly likely
\end{tabular}

7.) What DSM-IV diagnosis would you give the client?

Axis I:

Axis $\mathrm{I}$ :

Axis II:

Axis V: GAF (Current):

8.) What is your estimate of the number of therapy sessions required for the client to make substantial progress?

9.) How many therapy sessions do you think the client would actually attend? 


\section{Global Assessment of Functioning (GAF) Scale}

\section{Consider psychological, social, and occupational functioning on a hypothetical continuum of mental health-illness. Do not include impairment in functioning due to physical (or environmental) limitations.}

100 - Superior functioning in a wide range of activities, life's problems never seem to get out of hand, is sought out by others because of his or her many positive qualities. No

91 - symptoms.

90 - Absent or minimal symptoms (e.g., mild anxiety before an exam), good functioning in all areas, interested and involved in a wide range of activities, socially effective, generally satisfied with life, no more than everyday problems or concerns (e.g., an

81 - occasional argument with family members).

80 - If symptoms are present, they are transient and expectable reactions to psychosocial stressors (e.g., difficulty concentrating after family argument); no more than slight 71 - impairment in social, occupational, or school functioning (e.g., temporarily falling

70 - Some mild symptoms (e.g., depressed mood and mild insomnia) or some difficulty in social, occupational, or school functioning (e.g. occasional truancy, or theft within the

61 - household), but generally functioning pretty well, has some meaningful interpersonal relationships.

60 - Moderate symptoms (e.g., flat affect and circumstantial speech, occasional panic attacks) or moderate difficulty in social, occupational, or school functioning (e.g., few

$\mathbf{5 1}$ - friends, conflicts with peers or co-workers).

50 - Serious symptoms (e.g. suicidal ideation, severe obsessional rituals, frequent shoplifting) or any serious impairment in social, occupational, or school functioning

41 -(e.g., no friends, unable to keep a job).

40 - Some impairment in reality testing or communication (e.g., speech is at times illogical, obscure, or irrelevant) or major impairment in several areas, such as work or school, family relations, judgment, thinking, or mood (e.g., depressed man avoids friends, neglects family, and is unable to work; child frequently beats up younger

31 - children, is defiant at home, and is failing at school).

30 - Behavior is considerably influenced by delusions or hallucinations or serious impairment in communication or judgment (e.g., sometimes incoherent, acts grossly inappropriately, suicidal preoccupation) or inability to function in almost all areas

21 -(e.g., stays in bed all day; no job, home, or friends).

20 - Some danger of hurting self or others (e.g., suicide attempts without clear expectation of death; frequently violent; manic excitement) or occasionally fails to maintain minimum personal hygiene (e.g., smears feces) or gross impairment in

11 - communication (e.g., largely incoherent or mute).

10 - Persistent danger of severely hurting self or others (e.g. recurrent violence) or persistent inability to maintain minimum personal hygiene or serious suicidal act 1 - with clear expectation of death.

$\mathbf{0}$ - Inadequate information 
How likely is it that this client has the following diagnoses?

Not Very Likely $\quad$ Very Likely

10.) Panic Disorder (with or without Agoraphobia)

\begin{tabular}{lllllll}
1 & 2 & 3 & 4 & 5 & 6 & 7 \\
\hline
\end{tabular}

11.) Agoraphobia (without history of Panic Disorder)

12.) Obsessive-Compulsive Disorder

\begin{tabular}{lllllll}
1 & 2 & 3 & 4 & 5 & 6 & 7 \\
\hline
\end{tabular}

13.) Generalized Anxiety Disorder

\begin{tabular}{lllllll}
1 & 2 & 3 & 4 & 5 & 6 & 7 \\
\hline
\end{tabular}

14.) Posttraumatic Stress Disorder

\begin{tabular}{lllllll}
1 & 2 & 3 & 4 & 5 & 6 & 7 \\
\hline
\end{tabular}

15.) Social Phobia

$\begin{array}{lllllll}1 & 2 & 3 & 4 & 5 & 6 & 7\end{array}$

16.) Adjustment Disorder with Anxiety

\begin{tabular}{lllllll}
1 & 2 & 3 & 4 & 5 & 6 & 7 \\
\hline
\end{tabular}

17.) Acute Stress Disorder

\begin{tabular}{lllllll}
1 & 2 & 3 & 4 & 5 & 6 & 7 \\
\hline
\end{tabular}

18.) Any Other Anxiety Disorder not listed above (such as Specific Phobia, Anxiety Disorder Due to a General Medical Condition, Substance-Induced Anxiety Disorder, or Anxiety Disorder NOS)

If you rated this as 4-7, please indicate the diagnosis:

\section{Comments (optional):}




\section{Appendix D3:}

\section{The Multicultural Counseling Knowledge and Awareness Scale (MCKAS)}

\section{Scale 2}

Using the given scale, rate the truth of each item as it applies to you.

1.) I believe all clients should maintain direct eye contact during counseling.

2.) I check up on my minority/cultural counseling skills by monitoring my functioning - via consultation, supervision, and continuing education.

3.) I am aware some research indicates that minority clients receive "less preferred" forms of counseling treatment than majority clients.

4.) I think that clients who do not discuss intimate aspects of their lives are being resistant and defensive.

5.) I am aware of certain counseling skills, techniques, or approaches that are more likely to transcend culture and be effective with any clients.

6.) I am familiar with the "culturally deficient" and "culturally deprived" depictions of minority mental health and understand how these labels serve to foster and perpetuate discrimination.

7.) I feel all the recent attention directed toward multicultural issues in counseling is overdone and not really warranted.

8.) I am aware of individual differences that exist among members within a particular ethnic group based on values, beliefs, and level of acculturation.

\begin{tabular}{|c|c|c|}
\hline 2 & 5 & 6 \\
\hline Not at Al & Somewhat & Totally \\
\hline
\end{tabular}

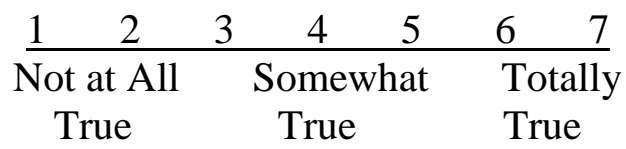

\begin{tabular}{|c|c|c|}
\hline 2 & 5 & 6 \\
\hline $\begin{array}{l}\text { Not at } \mathrm{Al} \\
\text { True }\end{array}$ & $\begin{array}{l}\text { Somewhat } \\
\text { True }\end{array}$ & $\begin{array}{l}\text { Totally } \\
\text { True }\end{array}$ \\
\hline
\end{tabular}

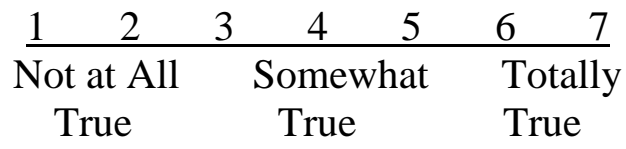

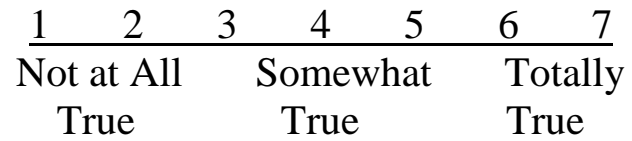

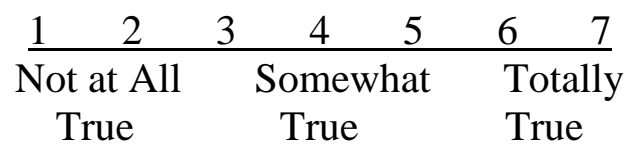

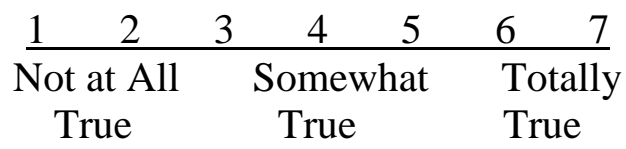

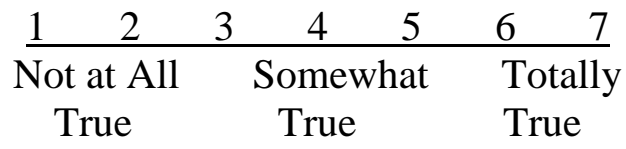


9.) I am aware some research indicates that minority clients are more likely to be diagnosed with mental illnesses than are majority clients.

10.) I think that clients should perceive the nuclear family as the ideal social unit.

11.) I think that being highly competitive and achievement oriented are traits that all clients should work towards.

12.) I am aware of the differential interpretations of nonverbal communication (e.g., personal space, eye contact, handshakes) within various racial/ethnic groups.

13.) I understand the impact and operations of oppression and the racist concepts that have permeated the mental health professions.

14.) I realize that counselor-client incongruities in problem conceptualization and counseling goals may reduce counselor credibility.

15.) I am aware that some racial/ethnic minorities see the profession of psychology functioning to maintain and promote the status and power of the White Establishment.

16.) I am knowledgeable of acculturation models for various ethnic minority groups.

17.) I have an understanding of the role culture and racism play in the development of identity and worldviews among minority groups.

18.) I believe that it is important to emphasize objective and rational thinking in minority clients.

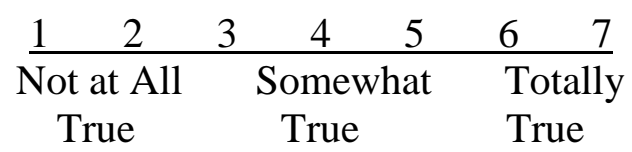

\begin{tabular}{|c|c|c|}
\hline 2 & 5 & 6 \\
\hline $\begin{array}{l}\text { Not at Al } \\
\text { True }\end{array}$ & $\begin{array}{l}\text { Somewhat } \\
\text { True }\end{array}$ & $\begin{array}{l}\text { Totally } \\
\text { True }\end{array}$ \\
\hline
\end{tabular}

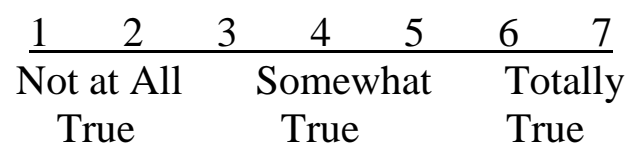

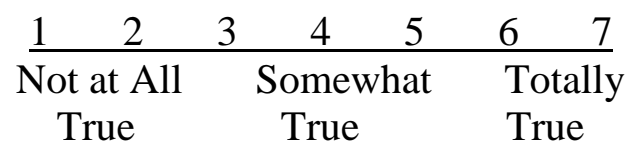

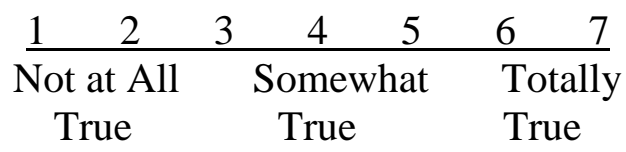

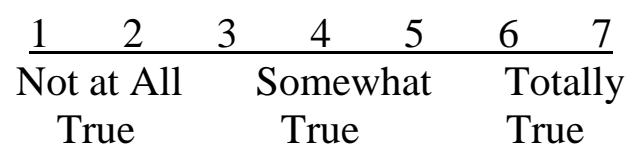

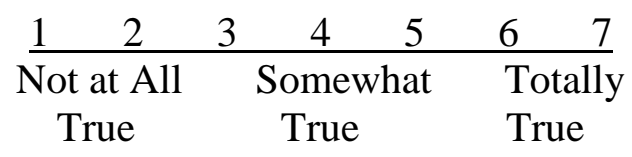

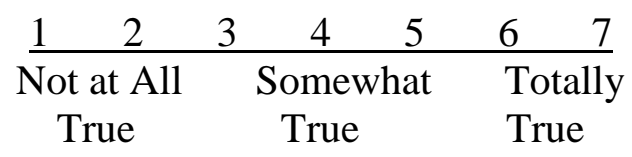

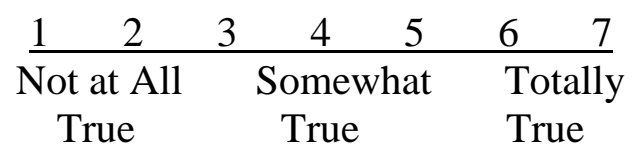

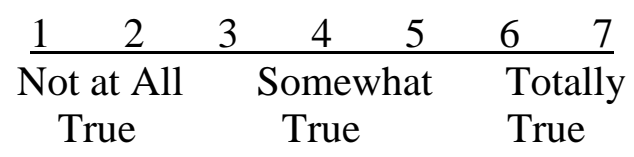


19.) I am aware of culture-specific (culturally indigenous) models of counseling for various racial/ethnic groups.

20.) I believe that my clients should view a patriarchal structure as the ideal.

21.) I am aware of both the initial barriers and benefits related to the cross-cultural counseling relationship.

22.) I am comfortable with differences that exist between me and my clients in terms of race and beliefs.

23.) I am aware of institutional barriers which may inhibit minorities from using mental health services.

24.) I think that my clients should exhibit some degree of psychological mindedness and sophistication.

25.) I believe that minority clients will benefit most from counseling with a majority who endorses White middle-class values and norms.

26.) I am aware that being born a White person in this society carries with it certain advantages.

27.) I am aware of the value assumptions inherent in major schools of counseling and understand how these assumptions may conflict with values of culturally diverse clients.

28.) I am aware that some minorities see the counseling process as contrary to their own life experiences and inappropriate or insufficient to their needs.

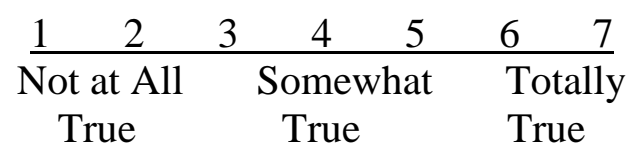

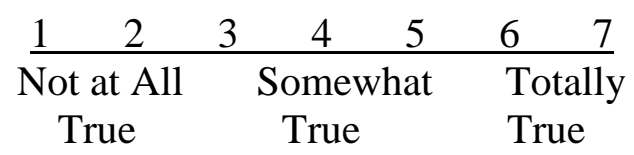

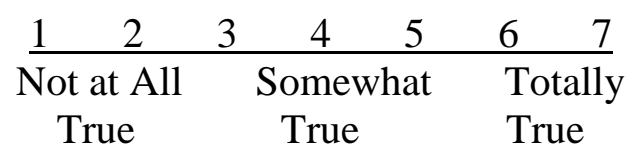

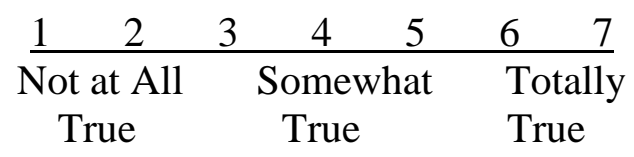

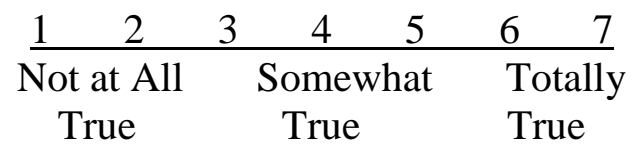

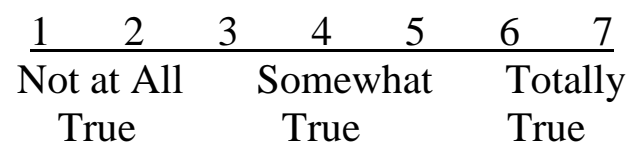

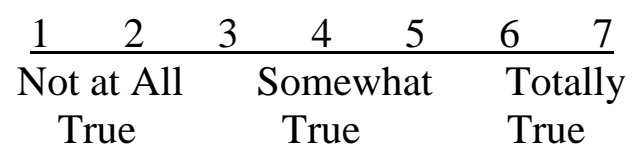

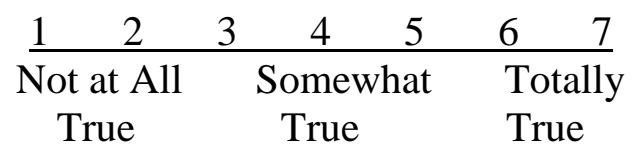

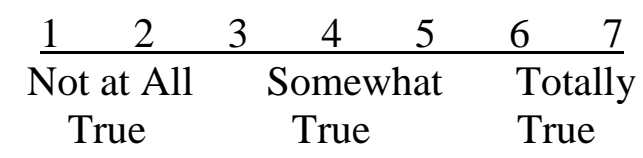

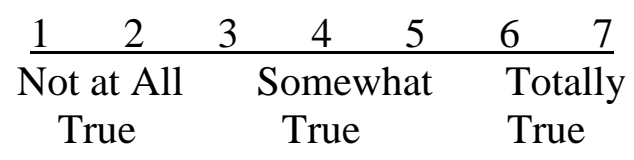


29.) I am aware that being born a minority in this society brings with it certain challenges that White people do not have to face.

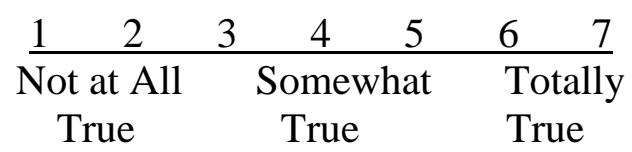

30.) I believe that all clients must view themselves as their number one responsibility.

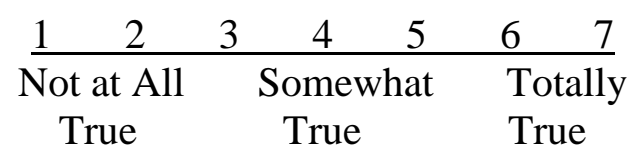

31.) I am sensitive to circumstances (personal biases, language dominance, stage of ethnic identity development) which may dictate referral of the minority client to a member of

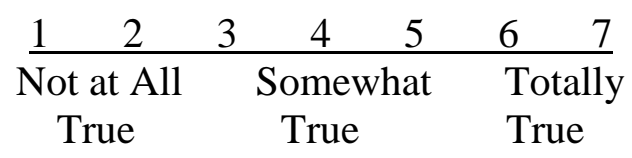
his/her own racial/ethnic group.

32.) I am aware that some minorities believe counselors lead minority students into nonacademic programs regardless of student potential, preferences, or ambitions.

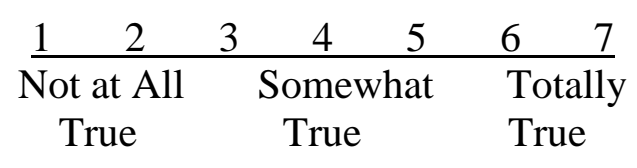

Thank you for completing this instrument. Please feel free to express in writing below any thoughts, concerns, or comments you have regarding this instrument. 


\section{Appendix D4:}

\section{The Spiritual - Religious Orientation Scale (S-ROS)}

Scale 3

Please rate your agreement with each of the following items.

Please be sure that all items have been marked-leave no items unanswered.

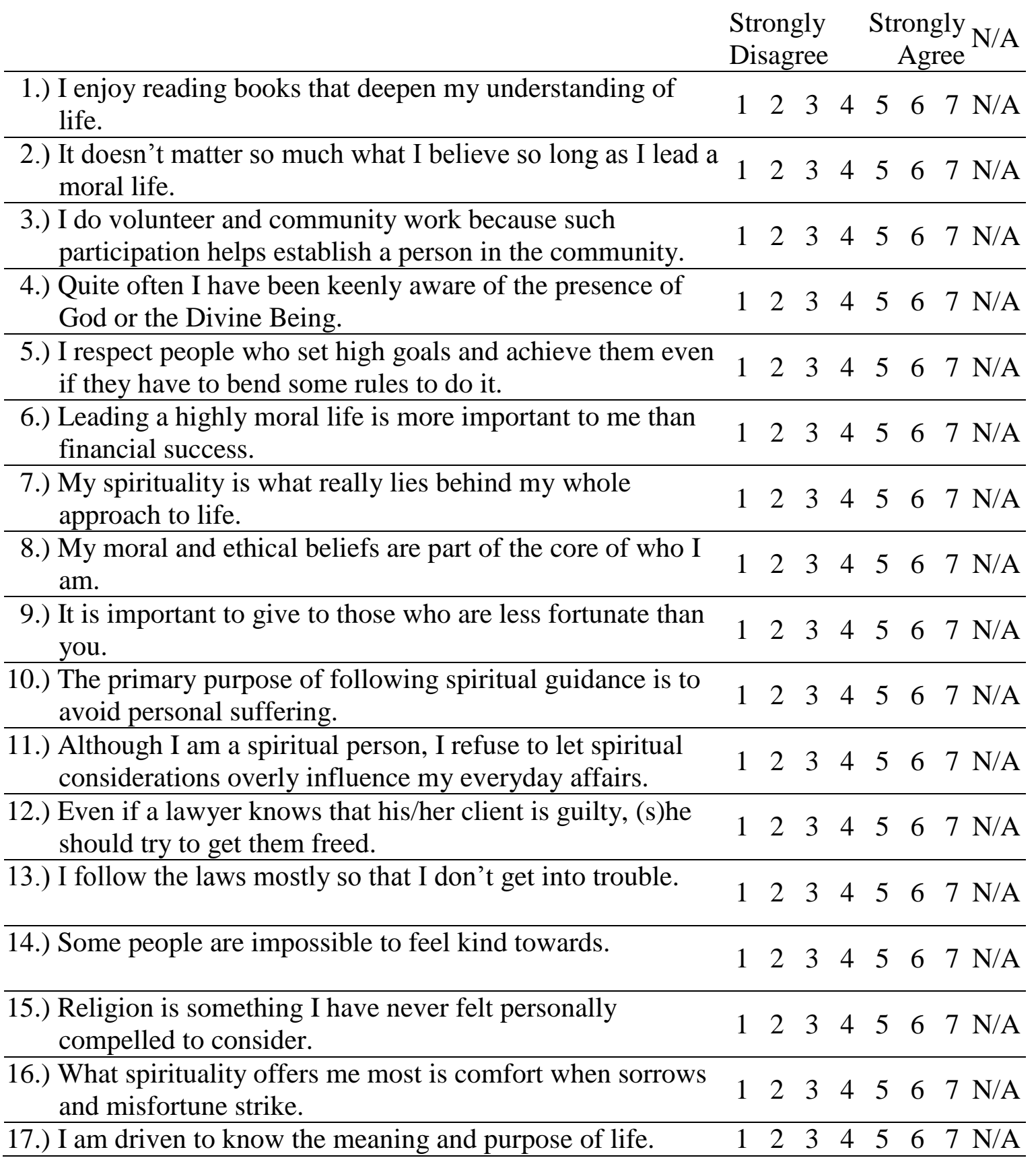


18.) If someone hurts you on purpose, it is $\mathrm{OK}$ to do something to even the score.

$\begin{array}{llllllll}1 & 2 & 3 & 4 & 5 & 6 & 7 & \text { N/A }\end{array}$

19.) I try hard to carry my spiritual beliefs over into all my other dealings in life.

20.) If the salesperson at a large department store gave me too much change, I would return it.

$\begin{array}{llllllll}1 & 2 & 3 & 4 & 5 & 6 & 7 & \text { N/A }\end{array}$

$\begin{array}{llllllll}1 & 2 & 3 & 4 & 5 & 6 & 7 & \text { N/A }\end{array}$

21.) My spiritual activities are important as a way to meet likeminded people.

$\begin{array}{llllllll}1 & 2 & 3 & 4 & 5 & 6 & 7 & \text { N/A }\end{array}$

22.) Unfortunately, it is sometimes necessary to deceive others.

$\begin{array}{llllllll}1 & 2 & 3 & 4 & 5 & 6 & 7 & \text { N/A }\end{array}$

23.) It is important for me to spend periods of time in private reflective thought and meditation.

$\begin{array}{llllllll}1 & 2 & 3 & 4 & 5 & 6 & 7 & \text { N/A }\end{array}$

24.) Living a comfortable, pleasurable life is very important to me.

$\begin{array}{llllllll}1 & 2 & 3 & 4 & 5 & 6 & 7 & \text { N/A }\end{array}$

25.) Life has no meaning except what we make of it.

$\begin{array}{llllllll}1 & 2 & 3 & 4 & 5 & 6 & 7 & \text { N/A }\end{array}$

26.) It doesn't matter if I belong to a (church, synagogue, temple) so long as I treat others fairly.

$\begin{array}{llllllll}1 & 2 & 3 & 4 & 5 & 6 & 7 & \text { N/A }\end{array}$

27.) I am as honest when I know I wouldn't get caught as when I might get caught.

$\begin{array}{llllllll}1 & 2 & 3 & 4 & 5 & 6 & 7 & \text { N/A }\end{array}$

28.) Planning to steal something is almost as bad as actually stealing it.

$\begin{array}{llllllll}1 & 2 & 3 & 4 & 5 & 6 & 7 & \text { N/A }\end{array}$

29.) The purpose of meditation is to promote a happy and peaceful life.

$\begin{array}{llllllll}1 & 2 & 3 & 4 & 5 & 6 & 7 & \text { N/A }\end{array}$

30.) If I could get into a movie without paying and be sure I was not seen, I would probably do it.

$\begin{array}{llllllll}1 & 2 & 3 & 4 & 5 & 6 & 7 & \text { N/A }\end{array}$

31.) Although I consider myself a spiritual person, I feel there are many more important things in life.

$\begin{array}{llllllll}1 & 2 & 3 & 4 & 5 & 6 & 7 & \text { N/A }\end{array}$

32.) On occasion, I take advantage of people.

$\begin{array}{llllllll}1 & 2 & 3 & 4 & 5 & 6 & 7 & \text { N/A }\end{array}$

33.) Being successful at what I do makes my life meaningful.

34.) I would never think of letting someone else be punished for my wrongdoing.

$\begin{array}{llllllll}1 & 2 & 3 & 4 & 5 & 6 & 7 & \text { N/A }\end{array}$

35.) Helping a friend in need is a duty I take seriously.

$\begin{array}{llllllll}1 & 2 & 3 & 4 & 5 & 6 & 7 & \text { N/A }\end{array}$

$\begin{array}{llllllll}1 & 2 & 3 & 4 & 5 & 6 & 7 & \text { N/A }\end{array}$

36.) Spirituality is especially important to me because it answers many questions about the meaning of life.

$\begin{array}{llllllll}1 & 2 & 3 & 4 & 5 & 6 & 7 & \text { N/A }\end{array}$

37.) I sometimes try to get even, rather than to forgive and forget.

$\begin{array}{llllllll}1 & 2 & 3 & 4 & 5 & 6 & 7 & \text { N/A }\end{array}$

38.) What my philosophy of life offers me most is comfort when sorrows and misfortune strike.

$\begin{array}{llllllll}1 & 2 & 3 & 4 & 5 & 6 & 7 & \text { N/A }\end{array}$

39.) The purpose of following ethical standards is to promote a happy and peaceful life. 
40.) I treat others fairly so that they will like me.

$\begin{array}{llllllll}1 & 2 & 3 & 4 & 5 & 6 & 7 & \text { N/A }\end{array}$

41.) I believe the universe was divinely created.

$\begin{array}{llllllll}1 & 2 & 3 & 4 & 5 & 6 & 7 & \text { N/A }\end{array}$

42.) All major life events have a deeper meaning and purpose.

$\begin{array}{llllllll}1 & 2 & 3 & 4 & 5 & 6 & 7 & \text { N/A }\end{array}$

43.) What religion offers me most is comfort when sorrows and misfortune strike.

$\begin{array}{llllllll}1 & 2 & 3 & 4 & 5 & 6 & 7 & \text { N/A }\end{array}$

44.) The purpose of prayer is to secure a happy and peaceful life.

$\begin{array}{llllllll}1 & 2 & 3 & 4 & 5 & 6 & 7 & \text { N/A }\end{array}$

45.) The primary purpose of prayer is to gain relief and protection.

$\begin{array}{llllllll}1 & 2 & 3 & 4 & 5 & 6 & 7 & \text { N/A }\end{array}$

46.) One reason for my being a congregation member is that such membership helps to establish a person in the $\quad \begin{array}{llllllll}1 & 2 & 3 & 4 & 5 & 6 & 7 & \text { N/A }\end{array}$ community.

47.) My house of worship is most important as a place to formulate good social relations.

$\begin{array}{llllllll}1 & 2 & 3 & 4 & 5 & 6 & 7 & \text { N/A }\end{array}$

48.) A primary reason for my interest in religion is that my house of worship is a congenial social activity.

$\begin{array}{llllllll}1 & 2 & 3 & 4 & 5 & 6 & 7 & \text { N/A }\end{array}$

49.) I try hard to carry my religion over into all my other dealings in life.

$\begin{array}{llllllll}1 & 2 & 3 & 4 & 5 & 6 & 7 & \text { N/A }\end{array}$

50.) My religious beliefs are what really lie behind my whole approach to life.

$\begin{array}{llllllll}1 & 2 & 3 & 4 & 5 & 6 & 7 & \text { N/A }\end{array}$

51.) Religion is especially important to me because it answers many questions about the meaning of life.

$\begin{array}{llllllll}1 & 2 & 3 & 4 & 5 & 6 & 7 & \text { N/A }\end{array}$

52.) I read literature about my faith.

$\begin{array}{llllllll}1 & 2 & 3 & 4 & 5 & 6 & 7 & \text { N/A }\end{array}$

53.) It is important to me to spend periods of time in private religious thought and meditation.

$\begin{array}{llllllll}1 & 2 & 3 & 4 & 5 & 6 & 7 & \text { N/A }\end{array}$

54.) Although I am a religious person, I refuse to let religious considerations influence my everyday affairs.

$\begin{array}{llllllll}1 & 2 & 3 & 4 & 5 & 6 & 7 & \text { N/A }\end{array}$

55.) Although I believe in my religion, I feel there are many more important things in life. 


\section{Appendix D5:}

\section{The Religious Issues in Therapy Survey Form (RITSF)}

\section{Scale 4}

Please indicate your agreement or disagreement with each of the following cases.

1.) Religious belief tends to detract from one's intellectual development.

2.) Religious belief tends to encourage selfresponsibility.

3.) Religious belief tends to be a way of avoiding reality.

4.) Religious belief often prevents people from making effective attempts at changing themselves or their environment.

5.) Religious belief provides a moral base for those who would rather not establish moral guidelines themselves.

6.) Religious belief is a neurosis.

7.) Religious belief helps control expressions of personal disorders.

8.) Religious belief is too subjective to be useful in therapy.

9.) Religious belief aids in integrating people with their society.

10.) Religious activities effectively change one's perceptions of circumstances.

11.) Religious belief promotes a sense of helplessness.

12.) Religious belief tends to foster high self-esteem.

13.) Religious belief is very relevant to the therapy process.

14.) Helping clients strengthen spiritual expressions is a legitimate goal for therapy.

15.) Therapists who introduce religious issues into therapy are acting unethically.

16.) Counseling clients on religious issues is within my therapeutic competence.

17.) Referral to clergy is usually the best way to handle any religious issue.

18.) Few clients wish to discuss their religious beliefs.

19.) I consider myself deeply religious.
Strongly Disagree Unsure Agree Strongly

Disagree

SD $\quad \mathrm{D} \quad \mathrm{U}$ A $\mathrm{SA}$

SD $\quad$ D $\quad$ U $\quad$ A $\quad$ SA

SD $\quad$ D $\quad$ U $\quad$ A $\quad$ SA

SD $\quad \mathrm{D} \quad \mathrm{U} \quad \mathrm{A} \quad \mathrm{SA}$

SD $\quad$ D $\quad$ U A $\quad$ SA

SD $\quad$ D $\quad$ U $\quad$ A $\quad$ SA

SD $\quad$ D $\quad$ U $\quad$ A $\quad$ SA

SD $\quad$ D $\quad$ U A $\quad$ SA

SD $\quad$ D $\quad$ U $\quad$ A $\quad$ SA

SD $\quad$ D $\quad$ U A $\quad$ SA

SD $\quad$ D $\quad$ U $\quad$ A $\quad$ SA

SD $\quad$ D $\quad$ U $\quad$ A $\quad$ SA

SD $\quad$ D $\quad$ U $\quad$ A $\quad$ SA

SD $\quad$ D $\quad$ U $\quad$ A $\quad$ SA

SD $\quad$ D $\quad$ U $\quad$ A $\quad$ SA

SD $\quad$ D $\quad$ U A $\quad$ SA

SD $\quad$ D $\quad$ U $\quad$ A $\quad$ SA

SD $\quad \mathrm{D} \quad \mathrm{U} \quad \mathrm{A}$ SA

SD $D \quad$ U A $\quad$ SA




\section{Appendix D6:}

\section{Demographic Questions}

1.) Age: years old

2.) Sex: (check one)

Male

Female

3.) Race/Ethnicity: (check all that apply) Caucasian/ White Asian Native American

* Please Specify:

African American/ Black Hispanic/ Latino(a) Other*

4.) Highest degree obtained: (check one, then specify type - e.g., MSW, Clinical Psychology Psy.D., Counseling Psychology Ph.D., etc.)

Bachelor's Degree: Masters Degree:

Doctorate:

Other:

5.) What is your main theoretical orientation? (e.g., Integrative, Psychoanalytic, CBT)

6.) Since obtaining your degree, how many years have you been doing:

Diagnostic testing? years

Psychotherapy? years

7.) How many hours per week do you spend doing psychotherapy (i.e., individual, family, couples, or group therapy)? hours/week

8.) What are the settings in which you work? (Check all that apply. If you work in more than one setting, please rank the settings in order of time in which you spend in each, i.e., $1=$ primary setting, $2=$ secondary setting, etc.)

College/University

Elementary/Secondary school Community Mental Health Center Private Practice
Hospital

Public Clinic Religious Setting Other (specify) 
9.) What was your predominant religious background as you were growing up? (Check one.)

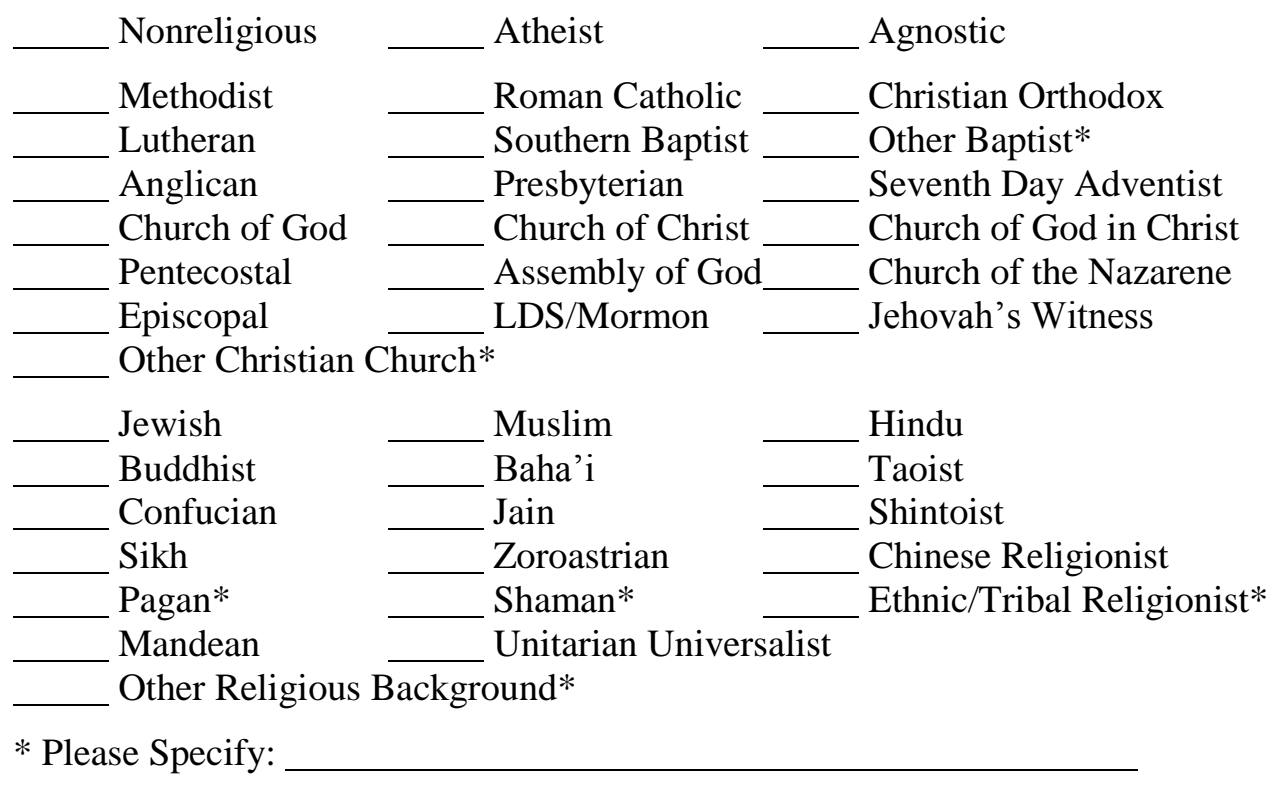

10.) Describe yourself today. (check as many as apply)

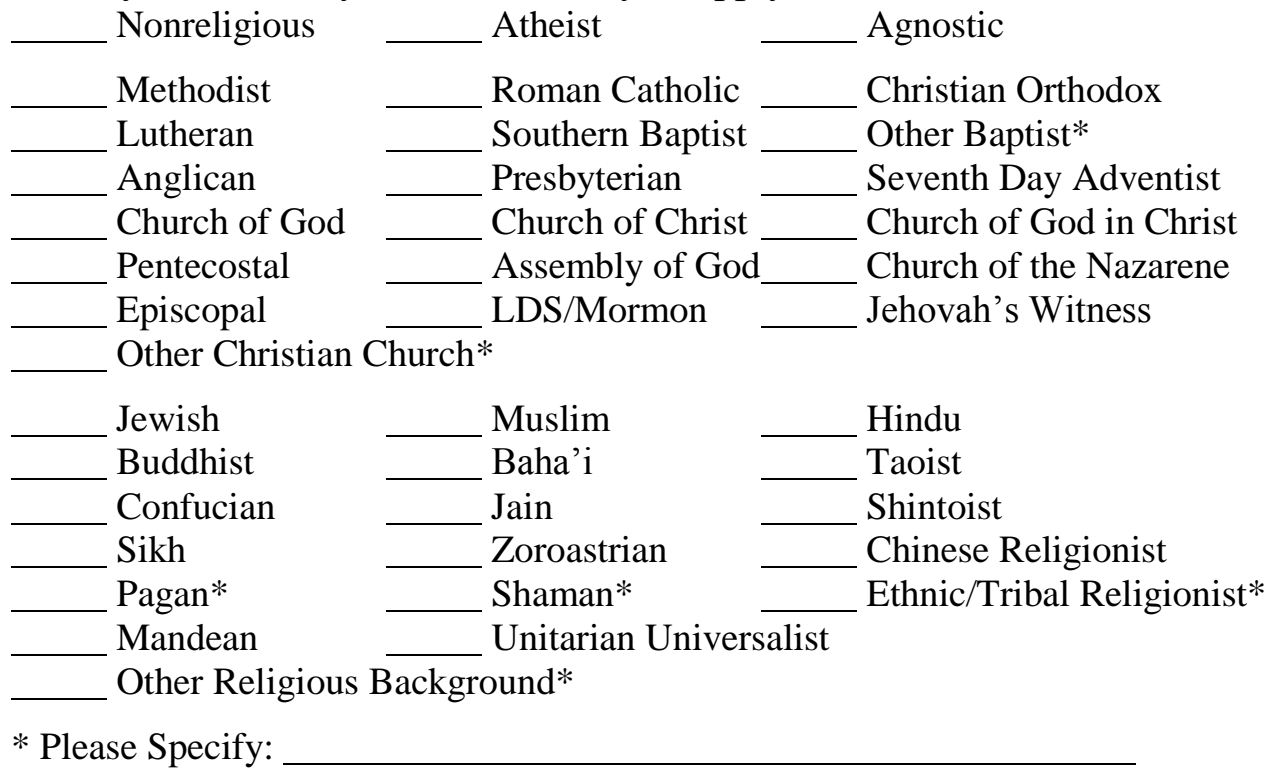

11.) Are you participating in any organized religious body now?

No Yes Where?

How often? 


\section{Appendix D7:}

\section{Debriefing Information}

\section{Clinical Judgment: The Impact of Client and Clinician Variables}

For debriefing information, please visit the following website:

\section{http://kaharris.iweb.bsu.edu/cjfb.htm}

This website is also printed on the introductory letter, which does not need to be returned with the rest of the survey materials and which you may keep. Please do not visit this website until after you have completed and returned the survey packet, as visiting this site will give away exactly what this study is investigating and may bias your responses if you have not already completed the survey. Thank you for your cooperation.

I sincerely appreciate your time and effort.

Thanks so much for completing this survey!

Comments: (optional) 
Appendix E:

Survey Correspondence 


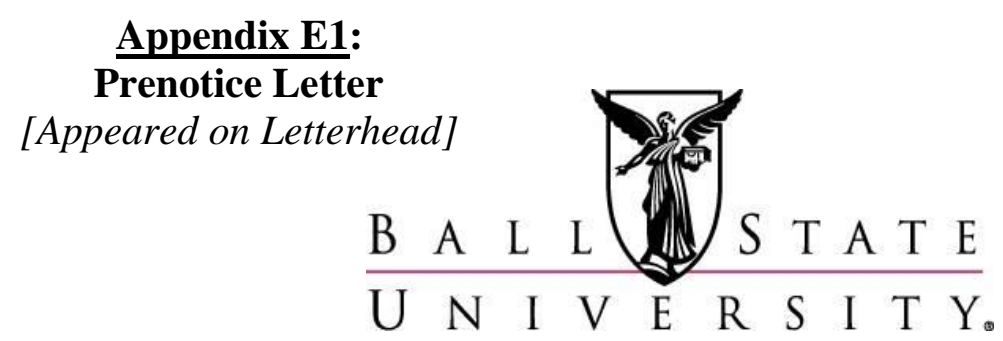

DEPARTMENT OF COUNSELING PSYCHOLOGY AND GUIDANCE SERVICES Muncie, Indiana 47306-0585 TEACHERS COLLEGE

Phone: 765-285-8040

July 22, 2008

Fax: 765-285-2067

Dr. Practicing Psychologist

123 Maple Dr.

Schenectady, NY 12345

Dr. Psychologist:

Greetings! I know you are very busy, but I wanted to take a moment to let you know about an upcoming study I am conducting, and to ask for your help.

My name is Kevin A. Harris, M.S., NCC. I am a doctoral candidate in the Department of Counseling Psychology and Guidance Services at Ball State University in Muncie, Indiana, and I am currently collecting data for my dissertation. Within the next week or so, you will receive a survey from me - a small packet of survey materials for you to fill out. I would greatly appreciate it if you would take the time to fill out these materials and return them to me in the return envelope which I will include in the survey packet. It should only take about half an hour to do this. As a token of my appreciation, $\$ 1.00$ in cash will be included in the survey packet. This is my way of thanking you for considering participating in my study. You are welcome to keep the $\$ 1.00$, whether or not you complete the survey. I know you must be very busy, but it is my hope that you will decide to participate.

The purpose of my dissertation is to examine a specific clinical judgment bias - to see whether the interaction of certain client factors and counselor characteristics leads to a hypothesized tendency for some clinicians to make more negative clinical judgments for certain clients. To investigate this hypothesis, I am giving psychologists who are in clinical practice vignettes to read and react to, as well as a series of six short questionnaires to complete. You are a part of a specially selected pool of psychologists that I would very much like to survey. This project has been approved by the Ball State University Institutional Review Board (IRB \#07-231). You are part of a national random sample of practicing psychologists, and in order to achieve results that are representative of American psychologists who are currently in clinical practice (as well as achieve the statistical power I need for my data analyses), it is important that most if not all of the psychologists in my sample complete this survey. I know that you are very busy and that you probably regularly receive requests to participate in research, but I would greatly appreciate it if you would take the time to complete this survey. You will be helping to contribute to an area of clinical judgment research that currently has very little research - and you will be helping me complete my dissertation.

Look to receive a packet of survey materials in the mail in the next week or so. If you know now that you DO NOT wish to participate, please e-mail me at kaharris@bsu.edu or call me at (765) 749-9774 and let me know. It is my hope, though, that you will choose to participate in this study. 
Thank you for your time.

Sincerely,

\section{Fomin a. Fania, MS, NCC}

Kevin A. Harris, M.S., NCC

Doctoral Candidate in Counseling Psychology

Ball State University

Muncie, Indiana
Kenin a. Fami, MS, NCC

Principal Researcher

Kevin A. Harris, M.S., NCC

Department of Counseling Psychology and Guidance Services

Ball State University

Muncie, IN 47306

Telephone: (765) 285-8040

E-mail: kaharris@bsu.edu

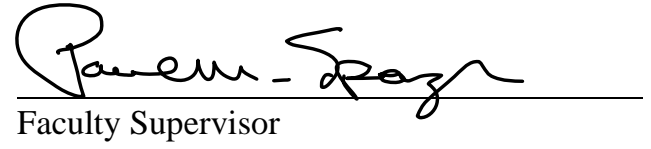

Dr. Paul M. Spengler, Ph.D., HSPP

Department of Counseling Psychology and Guidance Services

Ball State University

Muncie, IN 47306

Telephone: (765) 285-8040

E-mail: pspengle@bsu.edu 


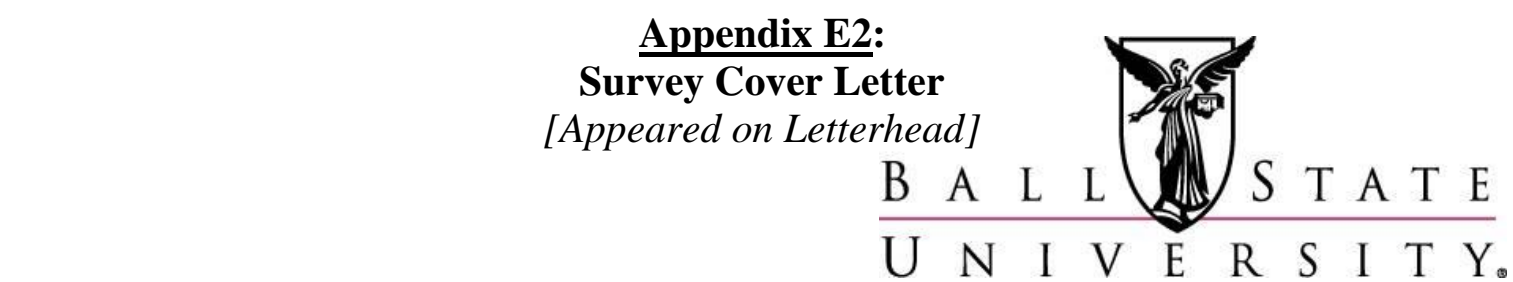

DEPARTMENT OF COUNSELING PSYCHOLOGY AND GUIDANCE SERVICES Muncie, Indiana 47306-0585 TEACHERS COLLEGE

Phone: $765-285-8040$

July 29, 2008

Fax: 765-285-2067

Dr. Practicing Psychologist

123 Maple Dr.

Schenectady, NY 12345

Dr. Psychologist:

Greetings! Thank you for taking the time to consider participating in this study. As I indicated in my letter to you that you should have received a week or so ago, you are a part of a specially selected pool of psychologists that I would very much like to survey. You are part of a national random sample of practicing psychologists that are representative of American psychologists currently in clinical practice. I know that you are very busy and that you probably regularly receive requests to participate in research, but I would greatly appreciate it if you would take the time to complete this survey. You will be helping to contribute to an area of clinical judgment research that currently has very little research - and you will be helping me complete my dissertation.

As I stated in my letter, my name is Kevin A. Harris, M.S., NCC. I am a doctoral candidate in the Department of Counseling Psychology and Guidance Services at Ball State University in Muncie, Indiana, and I am currently collecting data for my dissertation. The purpose of my dissertation is to examine a specific clinical judgment bias - to see whether the interaction of certain client factors and counselor characteristics leads to a hypothesized tendency for some clinicians to make more negative clinical judgments for certain clients. To investigate this hypothesis, I am giving psychologists who are in clinical practice vignettes to read and react to, as well as a series of six short instruments or questionnaires to complete.

Please take a few moments to fill out the enclosed materials and return them to me in the return envelope provided. It should only take about half an hour to do this. As a token of my appreciation, I have enclosed $\$ 1.00$ in cash to thank you for taking the time to consider participating in my study. You are welcome to keep the $\$ 1.00$, whether or not you complete the survey. Of course, it is my hope that you will decide to participate.

This project has been approved by the Ball State University Institutional Review Board (IRB \#07-231).

All of your information will be confidential. You will only be asked demographic information questions; no identifying information will be collected, apart from the provided return envelope which has a code number on it. This has only been labeled so that I can keep track of those who respond to this mailing; it is my intention to send a replacement questionnaire to nonrespondents in about a month, and I do not want to bother those who respond to this mailing promptly. As soon as I receive your completed materials, I will separate your return envelope from your survey materials, which will make your data anonymous.

The foreseeable risks or ill effects from participating in this study are minimal. You will be asked to read and make clinical judgments about a case vignette as well as fill out several questionnaires, but this is not anticipated to make you feel uncomfortable. If you do develop uncomfortable feelings during your participation in this research project, counseling services are available to you. In addition to the counseling services in your area that you already know about (but may not be appropriate for you to use because of your professional roles and potential dual relationship issues), you may search for counseling services on the internet by going to http://locator.apahelpcenter.org/, (Continued on the next page) Edition Date: 6/25/08 
www.nbcc.org/counselorfind2, http://therapistunlimited.com, www.google.com/Top/Health/Mental_Health/ Counseling_Services/, or www.yellowpages.com. You will be responsible for the costs of any care that is provided. It is understood that in the unlikely event of an injury or illness of any kind as a result of your participation in this research project that Ball State University, its agents and employees will assume whatever responsibility is required by law. If any injury or illness occurs in the course of your participation in this research project, please notify the principle investigator.

The direct benefits you will gain from your participation in this study are the $\$ 1.00$ in cash enclosed. Indirectly, you will also be making an important contribution to scientific learning. This study will contribute to the existing psychology of religion research literature because it will result in an empirical test of nine hypotheses (explained in detail on the debriefing website), representing an interaction of variables that has never before been examined, and using clinical vignettes that were generated by expert raters and mathematically selected through a magnitude estimation scaling procedure - vignettes which have never been used before, but which were pilot-tested and generated to more rigorous standards than similar vignettes in previous studies in this area. Thus your participation is important only through the time and effort of psychologists like you can I make this contribution to the existing psychology of religion research literature. So I thank you very much for your willingness to help.

Your participation in this study is completely voluntary and you are free to withdraw from the study at any time for any reason without penalty or prejudice. If you withdraw from the study, your data will not be used, and your materials will be destroyed. Please feel free to ask any questions at any time. You can contact me by e-mail at kaharris@bsu.edu, by telephone at (765) 749-9774, or by postal mail at Teachers College Room 622, Ball State University, Muncie, IN 47306.

For your rights as a research subject, the following person may be contacted: Coordinator of Research Compliance, Office of Academic Research and Sponsored Programs, Ball State University, Muncie, IN 47306, (765) 285-5070. You may keep this letter for future reference.

Again, thank you very much for your time. I sincerely appreciate it.

Cordially,

$$
\text { Fomi a. Fanio, MS, NCC }
$$

Kevin A. Harris, M.S., NCC

Doctoral Candidate in Counseling Psychology

Ball State University

Muncie, Indiana

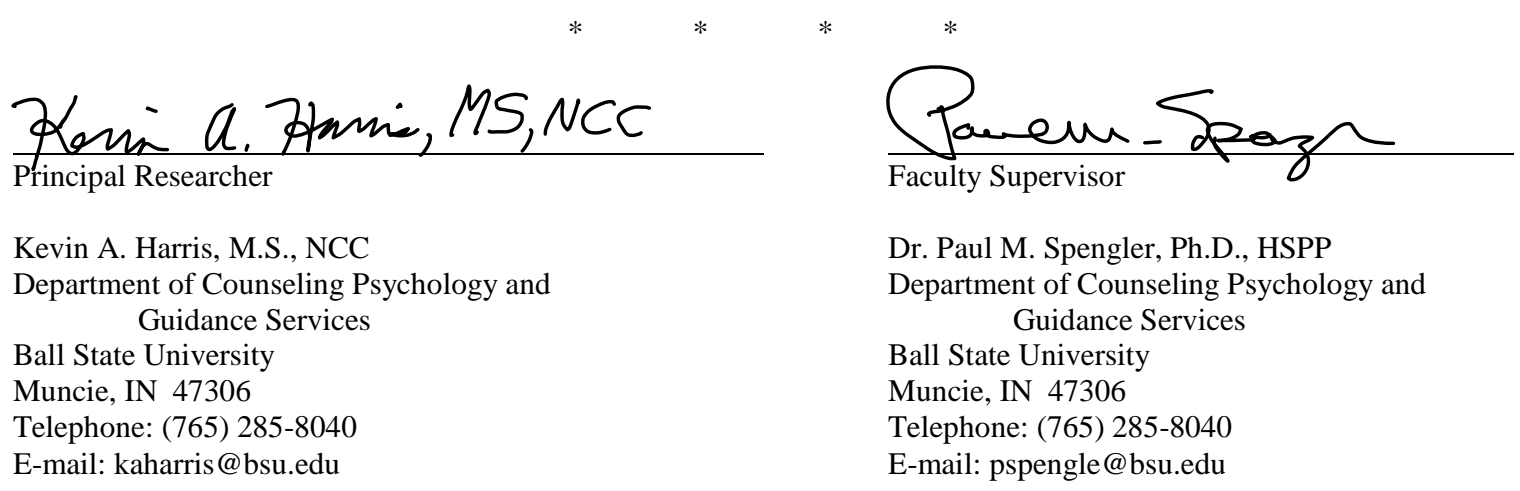

For full debriefing information, please visit the following website after you have completed the enclosed materials: http://kaharris.iweb.bsu.edu/cjfb.htm 


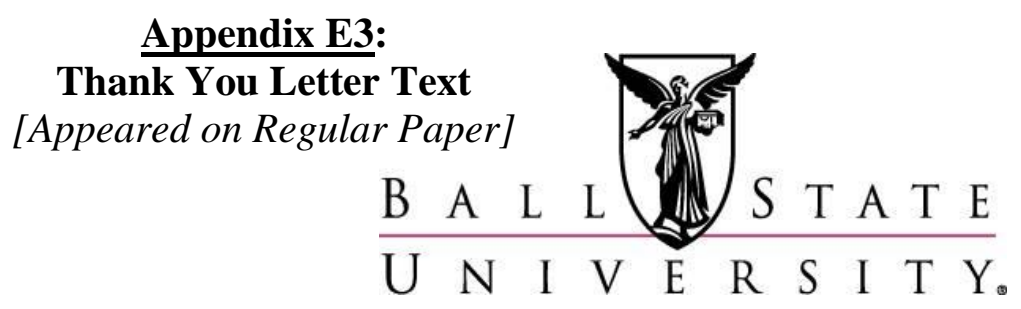

DEPARTMENT OF COUNSELING PSYCHOLOGY AND GUIDANCE SERVICES Muncie, Indiana 47306-0585

TEACHERS COLLEGE

Phone: $765-285-8040$

Fax: 765-285-2067

August 6, 2008

Dr. Practicing Psychologist

123 Maple Dr.

Schenectady, NY 12345

Dr. Psychologist:

By now, you should have received a survey packet in the mail from me: a survey of a national random sample of American psychologists currently in clinical practice, examining whether the interaction of certain client factors and counselor characteristics leads to a hypothesized tendency for some clinicians to make more negative clinical judgments for certain clients. I just wanted to take a moment to thank you for taking a little time out of your busy schedule to consider participating in this study. If you have already returned the survey packet, thank you very much. If you have not yet returned it, please do so in the next few days, whenever you get a chance.

Thanks!

Warmly,

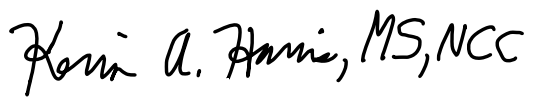

Kevin A. Harris, M.S., NCC

Doctoral Candidate in Counseling Psychology

Ball State University

Muncie, Indiana

P.S. If you did not receive this survey packet in the mail or if you have misplaced it, I would be happy to send you a replacement packet. You may contact me by postal mail at the return address on this postcard, by e-mail at kaharris@bsu.edu, or by telephone at (765) 749-9774. 


\section{Appendix E4: \\ Nonrespondent Mailing Cover Letter [Appeared on Letterhead] \begin{tabular}{lllllllllllll}
$B$ & B & $L$ & $L$ & $S$ & $T$ & $A$ & $T$ & $E$ \\
\hline$U$ & $N$ & $I$ & $V$ & $E$ & $R$ & $S$ & $I$ & $T$ & $Y$
\end{tabular}}

DEPARTMENT OF COUNSELING PSYCHOLOGY AND GUIDANCE SERVICES Muncie, Indiana 47306-0585 TEACHERS COLLEGE

Phone: 765-285-8040

Fax: 765-285-2067

August 18, 2008

Doctor:

Greetings again! A few weeks ago, I mailed you a survey packet for a study in which you were specially selected to participate. To the best of my knowledge you have not yet responded, so I am sending this replacement survey to you in the hopes that you will complete it. If you have already returned the earlier packet and I sent this to you by mistake (or our letters crossed in the mail), I apologize, and I thank you for your participation.

I earnestly hope you will consider participating in this study. As I indicated previously, you are a part of a specially selected pool of psychologists in clinical practice that I would very much like to survey. You are part of a national random sample of practicing psychologists that is representative of American psychologists currently in clinical practice. I know that you are very busy and that you probably regularly receive requests to participate in research, but I would greatly appreciate it if you would take the time to complete this survey. You will be helping to contribute to an area of clinical judgment research that currently has very little research - and you will be helping me complete my dissertation.

As I stated in an earlier letter, my name is Kevin A. Harris, M.S., NCC. I am a doctoral candidate in the Department of Counseling Psychology and Guidance Services at Ball State University in Muncie, Indiana, and I am currently collecting data for my dissertation. The purpose of my dissertation is to examine a specific clinical judgment bias - to see whether the interaction of certain client factors and counselor characteristics leads to a hypothesized tendency for some clinicians to make more negative clinical judgments for certain clients. To investigate this hypothesis, I am giving psychologists who are in clinical practice vignettes to read and react to, as well as a series of six short instruments or questionnaires to complete.

Please take a few moments to fill out the enclosed materials and return them to me in the return envelope provided. It should only take about half an hour to do this.

This project has been approved by the Ball State University Institutional Review Board (IRB \#07-231).

All of your information will be confidential. You will only be asked demographic information questions; no identifying information will be collected, apart from the provided return envelope which has your name and address on it. This has only been labeled so that I can keep track of those who respond to this mailing. As soon as I receive your completed materials, I will separate your return envelope from your survey materials, which will make your data anonymous.

The foreseeable risks or ill effects from participating in this study are minimal. You will be asked to read and make clinical judgments about a case vignette as well as fill out several questionnaires, but this is not anticipated to make you feel uncomfortable. If you do develop uncomfortable feelings during your participation in this research project, counseling services are available to you. In addition to the counseling services in your area that you already know 
about (but may not be appropriate for you to use because of your professional roles and potential dual relationship issues), you may search for counseling services on the internet by going to http://locator.apahelpcenter.org/, www.nbcc.org/counselorfind2, http://therapistunlimited.com, www.google.com/Top/Health/Mental_Health/ Counseling_Services/, or www.yellowpages.com. You will be responsible for the costs of any care that is provided. It is understood that in the unlikely event of an injury or illness of any kind as a result of your participation in this research project that Ball State University, its agents and employees will assume whatever responsibility is required by law. If any injury or illness occurs in the course of your participation in this research project, please notify the principle investigator.

The direct benefits you will gain from your participation in this study are the $\$ 1.00$ in cash which I enclosed in the original survey you received from me around a month ago. Indirectly, you will also be making an important contribution to scientific learning. This study will contribute to the existing psychology of religion research literature because it will result in an empirical test of nine hypotheses (explained in detail in the debriefing information), representing an interaction of variables that has never before been examined, and using clinical vignettes that were generated by expert raters and mathematically selected through a magnitude estimation scaling procedure - vignettes which have never been used before, but which were pilot-tested and generated to more rigorous standards than similar vignettes in previous studies in this area. Thus your participation is important - only through the time and effort of psychologists like you can I make this contribution to the existing psychology of religion research literature.

Your participation in this study is completely voluntary and you are free to withdraw from the study at any time for any reason without penalty or prejudice. If you withdraw from the study, your data will not be used, and your materials will be destroyed. Please feel free to ask any questions at any time. You can contact me by e-mail at kaharris@bsu.edu, by telephone at (765) 749-9774, or by postal mail at Teachers College Room 622, Ball State University, Muncie, IN 47306.

For your rights as a research subject, the following person may be contacted: Coordinator of Research Compliance, Office of Academic Research and Sponsored Programs, Ball State University, Muncie, IN 47306, (765) 285-5070. You may keep this letter for future reference.

Again, please seriously consider participating in this survey. I would very much appreciate it.

Sincerely,

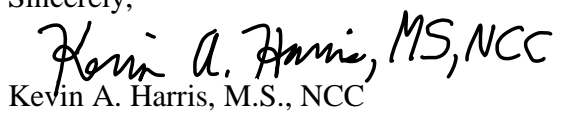

Doctoral Candidate in Counseling Psychology

Ball State University

Muncie, Indiana
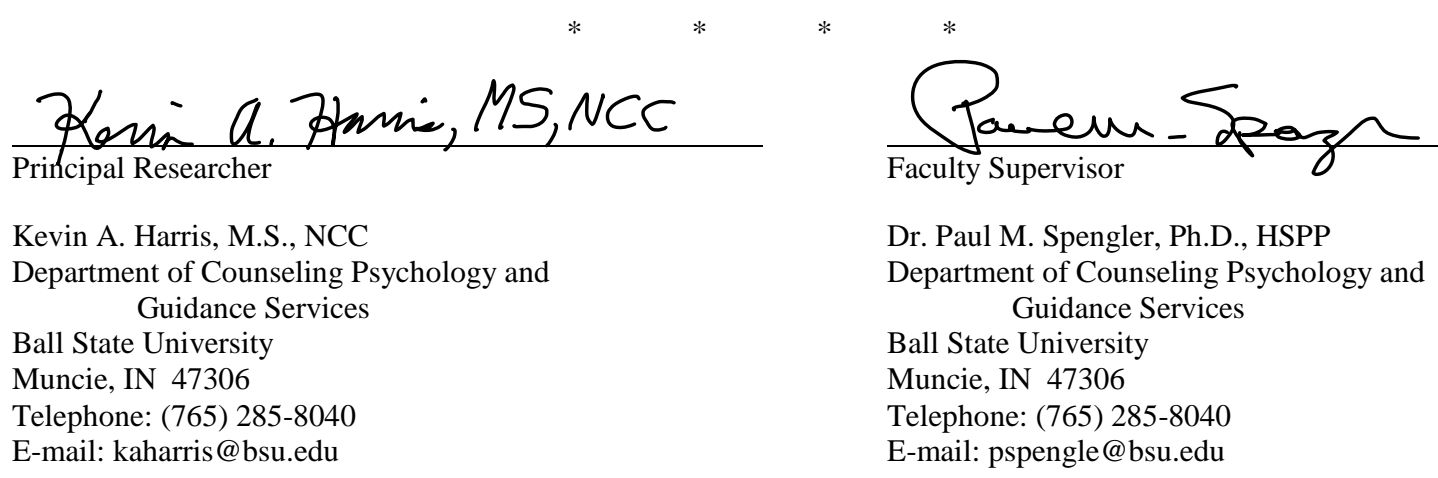

For full debriefing information, please visit the following website after you have completed the enclosed materials: http://kaharris.iweb.bsu.edu/cjfb.htm 


\section{Appendix E5: \\ Priority Mail Special Contact Letter \\ [Appeared on Letterhead]

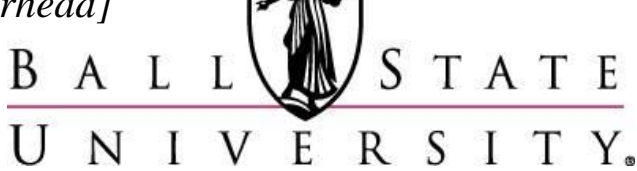

DEPARTMENT OF COUNSELING PSYCHOLOGY AND GUIDANCE SERVICES Muncie, Indiana 47306-0585

TEACHERS COLLEGE

Phone: 765-285-8040

Fax: 765-285-2067

September 12, 2008

Dr. Practicing Psychologist

123 Maple Dr.

Schenectady, NY 12345

Dr. Psychologist:

During the last few weeks, I have sent you several mailings about an important research study I am conducting as a doctoral candidate at Ball State University. Its purpose is to examine a specific clinical judgment bias - to see whether the interaction of certain client factors and counselor characteristics leads to a hypothesized tendency for some clinicians to make more negative clinical judgments for certain clients.

The study is drawing to a close, and this is the last contact that will be made with the random sample of psychologists in clinical practice which I have selected. I am sending this final contact by priority mail because of my concern that people who have not responded may have had different experiences than those who have. Hearing from everyone in this small national sample helps assure that the survey results are as accurate as possible.

I also want to assure you that your response to this study is voluntary, and if you prefer not to respond, that is fine. If you are not a psychologist in clinical practice and you feel that I made a mistake by including you in the random sample, please let me know by returning the blank questionnaire with a note indicating this. This would be very helpful.

I appreciate your willingness to consider my request as I conclude this effort to better understand a specific kind of bias in clinical decision-making. Thank you very much.

Sincerely,

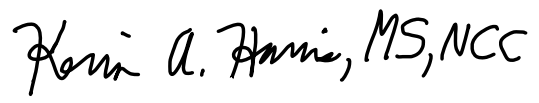

Kevin A. Harris, M.S., NCC

Doctoral Candidate in Counseling Psychology

Ball State University

Muncie, Indiana

P.S. If you did not receive this survey packet in the mail or if you have misplaced it, I would be happy to send you a replacement packet. You may contact me by postal mail at the return address on this letter, by email at kaharris@bsu.edu, or by telephone at (765) 749-9774. 


\section{Appendix F:}

Six Different Versions of Vignette Paragraph 2 Used in the Current Study

\section{High Religiousness}

MES R Vignette 6: $\psi=2.08$

RS Vignette 2: Mean Rating $=5.75$

Mary considers herself to be a born-again Christian and describes herself as "truly devoted to Jesus." She views Jesus as the source of her strength and happiness, both directly and indirectly. The church services she attends typically last 3 or 4 hours and involve singing, giving testimony, and praising Jesus. Privately, she prays and reads the Bible several times a day, decorates her home with religious items, and talks to others about what she believes. She volunteers two or three times a month at a local homeless shelter and often gives what little money she has to her church to support its overseas missions and foreign ministries, seeing her community involvement as "my privilege as a true Christian woman." She feels strongly that "it's not enough for me just to sit in the pew - I need to be involved in meeting the needs others have of clothing, shelter, food, and friendship in order to truly serve the Lord."

\section{Moderate Religiousness \\ MES R Vignette 9: $\psi=1.68$ \\ RS Vignette 9: Mean Rating $=4.19$}

Mary considers herself to be somewhat religious and describes her religion as important to her. While she considers herself to be a Christian, she thinks it is more important to live a good life than to be a member of a particular church, though she also believes church attendance to be important. She attends church services about twice a month, where she participates actively and listens attentively to the sermon. She spends time trying to grow in understanding of her faith and often reads books and magazines about her faith. She sometimes does charity work on the weekends, volunteering at a local homeless shelter, to which she also donates used clothing, furniture, and other household items when she no longer needs them. She occasionally tells others about her beliefs if they seem receptive, but she does not "preach" to others, not wanting to offend them. 


\section{Low Religiousness \\ MES R Vignette 14: $\psi=1.30$ \\ RS Vignette 11: Mean Rating = 3.13}

Mary considers herself to be a Christian and believes in Biblical teachings, but she also considers herself to be a skeptic who does not accept what she hears in regards to her religious beliefs without first questioning its validity. She describes attending church as something she occasionally does because it is "an important thing to do once in awhile," and because she believes it is important for her to learn occasionally from the lessons her pastor has to teach. She attends church about once a month. She prays to God sometimes, and she has read several passages from the Bible. Almost every year, around Christmas time, she volunteers at an annual soup kitchen which her church sponsors. She says that her beliefs seem to her to be true, but that she is not absolutely certain - "I could be wrong about what I believe."

\section{High Spirituality \\ MES S Vignette 6: $\psi=2.08$ \\ RS Vignette 18: Mean Rating = 5.56}

Mary considers herself to be a "mystical seeker" and describes herself as a very spiritual person whose close personal relationship with the universe influences every area of her life. She states that her faith is more than just a belief system - it is a fundamental part of her individual identity, family life, and cultural upbringing. She typically worships by attending mantra workshops - chant rituals that last for half a day or more - noting that at times this experience is incredibly powerful for her. During the rest of the week, she often privately talks to a higher power to make sure her life is on track. She regularly gives a generous percentage of her income to local charities, and just last year she donated an additional $\$ 2000$ towards the purchase of a new stove for a nearby homeless shelter. She often talks to others about what she believes, and makes it a point to tell others what an active role that her personal relationship with the universe has taken in guiding her life since she "realized the truth of the universe." 


\section{Moderate Spirituality}

MES S Vignette 4: $\psi=1.70$

RS Vignette 26: Mean Rating $=5.67$

Mary considers herself to be a seeker and describes herself as a somewhat spiritual person who believes in the divine. She feels a sense of trust and security because she has a general feeling that "the universe provides." She attends spiritual healing services occasionally, and, when she does, she often feels the presence of the divine with her. She prefers to worship quietly and privately, by praying to a divine power a few times a week and occasionally reading books about religion or spirituality. She faithfully gives a percentage of each of her paychecks to the United Way. She refers to her beliefs as feeling right for her but says she can understand why other people might believe differently than she does.

\section{Low Spirituality \\ MES S Vignette 14: $\psi=1.26$ \\ RS Vignette 31: Mean Rating $=4.33$}

Mary considers herself to be "not very spiritual - I just believe in some kind of a higher power." She describes her faith as something which used to be important to her but which no longer has much relevance to her life any more. She attends meditation classes 2 or 3 times each month. She prays to a higher power sometimes, and she has read passages from several religious texts. She volunteers at a local homeless shelter around Thanksgiving almost every year. She tells people about her spiritual beliefs if they ask her, but she also believes everyone is entitled to their own beliefs and should live their lives as they choose. 


\section{Appendix G:}

Vignette Development Procedure

Six versions of a case vignette were created to serve as the treatment condition in the current study (see Appendix D1 and Appendix F). This vignette describes a client with Generalized Anxiety Disorder (GAD) and allows two independent variables to be manipulated: client faith type and client faith magnitude. Three versions depict a client with high, moderate, or low religiousness, while three versions depict a client with high, moderate, or low spirituality. This vignette is based on Hillowe's (1985) Religious Mary and Nonreligious Mary vignettes, Feeser's (1997) Spiritual Mary vignette, Minyard's (1983) religious cues, and items from several measures of religiousness and spirituality, including the Religious Values Scale (Morrow, Worthington, \& McCullough, 1993), the Religious Orientation Scale (Allport \& Ross, 1967; Gorsuch \& McPherson, 1989; Gorsuch \& Venable, 1983), the Religious Belief Inventory (Lee, 1965), the Attitude Toward Evangelism Scale (Seyfarth et al., 1984), the Omnibus Personality Inventory Religious Orientation Scale (Heist \& Yonge, 1968), the Index of Core Spiritual Experiences (Kass, Friedman, Lesserman, Zuttermeister, \& Benson, 1991), the Mysticism Scale (Hood, 1975), the Spiritual Assessment Inventory (Hall \& Edwards, 1996), and the Spiritual Well-Being Questionnaire (Moberg, 1984).

\section{Previous Research}

Previously, similar studies used clinical analogue experiments. Wadsworth and Checketts (1980) varied the religious affiliation that was stated in written case studies and gave ambiguous descriptions that allowed for more than one possible diagnosis for the 
sake of making any bias that existed appear more obvious. On the other hand, Lewis and Lewis (1985) gave descriptions that clearly only fit one diagnosis. Hillowe (1985) created written case vignettes "indicating moderate religious belief and behavior as a patient might report them rather than simply stating an affiliation" and "combining the information needed for diagnosis and prognosis that is commonly gained from an initial intake interview or report with the clarity and cohesiveness of a narrative vignette" ( $p$. 59). Hillowe (1985) created Religious Mary and Nonreligious Mary vignettes describing a client with Obsessive Compulsive Neurosis and Avoidant Personality Disorder based on portrayals of real clients from the DSM-III Casebook (Spitzer, Skodol, Gibbon, \& Williams, 1981). Hillowe (1985) intended for the religious vignette to portray a moderately religious client, to sufficiently differentiate it from the nonreligious vignette and to avoid the negative stigma of extreme religiousness. As a manipulation check, Hillowe (1985) asked graduate students to rate on Likert-type scales ranging from 1 to 7 the extent of religious belief, the intensity of religious belief, the degree of religious involvement, and the overall importance of religion in the depicted person's life. For each vignette, an overall religiousness score was calculated by averaging the scores from all questions and from all the students who rated that vignette. In this way, Religious Mary was given a mean religiousness score of 5.035, indicating moderate religiousness, while Nonreligious Mary was given a mean religiousness score of 1.29, indicating low religiousness.

In subsequent studies, Shafranske and Malony (1990) and Feeser (1997) integrated Hillowe's (1985) Religious and Nonreligious Mary vignettes into their experiments. Feeser (1997) modified the vignettes to be consistent with DSM-IV criteria, 
adding a statement that the client viewed herself as socially inept ("unpopular"). Additionally, Feeser (1997) developed another version of these vignettes which depicted the same client as spiritual. In developing this new Spiritual Mary vignette, references to a belief in God and God's plan, a religious congregation, and a Bible study group were replaced with references to "a feeling that 'the universe provides'," "a group which considers issues of personal spirituality as they relate to daily life," and a "meditation and yoga class" (Feeser, 1997, p. 156). Otherwise, Feeser's (1997) Spiritual Mary vignette was identical to Hillowe's (1985) Religious Mary vignette. Unlike Hillowe (1985), though, Feeser (1997) did not include a manipulation check.

In an older, particularly well-constructed study, Minyard (1983) investigated how clinicians responded to a number of religious cues within a series of vignettes. Each vignette contained three religious cues, or references to church attendance, frequency of prayer, and evangelism, and each of the three cues was written at three different levels to represent low, medium, and high levels of these cues. The cues were written based on "a lengthy process of interviews with clergy, a review of the literature, and discussion among a group of interested students and faculty" (Minyard, 1983, p. 92), as well as drawn from a previous study (Sullivan, Pargament, McGath, Echemendia, Minyard, Manges, Gibson, Cook, Bena, Kennell, Goldblatt, \& Pritchard, 1982). Church attendance was chosen as a cue because of the number of studies that use frequency of church attendance as a measure of religiousness, and because Sullivan et al. (1982) found that $59 \%$ of participants used church attendance to a significant degree to make clinical judgments. Frequency of prayer was chosen as a cue because it served as a reasonable indicator of personal practice, while evangelism was chosen because it was an indication 
of public behavior and served as "a more vigorous public display of religious commitment” (Minyard, 1983, p. 94). Participants sorted cues into categories and rated on a 9-point Likert-type scale the degree to which each cue represented its intended dimension. The cues were then incorporated into vignettes - three cues per vignette, one each from the categories of church attendance, frequency of prayer, and evangelism, and each cue at systematically varying levels (low church attendance, low prayer frequency, low evangelism (LLL); low, low, medium (LLM); LLH; LML; LMM; etc.), yielding 27 possible combinations:

This combination of the three religiosity cues into a single cue was done so that implausible combinations (e.g., the combination of a "low" level of church attendance and a "low" level of personal practice with a "high" level of evangelism (LLH) which would have read "Joe rarely attends church services or prays alone but enthusiastically promotes his religious beliefs to others whenever possible.") would not occur in the profiles. (Minyard, 1983, p. 106)

This yielded 22 actual combinations. Participants then rated the degree of religiousness and the degree of pathology of each case.

\section{The Current Vignette}

The vignette in the current study is about a fictional client named Mary and contains two paragraphs: an initial paragraph describing Mary’s presenting problem, Generalized Anxiety Disorder (GAD), and a second paragraph describing Mary’s religiousness or spirituality. GAD was chosen as the presenting problem because of the previous research (i.e., Gartner et al., 1990a, 1990b) suggesting that very religious clients with anxiety symptoms are more likely to be diagnosed with OCD and less likely to be 
diagnosed with GAD than less religious clients. Unlike Hillowe's (1985) vignettes, which maintained a constant level of (moderate) religiousness while including a latitude of diagnoses, the different versions of the vignette in this study include a latitude of different magnitudes of religiousness and spirituality (so that magnitude of client faith could be manipulated) while maintaining a single diagnosis across all versions (to decrease variability in the dependent measure and hold diagnosis constant).

\section{Pilot Study 1: Diagnosis Content Validation}

The first paragraph of the vignette gives basic demographic information for Mary and describes her symptoms of GAD. The content of this paragraph was drawn primarily from Feeser's (1997) Religious Mary vignette, but since this vignette was developed to represent a client with Obsessive-Compulsive Disorder and Avoidant Personality Disorder, all references to symptoms of Avoidant Personality Disorder were removed from the vignette, and two phrases were added to the remaining sentences to more clearly represent a client with general symptoms of anxiety cutting across multiple situations that is, a client with GAD and not OCD or another anxiety disorder. To the sentence "She now spends hours each night rehashing the day's events, especially interactions with her professors and exchanges with other students in a particularly demanding course she is taking" was added the clause "but also sometimes interactions with her parents, with whom she finds it difficult to get along," to illustrate that her worries are about everyday or real-life problems and to better demonstrate that she is worried about a number of events. The sentence "She also does this at night when she is supposed to be sleeping, so she has not been sleeping well lately and finds herself unusually irritable and easily fatigued" was also added, to further demonstrate the pervasiveness of her worries and to 
note that her worries are accompanied by physical symptoms. The content was also slightly altered to make Mary younger - 20 years old, which the Diagnostic and Statistical Manual of Mental Disorders indicates is not an unusual age for the onset of GAD (DSM-IV-TR, 2000). To control for extraneous variables and ensure that participants responded to these vignettes only on the basis of the religiousness or spirituality they portrayed, all versions of the vignette were identical with respect to race, gender, age, educational level, referral status, affect, presenting concerns, and level of functioning. This was achieved by keeping the initial paragraph the same in each version of the vignette:

Mary is a 20-year-old, Caucasian female who is an undergraduate student at a local university. She is self-referred. She appeared mildly apprehensive during the initial interview and seemed embarrassed about her problems. Her presenting complaint is that she is having difficulty reading, studying, and concentrating because she has become increasingly preoccupied with thoughts she cannot dispel. She now spends hours each night rehashing the day's events, especially interactions with her professors and exchanges with other students in a particularly demanding course she is taking, but also sometimes interactions with her parents, with whom she finds it difficult to get along. She says that she endlessly tries to correct in her mind any mistakes she might have made. She runs over every event, asking herself if she behaved properly or said the right thing. She does this at her desk when she is supposed to be reading, doing homework, or working on her undergraduate thesis, which she has nearly completed. It is not unusual for her to look at the clock after such a period of rumination and find, to 
her surprise, that two or three hours have elapsed. She also does this at night when she is supposed to be sleeping, so she has not been sleeping well lately and finds herself unusually irritable and easily fatigued.

To ensure that the initial paragraph of the vignette accurately portrays a client presenting with GAD, a manipulation check was conducted. Fourteen psychologists in clinical practice were given this paragraph and asked to give this person a diagnosis or diagnoses based on the information given. They were also asked to indicate on a 7-point Likert-type scale ranging from $1=$ Not Very Accurately to $7=$ Very Accurately how well this paragraph depicted a person whose primary presenting concern was Generalized Anxiety Disorder, as well as how well this paragraph depicted a person with ObsessiveCompulsive Disorder and eight other anxiety disorder diagnoses (Panic Disorder with or without Agoraphobia, Agoraphobia without history of Panic Disorder, Specific Phobia, Social Phobia, Posttraumatic Stress Disorder, Acute Distress Disorder, Adjustment Disorder with Anxiety or with Mixed Anxiety and Depressed Mood, or any other Anxiety Disorder not listed previously). The questionnaire given to these 14 psychologists in Pilot Study 1 appears in Appendix H. Eleven of the 14 psychologists diagnosed the client described with GAD, with the remaining three psychologists diagnosing the client with another anxiety disorder. These 14 psychologists rated GAD as the most accurate diagnosis, giving it an average accuracy rating of 6.64 - more than twice the accuracy rating of the diagnosis rated next most accurate $(\mathrm{OCD}=2.93)$. The data from this pilot study are shown in Table 11. 
Table 11

Psychologist Expert Raters Diagnostic Ratings

Variable

$N \quad \% *$

$M \quad S D$

Diagnosis:

$\begin{array}{lcc}\text { Generalized Anxiety Disorder } & 11 & 78.6 \% \\ \text { R/O GAD } & 1 & 7.1 \% \\ \text { Obsessive Compulsive Disorder } & 1 & 7.1 \% \\ \text { R/O OCD } & 2 & 14.3 \% \\ \text { Anxiety Disorder NOS } & 2 & 14.3 \% \\ \text { R/O Major Depressive Disorder } & 1 & 7.1 \%\end{array}$

How accurately does this paragraph depict:

Disorder of Childhood?

$1.36 \quad 0.84$

Substance Disorder?

$1.07 \quad 0.27$

Psychotic Disorder?

$1.14 \quad 0.36$

Mood Disorder?

$3.07 \quad 1.49$

Anxiety Disorder?

$6.64 \quad 0.63$

Somatoform Disorder?

$1.29 \quad 0.61$

Adjustment Disorder?

$2.00 \quad 1.15$

V-Code?

$1.79 \quad 1.12$

No Diagnosis?

$1.21 \quad 0.58$

* Total percentages do not equal $100 \%$ due to missing or multiple responses 
Table 11, Continued

Psychologist Expert Raters Diagnostic Ratings

\begin{tabular}{|c|c|c|c|c|}
\hline Variable & $N$ & $\% *$ & $M$ & $S D$ \\
\hline \multicolumn{5}{|l|}{ Other: } \\
\hline Perfectionism & 1 & $7.1 \%$ & & \\
\hline Social Anxiety Disorder & 1 & $7.1 \%$ & & \\
\hline Panic Disorder? & & & 1.93 & 1.59 \\
\hline Agoraphobia? & & & 1.43 & 0.65 \\
\hline Obsessive Compulsive Disorder? & & & 2.93 & 1.86 \\
\hline Generalized Anxiety Disorder? & & & 6.64 & 0.50 \\
\hline Post-Traumatic Stress Disorder? & & & 1.50 & 0.85 \\
\hline Social Phobia? & & & 2.79 & 1.42 \\
\hline Other Anxiety Disorder? & & & 2.57 & 1.74 \\
\hline
\end{tabular}

* Total percentages do not equal $100 \%$ due to missing or multiple responses

Pilot Study 2: Faith Paragraph Development and Content Validation

The second paragraph in the vignette describes Mary's magnitude of faith and represents the treatment or experimental manipulation that was conducted in the current study. Six versions of this paragraph were developed: High, Moderate, and Low Religiousness versions as well as High, Moderate, and Low Spirituality versions. The six versions of the second paragraph were developed in four stages: (a) development of two pools of potential vignette versions, (b) content validation, (c) magnitude estimation scaling of the level of religiousness or spirituality in the vignette versions, and (d) 
vignette version selection. This four-stage process of vignette development was based on a procedure outlined in Lodge (1981) and Spengler, Blustein, and Strohmer (1990). Stages (a) and (b) took place in Pilot Study 2. Stages (c) and (d) took place in Pilot Study 3.

In order to develop two pools of potential vignette versions, 37 versions of the second paragraph were written using a 6-sentence standard format: a pool of 16 religious versions and a second pool of 21 spiritual versions. Each of the 37 vignette versions contains six sentences in which six different qualities are systematically varied from high to low: (1) a cognitive statement of faith, (2) a personal statement of faith, (3) a public expression of faith, (4) a private expression of faith, (5) charitable involvement, and (6) evangelism/openness. The first sentence contains a cognitive statement of faith - a statement describing Mary's religious or spiritual worldview. The second sentence is a personal statement of faith, a description of something specific that Mary believes, with content appropriate to a specific denomination or spiritual belief system. The third sentence depicts public expressions of Mary's faith - attending church or spiritual events and the nature of the rituals she performs, while the fourth sentence illustrates private expressions of Mary's faith - the private rituals she participates in, such as prayer, meditation, and reading the Bible or other religious or spiritual texts. The final two sentences of the paragraph depict Mary's social expressions of faith. The fifth sentence discusses Mary's involvement in charity, while the sixth sentence describes her evangelism (preaching behavior) and her openness to other viewpoints or worldviews that differ from her own. These six content areas were drawn primarily from Hillowe's (1985) Religious Mary and Nonreligious Mary vignettes, Feeser’s (1997) Spiritual Mary 
vignette, Minyard's (1983) religious cues, and the Religious Values Scale (Morrow et al., 1993). In fact, the wording of many of the sentences was drawn directly from these sources.

The 16 religious vignette versions were originally intended to portray a general, nonspecific form of Protestant Christianity that could represent a wide variety of denominations. After consulting with an expert on clinical judgment research, however, it was decided that the versions would be written to portray one specific Christian denomination, to decrease the variability in the stimulus vignettes and to yield more accurately portrayals of actual real-world religious practice (rather than less accurate portrayals of hypothetical general religious practice). A denomination was sought that included a wide variety of beliefs and behaviors, ranging from high to low religiousness and from conservative to liberal expressions of this religiousness, as well as a variety of different individuals and churches from different cultures and regions within the United States. Also, a denomination was sought whose characteristic beliefs and practices were similar to the beliefs and practices of other Christian denominations; denominations whose characteristic beliefs or practices were specific to that denomination were excluded. Baptist Christianity, the second largest Christian denomination in the United States (Richards \& Bergin, 2000), was therefore chosen as the specific denomination to portray in the vignettes, because it met the aforementioned criteria. (Catholicism, the largest Christian denomination in the United States and the world, was not selected because of the number of denomination-specific beliefs and practices that characterize this belief system, such as the teachings of the Catechism, parish structure, deference to the papacy and the authority of the Vatican, the emphasis on the Eucharist, and a special 
reverence for the Virgin Mary; Richards \& Bergin, 2000.) The 16 religious vignette versions are presented in Appendix I.

The 21 spiritual vignette versions were generated by first reviewing recent research on the similarities and differences between the constructs of religiousness and spirituality (e.g., Cook et al., 2000; Hill et al., 2000; Zinnbauer \& Pargament, 2005) and then rewriting the religious vignettes to reflect spiritual content. The following operational definitions of religiousness and spirituality were adopted from Hill et al. (2000):

Religiousness is a search for the sacred (or non-sacred things such as social contact or appearing virtuous to your neighbors) in a traditional institutional context that typically facilitates searches for the sacred, and Spirituality is a personal search for the sacred which may or may not occur in a traditional institutional context that typically facilitates searches for the sacred, where

The sacred is an ultimate, transcendent, or divine being, reality, or truth as perceived by an individual.

In order to generate spiritual versions that were clearly different from the religious versions, the Hill et al. (2000) definition of spirituality was modified slightly, and spirituality was operationalized as a personal search for the sacred which does not occur in a traditional institutional context. Sentence by sentence, each religious vignette version was rewritten so that the same six qualities that are systematically varied from high to low in the religious versions (a cognitive statement, a personal statement, a public expression, a private expression, charitable involvement, and evangelism/openness) are 
also varied the same way in the spiritual versions. The religious content of each sentence was modified to reflect spiritual content, while maintaining the same quality as the original religious sentence had. For example, the religious personal statement "Mary considers herself to be a very devout Christian and describes herself as a person whose entire life revolves around Jesus" was rewritten into a spiritual personal statement that read "Mary considers herself to be very spiritual and describes herself as a person whose entire life revolves around her relationship with the universe." Similarly, the religious private expression of faith stating "She prays to God several times a day and usually reads the Bible for an hour or two each day" was rewritten into a spiritual private expression of faith reading "She prays to a higher power several times a day and usually meditates for an hour or two each day." In this way, the religious vignette versions were rewritten with spiritual content while maintaining their original six qualities. Because of the subjectivity of this rewriting task, the differing opinions on how (if at all) spirituality differs from religiousness (c.f. Hill et al., 2000; Zinnbauer \& Pargament, 2005), and the relative lack of spiritual vignettes in previous research, a greater number of spiritual vignette versions were written so that if some of them were inaccurate representations of a spiritual-but-not-religious person, several of these versions could be dropped without the vignette pool becoming too small. The 21 spiritual vignette versions are presented in Appendix I.

To evaluate the content validity of the two vignette pools that were written, the 16 religious vignette versions and 21 spiritual vignette versions were reviewed by a series of expert raters. First, the vignette versions were edited by two graduate professors: a clinical judgment expert and a psychology of religion expert (both members of the 
primary researcher's dissertation committee), who reviewed the vignette versions for content and gave feedback on how they should be rewritten to more accurately represent varying levels of religiousness and spirituality. Next, the rewritten vignette versions were given to a group of religious expert raters, who rated on 7-point Likert-type scales ranging from $1=$ Not Very Accurately to $7=$ Very Accurately how well each vignette version depicted a religious person or a spiritual person. Baptist ministers and Unitarian Universalist pastors in Muncie, Indiana and south Florida and Religious Studies professors at several Indiana and Miami universities were approached by e-mail and asked for their help because of their expertise in matters of religion and spirituality Baptist ministers because of their specific knowledge of Baptist Christianity, Unitarian Universalist pastors because of their specific knowledge of nondenominational or crossdenominational spirituality, and Religious Studies professors because of their specific knowledge of the theoretical and practical distinctions and overlap between religious and spiritual expression. The questionnaire given to these religious expert raters appears in Appendix J. A total of 11 religious expert raters returned completed questionnaires. Their mean accuracy ratings are displayed in Table 12. The 13 religious vignette versions and 13 spiritual vignette versions with the highest mean accuracy ratings were selected for further use and rewritten slightly to more accurately depict varying levels of religiousness and spirituality, based on the feedback from the religious expert raters. 
Table 12

Mean Accuracy Ratings of 37 Vignette Versions

\begin{tabular}{|c|c|c|c|c|c|}
\hline Vignette & Religious & Spiritual & Vignette & Religious & Spiritual \\
\hline Version & Person & Person & Version & Person & Person \\
\hline 1 & 5.75 & 1.88 & 20 & 5.78 & 1.25 \\
\hline 2 & 5.75 & 2.00 & 21 & 6.44 & 1.63 \\
\hline 3 & 5.63 & 1.63 & 22 & 6.33 & 1.50 \\
\hline 4 & 5.88 & 1.63 & 23 & 5.89 & 1.50 \\
\hline 5 & 5.25 & 2.13 & 24 & 6.44 & 1.88 \\
\hline 6 & 5.13 & 2.63 & 25 & 6.11 & 2.00 \\
\hline 7 & 5.63 & 1.75 & 26 & 5.67 & 1.50 \\
\hline 8 & 3.50 & 4.00 & 27 & 5.44 & 1.63 \\
\hline 9 & 4.19 & 3.50 & 28 & 5.11 & 1.38 \\
\hline 10 & 3.88 & 3.38 & 29 & 5.67 & 1.38 \\
\hline 11 & 3.13 & 3.88 & 30 & 5.11 & 1.38 \\
\hline 12 & 3.13 & 4.50 & 31 & 4.33 & 1.50 \\
\hline 13 & 2.88 & 3.63 & 32 & 4.78 & 1.50 \\
\hline 14 & 2.13 & 3.25 & 33 & 3.78 & 1.50 \\
\hline 15 & 2.25 & 2.75 & 34 & 3.89 & 1.75 \\
\hline 16 & 6.44 & 1.50 & 35 & 3.25 & 1.14 \\
\hline 17 & 6.39 & 1.75 & 36 & 2.75 & 1.14 \\
\hline 18 & 5.56 & 1.50 & 37 & 7.00 & 1.20 \\
\hline 19 & 5.22 & 1.38 & & & \\
\hline
\end{tabular}




\section{Pilot Study 3: Magnitude Estimation Scaling}

Following content validation, the magnitude or level of religiousness or spirituality in each rewritten vignette version was established using a magnitude estimation scaling (Lodge, 1981) task based on Tanenhaus and Murphy (1981) and Spengler, Blustein, and Strohmer (1990). Undergraduate college students were selected to complete the magnitude estimation scaling task for two reasons: (1) in order to increase the generalizability of the vignette versions, adding a third sample of people (in addition to psychologists and religious experts) who validated the versions; and (2) in an effort to assess magnitude of religiousness and spirituality as perceived by the (welleducated) general population rather than as perceived by psychologists or religious experts, who because of their specialized knowledge might view magnitude of religiousness and spirituality different than ordinary lay persons. First, 24 undergraduate college students completed a cross modality matching task, which served as a mathematical correction for the regression bias that typically occurs in the magnitude estimation scaling of social stimuli. The students were asked to assign number estimates to the length of 12 lines $(26,74,4,98,38,2,14,18,64,86,150$, and $200 \mathrm{~mm}$ in length), with a labeled $50 \mathrm{~mm}$ reference line provided for comparison. The students also performed a similar task by drawing lines to match the length of provided numbers (26, $74,4,98,38,2,14,18,64,86,150$, and 200 ), again with a labeled $50 \mathrm{~mm}$ reference line provided for comparison. Second, students were given a reference vignette describing a moderately religious client, which was paired with an assigned number of 50 and a 50 $\mathrm{mm}$ reference line. Students were then given 13 of the religious vignette versions with the highest accuracy ratings and asked first to assign a number estimate and then to draw a 
line representing the magnitude of religiousness described by each vignette version. A control vignette version describing a person who was very mathematical was imbedded among the 13 religious vignette versions, to provide a reference vignette version with no religious content. This served as a manipulation check, to ensure that participants were rating the magnitude of religiousness in the vignette and not some other characteristic (and to check if participants were paying attention). The questionnaire given to these 24 undergraduate college students appears in Appendix K. One participant returned an incomplete questionnaire, so this person's data was dropped.

Similarly, a separate group of 23 students were given 13 of the spiritual vignette versions with the highest accuracy ratings and asked to assign number estimates and draw lines for these vignette versions. Again, a control mathematics vignette version was imbedded among the 13 spiritual vignette versions as a reference vignette version. The questionnaire given to these 23 undergraduate college students also appears in Appendix K. One participant in this group returned an incomplete questionnaire as well, so this person's data was dropped.

Afterwards, magnitude estimation scale values were calculated for the level of religiousness and spirituality of the vignette versions by calculating the geometric means for both the number estimates and the length of the lines that participants drew in response to the vignette versions. These geometric means were then corrected for regression bias by raising each one by the inverse of the slope obtained by calculating the geometric means for both the number estimates and the length of the lines that participants initially drew in response to the given lines and numbers. Finally, a magnitude estimation scale value was calculated for each vignette version by computing 
the square root of the product of the adjusted number-estimate and line-length means. The results of these calculations are displayed in Table 13.

Following these mathematical procedures, six vignette versions were selected to represent high, moderate, and low religiousness and spirituality, based on their magnitude estimation scale (MES) values and uncorrected mean magnitude scores. Vignette 9 with a MES value of 1.68 (Uncorrected Mean = 59, Mean Accuracy Rating $=4.19)$ was selected to represent moderate religiousness, and Vignette 26 with a MES value of 1.70 $($ Uncorrected Mean $=53$, Mean Accuracy Rating $=5.67)$ was selected to represent moderate spirituality. Four more vignettes were then selected representing approximately half and twice the degree of religiousness and spirituality as the moderate vignettes represented. Vignette 2 with a MES value of 2.08 (Uncorrected Mean $=129$, Mean Accuracy Rating $=5.75)$ was selected to represent high religiousness, and Vignette 11 with a MES value of $1.30($ Uncorrected Mean $=27$, Mean Accuracy Rating $=3.13)$ was selected to represent low religiousness. Vignette 18 with a MES value of 2.08 $($ Uncorrected Mean $=128$, Mean Accuracy Rating $=5.56)$ was selected to represent high spirituality, and Vignette 31 with a MES value of 1.26 (Uncorrected Mean =24, Mean Accuracy Rating $=4.33$ ) was selected to represent low spirituality. These vignettes represent low, moderate, and high levels of client religiousness and spirituality in the current study. This procedure enabled client faith magnitude - both religiousness and spirituality - to be treated as continuous (ratio) variables. 
Table 13

Magnitude Estimation Scaling Calculations

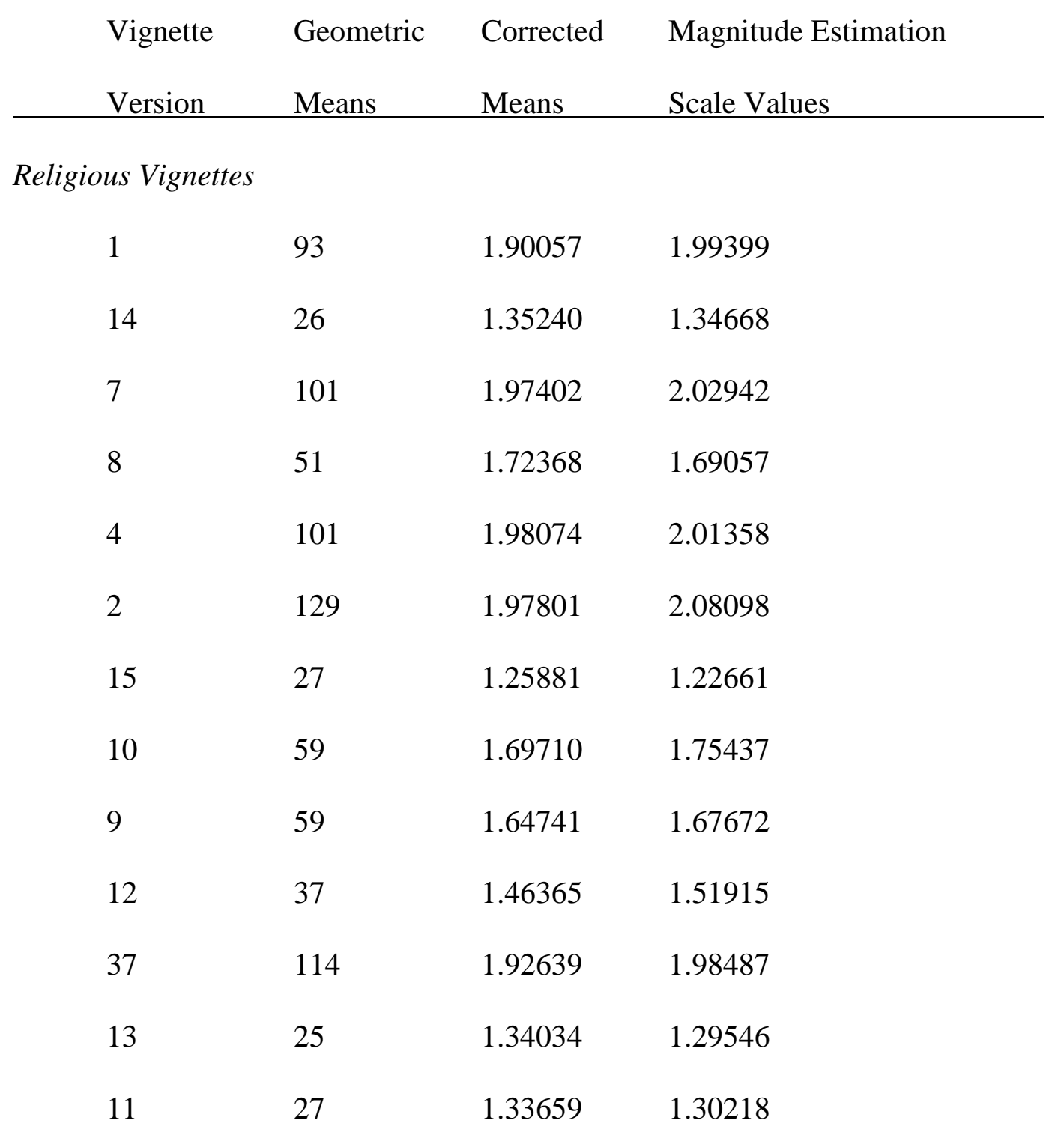

Spiritual Vignettes

\begin{tabular}{llll}
17 & 92 & 1.92114 & 1.96035 \\
35 & 22 & 1.31072 & 1.24937 \\
24 & 82 & 1.87120 & 1.89040 \\
26 & 53 & 1.68677 & 1.69824 \\
\hline
\end{tabular}


Table 13, Continued

Magnitude Estimation Scaling Calculations

\begin{tabular}{cccc}
$\begin{array}{c}\text { Vignette } \\
\text { Version }\end{array}$ & Geometric & Corrected & $\begin{array}{l}\text { Magnitude Estimation } \\
\text { Scale Values }\end{array}$ \\
\hline Spiritual Vignettes, & Continued & & \\
21 & 98 & 1.89146 & 1.95373 \\
18 & 128 & 2.03251 & 2.08397 \\
36 & 16 & 1.19951 & 1.05139 \\
29 & 46 & 1.63848 & 1.64498 \\
27 & 55 & 1.69038 & 1.70566 \\
32 & 30 & 1.44526 & 1.43274 \\
20 & 88 & 1.83045 & 1.88864 \\
34 & 38 & 1.46398 & 1.47642 \\
31 & 24 & 1.22987 & 1.25803 \\
\hline
\end{tabular}




\section{Appendix H:}

Pilot Study 1 Questionnaire

\section{Clinician Questionnaire}

\section{Please read the following description of Mary, a fictional client, and answer the questions that follow. Please answer the questions as they appear and do not read ahead.}

Mary is a 20-year-old female who is an undergraduate student. She was apprehensive during the initial interview and seemed embarrassed about her problems. Her presenting complaint is that she has many worries. She is having difficulty reading, studying, and concentrating because she has become increasingly preoccupied with her worries over the past year. She spends hours each night rehashing the day's events, especially interactions with her professors and exchanges with other students in a particularly demanding course she is taking, but also some interactions with her parents, with whom she finds it difficult to get along. She says that she endlessly tries to correct in her mind any mistakes she might have made. She runs over every event, asking herself if she behaved properly or said the right thing. She does this at her desk when she is supposed to be reading, doing homework, or working on her undergraduate thesis, which interferes with her concentration. It is not unusual for her to look at the clock after such a period of rumination and find, to her surprise, that two or three hours have elapsed. She also does this at night, so she has not been sleeping well lately and finds herself unusually irritable and easily fatigued. She worries about a number of everyday, real-life problems and feels unable to stop her mind from worrying. For the past year, Mary has had fewer social contacts because of her nervous symptoms. Although she sometimes misses classes because of her symptoms, she continues to keep her grades at a B average. She tends to hide her symptoms from her family and her roommate and reports few problems with them. Recent medical and neurological examinations have ruled out substance use, general medical conditions, and neurological impairment as factors contributing to her problems.

* $\quad * \quad * \quad * \quad *$

What Axis I DSM-IV-TR diagnosis (or diagnoses) would you give to Mary, based on the information given in the paragraph above? 


\section{Of the following choices, how accurately do each of the following diagnostic categories represent Mary's primary Axis I presenting concern? (Circle one)}

\section{1.) Disorder of Infancy, Early \\ Childhood, or Adolescence}

2.) Substance-Related Disorder

3.) Schizophrenia or Other Psychotic Disorder

4.) Mood Disorder

5.) Anxiety Disorder

6.) Somatoform or Factitious Disorder

7.) Adjustment Disorder

8.) Other Condition that May be a Focus of Clinical Attention (VCode)

9.) No Diagnosis

10.) Other (please specify)

\begin{tabular}{|c|c|c|c|}
\hline 6 & 5 & 4 & 1 \\
\hline$\overline{V e r}$ & & Somewhat & $\overline{\mathrm{N}}$ \\
\hline Accurately & & Accurately & Accurately \\
\hline
\end{tabular}

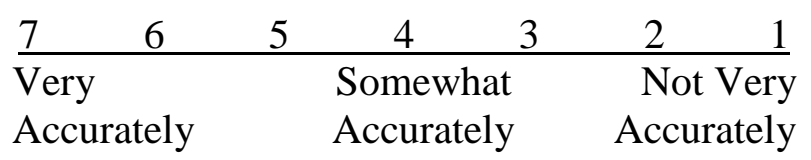

\begin{tabular}{|c|c|c|c|}
\hline 6 & 5 & 4 & 2 \\
\hline Jerv & & Somewhat & ery \\
\hline Accurately & & Accurately & Accurately \\
\hline
\end{tabular}

\begin{tabular}{|c|c|c|c|}
\hline 6 & 5 & 4 & 1 \\
\hline & & Somewhat & $\bar{N}$ \\
\hline Accurately & & Accurately & Accurately \\
\hline
\end{tabular}

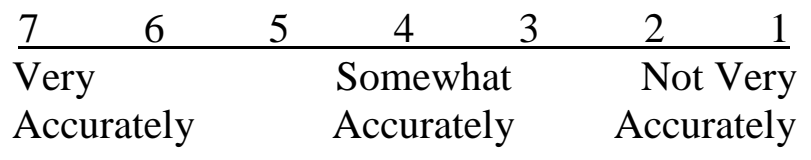

\begin{tabular}{|c|c|c|c|}
\hline 6 & 5 & 4 & 2 \\
\hline Jery & & Somewhat & ery \\
\hline Accurately & & Accurately & Accurately \\
\hline
\end{tabular}

\begin{tabular}{|c|c|c|c|}
\hline 6 & 5 & 4 & 1 \\
\hline Jer & & Somewhat & $\overline{\text { Tery }}$ \\
\hline Accurately & & Accurately & Accurately \\
\hline
\end{tabular}

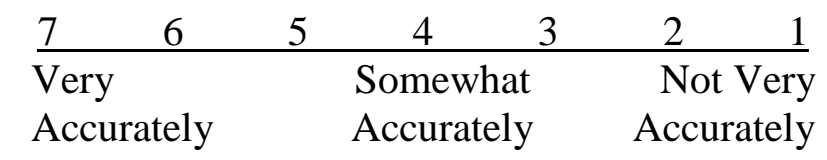

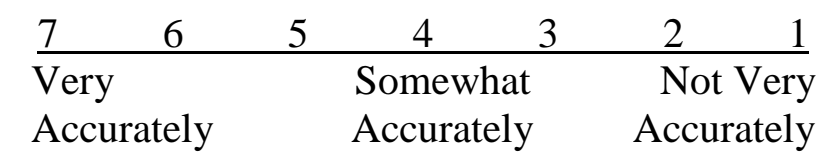

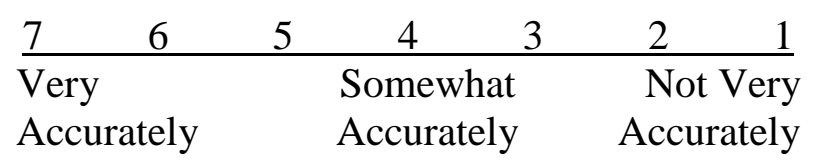




\section{The preceding clinical vignette of Mary was written to convey a client with an Anxiety Disorder. Please indicate how accurately this vignette depicts a person whose primary presenting concern is each of the following Anxiety Disorders. (Circle one)}

1.) How well does this vignette depict a person whose primary presenting concern is Panic Disorder (with or without Agoraphobia)?

2.) How well does this vignette depict a person whose primary presenting concern is Agoraphobia (without history of Panic Disorder)?

3.) How well does this vignette depict a person whose primary presenting concern is Obsessive-Compulsive Disorder?

4.) How well does this vignette depict a person whose primary presenting concern is Generalized Anxiety Disorder?

5.) How well does this vignette depict a person whose primary presenting concern is Posttraumatic Stress Disorder?

6.) How well does this vignette depict a person whose primary presenting concern is Social Phobia?

7.) How well does this vignette depict a person whose primary presenting concern is any other Anxiety Disorder not listed above (such as Adjustment Disorder with Anxiety, Anxiety Disorder Due to a General Medical Condition, SubstanceInduced Anxiety Disorder, Anxiety Disorder NOS, etc.)?

\begin{tabular}{|c|c|c|c|}
\hline 6 & 5 & 4 & 2 \\
\hline $\mathrm{Ve}$ & & Somewhat & Not Ver \\
\hline Accurately & & Accurately & Accurately \\
\hline
\end{tabular}

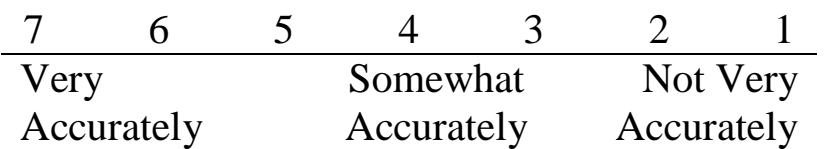

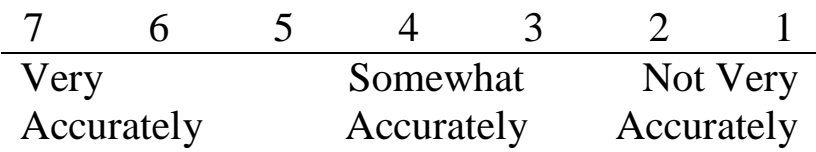

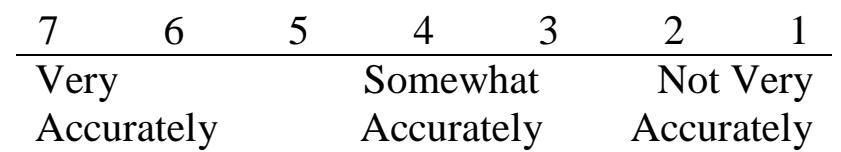

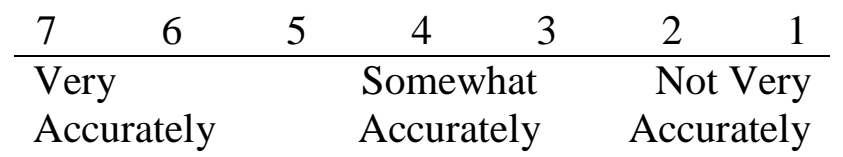

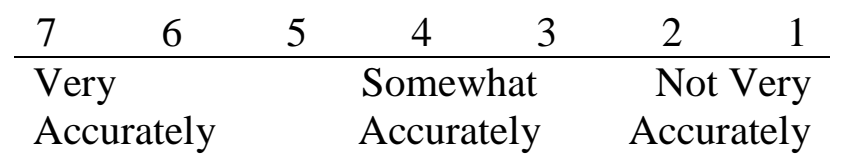

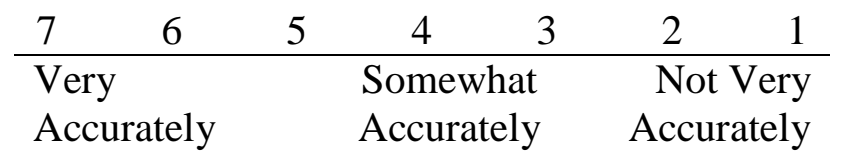
Accurately Accurately 


\section{Demographic Information}

1.) Age: years old

2.) Sex: (check one)

$\square$ Male $\quad \square$ Female

3.) Race/Ethnicity: (check one)

$\square$ Caucasian/White

African American/Black

$\square$ Asian American

$\square$ Hispanic American/Latino(a)

$\square$ Native American

Biracial/Multiracial*

International*

Other*

* Please specify:

4.) Highest degree obtained: (check one, then specify type - e.g., MSW, Clinical Psychology Psy.D., Counseling Psychology Ph.D., etc.)

$\square$ Bachelor's Degree:

$\square$ Masters Degree:

$\square$ Doctorate:

$\square$ Other:

5.) What year did you get your highest degree obtained?

6.) Since obtaining your degree, how many years have you been doing:

Diagnostic testing? years

Psychotherapy? years

7.) How many hours per week do you spend doing psychotherapy (i.e., individual, family, couples, or group therapy)? hours/week

8.) What are the settings in which you work? (Check all that apply. If you work in more than one setting, please rank the settings in order of time in which you spend in each, i.e., $1=$ primary setting, $2=$ secondary setting, etc.)

$\square \_$College/University setting
$\square \_$Elementary/Secondary school setting
$\square —$ Hospital
$\square$

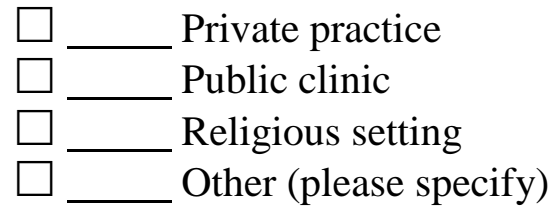




\title{
Appendix I:
}

\author{
Original 37 Vignette Versions
}

\section{Very High Religiousness}

Vignette 1: Mary considers herself to be a very devout Christian and describes herself as a person whose entire life revolves around Jesus. She states that her faith is more than just a belief system - it is a fundamental part of her individual identity, family life, and cultural upbringing. She spends much of her time doing church-related activities: she attends biweekly meetings for her church's finance committee, she attends church choir practice, and, on Sundays, she sings at two morning church services, teaches Sunday school, and goes to a smaller evening service. She has memorized hundreds of quotations from the Bible, and whenever she needs to make a decision about something, she thinks of the lessons she has learned from the many sermons she has heard and scriptures she has read, and then she asks herself, "What would Jesus do?" She gives liberally to youth ministry activities, foreign missions, local outreach ministries, and charitable organizations that her church sponsors. She freely shares her Christian faith with others and lives her life as a testimony of her faith in Christ.

Vignette 2: Mary considers herself to be a born-again Christian and describes herself as "truly devoted to Jesus." She views Jesus as the source of her strength and happiness, both directly and indirectly. The church services she attends typically last 3 or 4 hours and involve singing, giving testimony, and praising Jesus. Privately, she prays and reads the Bible several times a day, decorates her home with religious items, and talks to others about what she believes. She volunteers two or three times a month at a local homeless shelter and often gives what little money she has to her church to support its overseas missions and foreign ministries, seeing her community involvement as "my privilege as a true Christian woman." She feels strongly that "it's not enough for me just to sit in the pew - I need to be involved in meeting the needs others have of clothing, shelter, food, and friendship in order to truly serve the Lord."

Vignette 3: Mary considers herself to be an evangelical Christian and describes herself as a very religious person whose close personal relationship with Jesus Christ influences every area of her life. She believes in the inerrancy of the Bible and places her trust in its teachings, viewing it as the inspired word of God. She attends church 3-4 times each week, and, at a typical church service, she sings, praises God, and often publicly testifies how Jesus has touched her life whenever the spirit of the Lord moves her; she says she gets a great deal of comfort and affirmation out of the weekly sermon and leaves these services "filled with the love of Jesus." During the rest of the week, she often privately talks to Jesus to make sure her life is "on track." She regularly gives a sizeable percent of her income to the church, and, just last year, she donated an additional \$2000 towards the purchase of a new piano. She often talks to others about what she believes, and she makes it a point to tell others what an active role that Jesus has taken in her life since she was "born again." 


\section{$\underline{\text { High Religiousness }}$}

Vignette 4: Mary considers herself to be a devout Christian and describes herself as a very religious person with a close personal relationship with Jesus Christ. Religion is especially important to her because it answers many questions for her about the meaning of life. She attends church 3-4 times each week, and, at a typical church service, she enters into worship and praise. She memorizes verses from the Bible and prays to God several times each day. She faithfully gives a percentage of her income to the church. She believes that her religion is the one true faith and enthusiastically gives testimony of her faith in Jesus whenever the topic of her religion comes up in conversation.

Vignette 5: Mary considers herself to be a highly religious person and feels that her religious beliefs influence every area of her life. She describes her faith as a close personal relationship with Jesus Christ, referring to it as an internalized, heartfelt kind of belief that she has chosen because it feels right to her. She attends church 3-4 times each week, on Sunday mornings for worship services and at other times for choir practice, Bible study groups, and various church social events, where she gets a great deal out of the sermons her pastor delivers. When she is at home alone, she spends some of her time each day in prayer and Bible reading. Nearly every weekend, she volunteers at a local outreach ministry that her church sponsors. She believes that Jesus is the one true God and often talks about her beliefs with her friends, neighbors, and coworkers.

Vignette 6: Mary considers herself to be an evangelical Christian and thinks it is important to obey what the Bible says, believing its writings to be true and relevant to her life. She believes that the teachings in the Bible are divinely inspired truths to be taken seriously and obeyed. She attends church regularly, feeling that it is important for her to learn whatever she can from the sermons her pastor preaches. She spends periods of time in private prayer and meditation and makes sure that her behavior and speech reflect the teachings of her church. She occasionally donates money to charities that hold Biblical teachings similar to those of her own church and establish churches in underserved mission communities; she sometimes does charity work on the weekends as well, volunteering at a local homeless shelter. In this way, she lives her life as part of her testimony to her faith in Christ and freely witnesses her Christian faith with others whenever she feels it is appropriate.

Vignette 7: Mary considers herself to be an evangelical Christian and describes herself as a very religious person whose close personal relationship with Jesus Christ influences every area of her life. She believes that it is important for her to attend church regularly and places great emphasis on the sermons she hears there. She keeps well informed about her local church and regularly attends committee meetings to influence decisions that her church makes. At home, she prays to God several times a day in a respectful prayer attitude, and she often piously studies the Bible for an hour or two each day. She donates both time and money to several charitable organizations that her church sponsors. At work, she actively gives testimony of her faith in Christ with her coworkers, especially with those who do not regularly attend church, in an effort to get them to consider 
attending church or at least to tell them about the possibilities of an experience with Jesus.

\section{Moderate Religiousness}

Vignette 8: Mary considers herself to be a Christian and describes herself as a somewhat religious person who believes in God. She believes that the teachings in the Bible represent the truth and have practical value in the modern world; she interprets them as metaphors and parables to be interpreted and applied to her everyday life. She attends church services occasionally, and, when she does, she sings, prays, and learns something from the sermon, often feeling the presence of the Lord with her. She prefers to worship quietly and privately, by praying to God a few times a week and occasionally reading the Bible. She occasionally tithes a percentage of her income to her church. She refers to her beliefs as feeling right for her but says she can understand why other people might believe differently than she does.

Vignette 9: Mary considers herself to be somewhat religious and describes her religion as important to her. While she considers herself to be a Christian, she thinks it is more important to live a good life than to be a member of a particular church, though she also believes church attendance to be important. She attends church services about twice a month, where she participates actively and listens attentively to the sermon. She spends time trying to grow in understanding of her faith and often reads books and magazines about her faith. She sometimes does charity work on the weekends, volunteering at a local homeless shelter, to which she also donates used clothing, furniture, and other household items when she no longer needs them. She occasionally tells others about her beliefs if they seem receptive, but she does not "preach" to others, not wanting to offend them.

Vignette 10: Mary describes herself as somewhat religious, stating that she believes in God and has a personal relationship with Jesus. She refers to her faith as something she has always believed in because it is familiar and feels right to her. She enjoys spending time with others of her denomination, listens intently to sermons on Sundays, and frequently socializes with her church friends. She prays to God a few times each week and sometimes reads the Bible. She occasionally donates money to charities that preach Biblical teachings and establish churches in underserved mission communities. She believes everyone is entitled to their own religious beliefs and can live their lives as they choose, though she also feels that Christianity is the only religion that reveals God and is truly from God.

\section{$\underline{\text { Low Religiousness }}$}

Vignette 11: Mary considers herself to be a Christian and believes in Biblical teachings, but she also considers herself to be a skeptic who does not accept what she hears in regards to her religious beliefs without first questioning its validity. She describes attending church as something she occasionally does because it is "an important thing to 
do once in awhile," and because she believes it is important for her to learn occasionally from the lessons her pastor has to teach. She attends church about once a month. She prays to God sometimes, and she has read several passages from the Bible. Almost every year, around Christmas time, she volunteers at an annual soup kitchen which her church sponsors. She says that her beliefs seem to her to be true, but that she is not absolutely certain - "I could be wrong about what I believe."

Vignette 12: Mary considers herself to be a Christian but describes herself as not very religious - "I just believe in Christ and the Bible." She views herself as a moral and virtuous person, though she believes that she should attend church more often than she does. She attends church on Easter and around Christmas time and says she does so "to learn from the two most important sermons each year," and because she feels that she cannot be a Christian and never attend church. She has given considerable reflection to what she believes, and she occasionally has conversations with God whenever she feels the need to get a clearer perspective on her life. She occasionally volunteers at a local animal shelter and donates to Christian outreach ministries. She tells people she is Christian if they ask her and sometimes talks about religion with others, but she also believes everyone is entitled to their own religious beliefs and should live their lives as they choose.

Vignette 13: Mary describes religion as something she turns to in moments of despair. She says that she has experienced "the life-giving nature of the word of God," that she used to believe that church attendance was important, and that she used to place conviction in "the truths of my faith," but she is no longer certain of this conviction. She used to attend church occasionally but rarely finds the time to do so any more. She has read most of the Bible and thought it sounded true, but she found it to be somewhat dry and boring. At work, she gives to the Salvation Army or other legitimate charities at least once each year. She is most familiar with Christian teachings but says she is open-minded towards the teachings of all faiths.

\section{Very Low Religiousness}

Vignette 14: Mary considers herself to be a "nonreligious" person. She believes in God and the revelations of the Bible, but she feels that living a moral life is more important than attending a church. She says that the only times she attended church in her life were for a wedding and two funerals. She occasionally reads about religion, but she says she has never understood why religion is so important to so many people. She feels that it is important and right to live a moral life, to help others in need, and to give to charities, even if only occasionally. She believes in open dialogue between individuals regardless of their religious or denominational affiliation, but ever since she was a teenager, Mary has been suspicious of people who preach to others or tell others what they should believe.

Vignette 15: Mary used to be a Christian but no longer considers herself to be a religious person. She believes in God, the Bible, and following the golden rule. She used to attend 
church occasionally, but she has not done so in ten years. She also used to pray every day, and on rare occasions she still prays privately, but she does so very infrequently. At work, she gives the minimum pledge allowed by her company to the United Way. She believes everyone is entitled to their own religious beliefs and can live their lives as they choose.

\section{$\underline{\text { Very High Spirituality }}$}

Vignette 16: Mary considers herself to be very spiritual and describes herself as a person whose entire life revolves around her relationship with the universe. She has always felt a sense of trust and security because she "knows without a shadow of a doubt" that "the universe provides." She spends much of her time doing spiritual activities: she attends a personal growth group on spiritual issues, she attends a weekly meditation class, and twice a week she attends a spirituality class at her local university. She has memorized hundreds of quotations from various spiritual and religious texts and is assembling them into a comparative anthology of world scripture. She gives liberally to youth development programs, urban outreaches, community improvement projects, and charitable organizations. She freely shares her spiritual beliefs with others and lives her life as a testimony of her faith in the essential beauty and order of the universe.

Vignette 17: Mary considers herself to be a spiritual seeker and describes herself as "truly devoted to the divine." She describes her faith as a close personal relationship among herself, the divine, like-minded others, and the universe itself, referring to it as an internalized, heartfelt kind of belief that she chose because "it just makes sense." She attends weekly meditation services that typically last for 3 or 4 hours and involve mantra, visualization, and clearing the mind of worldly concerns. Privately, she prays and reads spiritual or religious texts from diverse traditions several times a day, decorates her home with items from several different faiths, and talks to others about what she believes. She volunteers two or three times a month at a local homeless shelter and often gives what little money she has to girls and boys club programs to support their youth development projects, seeing her community involvement as "my privilege as a truly spiritual woman." She feels that "it's not enough for me just to sit around and read about this stuff - I need to be involved in order to truly serve the universe."

Vignette 18: Mary considers herself to be a "mystical seeker" and describes herself as a very spiritual person whose close personal relationship with the universe influences every area of her life. She states that her faith is more than just a belief system - it is a fundamental part of her individual identity, family life, and cultural upbringing. She typically worships by attending mantra workshops - chant rituals that last for half a day or more - noting that at times this experience is incredibly powerful for her. During the rest of the week, she often privately talks to a higher power to make sure her life is on track. She regularly gives a generous percentage of her income to local charities, and just last year she donated an additional \$2000 towards the purchase of a new stove for a nearby homeless shelter. She often talks to others about what she believes, and makes it a 
point to tell others what an active role that her personal relationship with the universe has taken in guiding her life since she "realized the truth of the universe."

Vignette 19: Mary considers herself to be a seeker and describes herself as a very spiritual person whose soul is intimately connected with other people, the divine, and the universe itself. She describes her faith as an intuitive, intensely personal connection with the universe in which she, other people, the divine, and the fabric of the universe itself are all part of the same divine unity. She typically worships by participating in spiritual workshops at a local university, or by attending formal meditation classes, until her soul reaches a state of bliss or peaceful rest. She reads the writings of the world's major religions nearly every day, and at least once a day she says prayers to the divine from the world's major religions. She frequently volunteers for the American Red Cross and helps them organize fundraisers and local community outreach activities. She calls her worldview "one true way of looking at the universe," and she often enthusiastically invites people she knows to participate in the spiritual workshops she attends, feeling that everyone should have the opportunity to "get in touch with themselves and the divine as I have."

\section{$\underline{\text { High Spirituality }}$}

Vignette 20: Mary considers herself to be a very spiritual person with a close personal relationship with the universe. She has always felt a sense of trust and security because she is certain that fate will show her the right way to live her life. She typically practices with others by attending chant rituals, guided meditations, and spiritual healing services. She memorizes verses from various spiritual and religious texts and prays to the divine several times each day. She faithfully gives a percentage of her income to charities. In this way, she lives her life as a testimony of her faith in the essential beauty and order of the universe and freely shares her spiritual beliefs with others whenever she feels it is appropriate.

Vignette 21: Mary considers herself to be a "mystical seeker" and feels that her relationship with the divine and with the environment influences every area of her life. Her spirituality is especially important to her because it gives her a general sense about the meaning of life. She attends weekly spiritual healing services and has not missed a meeting in the past 5 years. She spends periods of time in private thought and meditation and makes sure that her behavior reflects the core teachings that the major world faiths have in common. Nearly every weekend, she volunteers at a local homeless shelter or an environmental preservation society. She often talks about her beliefs with her friends, neighbors, and coworkers, and, whenever she feels it is appropriate, she gives testimony of her experiences with meditation in an effort to get others to consider meditating themselves.

Vignette 22: Mary considers herself to be a "spiritual seeker" and thinks it is important to "seek the underlying will or plan of the divine." She describes her faith as a close personal relationship with the divine and with the universe itself. She attends a spiritual 
healing service at least 2 or 3 times each week. She prays to the divine several times a day and usually meditates for an hour or two each day. She occasionally donates money to Care, the American Red Cross, and other secular hunger relief charities, and she sometimes does charity work on the weekends as well, volunteering at a local homeless shelter. She takes great joy in talking with others about what they believe, and she frequently shares her beliefs about spirituality with her friends.

Vignette 23: Mary considers herself to be "a seeker" and describes herself as a very spiritual person whose close personal relationship with the divine influences every area of her life. She describes her faith as an intensely close and personal relationship among herself, the divine, like-minded others, and the universe itself. She typically worships 3-4 times each week by attending meditation classes, which she finds spiritual and uplifting. She meditates several times a day, and she usually reads books about religion or spirituality nearly every day. She donates both time and money to several charitable organizations that her prayer partners from her spiritual healing group sponsor. She takes great joy in talking with others about what she believes if they seem open-minded and receptive, but she says that she never preaches to others or imposes her beliefs on them unless they bring up the topic first.

Vignette 24: Mary considers herself to be "a mystical seeker" who is "in touch with the universe," and she describes herself as a very spiritual person whose close personal relationship with the divine influences every area of her life. She describes her faith as an intensely close and personal relationship with the divine and with the universe itself, referring to it as an internalized, heartfelt kind of belief that she believes in because it feels right to her. She typically worships by meditating quietly on her own, or occasionally by attending guided meditations with other people who regard spirituality as important. She meditates at least 3-4 times a week, and prays to a higher power each morning and night. She regularly volunteers for the American Red Cross and helps them organize fundraisers and local community outreach activities in her neighborhood. She often invites people she knows to join the spiritual healing services she attends, feeling that everyone should have the opportunity to "get in touch with themselves and the universe as I have."

Vignette 25: Mary considers herself to be a "very spiritual" person with "a close personal relationship with the divine." She believes that events happen for a reason and trusts that a divine power is guiding her life. She typically worships every day by lighting a candle and saying a prayer to the divine. When she is at home alone, she spends some of her time talking with the divine to make sure her life is on track. She often volunteers her time at a local homeless shelter, to which she also donates clothing, furniture, and other household items when she no longer needs them. She enthusiastically gives testimony of her faith in the divine whenever the topic of spirituality comes up in conversation. 


\section{$\underline{\text { Moderate Spirituality }}$}

Vignette 26: Mary considers herself to be a seeker and describes herself as a somewhat spiritual person who believes in the divine. She feels a sense of trust and security because she has a general feeling that "the universe provides." She attends spiritual healing services occasionally, and, when she does, she often feels the presence of the divine with her. She prefers to worship quietly and privately, by praying to a divine power a few times a week and occasionally reading books about religion or spirituality. She faithfully gives a percentage of each of her paychecks to the United Way. She refers to her beliefs as feeling right for her but says she can understand why other people might believe differently than she does.

Vignette 27: Mary considers herself to be somewhat spiritual and describes her faith as important to her. She feels a sense of trust and security because she is hopeful that the universe will show her the right way to live her life. She attends a personal growth group on spiritual issues about twice a month, where she sits quietly and listens attentively to the personal spiritual experiences that others share. She spends time trying to grow in understanding of the universe and often reads books and magazines about spirituality. She sometimes does charity work on the weekends, volunteering at a local homeless shelter, to which she also donates clothing, furniture, and other household items when she no longer needs them. She occasionally tells others about her spiritual beliefs if they seem receptive, but she does not "preach" to others, not wanting to offend them.

Vignette 28: Mary describes herself as somewhat spiritual, stating that she believes in a higher power that gives the universe meaning and purpose, though beyond that she is not sure exactly what she believes. She describes her faith as a commitment to a personal relationship among herself, a higher power, like-minded others, and the universe itself. She meditates regularly, enjoys spending time with other people who meditate, and frequently socializes with friends from her Yoga class. She prays to a higher power a few times each week and sometimes reads about meditation. She occasionally donates money to Care, the American Red Cross, and other secular hunger relief charities. She believes everyone is entitled to their own beliefs and can live their lives as they choose, though she also feels that her meditative worldview makes the most sense to her out of all the different belief systems she has encountered.

Vignette 29: Mary describes herself as a somewhat spiritual person who considers herself to be an open-minded seeker. She describes her faith as "a kind of relationship I have with the universe," and she refers to her faith as something she believes in because it feels right to her. On occasion, she attends chant rituals, guided meditations, or spiritual healing services, out of curiosity to hear about the spiritual experiences of others. She prays to a divine being a few times each week, and she privately meditates about twice a month. Sometimes, she volunteers for the American Red Cross whenever they organize fundraisers and local community outreach activities in her neighborhood. She occasionally invites others to come to the spiritual healing services she attends if they ask 
her about what she believes, but she only does this if they seem interested in spiritual matters and bring up the topic first.

Vignette 30: Mary considers herself to be a spiritual seeker who is curious about how the universe works. She describes her faith as a close relationship with the universe and a connection to spiritual traditions associated with traditional American life [or, the traditional African American experience], and she refers to her faith as something she "just came to believe," though she is unsure of exactly how she came to believe this way. She attends a personal growth group on spiritual issues occasionally, and about once a month she attends a meditation and Yoga class. She prays to a higher power every once in awhile and privately meditates two or three times a year. She often volunteers her time at a local homeless shelter. She occasionally tells people about the benefits of meditation and yoga if the topic comes up.

\section{$\underline{\text { Low Spirituality }}$}

Vignette 31: Mary considers herself to be "not very spiritual - I just believe in some kind of a higher power." She describes her faith as something which used to be important to her but which no longer has much relevance to her life any more. She attends meditation classes 2 or 3 times each month. She prays to a higher power sometimes, and she has read passages from several religious texts. She volunteers at a local homeless shelter around Thanksgiving almost every year. She tells people about her spiritual beliefs if they ask her, but she also believes everyone is entitled to their own beliefs and should live their lives as they choose.

Vignette 32: Mary considers herself to be a person of faith, but she does not accept what she hears in regards to her beliefs without first questioning its validity. She views herself as "a little bit spiritual" and believes in a divine being, but she also believes that it is not necessary for her to act in certain ways or follow prescribed rituals in order to be a good or spiritual person. She used to attend a biweekly meditation class, but she has not found time to attend for more than a year. She has given considerable reflection to what she believes, and she occasionally has "conversations with the universe" whenever she feels the need to get a clearer perspective on her life. She occasionally volunteers at a local animal shelter and donates to nature conservation and preservation organizations. She says that her spiritual beliefs seem to be true, but that she is not absolutely certain - "I could be wrong about what I believe."

Vignette 33: Mary describes her faith as something she turns to in moments of despair. She has a general conviction that "the universe provides," though she says that this is the only thing she is convinced is true. She used to attend spiritual healing services with some like-minded prayer partners occasionally, but she rarely finds the time to do so any more. She meditates on the meaning of life occasionally, and she prays to a divine being every once in awhile. At work, she gives to the United Way at least once each year. She is open-minded towards the teachings of all faiths, but she says that she has not yet found a faith which she feels is worthy of holding a central place in her life. 
Vignette 34: Mary considers herself to be open-minded, curious about the way the universe works, and "a little bit spiritual." She has felt moments of deep meaning in her life and connection to a higher power who has shown her the right way to live her life, though she states that the last time she experienced such a moment was ten years ago. Three or four times each year, she attends a meditation and Yoga workshop. She prays to a higher power sometimes, and she occasionally reflects on the meaning of life and her place in the universe. She donates to charity at least once or twice each year. She sometimes tells people that she believes in a higher power if the topic comes up, but she usually does not share her personal beliefs with other people.

\section{Very Low Spirituality}

Vignette 35: Mary considers herself to be a "non-spiritual" person. She believes in following the golden rule, and she feels that living a moral life is more important than worshipping a higher power. She says that the only times she attended "spiritual events" in her life were for a wedding, two funerals, and a Yoga class. She occasionally reads about spirituality, but she says she has never understood why religion is so important to so many people. She feels that it is good to live a moral life, to help others in need, and to give to charities occasionally. She believes in open dialogue between individuals, but ever since she was a teenager, Mary has been suspicious of people who preach to others or tell others what they should believe.

Vignette 36: Mary used to be a spiritual person but no longer considers herself to be spiritual. She believes that it is not necessary for her to feel spiritual in order to live her life and to be happy. She used to attend spiritual healing services occasionally, but she has not done so in ten years. She also used to meditate, and, on rare occasions, she still prays to the divine privately, but she does so very infrequently, and she has not meditated in at least ten years. At work, she gives the minimum pledge allowed by her company to the United Way. She believes everyone is entitled to their own spiritual beliefs and can live their lives as they choose.

\section{New Baptist Vignette: High Religiousness}

Vignette 37: Mary considers herself to be a devout Baptist woman and describes herself as a person whose entire life revolves around her church. She views her congregation and the relationship she has with Christ as the source of her strength and happiness. She attends church at least twice a week and enjoys singing, praising God, learning from her pastor's weekly messages, and enjoying fellowship with her Christian friends. Privately, she places great emphasis on the sermons she hears, reflecting on their meaning and trying her best to apply them to her Christian life. She frequently donates money to charities that preach Biblical teachings following the Baptist traditions and establish churches in underserved mission communities; she faithfully tithes a percentage of her income to her church. In this way, she lives her life as a testimony of her faith in Christ and freely shares her Christian faith with others whenever she feels it is appropriate. 


\section{Appendix J:}

Pilot Study 2 Questionnaire

\section{$\underline{\text { Religious/Spiritual Expert Raters Questionnaire }}$}

On the following pages are a series of 37 vignettes that depict a series of women (all of them named Mary) who have varying levels of religiousness or spirituality. They have been written to vary systematically from depictions of very high to very low religiousness, or from very high to very low spirituality. As these vignettes were being developed, the following operational definitions of religiousness and spirituality were used:

Religiousness is a search for the sacred (or non-sacred things such as social contact or appearing virtuous to your neighbors) in a traditional institutional context that typically facilitates searches for the sacred.

Spirituality is a personal search for the sacred which may or may not occur in a traditional institutional context that typically facilitates searches for the sacred.

The sacred is an ultimate, transcendent, or divine being, reality, or truth as perceived by an individual.

(Taken from Hill, Pargament, Hood, McCullough, Swyers, Larson, \& Zinnbauer's, 2000, definitional criteria, which were based on a 1997 conference series in which 60 psychologists, medical doctors, neuroscientists, and substance abuse counselors attempted to define the terms religiosity and spirituality)

Please read each vignette, keeping in mind the above definitions of religiousness and spirituality as well as your own personal and professional views of what religiousness and spirituality mean. The next step is to then indicate on the scales provided the extent to which each vignette represents an authentic expression of religiousness and spirituality for both Black and White persons. 


\section{Vignette 1}

Mary considers herself to be a very devout Christian and describes herself as a person whose entire life revolves around Jesus. She states that her faith is more than just a code of behavior - it is a fundamental part of her individual identity, family life, and cultural upbringing. She spends much of her time doing church-related activities; she attends biweekly meetings for her church's finance committee, she attends church choir practice, and on Sundays she sings at three morning church services, attends Sunday school, and goes to a smaller evening service. She has memorized hundreds of quotations from the Bible, and whenever she needs to make a decision about something, she thinks of the lessons she has learned from the many sermons she has heard, and then she asks herself, "What would Jesus do?" She gives liberally to youth ministry activities, foreign missions, local outreach ministries, and charitable organizations that her church sponsors. She freely shares her Christian faith with others and lives her life as a testimony of her faith in Christ.

1.) How well does this vignette depict a Religious person whose religious faith is Christian and whose church denomination is Baptist? [7 = Very Accurately

(Please Select One)

6

5

[Response options in drop-down box were:]

2.) How well does this vignette depict a Spiritual person who is not religious?

(Please Select One)

3.) How well does this vignette represent a Baptist expression of religious faith for a White person?

(Please Select One)

4.) How well does this vignette represent a Baptist expression of religious faith for a Black person?

(Please Select One)

5.) If you rated this vignette as low in accuracy (i.e., 1-4) for question 1 above, what changes would you make to this vignette so that it more accurately represents a Religious person (Black or White) who is both Christian and a Baptist? 


\section{Vignette 2}

Mary considers herself to be a born-again Christian and describes herself as "truly devoted to Jesus." She views Jesus as the source of her strength and happiness, both directly and indirectly. The church services she attends typically last 3 or 4 hours and involve singing, giving testimony, and praising Jesus. Privately, she prays and reads the Bible several times a day, decorates her home with religious items, and talks to others about what she believes. She volunteers two or three times a month at a local homeless shelter and often gives what little money she has to her church to support its overseas missions and foreign ministries, seeing her community involvement as "my privilege as a true Christian woman." She feels strongly that "it's not enough for me just to sit in the pew - I need to be involved in order to truly serve the Lord."

1.) How well does this vignette depict a Religious person whose religious faith is Christian and whose church denomination is Baptist?

(Please Select One)

2.) How well does this vignette depict a Spiritual person who is not religious?

(Please Select One)

3.) How well does this vignette represent a Baptist expression of religious faith for a White person?

(Please Select One)

4.) How well does this vignette represent a Baptist expression of religious faith for a Black person?

(Please Select One)

5.) If you rated this vignette as low in accuracy (i.e., 1-4) for question 1 above, what changes would you make to this vignette so that it more accurately represents a Religious person (Black or White) who is both Christian and a Baptist? 
[Vignettes 3-15 appeared here] 


\section{Vignette 16}

Mary considers herself to be very spiritual and describes herself as a person whose entire life revolves around her relationship with the universe. She has always felt a sense of trust and security because she "knows without a shadow of a doubt" that "the universe provides." She spends much of her time doing spiritual activities; she attends a personal growth group on spiritual issues, she attends a weekly meditation class, and at least three times a week she does Yoga at the gym. She has memorized hundreds of quotations from various spiritual and religious texts and is assembling them into a comparative anthology of world scripture. She gives liberally to youth development programs, urban outreaches, community improvement projects, and charitable organizations. She freely shares her spiritual beliefs with others and lives her life as a testimony of her faith in the essential beauty and order of the universe.

1.) How well does this vignette depict a Spiritual person whose personal faith is not affiliated with any religious denomination?

(Please Select One)

2.) How well does this vignette depict a Religious person who is Baptist?

(Please Select One)

3.) How well does this vignette represent spirituality for a White person who is not affiliated with any religious denomination?

(Please Select One)

4.) How well does this vignette represent spirituality for a Black person who is not affiliated with any religious denomination?

(Please Select One)

5.) If you rated this vignette as low in accuracy (i.e., 1-4) for question 1 above, what changes would you make to this vignette so that it more accurately represents a Spiritual person (Black or White) whose personal faith is not affiliated with any religious denomination? 


\section{Vignette 17}

Mary considers herself to be a spiritual seeker and describes herself as "truly devoted to a higher power." She describes her faith as a close personal relationship with herself, with a higher power, with like-minded others, and with the universe itself, referring to it as an internalized, heartfelt kind of belief that she chooses to believe in because "it just makes sense." She attends weekly meditation services that typically last for 3 or 4 hours and involve mantra, visualization, and clearing the mind of worldly concerns. Privately, she prays and reads spiritual or religious texts several times a day, decorates her home with items from several different faiths, and talks to others about what she believes. She volunteers two or three times a month at a local homeless shelter and often gives what little money she has to girls and boys club programs to support their youth development projects, seeing her community involvement as "my privilege as a truly spiritual woman." She feels that "it's not enough for me just to sit around and read about this stuff - I need to be involved in order to truly serve the universe."

1.) How well does this vignette depict a Spiritual person whose personal faith is not affiliated with any religious denomination?

(Please Select One)

2.) How well does this vignette depict a Religious person who is Baptist?

(Please Select One)

3.) How well does this vignette represent spirituality for a White person who is not affiliated with any religious denomination?

(Please Select One)

4.) How well does this vignette represent spirituality for a Black person who is not affiliated with any religious denomination?

(Please Select One)

5.) If you rated this vignette as low in accuracy (i.e., 1-4) for question 1 above, what changes would you make to this vignette so that it more accurately represents a Spiritual person (Black or White) whose personal faith is not affiliated with any religious denomination? 
[Vignettes 18-36 appeared here] 


\section{Vignette 37}

Mary considers herself to be a devout Baptist woman and describes herself as a person whose entire life revolves around her church. She views her congregation and the relationship she has with Christ as the source of her strength and happiness. She attends church at least twice a week and enjoys singing, praising God, learning from her pastor's weekly messages, and taking fellowship with her Christian friends. Privately, she places great emphasis on the sermons she hears, reflecting on their meaning and trying her best to apply them to her Christian life. She frequently donates money to charities that preach Biblical teachings and establish churches in underserved mission communities, and she faithfully tithes a percentage of her income to her church. In this way, she lives her life as a testimony of her faith in Christ and freely shares her Christian faith with others whenever she feels it is appropriate.

1.) How well does this vignette depict a Religious person whose religious faith is Christian and whose church denomination is Baptist?

(Please Select One)

2.) How well does this vignette depict a Spiritual person who is not religious?

(Please Select One)

3.) How well does this vignette represent a Baptist expression of religious faith for a White person?

(Please Select One)

4.) How well does this vignette represent a Baptist expression of religious faith for a Black person?

(Please Select One)

5.) If you rated this vignette as low in accuracy (i.e., 1-4) for question 1 above, what changes would you make to this vignette so that it more accurately represents a Religious person (Black or White) who is both Christian and a Baptist? 


\section{Demographic Information}

1.) Age: $\quad$ years old

2.) Sex: (check one)

$\square$ Male $\quad \square$ Female $\quad \square$ Other (specify: )

3.) Race/Ethnicity: (check one)

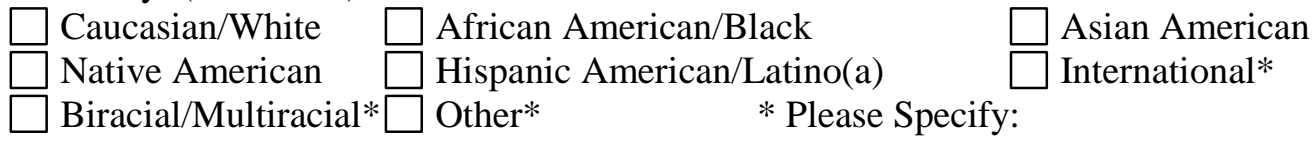

4.) Degrees obtained: (check all that apply, then specify type - e.g., MDIV, Religious Studies Ph.D., B.A. in Church History, etc.)
$\square$ Associate's Degree:
$\square$ Bachelor's Degree:
Masters Degree:
Doctorate:
$\square$ Other:

5.) What year did you get your highest degree obtained?

6.) What are the settings in which you work? Please check all that apply. If you work in more than one setting, please rank the settings in order of time in which you spend in each, i.e., primary setting, secondary setting, etc. Also, for each setting in which you work, please specify the name of the setting next to it.

\begin{tabular}{ll}
\multicolumn{1}{c}{ Setting } & Name \\
\hline$\square$ N/A Church & \\
N/A Temple & N/A School \\
N/A Hospital \\
N/A Pastoral Counseling \\
N/A Mission \\
N/A Public clinic \\
N/A Other secular setting \\
$\square$ N/A Other religious setting \\
$\quad{ }^{+}$[Response options included N/A, Primary, Secondary, Tertiary]
\end{tabular}

7.) As a religious/spiritual professional, what are your main professional responsibilities? Please choose at least one and up to five responsibilities, and briefly list them below.

8.) Please indicate how many years you have been doing each one of the professional responsibilities you listed below.

9.) Please also indicate approximately how many hours per week you typically spend doing each of the professional responsibilities you listed below.

\begin{tabular}{lccc} 
& $\begin{array}{c}\text { 7.) Briefly List the } \\
\text { Responsibility }\end{array}$ & $\begin{array}{c}\text { 8.) Years } \\
\text { Spent } \\
\text { Doing This }\end{array}$ & $\begin{array}{l}\text { 9.) Hours/Week } \\
\text { Spent Doing } \\
\text { This }\end{array}$ \\
\hline Responsibility 1: & years & hours/week \\
Responsibility 2: & years & hours/week \\
Responsibility 3: & years & hours/week \\
Responsibility 4: & years & hours/week \\
Responsibility 5: & years & hours/week
\end{tabular}




\title{
Appendix K:
}

\section{Pilot Study 3 Questionnaires (Magnitude Estimation Scaling Pilot Study)}

\author{
Introductory Letter: Magnitude Estimation Scaling Pilot Study \\ Clinical Judgment Faith Bias: The Impact of Faith and Multicultural Competence on Clinical \\ Judgment
}

The purpose of this project is to examine clinical judgment faith bias, a hypothesized tendency for some clinicians to make more negative clinical judgments for highly religious or highly spiritual clients than for otherwise identical moderately religious, moderately spiritual, nonreligious, or nonspiritual clients. To investigate this hypothesis, the researchers will be giving psychologists vignettes describing clients who have low, moderate, or high levels of religiousness or spirituality but are otherwise identical and testing to see whether psychologists respond differently to different vignettes. Before we do this, though, we need to select vignettes that adequately represent low, moderate, and high levels of religiousness and spirituality. Right now, we have 28 vignettes. We need to select the 6 that best represent low, moderate, and high religiousness and spirituality.

For this project, you will complete a magnitude estimation scaling task, which will allow us to select the 6 best vignettes for our purposes. The technique of magnitude estimation scaling originated from research on sensory psychophysics scaling. In the social sciences, magnitude estimation scaling is an alternative to Likert-type and other categorical scales.

Because the act of assigning numbers to the perceived strength of social stimuli is unfamiliar, two exercises are presented first. Your ratings from this initial exercise also serve as a necessary correction to regression bias in the magnitude estimation scaling procedure that follows.

You will be asked to do five things in this study:

1.) You will be asked to fill out a brief demographic questionnaire.

2.) You will be asked to assign number estimates to the length of 12 lines in comparison to a $50 \mathrm{~mm}$. reference line.

3.) You will be given 12 numbers and asked to draw lines to match the length of those numbers in comparison to a $50 \mathrm{~mm}$. reference line.

4.) You will be given a reference vignette describing a moderately religious or spiritual client, which will be paired with an assigned number of 50 . You will then be given a pool of 14 vignettes and asked to assign a number estimate to each vignette representing how religious or spiritual each vignette is.

5.) You will be given that same reference vignette again describing a moderately religious or spiritual client, which will be paired with a $50 \mathrm{~mm}$ reference line this time. You will then be given the same pool of 14 vignettes again and asked to draw a line for each vignette representing how religious or spiritual each vignette is.

It is anticipated that it will take you approximately 30 to 60 minutes to complete this study.

The purpose of having you do this is so that we may mathematically estimate the magnitude of the religiousness or spirituality of each vignette, so that we may select six vignettes. Two vignettes will be selected representing moderate religiousness and moderate spirituality, and four more vignettes will be selected representing approximately half and twice the degree of religiousness and spirituality as the moderate vignettes represent. These vignettes will represent low, moderate, and high levels of religiousness and spirituality and will enable the degree of client faith - both religiousness and spirituality - to be treated as continuous variables.

All of your information will be anonymous. You will only be asked demographic information questions; no identifying information will be collected, and your demographic information will be kept separate from the number estimates and lines you will draw. We will have no way of identifying who you are or even connecting your number estimates and line drawings with your demographic information (as long as you don't write your name on any of the sheets). 
The foreseeable risks or ill effects from participating in this study are minimal. You will be asked to draw lines and estimate the length of other lines, which may be a little boring, but this is not anticipated to make you feel uncomfortable. If you do develop uncomfortable feelings during your participation in this research project, counseling services are available to you through the Counseling Center at Ball State University (765-285-1736). You will be responsible for the costs of any care that is provided. [Note: Ball State students and faculty and staff may have some or all of these services provided to them at no cost.] It is understood that in the unlikely event of an injury or illness of any kind as a result of your participation in this research project that Ball State University, its agents and employees will assume whatever responsibility is required by law. If any injury or illness occurs in the course of your participation in this research project, please notify the principal investigator.

You may gain some direct benefits from your participation in this study. If you are a counseling psychology or psychological sciences student, you may receive research participation credit in exchange for the time you spend doing this study. Indirectly, you will also be making an important contribution to scientific learning. This study will contribute to the existing psychology of religion research literature because it will result in the generation of clinical vignettes that have been mathematically generated to represent continuous interval data - the medium religiousness (or spirituality) vignettes will represent approximately twice the religiousness (or spirituality) as the low religiousness (or spirituality) vignettes, and the high religiousness (or spirituality) vignettes will represent approximately twice the religiousness (or spirituality) as the medium religiousness (or spirituality) vignettes. The other vignettes in the research literature were not developed in such a rigorous way, so this will allow us to draw conclusions that could not otherwise be drawn using existing vignettes. Thus your participation is important - only through the time and effort of students like you can the researchers in this study make this contribution. So we thank you very much for your willingness to help.

Your participation in this study is completely voluntary and you are free to withdraw from the study at any time for any reason without penalty or prejudice from the investigators. If you withdraw from the study, your data will not be used, and your materials will be destroyed. However, if you are participating for research participation credit, you will still receive credit for the amount of time that you have spent participating. Please feel free to ask any questions of the investigators at any time during the study.

For your rights as a research subject, the following person may be contacted: Coordinator of Research Compliance, Office of Academic Research and Sponsored Programs, Ball State University, Muncie, IN 47306, (765) 285-5070. You may take this letter with you to keep for future reference.

Thank you for your time.

Sincerely,

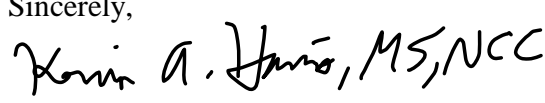

Kevin A. Harris, MS, NCC

Doctoral Candidate in Counseling Psychology

Ball State University

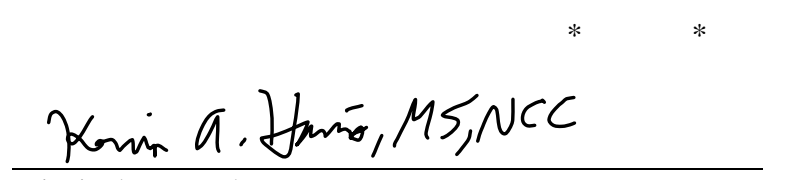

Principal Researcher

Kevin A. Harris, MS, NCC

Department of Counseling Psychology and Guidance Services

Ball State University

Muncie, IN 47306

Telephone: (305) 558-0151 Ext. 2261

E-mail: kaharris@bsu.edu

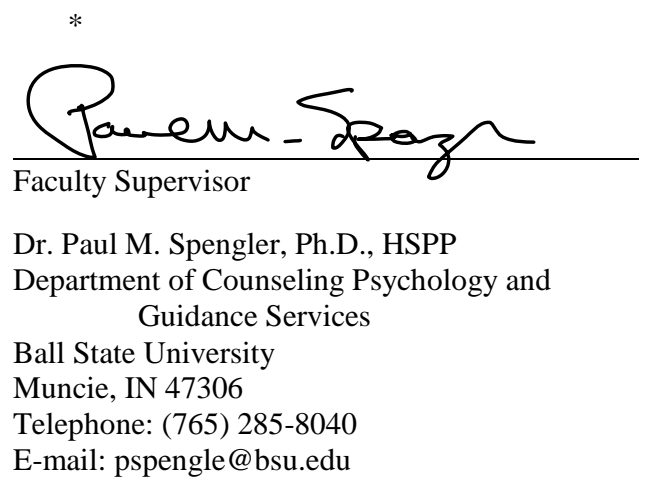

Edition Date: 12/08/07 
Participant R 01

\section{Clinical Judgment Faith Bias: \\ The Impact of Faith and Multicultural \\ Competence on Clinical Judgment \\ Pilot Study: Magnitude Estimation Scaling}

\section{Booklet 1: \\ Number Estimates}

[Given to both Groups of College Students] 
Participant R 01

\section{Magnitude Estimation Scaling: Number Estimates}

This booklet contains a series of line lengths. Please leaf through the next couple pages of this booklet and note that some of the lines are longer than the first line and some are shorter. Your task is to tell how much longer or shorter each line is compared to the first line. The first line is your reference. It has been given the number 50 .

\section{[50 ]}

The number 50 is your reference. All you need to do is write a number in the box for each line. The longer a line appears to be compared to the reference line, the bigger the number you will write in compared to 50. For example, if one of the lines seems about two times longer than the reference line, you would write in the number 100. If a line were ten times longer, you would write in the number 500. On the other hand, some of the lines are shorter than the reference line. If a line were about one half as long, you would write in a number about one half of 50 , about 25 . Another line about one tenth as long would be given the number one tenth of 50,5. Give each line a number, whatever seems appropriate, to express how the line compares to the reference line: The longer the line, the bigger your number compared to 50 . The shorter the line, the smaller your number compared to 50 . No rulers, please. I am only interested in your general impressions. 
Participant R 01
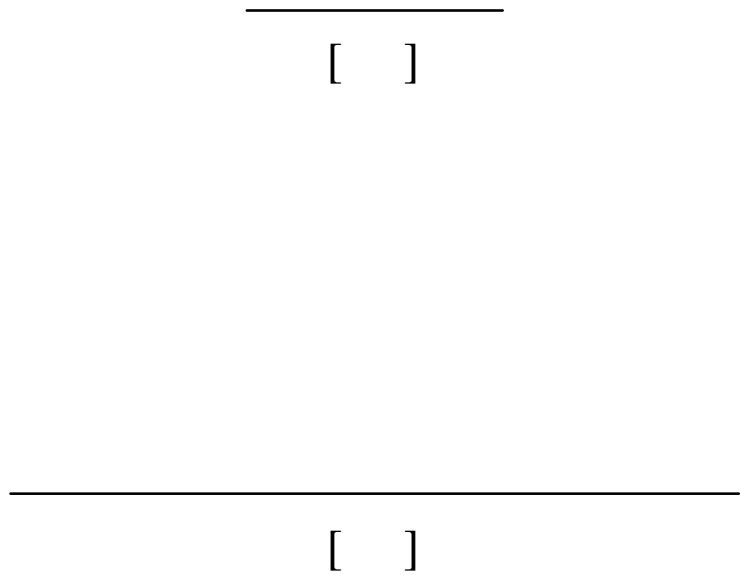

[ ]

[ ] 
Participant R 01

[ ]

[ ]

[ ]

[ ] 
Participant R 01

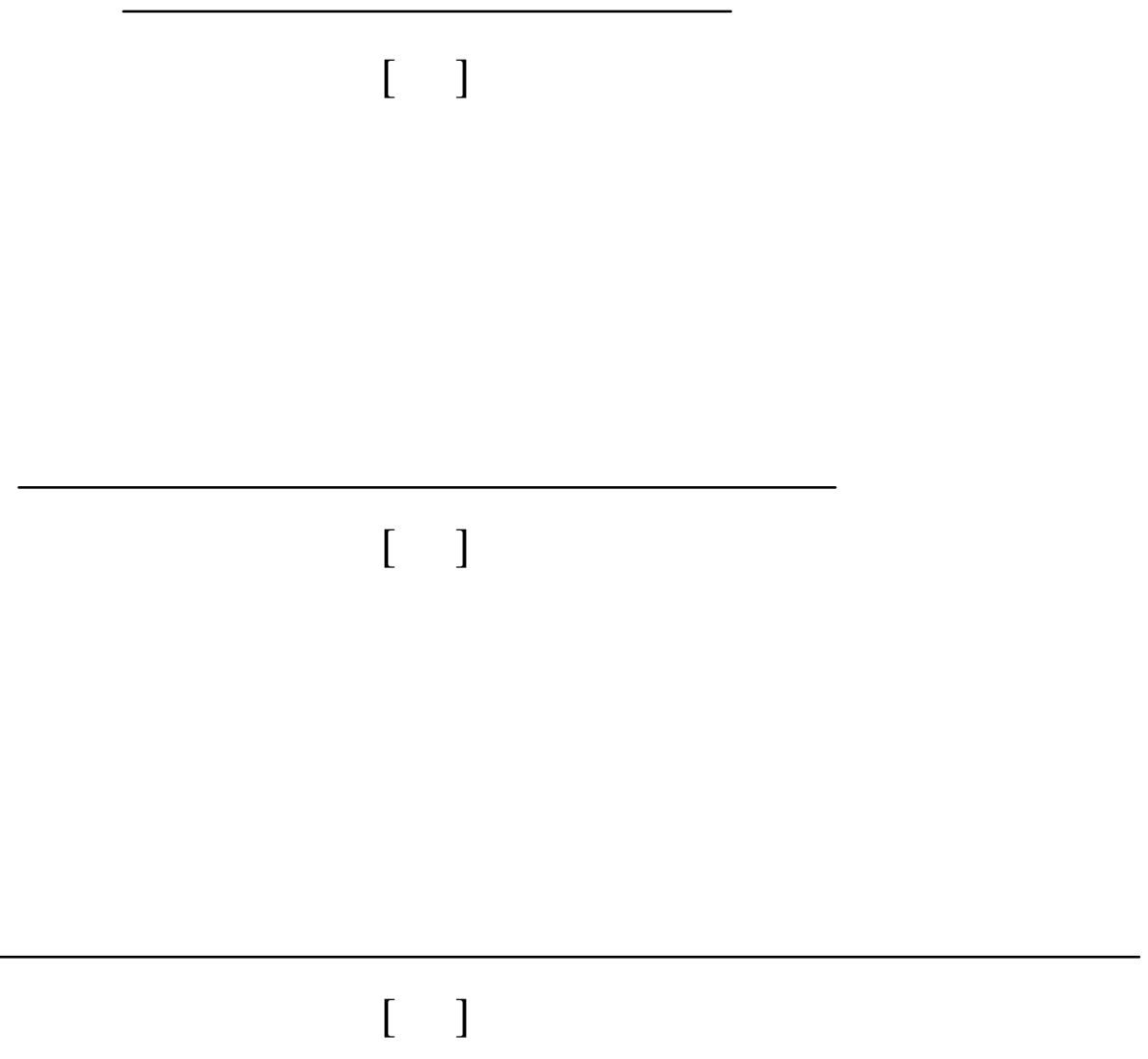

[ ] 
Participant R 01

\section{Clinical Judgment Faith Bias: \\ The Impact of Faith and Multicultural \\ Competence on Clinical Judgment \\ Pilot Study: Magnitude Estimation Scaling}

\section{Booklet 2: \\ Line Drawing}

[Given to both Groups of College Students] 
Participant R 01

\section{Magnitude Estimation Scaling: Line Drawing}

Now you are going to do something a little different. Please leaf through the next couple pages of this booklet and note that some of the numbers are larger than others. For each number, your task is to draw a line. Again, the first number is your reference. It is the number 50 , and a line has been drawn above it.

\section{[50 ]}

The line above the number 50 is your reference. All you need to do is draw a line above each number given. The bigger the number given compared to the reference number of 50 , the longer the line you will draw. For example, if the number 100 is given, this is twice the number 50, so you would draw a line about two times longer than the reference line. If the number 500 is given, you would draw a line ten times longer. On the other hand, if the number 25 is given, this is half the number 50, so you would draw a line about one half as long as the reference line. For the number 5, you would draw a line about one tenth as long. Give each number a line, whatever seems appropriate, to express how the number compares to the reference number: The bigger the number, the longer your line compared to the reference line. The smaller the number, the shorter the line compared to the reference line. Again, no rulers, please. I am only interested in your general impressions. 


\section{Participant R 01}

[ 26]

[ 74 ]

[ 4 ]

[ 98 ] 


\section{Participant R 01}

[38 ]

[ 2 ]

[14 ]

[ 18 ] 


\section{Participant R 01}

[64]

[ 86 ]

[ 150 ]

[ 200 ] 
Participant R 01

\section{Clinical Judgment Faith Bias: \\ The Impact of Faith and Multicultural \\ Competence on Clinical Judgment \\ Pilot Study: Magnitude Estimation Scaling}

\section{Booklet 3: \\ Religious Vignette Number Estimation}

[Given to the Religious Vignette Group of College Students Only] 
Participant R 01

\section{Magnitude Estimation Scaling: Religious Vignette Number Estimation}

Now that you have practiced using numbers and drawing lines, I would like to ask your opinion about how religious you think certain people are by having you assign numbers to them. The following pages contain a series of one-paragraph vignettes describing religious people. Some of the vignettes describe very religious people, and some of the vignettes describe not very religious people. The first vignette is your Reference Vignette:

Mary describes herself as somewhat religious, stating that she believes in God and has a personal relationship with Jesus. She refers to her faith as something she has always believed in because it is familiar and feels right to her. She enjoys spending time with others of her denomination, listens intently to sermons on Sundays, and frequently socializes with her church friends. She prays to God a few times each week and sometimes reads the Bible. She occasionally donates money to charities that preach Biblical teachings and establish churches in underserved mission communities. She believes everyone is entitled to their own religious beliefs and can live their lives as they choose, though she also feels that Christianity is the only religion that reveals God and is truly from God.

This vignette has been assigned a numerical value of 50:

\section{[50]}

Use this Reference Vignette to judge all the others. For all of the vignettes on the following pages, you are to use numbers to indicate how religious each vignette is compared to the Reference Vignette. For example, if you think that a vignette is twice as religious as the Reference Vignette, then you should give that vignette a numerical value of 100. On the other hand, if you think that a vignette is half as religious as the Reference Vignette, then you should give that vignette a numerical value of 25 . There is no upper limit; use any number so long as it shows how religious you think the vignette is. If you think that something is not religious, give it a 0 . 


\section{Participant R 01}

\section{$\underline{\text { Vignette } 1}$}

Mary considers herself to be a very devout Christian and describes herself as a person whose entire life revolves around Jesus. She states that her faith is more than just a belief system - it is a fundamental part of her individual identity, family life, and cultural upbringing. She spends much of her time doing church-related activities: she attends biweekly meetings for her church's finance committee, she attends church choir practice, and, on Sundays, she sings at two morning church services, teaches Sunday school, and goes to a smaller evening service. She has memorized hundreds of quotations from the Bible, and whenever she needs to make a decision about something, she thinks of the lessons she has learned from the many sermons she has heard and scriptures she has read, and then she asks herself, "What would Jesus do?" She gives liberally to youth ministry activities, foreign missions, local outreach ministries, and charitable organizations that her church sponsors. She freely shares her Christian faith with others and lives her life as a testimony of her faith in Christ.

\section{Vignette 2}

Mary considers herself to be a "nonreligious" person. She believes in God and the revelations of the Bible, but she feels that living a moral life is more important than attending a church. She says that the only times she attended church in her life were for a wedding and two funerals. She occasionally reads about religion, but she says she has never understood why religion is so important to so many people. She feels that it is important and right to live a moral life, to help others in need, and to give to charities, even if only occasionally. She believes in open dialogue between individuals regardless of their religious or denominational affiliation, but ever since she was a teenager, Mary has been suspicious of people who preach to others or tell others what they should believe. 


\section{Participant R 01}

\section{$\underline{\text { Vignette } 3}$}

Mary considers herself to be an evangelical Christian and describes herself as a very religious person whose close personal relationship with Jesus Christ influences every area of her life. She believes that it is important for her to attend church regularly and places great emphasis on the sermons she hears there. She keeps well informed about her local church and regularly attends committee meetings to influence decisions that her church makes. At home, she prays to God several times a day in a respectful prayer attitude, and she often piously studies the Bible for an hour or two each day. She donates both time and money to several charitable organizations that her church sponsors. At work, she actively gives testimony of her faith in Christ with her coworkers, especially with those who do not regularly attend church, in an effort to get them to consider attending church or at least to tell them about the possibilities of an experience with Jesus.

\section{[ ]}

\section{Vignette 4}

Mary considers herself to be a Christian and describes herself as a somewhat religious person who believes in God. She believes that the teachings in the Bible represent the truth and have practical value in the modern world; she interprets them as metaphors and parables to be interpreted and applied to her everyday life. She attends church services occasionally, and, when she does, she sings, prays, and learns something from the sermon, often feeling the presence of the Lord with her. She prefers to worship quietly and privately, by praying to God a few times a week and occasionally reading the Bible. She occasionally tithes a percentage of her income to her church. She refers to her beliefs as feeling right for her but says she can understand why other people might believe differently than she does. 


\section{Participant R 01}

\section{$\underline{\text { Vignette } 5}$}

Mary considers herself to be a devout Christian and describes herself as a very religious person with a close personal relationship with Jesus Christ. Religion is especially important to her because it answers many questions for her about the meaning of life. She attends church 3-4 times each week, and, at a typical church service, she enters into worship and praise. She memorizes verses from the Bible and prays to God several times each day. She faithfully gives a percentage of her income to the church. She believes that her religion is the one true faith and enthusiastically gives testimony of her faith in Jesus whenever the topic of her religion comes up in conversation.

\section{$\underline{\text { Vignette } 6}$}

Mary considers herself to be a born-again Christian and describes herself as "truly devoted to Jesus." She views Jesus as the source of her strength and happiness, both directly and indirectly. The church services she attends typically last 3 or 4 hours and involve singing, giving testimony, and praising Jesus. Privately, she prays and reads the Bible several times a day, decorates her home with religious items, and talks to others about what she believes. She volunteers two or three times a month at a local homeless shelter and often gives what little money she has to her church to support its overseas missions and foreign ministries, seeing her community involvement as "my privilege as a true Christian woman." She feels strongly that "it's not enough for me just to sit in the pew - I need to be involved in meeting the needs others have of clothing, shelter, food, and friendship in order to truly serve the Lord." 


\section{Participant R 01}

\section{$\underline{\text { Vignette } 7}$}

Mary used to be a Christian but no longer considers herself to be a religious person. She believes in God, the Bible, and following the golden rule. She used to attend church occasionally, but she has not done so in ten years. She also used to pray every day, and on rare occasions she still prays privately, but she does so very infrequently. At work, she gives the minimum pledge allowed by her company to the United Way. She believes everyone is entitled to their own religious beliefs and can live their lives as they choose.

\section{$\underline{\text { Vignette } 8}$}

Mary describes herself as somewhat religious, stating that she believes in God and has a personal relationship with Jesus. She refers to her faith as something she has always believed in because it is familiar and feels right to her. She enjoys spending time with others of her denomination, listens intently to sermons on Sundays, and frequently socializes with her church friends. She prays to God a few times each week and sometimes reads the Bible. She occasionally donates money to charities that preach Biblical teachings and establish churches in underserved mission communities. She believes everyone is entitled to their own religious beliefs and can live their lives as they choose, though she also feels that Christianity is the only religion that reveals God and is truly from God. 


\section{Participant R 01}

\section{$\underline{\text { Vignette } 9}$}

Mary considers herself to be somewhat religious and describes her religion as important to her. While she considers herself to be a Christian, she thinks it is more important to live a good life than to be a member of a particular church, though she also believes church attendance to be important. She attends church services about twice a month, where she participates actively and listens attentively to the sermon. She spends time trying to grow in understanding of her faith and often reads books and magazines about her faith. She sometimes does charity work on the weekends, volunteering at a local homeless shelter, to which she also donates used clothing, furniture, and other household items when she no longer needs them. She occasionally tells others about her beliefs if they seem receptive, but she does not "preach" to others, not wanting to offend them.

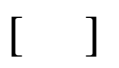

\section{$\underline{\text { Vignette } 10}$}

Mary considers herself to be a Christian but describes herself as not very religious - "I just believe in Christ and the Bible." She views herself as a moral and virtuous person, though she believes that she should attend church more often than she does. She attends church on Easter and around Christmas time and says she does so "to learn from the two most important sermons each year," and because she feels that she cannot be a Christian and never attend church. She has given considerable reflection to what she believes, and she occasionally has conversations with God whenever she feels the need to get a clearer perspective on her life. She occasionally volunteers at a local animal shelter and donates to Christian outreach ministries. She tells people she is Christian if they ask her and sometimes talks about religion with others, but she also believes everyone is entitled to their own religious beliefs and should live their lives as they choose. 


\section{Participant R 01}

\section{$\underline{\text { Vignette } 11}$}

Mary considers herself to be an avid mathematician and describes herself as a person whose entire life revolves around numbers. She states that her love of math is more than just her day job - it is a fundamental part of her individual identity, and how she makes sense out of the world. She spends much of her time doing math-related activities: she works as an accountant, attends biweekly meetings of the local university's mathematics club, competes in Math Bowl competitions every summer, and audits at least one graduate-level math course per semester. She has memorized Pi to the two hundredth decimal place, competes with mathematicians around the world online to solve various mathematics puzzles, and does Sudoku puzzles nearly every night. She volunteers at two local charities, giving them free accounting advice and helping them keep track of their accounts. She often talks with other people about numbers and freely shares her knowledge of mathematics and accounting with her friends, living her life "by the numbers and loving it."

\section{$\underline{\text { Vignette } 12}$}

Mary considers herself to be a devout Baptist woman and describes herself as a person whose entire life revolves around her church. She views her congregation and the relationship she has with Christ as the source of her strength and happiness. She attends church at least twice a week and enjoys singing, praising God, learning from her pastor's weekly messages, and enjoying fellowship with her Christian friends. Privately, she places great emphasis on the sermons she hears, reflecting on their meaning and trying her best to apply them to her Christian life. She frequently donates money to charities that preach Biblical teachings following the Baptist traditions and establish churches in underserved mission communities; she faithfully tithes a percentage of her income to her church. In this way, she lives her life as a testimony of her faith in Christ and freely shares her Christian faith with others whenever she feels it is appropriate. 


\section{Participant R 01}

\section{Vignette 13}

Mary describes religion as something she turns to in moments of despair. She says that she has experienced "the life-giving nature of the word of God," that she used to believe that church attendance was important, and that she used to place conviction in "the truths of my faith," but she is no longer certain of this conviction. She used to attend church occasionally but rarely finds the time to do so any more. She has read most of the Bible and thought it sounded true, but she found it to be somewhat dry and boring. At work, she gives to the Salvation Army or other legitimate charities at least once each year. She is most familiar with Christian teachings but says she is open-minded towards the teachings of all faiths.

\section{$\underline{\text { Vignette } 14}$}

Mary considers herself to be a Christian and believes in Biblical teachings, but she also considers herself to be a skeptic who does not accept what she hears in regards to her religious beliefs without first questioning its validity. She describes attending church as something she occasionally does because it is "an important thing to do once in awhile," and because she believes it is important for her to learn occasionally from the lessons her pastor has to teach. She attends church about once a month. She prays to God sometimes, and she has read several passages from the Bible. Almost every year, around Christmas time, she volunteers at an annual soup kitchen which her church sponsors. She says that her beliefs seem to her to be true, but that she is not absolutely certain - "I could be wrong about what I believe." 
Participant R 01

\section{Clinical Judgment Faith Bias: \\ The Impact of Faith and Multicultural \\ Competence on Clinical Judgment \\ Pilot Study: Magnitude Estimation Scaling}

\section{Booklet 4: \\ Religious Vignette Line Drawing}

[Given to the Religious Vignette Group of College Students Only] 
Participant R 01

\section{Magnitude Estimation Scaling: Religious Vignette Line Drawing}

Now that you have assigned numbers to the different vignettes, your task will be to express your opinion about how religious you think these people are by drawing lines for each vignette. The following pages contain the same series of one-paragraph vignettes you just assigned numbers to. As before, the first vignette is your Reference Vignette:

Mary describes herself as somewhat religious, stating that she believes in God and has a personal relationship with Jesus. She refers to her faith as something she has always believed in because it is familiar and feels right to her. She enjoys spending time with others of her denomination, listens intently to sermons on Sundays, and frequently socializes with her church friends. She prays to God a few times each week and sometimes reads the Bible. She occasionally donates money to charities that preach Biblical teachings and establish churches in underserved mission communities. She believes everyone is entitled to their own religious beliefs and can live their lives as they choose, though she also feels that Christianity is the only religion that reveals God and is truly from God.

This vignette has been assigned a medium length line:

Use this Reference Vignette to judge all the others. For all of the vignettes on the following pages, you are to draw lines to indicate how religious each vignette is compared to the Reference Vignette. For example, if you think that a vignette is twice as religious as the Reference Vignette, then you should draw a line that is twice as long as the medium length line above. On the other hand, if you think that a vignette is half as religious as the Reference Vignette, then you should draw a line that is half as long as the medium length line above. There is no upper limit; draw any length line so long as it shows how religious you think the vignette is. If you think that something is not religious, draw a very, very short line, or give it a 0 . 


\section{Participant R 01}

\section{$\underline{\text { Vignette } 1}$}

Mary considers herself to be a very devout Christian and describes herself as a person whose entire life revolves around Jesus. She states that her faith is more than just a belief system - it is a fundamental part of her individual identity, family life, and cultural upbringing. She spends much of her time doing church-related activities: she attends biweekly meetings for her church's finance committee, she attends church choir practice, and, on Sundays, she sings at two morning church services, teaches Sunday school, and goes to a smaller evening service. She has memorized hundreds of quotations from the Bible, and whenever she needs to make a decision about something, she thinks of the lessons she has learned from the many sermons she has heard and scriptures she has read, and then she asks herself, "What would Jesus do?" She gives liberally to youth ministry activities, foreign missions, local outreach ministries, and charitable organizations that her church sponsors. She freely shares her Christian faith with others and lives her life as a testimony of her faith in Christ.

\section{Vignette 2}

Mary considers herself to be a "nonreligious" person. She believes in God and the revelations of the Bible, but she feels that living a moral life is more important than attending a church. She says that the only times she attended church in her life were for a wedding and two funerals. She occasionally reads about religion, but she says she has never understood why religion is so important to so many people. She feels that it is important and right to live a moral life, to help others in need, and to give to charities, even if only occasionally. She believes in open dialogue between individuals regardless of their religious or denominational affiliation, but ever since she was a teenager, Mary has been suspicious of people who preach to others or tell others what they should believe. 


\section{Participant R 01}

\section{$\underline{\text { Vignette } 3}$}

Mary considers herself to be an evangelical Christian and describes herself as a very religious person whose close personal relationship with Jesus Christ influences every area of her life. She believes that it is important for her to attend church regularly and places great emphasis on the sermons she hears there. She keeps well informed about her local church and regularly attends committee meetings to influence decisions that her church makes. At home, she prays to God several times a day in a respectful prayer attitude, and she often piously studies the Bible for an hour or two each day. She donates both time and money to several charitable organizations that her church sponsors. At work, she actively gives testimony of her faith in Christ with her coworkers, especially with those who do not regularly attend church, in an effort to get them to consider attending church or at least to tell them about the possibilities of an experience with Jesus.

\section{$\underline{\text { Vignette } 4}$}

Mary considers herself to be a Christian and describes herself as a somewhat religious person who believes in God. She believes that the teachings in the Bible represent the truth and have practical value in the modern world; she interprets them as metaphors and parables to be interpreted and applied to her everyday life. She attends church services occasionally, and, when she does, she sings, prays, and learns something from the sermon, often feeling the presence of the Lord with her. She prefers to worship quietly and privately, by praying to God a few times a week and occasionally reading the Bible. She occasionally tithes a percentage of her income to her church. She refers to her beliefs as feeling right for her but says she can understand why other people might believe differently than she does. 


\section{Participant R 01}

\section{$\underline{\text { Vignette } 5}$}

Mary considers herself to be a devout Christian and describes herself as a very religious person with a close personal relationship with Jesus Christ. Religion is especially important to her because it answers many questions for her about the meaning of life. She attends church 3-4 times each week, and, at a typical church service, she enters into worship and praise. She memorizes verses from the Bible and prays to God several times each day. She faithfully gives a percentage of her income to the church. She believes that her religion is the one true faith and enthusiastically gives testimony of her faith in Jesus whenever the topic of her religion comes up in conversation.

\section{$\underline{\text { Vignette } 6}$}

Mary considers herself to be a born-again Christian and describes herself as "truly devoted to Jesus." She views Jesus as the source of her strength and happiness, both directly and indirectly. The church services she attends typically last 3 or 4 hours and involve singing, giving testimony, and praising Jesus. Privately, she prays and reads the Bible several times a day, decorates her home with religious items, and talks to others about what she believes. She volunteers two or three times a month at a local homeless shelter and often gives what little money she has to her church to support its overseas missions and foreign ministries, seeing her community involvement as "my privilege as a true Christian woman." She feels strongly that "it's not enough for me just to sit in the pew - I need to be involved in meeting the needs others have of clothing, shelter, food, and friendship in order to truly serve the Lord." 


\section{Participant R 01}

\section{Vignette 7}

Mary used to be a Christian but no longer considers herself to be a religious person. She believes in God, the Bible, and following the golden rule. She used to attend church occasionally, but she has not done so in ten years. She also used to pray every day, and on rare occasions she still prays privately, but she does so very infrequently. At work, she gives the minimum pledge allowed by her company to the United Way. She believes everyone is entitled to their own religious beliefs and can live their lives as they choose.

\section{Vignette 8}

Mary describes herself as somewhat religious, stating that she believes in God and has a personal relationship with Jesus. She refers to her faith as something she has always believed in because it is familiar and feels right to her. She enjoys spending time with others of her denomination, listens intently to sermons on Sundays, and frequently socializes with her church friends. She prays to God a few times each week and sometimes reads the Bible. She occasionally donates money to charities that preach Biblical teachings and establish churches in underserved mission communities. She believes everyone is entitled to their own religious beliefs and can live their lives as they choose, though she also feels that Christianity is the only religion that reveals God and is truly from God. 


\section{Participant R 01}

\section{$\underline{\text { Vignette } 9}$}

Mary considers herself to be somewhat religious and describes her religion as important to her. While she considers herself to be a Christian, she thinks it is more important to live a good life than to be a member of a particular church, though she also believes church attendance to be important. She attends church services about twice a month, where she participates actively and listens attentively to the sermon. She spends time trying to grow in understanding of her faith and often reads books and magazines about her faith. She sometimes does charity work on the weekends, volunteering at a local homeless shelter, to which she also donates used clothing, furniture, and other household items when she no longer needs them. She occasionally tells others about her beliefs if they seem receptive, but she does not "preach" to others, not wanting to offend them.

\section{$\underline{\text { Vignette } 10}$}

Mary considers herself to be a Christian but describes herself as not very religious - "I just believe in Christ and the Bible." She views herself as a moral and virtuous person, though she believes that she should attend church more often than she does. She attends church on Easter and around Christmas time and says she does so "to learn from the two most important sermons each year," and because she feels that she cannot be a Christian and never attend church. She has given considerable reflection to what she believes, and she occasionally has conversations with God whenever she feels the need to get a clearer perspective on her life. She occasionally volunteers at a local animal shelter and donates to Christian outreach ministries. She tells people she is Christian if they ask her and sometimes talks about religion with others, but she also believes everyone is entitled to their own religious beliefs and should live their lives as they choose. 


\section{Participant R 01}

\section{$\underline{\text { Vignette } 11}$}

Mary considers herself to be an avid mathematician and describes herself as a person whose entire life revolves around numbers. She states that her love of math is more than just her day job - it is a fundamental part of her individual identity, and how she makes sense out of the world. She spends much of her time doing math-related activities: she works as an accountant, attends biweekly meetings of the local university's mathematics club, competes in Math Bowl competitions every summer, and audits at least one graduate-level math course per semester. She has memorized Pi to the two hundredth decimal place, competes with mathematicians around the world online to solve various mathematics puzzles, and does Sudoku puzzles nearly every night. She volunteers at two local charities, giving them free accounting advice and helping them keep track of their accounts. She often talks with other people about numbers and freely shares her knowledge of mathematics and accounting with her friends, living her life "by the numbers and loving it."

\section{$\underline{\text { Vignette } 12}$}

Mary considers herself to be a devout Baptist woman and describes herself as a person whose entire life revolves around her church. She views her congregation and the relationship she has with Christ as the source of her strength and happiness. She attends church at least twice a week and enjoys singing, praising God, learning from her pastor's weekly messages, and enjoying fellowship with her Christian friends. Privately, she places great emphasis on the sermons she hears, reflecting on their meaning and trying her best to apply them to her Christian life. She frequently donates money to charities that preach Biblical teachings following the Baptist traditions and establish churches in underserved mission communities; she faithfully tithes a percentage of her income to her church. In this way, she lives her life as a testimony of her faith in Christ and freely shares her Christian faith with others whenever she feels it is appropriate. 


\section{Participant R 01}

\section{$\underline{\text { Vignette } 13}$}

Mary describes religion as something she turns to in moments of despair. She says that she has experienced "the life-giving nature of the word of God," that she used to believe that church attendance was important, and that she used to place conviction in "the truths of my faith," but she is no longer certain of this conviction. She used to attend church occasionally but rarely finds the time to do so any more. She has read most of the Bible and thought it sounded true, but she found it to be somewhat dry and boring. At work, she gives to the Salvation Army or other legitimate charities at least once each year. She is most familiar with Christian teachings but says she is open-minded towards the teachings of all faiths.

\section{$\underline{\text { Vignette } 14}$}

Mary considers herself to be a Christian and believes in Biblical teachings, but she also considers herself to be a skeptic who does not accept what she hears in regards to her religious beliefs without first questioning its validity. She describes attending church as something she occasionally does because it is "an important thing to do once in awhile," and because she believes it is important for her to learn occasionally from the lessons her pastor has to teach. She attends church about once a month. She prays to God sometimes, and she has read several passages from the Bible. Almost every year, around Christmas time, she volunteers at an annual soup kitchen which her church sponsors. She says that her beliefs seem to her to be true, but that she is not absolutely certain - "I could be wrong about what I believe." 
Participant S 01

\section{Clinical Judgment Faith Bias: \\ The Impact of Faith and Multicultural Competence on Clinical Judgment \\ Pilot Study: Magnitude Estimation Scaling}

\section{Booklet 3: \\ Spiritual Vignette Number Estimation}

[Given to the Spiritual Vignette Group of College Students Only] 
Participant S 01

\section{Magnitude Estimation Scaling: Spiritual Vignette Number Estimation}

Now that you have practiced using numbers and drawing lines, I would like to ask your opinion about how spiritual you think certain people are by having you assign numbers to them. The following pages contain a series of one-paragraph vignettes describing spiritual people. Some of the vignettes describe very spiritual people, and some of the vignettes describe not very spiritual people. The first vignette is your Reference Vignette:

Mary describes herself as a somewhat spiritual person who considers herself to be an open-minded seeker. She describes her faith as "a kind of relationship I have with the universe," and she refers to her faith as something she believes in because it feels right to her. On occasion, she attends chant rituals, guided meditations, or spiritual healing services, out of curiosity to hear about the spiritual experiences of others. She prays to a divine being a few times each week, and she privately meditates about twice a month. Sometimes, she volunteers for the American Red Cross whenever they organize fundraisers and local community outreach activities in her neighborhood. She occasionally invites others to come to the spiritual healing services she attends if they ask her about what she believes, but she only does this if they seem interested in spiritual matters and bring up the topic first.

This vignette has been assigned a numerical value of 50:

[50]

Use this Reference Vignette to judge all the others. For all of the vignettes on the following pages, you are to use numbers to indicate how spiritual each vignette is compared to the Reference Vignette. For example, if you think that a vignette is twice as spiritual as the Reference Vignette, then you should give that vignette a numerical value of 100. On the other hand, if you think that a vignette is half as spiritual as the Reference Vignette, then you should give that vignette a numerical value of 25. There is no upper limit; use any number so long as it shows how spiritual you think the vignette is. If you think that something is not spiritual, give it a 0 . 


\section{Participant S 01}

\section{$\underline{\text { Vignette } 1}$}

Mary considers herself to be a spiritual seeker and describes herself as "truly devoted to the divine." She describes her faith as a close personal relationship among herself, the divine, like-minded others, and the universe itself, referring to it as an internalized, heartfelt kind of belief that she chose because "it just makes sense." She attends weekly meditation services that typically last for 3 or 4 hours and involve mantra, visualization, and clearing the mind of worldly concerns. Privately, she prays and reads spiritual or religious texts from diverse traditions several times a day, decorates her home with items from several different faiths, and talks to others about what she believes. She volunteers two or three times a month at a local homeless shelter and often gives what little money she has to girls and boys club programs to support their youth development projects, seeing her community involvement as "my privilege as a truly spiritual woman." She feels that "it's not enough for me just to sit around and read about this stuff - I need to be involved in order to truly serve the universe."

\section{Vignette 2}

Mary considers herself to be a "non-spiritual" person. She believes in following the golden rule, and she feels that living a moral life is more important than worshipping a higher power. She says that the only times she attended "spiritual events" in her life were for a wedding, two funerals, and a Yoga class. She occasionally reads about spirituality, but she says she has never understood why religion is so important to so many people. She feels that it is good to live a moral life, to help others in need, and to give to charities occasionally. She believes in open dialogue between individuals, but ever since she was a teenager, Mary has been suspicious of people who preach to others or tell others what they should believe. 


\section{Participant S 01}

\section{Vignette 3}

Mary considers herself to be "a mystical seeker" who is "in touch with the universe," and she describes herself as a very spiritual person whose close personal relationship with the divine influences every area of her life. She describes her faith as an intensely close and personal relationship with the divine and with the universe itself, referring to it as an internalized, heartfelt kind of belief that she believes in because it feels right to her. She typically worships by meditating quietly on her own, or occasionally by attending guided meditations with other people who regard spirituality as important. She meditates at least 3-4 times a week, and prays to a higher power each morning and night. She regularly volunteers for the American Red Cross and helps them organize fundraisers and local community outreach activities in her neighborhood. She often invites people she knows to join the spiritual healing services she attends, feeling that everyone should have the opportunity to "get in touch with themselves and the universe as I have."

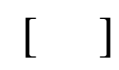

$\underline{\text { Vignette } 4}$

Mary considers herself to be a seeker and describes herself as a somewhat spiritual person who believes in the divine. She feels a sense of trust and security because she has a general feeling that "the universe provides." She attends spiritual healing services occasionally, and, when she does, she often feels the presence of the divine with her. She prefers to worship quietly and privately, by praying to a divine power a few times a week and occasionally reading books about religion or spirituality. She faithfully gives a percentage of each of her paychecks to the United Way. She refers to her beliefs as feeling right for her but says she can understand why other people might believe differently than she does. 


\section{Participant S 01}

\section{$\underline{\text { Vignette } 5}$}

Mary considers herself to be a "mystical seeker" and feels that her relationship with the divine and with the environment influences every area of her life. Her spirituality is especially important to her because it gives her a general sense about the meaning of life. She attends weekly spiritual healing services and has not missed a meeting in the past 5 years. She spends periods of time in private thought and meditation and makes sure that her behavior reflects the core teachings that the major world faiths have in common. Nearly every weekend, she volunteers at a local homeless shelter or an environmental preservation society. She often talks about her beliefs with her friends, neighbors, and coworkers, and, whenever she feels it is appropriate, she gives testimony of her experiences with meditation in an effort to get others to consider meditating themselves.

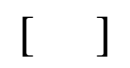

$\underline{\text { Vignette } 6}$

Mary considers herself to be a "mystical seeker" and describes herself as a very spiritual person whose close personal relationship with the universe influences every area of her life. She states that her faith is more than just a belief system - it is a fundamental part of her individual identity, family life, and cultural upbringing. She typically worships by attending mantra workshops - chant rituals that last for half a day or more - noting that at times this experience is incredibly powerful for her. During the rest of the week, she often privately talks to a higher power to make sure her life is on track. She regularly gives a generous percentage of her income to local charities, and just last year she donated an additional $\$ 2000$ towards the purchase of a new stove for a nearby homeless shelter. She often talks to others about what she believes, and makes it a point to tell others what an active role that her personal relationship with the universe has taken in guiding her life since she "realized the truth of the universe." 


\section{Participant S 01}

\section{$\underline{\text { Vignette } 7}$}

Mary used to be a spiritual person but no longer considers herself to be spiritual. She believes that it is not necessary for her to feel spiritual in order to live her life and to be happy. She used to attend spiritual healing services occasionally, but she has not done so in ten years. She also used to meditate, and, on rare occasions, she still prays to the divine privately, but she does so very infrequently, and she has not meditated in at least ten years. At work, she gives the minimum pledge allowed by her company to the United Way. She believes everyone is entitled to their own spiritual beliefs and can live their lives as they choose.

\section{Vignette 8}

Mary describes herself as a somewhat spiritual person who considers herself to be an open-minded seeker. She describes her faith as "a kind of relationship I have with the universe," and she refers to her faith as something she believes in because it feels right to her. On occasion, she attends chant rituals, guided meditations, or spiritual healing services, out of curiosity to hear about the spiritual experiences of others. She prays to a divine being a few times each week, and she privately meditates about twice a month. Sometimes, she volunteers for the American Red Cross whenever they organize fundraisers and local community outreach activities in her neighborhood. She occasionally invites others to come to the spiritual healing services she attends if they ask her about what she believes, but she only does this if they seem interested in spiritual matters and bring up the topic first. 


\section{Participant S 01}

\section{$\underline{\text { Vignette } 9}$}

Mary considers herself to be somewhat spiritual and describes her faith as important to her. She feels a sense of trust and security because she is hopeful that the universe will show her the right way to live her life. She attends a personal growth group on spiritual issues about twice a month, where she sits quietly and listens attentively to the personal spiritual experiences that others share. She spends time trying to grow in understanding of the universe and often reads books and magazines about spirituality. She sometimes does charity work on the weekends, volunteering at a local homeless shelter, to which she also donates clothing, furniture, and other household items when she no longer needs them. She occasionally tells others about her spiritual beliefs if they seem receptive, but she does not "preach" to others, not wanting to offend them.

\section{$\underline{\text { Vignette } 10}$}

Mary considers herself to be a person of faith, but she does not accept what she hears in regards to her beliefs without first questioning its validity. She views herself as "a little bit spiritual" and believes in a divine being, but she also believes that it is not necessary for her to act in certain ways or follow prescribed rituals in order to be a good or spiritual person. She used to attend a biweekly meditation class, but she has not found time to attend for more than a year. She has given considerable reflection to what she believes, and she occasionally has "conversations with the universe" whenever she feels the need to get a clearer perspective on her life. She occasionally volunteers at a local animal shelter and donates to nature conservation and preservation organizations. She says that her spiritual beliefs seem to be true, but that she is not absolutely certain - "I could be wrong about what I believe." 


\section{Participant S 01}

\section{$\underline{\text { Vignette } 11}$}

Mary considers herself to be an avid mathematician and describes herself as a person whose entire life revolves around numbers. She states that her love of math is more than just her day job - it is a fundamental part of her individual identity, and how she makes sense out of the world. She spends much of her time doing math-related activities: she works as an accountant, attends biweekly meetings of the local university's mathematics club, competes in Math Bowl competitions every summer, and audits at least one graduate-level math course per semester. She has memorized Pi to the two hundredth decimal place, competes with mathematicians around the world online to solve various mathematics puzzles, and does Sudoku puzzles nearly every night. She volunteers at two local charities, giving them free accounting advice and helping them keep track of their accounts. She often talks with other people about numbers and freely shares her knowledge of mathematics and accounting with her friends, living her life "by the numbers and loving it."

\section{$\underline{\text { Vignette } 12}$}

Mary considers herself to be a very spiritual person with a close personal relationship with the universe. She has always felt a sense of trust and security because she is certain that fate will show her the right way to live her life. She typically practices with others by attending chant rituals, guided meditations, and spiritual healing services. She memorizes verses from various spiritual and religious texts and prays to the divine several times each day. She faithfully gives a percentage of her income to charities. In this way, she lives her life as a testimony of her faith in the essential beauty and order of the universe and freely shares her spiritual beliefs with others whenever she feels it is appropriate. 


\section{Participant S 01}

\section{Vignette 13}

Mary considers herself to be open-minded, curious about the way the universe works, and "a little bit spiritual." She has felt moments of deep meaning in her life and connection to a higher power who has shown her the right way to live her life, though she states that the last time she experienced such a moment was ten years ago. Three or four times each year, she attends a meditation and Yoga workshop. She prays to a higher power sometimes, and she occasionally reflects on the meaning of life and her place in the universe. She donates to charity at least once or twice each year. She sometimes tells people that she believes in a higher power if the topic comes up, but she usually does not share her personal beliefs with other people.

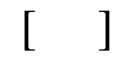

\section{$\underline{\text { Vignette } 14}$}

Mary considers herself to be "not very spiritual - I just believe in some kind of a higher power." She describes her faith as something which used to be important to her but which no longer has much relevance to her life any more. She attends meditation classes 2 or 3 times each month. She prays to a higher power sometimes, and she has read passages from several religious texts. She volunteers at a local homeless shelter around Thanksgiving almost every year. She tells people about her spiritual beliefs if they ask her, but she also believes everyone is entitled to their own beliefs and should live their lives as they choose. 
Participant S 01

\section{Clinical Judgment Faith Bias: \\ The Impact of Faith and Multicultural \\ Competence on Clinical Judgment \\ Pilot Study: Magnitude Estimation Scaling}

\section{Booklet 4: \\ Spiritual Vignette Line Drawing}

[Given to the Spiritual Vignette Group of College Students Only] 
Participant S 01

\section{Magnitude Estimation Scaling: Spiritual Vignette Line Drawing}

Now that you have assigned numbers to the different vignettes, your task will be to express your opinion about how spiritual you think these people are by drawing lines for each vignette. The following pages contain the same series of one-paragraph vignettes you just assigned numbers to. As before, the first vignette is your Reference Vignette:

Mary describes herself as a somewhat spiritual person who considers herself to be an open-minded seeker. She describes her faith as "a kind of relationship I have with the universe," and she refers to her faith as something she believes in because it feels right to her. On occasion, she attends chant rituals, guided meditations, or spiritual healing services, out of curiosity to hear about the spiritual experiences of others. She prays to a divine being a few times each week, and she privately meditates about twice a month. Sometimes, she volunteers for the American Red Cross whenever they organize fundraisers and local community outreach activities in her neighborhood. She occasionally invites others to come to the spiritual healing services she attends if they ask her about what she believes, but she only does this if they seem interested in spiritual matters and bring up the topic first.

This vignette has been assigned a medium length line:

Use this Reference Vignette to judge all the others. For all of the vignettes on the following pages, you are to draw lines to indicate how spiritual each vignette is compared to the Reference Vignette. For example, if you think that a vignette is twice as spiritual as the Reference Vignette, then you should draw a line that is twice as long as the medium length line above. On the other hand, if you think that a vignette is half as spiritual as the Reference Vignette, then you should draw a line that is half as long as the medium length line above. There is no upper limit; draw any length line so long as it shows how spiritual you think the vignette is. If you think that something is not spiritual, draw a very, very short line, or give it a 0 . 


\section{Participant S 01}

\section{$\underline{\text { Vignette } 1}$}

Mary considers herself to be a spiritual seeker and describes herself as "truly devoted to the divine." She describes her faith as a close personal relationship among herself, the divine, like-minded others, and the universe itself, referring to it as an internalized, heartfelt kind of belief that she chose because "it just makes sense." She attends weekly meditation services that typically last for 3 or 4 hours and involve mantra, visualization,

and clearing the mind of worldly concerns. Privately, she prays and reads spiritual or religious texts from diverse traditions several times a day, decorates her home with items from several different faiths, and talks to others about what she believes. She volunteers two or three times a month at a local homeless shelter and often gives what little money she has to girls and boys club programs to support their youth development projects, seeing her community involvement as "my privilege as a truly spiritual woman." She feels that "it's not enough for me just to sit around and read about this stuff - I need to be involved in order to truly serve the universe."

\section{Vignette 2}

Mary considers herself to be a "non-spiritual" person. She believes in following the golden rule, and she feels that living a moral life is more important than worshipping a higher power. She says that the only times she attended "spiritual events" in her life were for a wedding, two funerals, and a Yoga class. She occasionally reads about spirituality, but she says she has never understood why religion is so important to so many people. She feels that it is good to live a moral life, to help others in need, and to give to charities occasionally. She believes in open dialogue between individuals, but ever since she was a teenager, Mary has been suspicious of people who preach to others or tell others what they should believe. 


\section{Participant S 01}

\section{Vignette 3}

Mary considers herself to be "a mystical seeker" who is "in touch with the universe," and she describes herself as a very spiritual person whose close personal relationship with the divine influences every area of her life. She describes her faith as an intensely close and personal relationship with the divine and with the universe itself, referring to it as an internalized, heartfelt kind of belief that she believes in because it feels right to her. She typically worships by meditating quietly on her own, or occasionally by attending guided meditations with other people who regard spirituality as important. She meditates at least 3-4 times a week, and prays to a higher power each morning and night. She regularly volunteers for the American Red Cross and helps them organize fundraisers and local community outreach activities in her neighborhood. She often invites people she knows to join the spiritual healing services she attends, feeling that everyone should have the opportunity to "get in touch with themselves and the universe as I have."

\section{$\underline{\text { Vignette } 4}$}

Mary considers herself to be a seeker and describes herself as a somewhat spiritual person who believes in the divine. She feels a sense of trust and security because she has a general feeling that "the universe provides." She attends spiritual healing services occasionally, and, when she does, she often feels the presence of the divine with her. She prefers to worship quietly and privately, by praying to a divine power a few times a week and occasionally reading books about religion or spirituality. She faithfully gives a percentage of each of her paychecks to the United Way. She refers to her beliefs as feeling right for her but says she can understand why other people might believe differently than she does. 


\section{Participant S 01}

\section{$\underline{\text { Vignette } 5}$}

Mary considers herself to be a "mystical seeker" and feels that her relationship with the divine and with the environment influences every area of her life. Her spirituality is especially important to her because it gives her a general sense about the meaning of life. She attends weekly spiritual healing services and has not missed a meeting in the past 5 years. She spends periods of time in private thought and meditation and makes sure that her behavior reflects the core teachings that the major world faiths have in common. Nearly every weekend, she volunteers at a local homeless shelter or an environmental preservation society. She often talks about her beliefs with her friends, neighbors, and coworkers, and, whenever she feels it is appropriate, she gives testimony of her experiences with meditation in an effort to get others to consider meditating themselves.

\section{$\underline{\text { Vignette } 6}$}

Mary considers herself to be a "mystical seeker" and describes herself as a very spiritual person whose close personal relationship with the universe influences every area of her life. She states that her faith is more than just a belief system - it is a fundamental part of her individual identity, family life, and cultural upbringing. She typically worships by attending mantra workshops - chant rituals that last for half a day or more - noting that at times this experience is incredibly powerful for her. During the rest of the week, she often privately talks to a higher power to make sure her life is on track. She regularly gives a generous percentage of her income to local charities, and just last year she donated an additional $\$ 2000$ towards the purchase of a new stove for a nearby homeless shelter. She often talks to others about what she believes, and makes it a point to tell others what an active role that her personal relationship with the universe has taken in guiding her life since she "realized the truth of the universe." 


\section{Participant S 01}

\section{$\underline{\text { Vignette } 7}$}

Mary used to be a spiritual person but no longer considers herself to be spiritual. She believes that it is not necessary for her to feel spiritual in order to live her life and to be happy. She used to attend spiritual healing services occasionally, but she has not done so in ten years. She also used to meditate, and, on rare occasions, she still prays to the divine privately, but she does so very infrequently, and she has not meditated in at least ten years. At work, she gives the minimum pledge allowed by her company to the United Way. She believes everyone is entitled to their own spiritual beliefs and can live their lives as they choose.

\section{$\underline{\text { Vignette } 8}$}

Mary describes herself as a somewhat spiritual person who considers herself to be an open-minded seeker. She describes her faith as "a kind of relationship I have with the universe," and she refers to her faith as something she believes in because it feels right to her. On occasion, she attends chant rituals, guided meditations, or spiritual healing services, out of curiosity to hear about the spiritual experiences of others. She prays to a divine being a few times each week, and she privately meditates about twice a month. Sometimes, she volunteers for the American Red Cross whenever they organize fundraisers and local community outreach activities in her neighborhood. She occasionally invites others to come to the spiritual healing services she attends if they ask her about what she believes, but she only does this if they seem interested in spiritual matters and bring up the topic first. 


\section{Participant S 01}

\section{$\underline{\text { Vignette } 9}$}

Mary considers herself to be somewhat spiritual and describes her faith as important to her. She feels a sense of trust and security because she is hopeful that the universe will show her the right way to live her life. She attends a personal growth group on spiritual issues about twice a month, where she sits quietly and listens attentively to the personal spiritual experiences that others share. She spends time trying to grow in understanding of the universe and often reads books and magazines about spirituality. She sometimes does charity work on the weekends, volunteering at a local homeless shelter, to which she also donates clothing, furniture, and other household items when she no longer needs them. She occasionally tells others about her spiritual beliefs if they seem receptive, but she does not "preach" to others, not wanting to offend them.

\section{$\underline{\text { Vignette } 10}$}

Mary considers herself to be a person of faith, but she does not accept what she hears in regards to her beliefs without first questioning its validity. She views herself as "a little bit spiritual" and believes in a divine being, but she also believes that it is not necessary for her to act in certain ways or follow prescribed rituals in order to be a good or spiritual person. She used to attend a biweekly meditation class, but she has not found time to attend for more than a year. She has given considerable reflection to what she believes, and she occasionally has "conversations with the universe" whenever she feels the need to get a clearer perspective on her life. She occasionally volunteers at a local animal shelter and donates to nature conservation and preservation organizations. She says that her spiritual beliefs seem to be true, but that she is not absolutely certain - "I could be wrong about what I believe." 


\section{Participant S 01}

\section{Vignette 11}

Mary considers herself to be an avid mathematician and describes herself as a person whose entire life revolves around numbers. She states that her love of math is more than just her day job - it is a fundamental part of her individual identity, and how she makes sense out of the world. She spends much of her time doing math-related activities: she works as an accountant, attends biweekly meetings of the local university's mathematics club, competes in Math Bowl competitions every summer, and audits at least one graduate-level math course per semester. She has memorized Pi to the two hundredth decimal place, competes with mathematicians around the world online to solve various mathematics puzzles, and does Sudoku puzzles nearly every night. She volunteers at two local charities, giving them free accounting advice and helping them keep track of their accounts. She often talks with other people about numbers and freely shares her knowledge of mathematics and accounting with her friends, living her life "by the numbers and loving it."

\section{$\underline{\text { Vignette } 12}$}

Mary considers herself to be a very spiritual person with a close personal relationship with the universe. She has always felt a sense of trust and security because she is certain that fate will show her the right way to live her life. She typically practices with others by attending chant rituals, guided meditations, and spiritual healing services. She memorizes verses from various spiritual and religious texts and prays to the divine several times each day. She faithfully gives a percentage of her income to charities. In this way, she lives her life as a testimony of her faith in the essential beauty and order of the universe and freely shares her spiritual beliefs with others whenever she feels it is appropriate. 


\section{Participant S 01}

\section{Vignette 13}

Mary considers herself to be open-minded, curious about the way the universe works, and "a little bit spiritual." She has felt moments of deep meaning in her life and connection to a higher power who has shown her the right way to live her life, though she states that the last time she experienced such a moment was ten years ago. Three or four times each year, she attends a meditation and Yoga workshop. She prays to a higher power sometimes, and she occasionally reflects on the meaning of life and her place in the universe. She donates to charity at least once or twice each year. She sometimes tells people that she believes in a higher power if the topic comes up, but she usually does not share her personal beliefs with other people.

\section{Vignette 14}

Mary considers herself to be "not very spiritual - I just believe in some kind of a higher power." She describes her faith as something which used to be important to her but which no longer has much relevance to her life any more. She attends meditation classes 2 or 3 times each month. She prays to a higher power sometimes, and she has read passages from several religious texts. She volunteers at a local homeless shelter around Thanksgiving almost every year. She tells people about her spiritual beliefs if they ask her, but she also believes everyone is entitled to their own beliefs and should live their lives as they choose. 


\section{Religion Magnitude Estimation Scaling Demographic Information Questionnaire}

1.) Age: years old

2.) Sex: (check one) Male Female

3.) Race/Ethnicity: (check one) Caucasian/White _ Asian American African American/Black Native American Hispanic American/Latino(a) Other*

* Please Specify:

4.) Year in School Freshman Sophomore Junior Biracial/Multiracial* Senior

Fifth Year (or more) Undergraduate Masters Student Doctoral Student

5.) What was your predominant religious background? (check one)

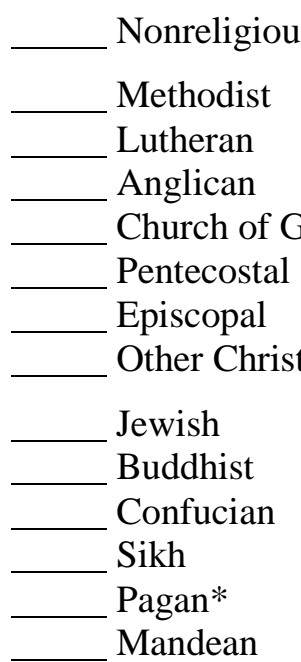

Atheist
Roman Catholic
Southern Baptist
Presbyterian
Church of Christ
Assembly of God
LDS/Mormon

Agnostic Christian Orthodox Other Baptist* Seventh Day Adventist Church of God in Christ Church of the Nazarene Jehovah's Witness

Church*

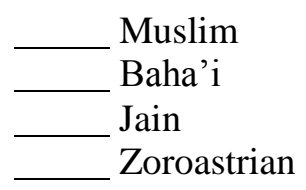
Shaman* Unitarian Universalist
Hindu

Taoist Shintoist Chinese Religionist Ethnic/Tribal Religionist* Mandean Other Religious Background*

* Please Specify: 
6.) Describe yourself today. (check as many as apply)

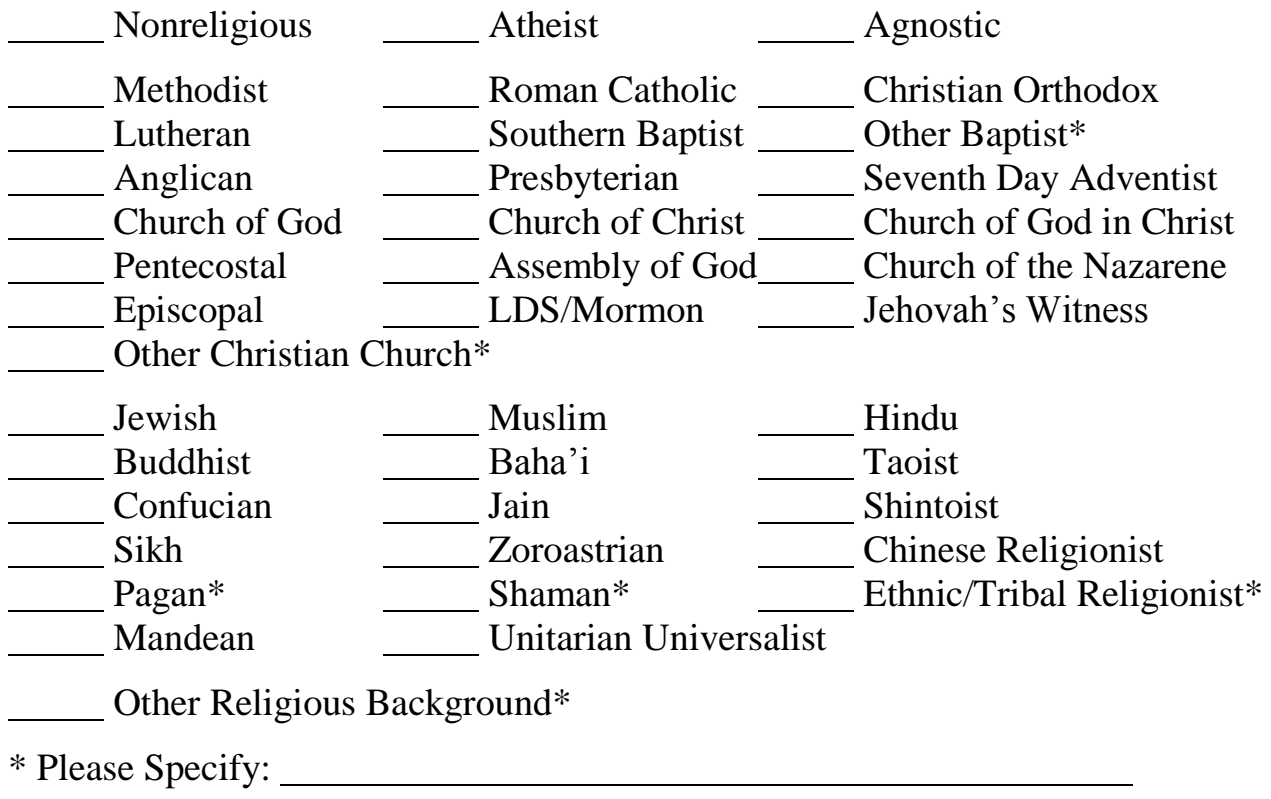

7.) Are you participating in any organized religious body now? No Yes Where?

How often? 


\section{Spirituality Magnitude Estimation Scaling Demographic Information Questionnaire}

8.) Age: years old

9.) Sex: (check one) Male Female

10.) Race/Ethnicity: (check one) Caucasian/White _ Asian American African American/Black Hispanic American/Latino(a) Native American Biracial/Multiracial* Other*

* Please Specify:

11.) Year in School Freshman Sophomore Junior Fifth Year (or more) Undergraduate Masters Student Senior Doctoral Student

12.) What was your predominant religious background? (check one)

\begin{tabular}{|c|c|c|}
\hline Nonreligious & Atheist & Agnostic \\
\hline Methodist & Roman Catholic & Christian Orthodox \\
\hline Lutheran & Southern Baptist & Other Baptist* \\
\hline Anglican & Presbyterian & Seventh Day Adventist \\
\hline Church of God & Church of Christ & Church of God in Christ \\
\hline Pentecostal & Assembly of God & Church of the Nazarene \\
\hline Episcopal & LDS/Mormon & Jehovah's Witness \\
\hline Other Christian & & \\
\hline Jewish & Muslim & Hindu \\
\hline Buddhist & Baha'i & Taoist \\
\hline Confucian & Jain & Shintoist \\
\hline Sikh & Zoroastrian & Chinese Religionist \\
\hline Pagan* & Shaman* & Ethnic/Tribal Religionist* \\
\hline Mandean & -Unitarian Universalist & \\
\hline \multicolumn{3}{|c|}{ Other Religious Background* } \\
\hline
\end{tabular}


13.) Describe yourself today. (check as many as apply)

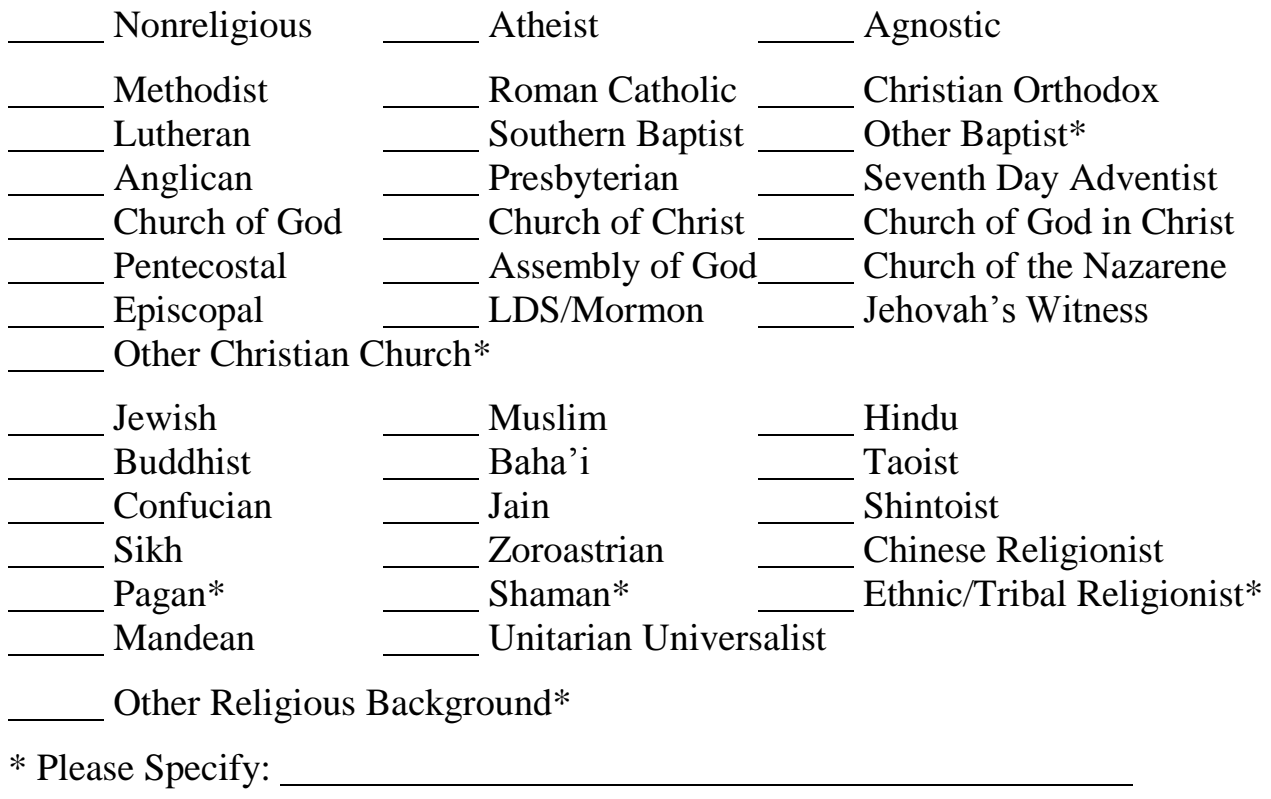

14.) Are you participating in any organized religious body now?

No $\_$Yes Where?


$\underline{\text { Appendix L }}$

Factor Analysis Data 


\section{$\underline{\text { Appendix L1 }}$}

\section{S-ROS Structure Matrix}

\begin{tabular}{|c|c|c|c|c|c|c|c|c|}
\hline Item & Components: 1 & 2 & 3 & 4 & 5 & 6 & 7 & 8 \\
\hline 17 & .766 & .137 & .097 & -.171 & .082 & -.263 & .208 & -.074 \\
\hline 36 & .755 & .398 & -.034 & -.335 & .065 & -258 & .264 & -.191 \\
\hline 23 & .753 & .021 & .079 & -.060 & -.102 & .130 & .116 & -.053 \\
\hline 7 & .694 & .271 & -.120 & -.372 & .164 & -.329 & .164 & .101 \\
\hline 19 & .634 & .041 & -.227 & -.100 & . 184 & -203 & 100 & -.008 \\
\hline 4 & .600 & .368 & .073 & -.513 & .083 & -.352 & .113 & -.099 \\
\hline 1 & .446 & -.074 & .107 & . 144 & -.367 & -.167 & .156 & .076 \\
\hline 16 & .146 & .808 & .085 & -.050 & -.118 & -.032 & .037 & -.092 \\
\hline 38 & .110 & .782 & .028 & .065 & -.148 & -.071 & .082 & -.296 \\
\hline 42 & .457 & .559 & -.079 & -.421 & -.137 & -205 & .146 & .021 \\
\hline 35 & .112 & -.392 & -.060 & -.144 & -.308 & -.304 & .085 & -.142 \\
\hline 32 & .107. & .040 & -.694 & .116. & .010 & .197 & 101 & -.052 \\
\hline 22 & -.208 & -.097 & -.678 & .034 & -.104 & .112 & -.118 & .222 \\
\hline 27 & . 126 & -.046 & .601 & -.118 & .130 & .143 & .348 & .045 \\
\hline 2 & .045 & -.170 & .065 & -.742 & .049 & -.052 & .191 & .083 \\
\hline 26 & -.142 & -.109 & .032 & .714 & -.304 & .466 & -.170 & .132 \\
\hline 25 & -.197 & .059 & .072 & .607 & -.376 & .118 & -.063 & -.099 \\
\hline
\end{tabular}

Extraction Method: Principal Component Analysis

Rotation Method: Oblimin with Kaiser Normalization

* Item is reverse-scored 
Appendix L1, Continued

\section{S-ROS Structure Matrix}

\begin{tabular}{|c|c|c|c|c|c|c|c|c|}
\hline Item & Components: 1 & 2 & 3 & 4 & 5 & 6 & 7 & 8 \\
\hline 41 & .408 & .481 & -.087 & -.551 & .052 & -.503 & .048 & .035 \\
\hline 28 & .063 & 108 & .377 & -.495 & -.164 & .291 & -.079 & -.252 \\
\hline 24 & -.151 & .002 & -.145 & .180 & -.689 & .305 & -.120 & .026 \\
\hline 33 & -.022 & 197 & -.011 & .136 & -.638 & .001 & -.303 & -.034 \\
\hline 29 & .051 & .240 & .246 & .018 & -.526 & -.009 & .302 & -.298 \\
\hline 15 & -.086 & -.010 & -.083 & .141 & -.055 & .821 & -.058 & .018 \\
\hline $11^{*}$ & 245 & .049 & .097 & -.350 & .239 & -.124 & .712 & .172 \\
\hline $31^{*}$ & .375 & .087 & -.081 & -.102 & .301 & -.228 & .622 & .011 \\
\hline 3 & .053 & .082 & .006 & .006 & -.010 & -.006 & -.026 & -.861 \\
\hline 39 & .082 & .287 & .082 & -.045 & -.259 & -.082 & 410 & -.532 \\
\hline 20 & .105 & -.039 & .101 & -.108 & .070 & -.151 & .111 & .050 \\
\hline 30 & -.106 & .063 & -.245 & .166 & -.014 & -.154 & -.042 & .059 \\
\hline 18 & -.062 & .269 & -.161 & .256 & -.118 & -.002 & -.273 & .097 \\
\hline 8 & -.024 & -.028 & .051 & .050 & .022 & .113 & .046 & -.006 \\
\hline 10 & -.003 & 197 & -.021 & .000 & -.070 & .016 & -.108 & .060 \\
\hline 21 & .420 & .301 & -.017 & -.270 & .050 & -.017 & .039 & -.342 \\
\hline$\underline{40}$ & -.101 & .220 & -.041 & -.010 & -.185 & -.086 & -.133 & -.129 \\
\hline
\end{tabular}

Extraction Method: Principal Component Analysis

Rotation Method: Oblimin with Kaiser Normalization

* Item is reverse-scored 
Appendix L1, Continued

\section{S-ROS Structure Matrix}

\begin{tabular}{|c|c|c|c|c|c|c|c|c|}
\hline Item & Components: 1 & 2 & 3 & 4 & 5 & 6 & 7 & 8 \\
\hline 34 & -.007 & .054 & .259 & .017 & -.117 & -.057 & -.135 & -.191 \\
\hline 37 & . 149 & .207 & -.269 & .188 & -.242 & .232 & -.080 & .255 \\
\hline 5 & -.015 & .365 & -.244 & .155 & -.315 & -.091 & -.295 & .002 \\
\hline 12 & -.207 & -.258 & -.142 & .064 & -.036 & .122 & -.240 & .179 \\
\hline 14 & -.055 & .289 & -.162 & .107 & -.196 & .150 & .172 & -.073 \\
\hline 9 & .199 & .234 & .012 & -.086 & -.225 & -.168 & -.021 & -.127 \\
\hline 6 & .335 & .051 & .152 & -.083 & .073 & -.165 & .133 & -.163 \\
\hline 13 & .106 & .152 & .153 & .006 & .000 & -.039 & -.351 & -.370 \\
\hline
\end{tabular}

\begin{tabular}{|c|c|c|c|c|c|c|}
\hline Item & Components: 9 & 10 & 11 & 12 & 13 & 14 \\
\hline 17 & -.090 & -.007 & -.173 & .009 & -.080 & -.305 \\
\hline 36 & -.239 & .174 & -.335 & .053 & -.218 & -.118 \\
\hline 23 & -.047 & -.027 & .121 & .135 & -.138 & -.164 \\
\hline 7 & -.246 & .232 & -.207 & .034 & -.401 & -.142 \\
\hline 38 & .023 & .051 & -.238 & .061 & -.147 & -.092 \\
\hline 42 & -.174 & .162 & -.227 & .190 & -.378 & .103 \\
\hline$\underline{35}$ & -.333 & -.184 & -.035 & .271 & -.034 & -.351 \\
\hline
\end{tabular}

Extraction Method: Principal Component Analysis

Rotation Method: Oblimin with Kaiser Normalization

* Item is reverse-scored 
Appendix L1, Continued

\section{S-ROS Structure Matrix}

\begin{tabular}{|c|c|c|c|c|c|c|c|}
\hline Item & Components: & 9 & 10 & 11 & 12 & 13 & 14 \\
\hline 32 & & .273 & -.042 & .017 & -.235 & .233 & -.041 \\
\hline 22 & & .414 & -.080 & -.082 & -.276 & .082 & .195 \\
\hline 27 & & -.051 & -.035 & -.129 & .210 & -.101 & -.227 \\
\hline $2 *$ & & -.210 & -.096 & -.109 & .110 & .062 & .010 \\
\hline 26 & & .167 & .006 & .039 & .075 & .033 & .092 \\
\hline 25 & & .198 & -.074 & -.078 & -.100 & .358 & .165 \\
\hline 41 & & -.188 & .203 & -.139 & -.001 & -.252 & -.088 \\
\hline 28 & & .057 & -.008 & -.132 & -.066 & -.115 & -.117 \\
\hline 24 & & .116 & -.234 & .032 & -.218 & .152 & .065 \\
\hline 33 & & .123 & .155 & -.057 & -.024 & .188 & -.047 \\
\hline 29 & & .287 & -.037 & -.207 & -.044 & -.289 & .006 \\
\hline 15 & & .047 & .047 & -.002 & -.037 & .179 & .161 \\
\hline $11^{*}$ & & -.222 & .245 & .118 & .023 & -.104 & -.169 \\
\hline $31 *$ & & -.389 & -.076 & .185 & .013 & -.164 & -.139 \\
\hline 3 & & -.036 & -.008 & .031 & .089 & -.079 & -.134 \\
\hline 39 & & .094 & .218 & -.526 & -.040 & -.030 & .091 \\
\hline$\underline{20}$ & & -.740 & .068 & .020 & .014 & -.002 & -.068 \\
\hline
\end{tabular}

Extraction Method: Principal Component Analysis

Rotation Method: Oblimin with Kaiser Normalization

* Item is reverse-scored 
Appendix L1, Continued

\section{S-ROS Structure Matrix}

\begin{tabular}{|c|c|c|c|c|c|c|}
\hline Item & Components: 9 & 10 & 11 & 12 & 13 & 14 \\
\hline 30 & .667 & .282 & -.157 & -.182 & .174 & .176 \\
\hline 18 & .592 & .050 & .065 & -.310 & .124 & .375 \\
\hline 8 & .053 & .879 & & -.002 & -.052 & -.117 \\
\hline 10 & .171 & .040 & -.815 & -.114 & -.065 & .063 \\
\hline 21 & -.307 & .086 & -.469 & .105 & -.068 & -.006 \\
\hline 40 & .106 & .136 & -.135 & -.761 & .169 & .174 \\
\hline 34 & .020 & .090 & -.052 & .742 & .017 & -.033 \\
\hline 37 & .392 & .012 & .105 & -.490 & .228 & .187 \\
\hline 5 & .236 & .085 & .168 & -.375 & -.294 & .240 \\
\hline 12 & -.004 & -.119 & .065 & -.111 & .716 & .095 \\
\hline 14 & .260 & -.057 & .191 & -.177 & .611 & .203 \\
\hline 9 & -.015 & .213 & .090 & .139 & -.109 & -.766 \\
\hline 6 & -.185 & .113 & -.128 & .048 & -.140 & -.748 \\
\hline$\underline{13}$ & .273 & .107 & -.049 & -298 & .140 & .556 \\
\hline
\end{tabular}

Extraction Method: Principal Component Analysis

Rotation Method: Oblimin with Kaiser Normalization

* Item is reverse-scored 


\section{Appendix L2}

\section{$\underline{\text { S-ROS Inter-Item Correlation Matrix }}$}

\begin{tabular}{|c|c|c|c|c|c|c|c|c|c|c|c|}
\hline & 1 & 2 & 3 & 4 & 5 & 6 & 7 & 8 & 9 & 10 & 11 \\
\hline 1 & 1.000 & & & & & & & & & & \\
\hline 2 & -.010 & 1.000 & & & & & & & & & \\
\hline 3 & -.036 & -.055 & 1.000 & & & & & & & & \\
\hline 4 & $.242 *$ & $.203^{*}$ & " .104 & 1.000 & & & & & & & \\
\hline 5 & .001 & $-.236^{*}$ & .039 & -.002 & 1.000 & & & & & & \\
\hline 6 & $.283^{*}$ & .051 & $.187^{+}$ & $.267^{*}$ & $-.166^{+}$ & 1.000 & & & & & \\
\hline 7 & $.302 *$ & $.205^{*}$ & -.074 & $.700^{*}$ & .068 & $.347^{*}$ & 1.000 & & & & \\
\hline 8 & $.149^{+}$ & -.114 & .025 & .012 & .008 & .124 & .021 & 1.000 & & & \\
\hline 9 & $.373^{*}$ & -.063 & $.175^{+}$ & $.261^{*}$ & .050 & $.482^{*}$ & $.239^{*}$ & .140 & 1.000 & & \\
\hline 10 & -.084 & .000 & -.078 & .007 & .037 & .038 & .127 & .025 & -.039 & 1.000 & \\
\hline 11 & .121 & $.317^{*}$ & -.091 & $.325^{*}$ & $-.173^{+}$ & $.197^{+}$ & $.323^{*}$ & $.213 *$ & .075 & -.133 & 1.000 \\
\hline 12 & $-.163^{+}$ & .061 & -.122 & $-.364^{*}$ & -.039 & $-.214^{*}$ & $-.283^{*}$ & -.060 & -.141 & -.063 & $-.185^{+}$ \\
\hline 13 & -.129 & -.018 & $.183^{+}$ & .046 & $.189^{+}$ & $-.174^{+}$ & .011 & -.028 & $-.273^{*}$ & .106 & $-.247 *$ \\
\hline 14 & -.104 & -.079 & -.013 & $-.179^{+}$ & .030 & -.137 & $-.222 *$ & -.059 & -.019 & -.029 & -.025 \\
\hline 15 & -.118 & -.058 & -.015 & $-.228 *$ & -.007 & $-.186^{+}$ & $-.223 *$ & .100 & -.142 & .018 & -.133 \\
\hline 16 & .081 & -.056 & .038 & $.270^{*}$ & $.263^{*}$ & $.154^{+}$ & $.189^{+}$ & -.034 & $.168^{+}$ & $.222 *$ & .075 \\
\hline 17 & $.278 *$ & .083 & .097 & $.445^{*}$ & -.068 & $.337 *$ & $.475^{*}$ & -.061 & $.237 *$ & . .062 & $.263^{*}$ \\
\hline
\end{tabular}

${ }^{+}$Sig. $(1-$ tailed $)<.05$

$*$ Sig. $(1$-tailed $)<.01$

Determinant $=.0000000044$ 


\section{Appendix L2, Continued}

\section{S-ROS Inter-Item Correlation Matrix}

\begin{tabular}{lcccccccccccc} 
& 1 & 2 & 3 & 4 & 5 & 6 & 7 & 8 & 9 & 10 & 11 \\
\hline 18 & $-.195^{+}$ & $-.301^{*}$ & -.083 & $-.162^{+}$ & $.392^{*}$ & $-.184^{+}$ & $-.168^{+}$ & .037 & $-.164^{+}$ & .136 & $-.281^{*}$ \\
19 & $.192^{+}$ & .038 & .045 & $.373^{*}-.095$ & $.236^{*}$ & $.500^{*}$ & $.206^{+}$ & .098 & -.015 & $.168^{+}$ \\
20 & .068 & .169 & -.030 & $.201^{*}-.131$ & $.221^{*}$ & $.148^{+}$ & -.041 & -.013 & -.121 & $.232^{*}$ \\
21 & .067 & .076 & $.176^{+}$ & $.383^{*}-.055$ & .130 & $.390^{*}$ & -.007 & .115 & $.223^{*}$ & .101 \\
22 & $-.201^{*}$ & -.006 & -.120 & $-.269^{*}$ & $.283^{*}$ & $-.217^{*}$ & -.107 & -.049 & $-.182^{+}$ & $.168^{+}$ & $-.158^{+}$ \\
23 & $.328^{*}$ & .035 & .089 & $.336^{*}-.010$ & $.201^{*}$ & $.319^{*}$ & -.032 & $.161^{+}$ & -.069 & $.207^{+}$ \\
24 & .074 & -.099 & -.035 & $-.261^{*}$ & $.231^{*}-.136$ & $-.233^{*}$ & -.103 & -.031 & .050 & $-.305^{*}$ \\
25 & .036 & $-.314^{*}$ & .023 & $-.445^{*}$ & $.155^{+}$ & -.130 & $-.395^{*}$ & .012 & -.127 & .058 & $-.271^{*}$ \\
26 & .113 & $-.425^{*}$ & -.081 & $-.427^{*}$ & .128 & -.126 & $-.313^{*}$ & .081 & -.118 & .042 & $-.340^{*}$ \\
27 & .066 & .071 & -.028 & .130 & $-.229^{*}$ & $.200^{*}$ & .050 & .070 & .036 & -.013 & $.263^{*}$ \\
28 & -.068 & $.213^{*}$ & .102 & .111 & -.023 & .122 & .019 & .069 & .095 & .049 & -.028 \\
29 & .092 & -.077 & .138 & .126 & .120 & .067 & -.001 & -.023 & $.147^{+}$ & $.162^{+}$ & .041 \\
30 & -.056 & -.139 & -.119 & $-.162^{+}$ & $.182^{+}$ & $-.162^{+}$ & -.058 & $.143^{+}$ & -.032 & $.164^{+}$ & -.129 \\
31 & .087 & .049 & .039 & $.253^{*}-.117$ & $.202^{+}$ & $.402^{*}-.091$ & .076 & $-.194^{+}$ & $.479^{*}$ \\
32 & -.054 & $-.143^{+}$ & .017 & -.106 & .087 & -.031 & -.026 & -.024 & .005 & -.018 & -.033 \\
33 & .103 & $-.196^{+}$ & .014 & -.067 & .126 & -.035 & $-.186^{+}$ & .043 & $.214^{*}$ & .114 & $-.245^{*}$ \\
34 & .020 & -.020 & $.171^{+}$ & .069 & -.089 & .030 & -.027 & .027 & $.148^{+}$ & -.022 & -.084 \\
\hline
\end{tabular}

${ }^{+}$Sig. $(1-$ tailed $)<.05$

$*$ Sig. $(1$-tailed $)<.01$

Determinant $=.0000000044$ 
Appendix L2, Continued

$\underline{\text { S-ROS Inter-Item Correlation Matrix }}$

\begin{tabular}{|c|c|c|c|c|c|c|c|c|c|c|c|}
\hline & 1 & 2 & 3 & 4 & 5 & 6 & 7 & 8 & 9 & 10 & 11 \\
\hline 35 & $.228 *$ & $.182^{+}$ & .129 & .083 & -.138 & $.254^{*}$ & .032 & -.125 & $.199^{+}$ & -.107 & .018 \\
\hline 36 & $.282 *$ & $=.127$ & . 107 & $.607 *$ & -.010 & $.342^{*}$ & .694* & -.023 & $.168^{+}$ & .131 & $.301^{*}$ \\
\hline 37 & -.006 & $-.155^{+}$ & $-.176^{+}$ & -.133 & $.244^{*}$ & $-.160^{+}$ & .010 & -.021 & -.035 & .062 & -.068 \\
\hline 38 & .023 & -.111 & $.253 *$ & $.223 *$ & $.186^{+}$ & .124 & $.156^{+}$ & .001 & $.251^{*}$ & $.182^{+}$ & -.017 \\
\hline 39 & .119 & .009 & $.312 *$ & $.161^{+}$ & .026 & .072 & .134 & .112 & .026 & $.260 *$ & .067 \\
\hline 40 & -.111 & -.100 & .026 & -.056 & $.318^{*}$ & $-.159^{+}$ & -.063 & .051 & -.029 & $.173^{+}$ & -.089 \\
\hline 41 & .036 & $.214 *$ & -.050 & $.684^{*}$ & $.152^{+}$ & $.221^{*}$ & $.559^{*}$ & .030 & $.190^{+}$ & .072 & $.251^{*}$ \\
\hline 42 & .141 & $.216^{*}$ & -.013 & $.564^{*}$ & $.166^{+}$ & .025 & $.568^{*}$ & .032 & .131 & $.209 *$ & $.212^{+}$ \\
\hline
\end{tabular}

$12 \quad 1.000$

$\begin{array}{lll}13 & -.020 & 1.000\end{array}$

$14 \quad .188^{+} \quad .157^{+} 1.000$

$\begin{array}{lllll}15 & .173^{+} & .073 & .147^{+} & 1.000\end{array}$

$\begin{array}{llllll}16 & -.201^{+} & .194^{+} & .176^{+} & .039 & 1.000\end{array}$

$\begin{array}{lllllll}17 & -.124^{+} & -.084 & -.125 & -.234^{*} & .128 & 1.000\end{array}$

$\begin{array}{llllllll}18 & .072 & .283 * & .327 * & .046 & .150^{+}-.110 & 1.000\end{array}$

${ }^{+}$Sig. $(1$-tailed $)<.05$

$*$ Sig. $(1$-tailed $)<.01$

Determinant $=.0000000044$ 


\section{Appendix L2, Continued}

\section{$\underline{\text { S-ROS Inter-Item Correlation Matrix }}$}

\begin{tabular}{lllllllllll}
12 & 13 & 14 & 15 & 16 & 17 & 18 & 19 & 20 & 21 & 22 \\
\hline
\end{tabular}

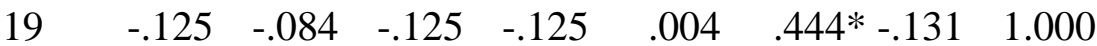

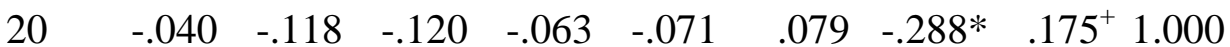

$\begin{array}{llllllllll}21 & -.188^{+} & .090 & -.014 & -.048 & .201^{+} & .272 *-.116 & .254^{*} & .163^{+} & 1.000\end{array}$

$22 \quad .170^{+}-.010 \quad .115 \quad .144^{+}-.077 \quad-.205^{*} \quad .364^{*}-.118{ }^{-}-.215^{*}-.170^{+} 1.000$

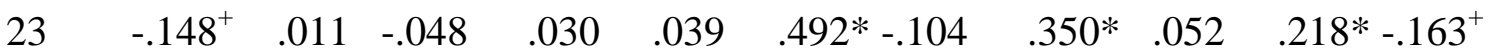

$24 \quad \begin{array}{llllllllllll}216^{*} & .016 & .237^{*} & .173^{+} & .081 & -.194^{+} & .133 & -.200^{+} & -.155^{+} & -.099 & .182^{+}\end{array}$

$25 \quad .227^{*} \quad .146^{+} \quad .196^{+} \quad .138 \quad .074 \quad-.150^{+} \quad .219^{*}-.225^{*}-.136 \quad-.158^{+} \quad .081$

$\begin{array}{lllllllllll}26 & .087 & -.044 & .092 & .374 *-.088 & -.211^{*} & .182^{+}-.173^{+}-.107 & -.244 * & .049\end{array}$

$\begin{array}{lllllllllll}27 & -.125 & -.161^{+} & -.153^{+} & .064 & .013 & .188^{+}-.151^{+}-.029 & .073 & .109 & -.314^{*}\end{array}$

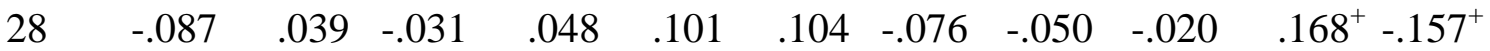

$\begin{array}{llllllllllll}29 & -.298 * & .082 & .058 & -.049 & .205^{+} & .069 & .114 & -.061 & -.127 & .047 & -.068\end{array}$

$\begin{array}{llllllllll}30 & .045 & .228^{*} & .167^{+}-.035 & .120 & -.079 & .356^{*}-.145^{+}-.32 *^{*}-.163^{+} & .342^{*}\end{array}$

$\begin{array}{lllllllllll} & -.191^{+} & -.227 * & .014 & -.162^{+} & .005 & .320^{*}-.234^{*} & .377^{*} & .250^{*} & .090 & -.189^{+}\end{array}$

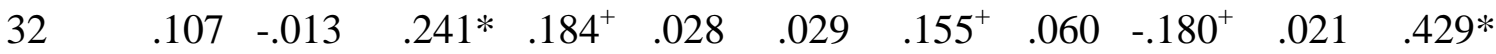

$\begin{array}{lllllllllll}33 & .002 & .091 & .136 & .075 & .130 & -.054 & .171^{+}-.114 & -.024 & .002 & .088\end{array}$

$\begin{array}{llllllllllll}34 & -.038 & -.024 & -.087 & -.040 & -.071 & .001 & -.102 & -.033 & -.019 & .056 & -.189^{+}\end{array}$

\begin{tabular}{llllllllllll}
35 & .004 & $-.233^{*}$ & -.136 & $-.183^{+}$ & $-.251^{*}$ & .124 & $-.283^{*}$ & .142 & $.172^{+}$ & .073 & -.063 \\
\hline
\end{tabular}

${ }^{+}$Sig. $(1-$ tailed $)<.05$

$*$ Sig. $(1$-tailed $)<.01$

Determinant $=.0000000044$ 


\section{Appendix L2, Continued}

$\underline{\text { S-ROS Inter-Item Correlation Matrix }}$

\begin{tabular}{lcccccccccccc} 
& 12 & 13 & 14 & 15 & 16 & 17 & 18 & 19 & 20 & 21 & 22 \\
\hline 36 & $-.319^{*}$ & .038 & -.087 & -.127 & $.311^{*}$ & $.624^{*}-.154^{+}$ & $.470^{*}$ & $.165^{+}$ & $.551^{*}-.199^{+}$ \\
37 & .132 & $.156^{+}$ & $.295^{*}$ & $.178^{+}$ & $.154^{+}$ & .013 & $.365^{*}-.030$ & $-.180^{+}$ & -.126 & $.384^{*}$ \\
38 & $-.246^{*}$ & .032 & .075 & -.075 & $.60 *^{*}$ & $.160^{+}$ & .035 & .065 & -.035 & $.26 *^{*}-.132$ \\
39 & $-.145^{+}$ & .126 & .102 & .003 & $.269^{*}$ & $.148^{+}-.025$ & -.009 & -.041 & $.308^{*}-.070$ \\
40 & .110 & $.242^{*}$ & $.171^{+}-.010$ & $.249^{*}-.012$ & $.21 *^{*}-.107$ & -.035 & .018 & $.148^{+}$ \\
41 & $-.170^{+}$ & -.014 & -.142 & $-.338^{*}$ & $.375^{*}$ & $.40 *^{*}-.019$ & $.375^{*}$ & .140 & $.26 *^{*}-.076$ \\
42 & $-.356^{*}-.020$ & -.037 & $-.146^{+}$ & $.326^{*}$ & $.324^{*}$ & .009 & $.324^{*}$ & .077 & $.380^{*}-.124$ \\
\hline
\end{tabular}

\begin{tabular}{lllllllllll}
23 & 24 & 25 & 26 & 27 & 28 & 29 & 30 & 31 & 32 & 33 \\
\hline
\end{tabular}

$23 \quad 1.000$

$\begin{array}{lll}24 & -.019 & 1.000\end{array}$

$25 \quad-.121 \quad .252 * 1.000$

$26 \quad .017 \quad .313 * \quad .402 * 1.000$

$\begin{array}{lllll}27 & .152^{+}-.137 & -.083 & -.073 & 1.000\end{array}$

$\begin{array}{lllllll}28 & .084 & .047 & -.092 & -.127 & .145^{+} & 1.000\end{array}$

$29 \quad \begin{array}{llllll}081 & .203 * & .185^{+} & .074 & .105 & .206 * 1.000\end{array}$

$\begin{array}{lllllllll}30 & -.113 & .004 & .081 & .089 & -.173^{+} & -.127 & .016 & 1.000\end{array}$

${ }^{+}$Sig. $(1$-tailed $)<.05$

* Sig. $(1$-tailed $)<.01$

Determinant $=.0000000044$ 


\section{Appendix L2, Continued}

\section{S-ROS Inter-Item Correlation Matrix}

\begin{tabular}{lcccccccccccc} 
& 23 & 24 & 25 & 26 & 27 & 28 & 29 & 30 & 31 & 32 & 33 \\
\hline 31 & $.210^{+}$ & $-.205^{+}$ & $-.250^{*}$ & $-.262^{*}$ & $.171^{+}$ & -.078 & -.083 & $-.208^{+}$ & 1.000 & & \\
32 & .061 & $.157^{+}$ & .106 & .034 & $-.267^{*}$ & $-.164^{+}$ & -.052 & $.257^{*}-.017$ & 1.000 & \\
33 & .049 & $.346^{*}$ & $.210^{*}$ & $.167^{+}$ & -.110 & .021 & $.212^{*}$ & .115 & $-.276^{*}$ & .028 & 1.000 \\
34 & .042 & -.094 & .071 & .127 & $.179^{+}$ & .074 & .070 & -.074 & -.141 & $-.234^{*}$ & .104 \\
35 & .125 & .063 & -.033 & -.111 & .076 & -.003 & .026 & $-.186^{+}$ & .107 & -.087 & .045 \\
36 & $.374 *$ & $-.270^{*}$ & $-.227^{*}$ & $-.337^{*}$ & .094 & $.166^{+}$ & .142 & -.082 & $.395^{*}$ & .063 & -.011 \\
37 & .060 & $.245^{*}$ & $.232^{*}$ & $.191^{+}$ & $-.233^{*}$ & -.043 & .084 & $.228^{*}-.133$ & $.280^{*}$ & $.191^{+}$ \\
38 & .091 & .004 & $.151^{+}$ & -.016 & -.005 & .083 & $.279^{*}$ & .051 & .033 & -.019 & .133 \\
39 & -.031 & .057 & .102 & -.056 & .081 & $.167^{+}$ & $.444^{*}$ & $.173^{+}$ & .090 & .025 & .077 \\
40 & $-.167^{+}$ & $.165^{+}$ & .116 & -.075 & -.138 & .083 & .070 & $.161^{+}-.111$ & .116 & $.171^{+}$ \\
41 & .095 & $-.244^{*}$ & $-.329^{*}$ & $-.508^{*}$ & .032 & $.154^{+}$ & .099 & -.080 & $.258^{*}$ & .000 & .038 \\
42 & $.259^{*}-.080$ & $-.224^{*}-.281^{*}$ & .033 & $.190^{+}$ & $.157^{+}-.096$ & $.233^{*}-.076$ & .031 \\
\hline
\end{tabular}

$34 \quad 35 \quad 36$

$34 \quad 1.000$

$35 \quad .101 \quad 1.000$

$\begin{array}{llll}36 & .050 \quad .076 \quad 1.000\end{array}$

${ }^{+}$Sig. $(1-$ tailed $)<.05$

$*$ Sig. $(1$-tailed $)<.01$

Determinant $=.0000000044$ 
Appendix L2, Continued

$\underline{\text { S-ROS Inter-Item Correlation Matrix }}$

\begin{tabular}{llllllllll} 
& 34 & 35 & 36 & 37 & 38 & 39 & 40 & 41 & 42 \\
\hline 37 & $-.238^{*}$ & $-.276^{*}$ & -.013 & 1.000 & & & & \\
38 & .098 & $-.156^{+}$ & $.289^{*}$ & .056 & 1.000 & & & & \\
39 & .097 & -.025 & $.341^{*}-.053$ & $.356^{*}$ & 1.000 & & \\
40 & $-.325 *$ & -.138 & .013 & $.299 *$ & .090 & $.199^{+}$ & 1.000 & \\
41 & .006 & .074 & $.594^{*}-.102$ & $.260^{*}$ & .140 & .073 & 1.000 \\
42 & .082 & .042 & $.588^{*}-.021$ & $.452^{*}$ & $.224 *-.042$ & $.589 * 1.000$ \\
\hline
\end{tabular}

${ }^{+}$Sig. $(1$-tailed $)<.05$

$*$ Sig. $(1$-tailed $)<.01$

Determinant $=.0000000044$ 


\section{$\underline{\text { Appendix L3 }}$}

\section{RITSF Structure Matrix}

\begin{tabular}{|c|c|c|c|c|c|}
\hline Item Components: & & 2 & 3 & 4 & 5 \\
\hline 2 & .804 & -.078 & .211 & .075 & .118 \\
\hline 3 & .780 & -.409 & .293 & .108 & -.021 \\
\hline 11 & .779 & -.190 & .399 & .082 & .142 \\
\hline 1 & .766 & -.341 & .332 & .081 & -.137 \\
\hline 19 & .691 & .070 & .266 & 183 & -.370 \\
\hline 6 & .509 & -.479 & .472 & -.207 & .025 \\
\hline 12 & .499 & 199 & .469 & .032 & -.461 \\
\hline 5 & .207 & -.735 & .215 & .101 & -.152 \\
\hline 4 & .456 & -.609 & .323 & .175 & .134 \\
\hline 7 & -.018 & .563 & .238 & .217 & .003 \\
\hline 8 & .241 & -.128 & .723 & .185 & -.115 \\
\hline 13 & .161 & . 143 & .706 & .339 & -.387 \\
\hline 15 & .378 & -.095 & .673 & .110 & -.139 \\
\hline 14 & .372 & .120 & .664 & .286 & -.032 \\
\hline 9 & .359 & .209 & .583 & .298 & .419 \\
\hline 10 & .077 & .464 & .477 & .110 & -.161 \\
\hline 17 & .004 & -.051 & .108 & .806 & -.025 \\
\hline 16 & .382 & .105 & .363 & .718 & -.175 \\
\hline$\underline{18}$ & .209 & -.102 & .388 & .292 & -.724 \\
\hline
\end{tabular}

Extraction Method: Principal Component Analysis

Rotation Method: Oblimin with Kaiser Normalization 


\section{Appendix L4}

$\underline{\text { RITSF Inter-Item Correlation Matrix }}$

\begin{tabular}{|c|c|c|c|c|c|c|c|c|c|c|c|}
\hline & 1 & 2 & 3 & 4 & 5 & 6 & 7 & 8 & 9 & 10 & 11 \\
\hline 1 & 1.000 & & & & & & & & & & \\
\hline 2 & $.527^{*}$ & 1.000 & & & & & & & & & \\
\hline 3 & $.587^{*}$ & $.540 *$ & 1.000 & & & & & & & & \\
\hline 4 & $.465^{*}$ & $.331 *$ & $.515^{*}$ & 1.000 & & & & & & & \\
\hline 5 & $.307^{*}$ & .095 & $.360 *$ & $.467 *$ & 1.000 & & & & & & \\
\hline 6 & $.526^{*}$ & $.292 *$ & $.441 *$ & $.364 *$ & $.381^{*}$ & 1.000 & & & & & \\
\hline 7 & .005 & -.031 & -.131 & -.129 & $-.189^{+}$ & $-.186^{+}$ & 1.000 & & & & \\
\hline 8 & $.236^{*}$ & $.216^{*}$ & $.248 *$ & $.280 *$ & .134 & $.320 *$ & .054 & 1.000 & & & \\
\hline 9 & $.164^{+}$ & $.322 *$ & $.219 *$ & .132 & -.003 & $.207^{*}$ & $.204^{*}$ & $.363^{*}$ & 1.000 & & \\
\hline 10 & .021 & -.027 & -.005 & -.088 & -.132 & .038 & $.240 *$ & $.223^{*}$ & $.253^{*}$ & 1.000 & \\
\hline 11 & $.572^{*}$ & $.495^{*}$ & $.636^{*}$ & $.369 *$ & $.170^{+}$ & $.472 *$ & -.066 & $.253^{*}$ & $.361^{*}$ & .072 & 1.000 \\
\hline 12 & $.335^{*}$ & $.294 *$ & $.282 *$ & $.153^{+}$ & .075 & $.198^{*}$ & .136 & $.242^{*}$ & $.226^{*}$ & $.288^{*}$ & $.347^{*}$ \\
\hline 13 & $.211^{*}$ & -.013 & $.147^{+}$ & $.164^{+}$ & .067 & $.170^{+}$ & $.248^{*}$ & $.388^{*}$ & $.236^{*}$ & $.329 *$ & $.183^{+}$ \\
\hline 14 & $.172^{+}$ & $.301^{*}$ & $.223 *$ & $.183^{+}$ & .104 & $.266^{*}$ & $.150^{+}$ & $.316^{*}$ & $.378^{*}$ & $.210^{*}$ & $.315^{*}$ \\
\hline 15 & $.355^{*}$ & $.274 *$ & $.267 *$ & $.252^{*}$ & $.206^{*}$ & $.305^{*}$ & .077 & $.401 *$ & $.284 *$ & $.204 *$ & $.328 *$ \\
\hline 16 & $.219^{*}$ & $.248 *$ & $.245^{*}$ & $.219^{*}$ & .052 & .039 & .104 & $.280^{*}$ & $.317^{*}$ & $.148^{+}$ & $.271^{*}$ \\
\hline 17 & .101 & -.020 & .043 & .070 & .110 & -.048 & .075 & .091 & .132 & .132 & .059 \\
\hline
\end{tabular}

${ }^{+}$Sig. $(1$-tailed $)<.05$

$*$ Sig. $(1$-tailed $)<.01$

Determinant $=.001256$ 
Appendix L4, Continued

$\underline{\text { RITSF Inter-Item Correlation Matrix }}$

\begin{tabular}{|c|c|c|c|c|c|c|c|c|c|}
\hline & 1 & 2 & 3 & 4 & 5 & 6 & 7 & 8 & 9 \\
\hline 18 & $.237 *$ & .127 & $.214 *$ & .051 & $.211^{*}$ & .133 & .012 & $.400 *$ & .057 \\
\hline \multirow[t]{2}{*}{19} & $.445 *$ & $.450 *$ & $.429 *$ & $.163^{+}$ & $.141^{+}$ & $.286^{*}$ & .059 & $.168^{+}$ & $.147^{+}$ \\
\hline & 12 & 13 & 14 & 15 & 16 & 17 & 18 & 19 & \\
\hline 12 & 1.000 & & & & & & & & \\
\hline 13 & $.417 *$ & 1.000 & & & & & & & \\
\hline 14 & $.315^{*}$ & $.453^{*}$ & 1.000 & & & & & & \\
\hline 15 & $.255^{*}$ & $.374 *$ & $.433^{*}$ & 1.000 & & & & & \\
\hline 16 & $.242 *$ & $.363^{*}$ & $.394 *$ & $.219^{*}$ & 1.000 & & & & \\
\hline 17 & .006 & $.192^{+}$ & .095 & .079 & $.286^{*}$ & 1.000 & & & \\
\hline 18 & $.330 *$ & $.346^{*}$ & $.225 *$ & $.279 *$ & $.334 *$ & $.141^{+}$ & 1.000 & & \\
\hline 19 & $.356 *$ & $.214 *$ & $.306^{*}$ & $.327 *$ & $.358^{*}$ & .078 & $.261^{*}$ & 1.000 & \\
\hline
\end{tabular}

${ }^{+}$Sig. $(1-$ tailed $)<.05$

$*$ Sig. $(1$-tailed $)<.01$

Determinant $=.001256$ 


\section{$\underline{\text { Appendix L5 }}$}

STPE Structure Matrix

\begin{tabular}{|c|c|c|c|c|c|c|}
\hline Item & Components: 1 & 2 & 3 & 4 & 5 & 6 \\
\hline 10 & .755 & -.003 & -.018 & -.236 & -.045 & -.062 \\
\hline 11 & .735 & .111 & .105 & -.307 & .077 & .047 \\
\hline 15 & .647 & -.002 & .150 & .055 & -.284 & -.157 \\
\hline 17 & .623 & -.011 & .042 & .149 & .046 & -.481 \\
\hline 14 & .554 & -.135 & -.051 & .101 & -.207 & -.369 \\
\hline 18 & .497 & -.096 & -.112 & .120 & .147 & .139 \\
\hline 16 & . 406. & -.155 & -.287 & -.088 & .393 & -.373 \\
\hline 5 & -.002 & -.851 & -.101 & -.044 & -.119 & -.134 \\
\hline 4 & -.057 & -.809 & -.036 & .035 & -.140 & -.084 \\
\hline 6 & .137 & -.798 & .016 & .162 & -.382 & .022 \\
\hline 8 & .040 & -.005 & .950 & -.105 & -.122 & -.027 \\
\hline 9 & .008 & .120 & .943 & -.109 & -.108 & -.042 \\
\hline 12 & .031 & .186 & .036 & -.753 & -.181 & .274 \\
\hline 3 & .092 & -.250 & .112 & -.683 & -.012 & -.299 \\
\hline 7 & .013 & -.193 & -.281 & .440 & .236 & .078 \\
\hline 1 & .139 & -.253 & .149 & -.221 & -.833 & -.092 \\
\hline 2 & .027 & -.303 & .042 & -.086 & -.824 & -.125 \\
\hline$\underline{13}$ & .011 & -.078 & .057 & -.047 & -.095 & -.805 \\
\hline
\end{tabular}

Extraction Method: Principal Component Analysis

Rotation Method: Oblimin with Kaiser Normalization 


\section{Appendix L6}

\section{STPE Inter-Item Correlation Matrix}

\begin{tabular}{|c|c|c|c|c|c|c|c|c|c|c|c|}
\hline Items & 1 & 2 & 3 & 4 & 5 & 6 & 7 & 8 & 9 & 10 & 11 \\
\hline 1 & 1.000 & & & & & & & & & & \\
\hline 2 & $.653 *$ & 1.000 & & & & & & & & & \\
\hline 3 & $.219^{*}$ & .084 & 1.000 & & & & & & & & \\
\hline 4 & $.226^{*}$ & $.257 *$ & .095 & 1.000 & & & & & & & \\
\hline 5 & $.203^{+}$ & $.269 *$ & $.224 *$ & $.527 *$ & 1.000 & & & & & & \\
\hline 6 & $.363 *$ & $.382 *$ & .063 & $.557^{*}$ & $.608 *$ & 1.000 & & & & & \\
\hline 7 & $-.182^{+}$ & -.067 & -.110 & .073 & .111 & .028 & 1.000 & & & & \\
\hline 8 & .096 & .036 & .063 & -.034 & -.108 & -.063 & $-.234 *$ & 1.000 & & & \\
\hline 9 & .136 & .058 & .137 & .018 & -.018 & .083 & $-.163^{+}$ & $.849^{*}$ & 1.000 & & \\
\hline 10 & .130 & .022 & .136 & -.041 & .017 & .061 & -.046 & .027 & -.018 & 1.000 & \\
\hline 11 & .062 & -.046 & $.150^{+}$ & $-.166^{+}$ & -.092 & -.027 & -.055 & .072 & .149 & .606 & 1.000 \\
\hline 12 & $.156^{+}$ & .129 & $.229 *$ & -.071 & -.090 & $-.157^{+}$ & $-.207^{*}$ & .056 & .046 & .130 & $.210^{*}$ \\
\hline 13 & .099 & .106 & $.162^{+}$ & .102 & .112 & .003 & .000 & .043 & .038 & .074 & .015 \\
\hline 14 & $.147^{+}$ & $.166^{+}$ & .064 & .078 & .096 & $.191^{+}$ & -.060 & -.011 & -.011 & $.312^{*}$ & $.224^{*}$ \\
\hline 15 & $.233^{*}$ & .105 & .080 & -.045 & .019 & $.157^{+}$ & -.010 & .117 & .125 & $.448^{*}$ & $.308^{*}$ \\
\hline$\underline{16}$ & $-.141^{+}$ & -.098 & .058 & .075 & .082 & -.044 & -.015 & -.139 & -.180 & $.206^{*}$ & $.179^{+}$ \\
\hline
\end{tabular}

${ }^{+}$Sig. $(1$-tailed $)<.05$

$*$ Sig. $(1$-tailed $)<.01$

Determinant $=.003563$ 
Appendix L6, Continued

$\underline{\text { STPE Inter-Item Correlation Matrix }}$

\begin{tabular}{lllllllllllll} 
Items & 1 & 2 & 3 & 4 & 5 & 6 & 7 & 8 & 9 & 10 & 11 \\
\hline 17 & .048 & .037 & .068 & -.041 & -.026 & .039 & .032 & .030 & .089 & $.296^{*}$ & $.339^{*}$ \\
18 & .037 & -.044 & .016 & .026 & -.031 & .039 & .033 & -.075 & -.062 & $.176^{+}$ & $.265^{*}$ \\
\hline
\end{tabular}

$\begin{array}{llllllll}\text { Items } & 12 & 13 & 14 & 15 & 16 & 17 & 18\end{array}$

$12 \quad 1.000$

$\begin{array}{lll}13 & -.135 & 1.000\end{array}$

$\begin{array}{llll}14 & -.061 & .083 & 1.000\end{array}$

$15 \quad .030 \quad .121 \quad .335 * 1.000$

$\begin{array}{llllll}16 & -.032 & .069 & .251^{*} & .078 & 1.000\end{array}$

$17 \quad \begin{array}{llllll}.136 & .167^{+} & .372 * & .318^{*} & .340^{*} & 1.000\end{array}$

$\begin{array}{lllllll}18 & -.080 & -.030 & .095 & .192^{+} & .192^{+} & .208 * 1.000\end{array}$

${ }^{+}$Sig. $(1$-tailed $)<.05$

$*$ Sig. $(1$-tailed $)<.01$

Determinant $=.003563$ 


\section{Appendix M:}

\section{Regression Diagnostics}

Before conducting the primary analyses of interest, regression diagnostics were conducted to screen the data to determine whether they met the assumptions of these primary analyses. In regression analyses, several assumptions must be met in order for the mathematical calculations inherent in the analyses to yield meaningful results. The variables must often be transformed, the data must be complete, the variables must not be perfectly multicollinear, the data must have been measured correctly, the independent variables should contribute to reducing error variance in the prediction of the dependent variables, the data must be normally distributed, the variables must be linearly related, the variances of the error terms must be constant, and there must be no outliers in multivariate space (Berry \& Feldman, 1985). Each of these assumptions was examined during data screening.

\section{Variable Transformations}

The categorical independent variable faith type was effects coded to make it appropriate for use in regression. Effects coding was chosen in preference to dummy coding, because the goal was to contrast religiousness and spirituality against one another (rather than a control group, as in dummy coding) (Aiken \& West, 1991). All participants who received a religious vignette were assigned a value of 1 , while all participants who received a spiritual vignette were assigned a value of -1 . The variable faith magnitude was coded as a continuous variable by using the magnitude estimation scaling values from Table 1 for each vignette version (Jaccard \& Turrisi, 2003). The continuous 
independent variables in the current study were standardized (or $z$-scored) by subtracting the mean from each variable and then dividing the result by that variable's standard deviation, in order to form a new scale for each variable. For example, the mean of the MCKAS-A scores was 5.72 and the standard deviation was 0.60 , so 5.72 was subtracted from each participant's MCKAS-A score and divided by 0.60 to yield standardized MCKAS-A scores with a mean of 0 and a standard deviation of 1 . The S-ROS-I $\mathrm{I}_{\mathrm{R}}, \mathrm{S}-$ ROS-I $\mathrm{I}_{S}$, and RITSF-Help subscales and the faith magnitude values from the religious and spiritual vignettes were also standardized in the same way. Standardizing these variables was done for three reasons: (a) to avoid problems with multicollinearity between variables and their interaction terms (Aiken \& West, 1991; Jaccard \& Turrisi, 1990), (b) to increase the interpretability of interaction terms (Aiken \& West, 1991; Judd \& McLelland, 1989), and (c) to avoid substantively meaningless interpretations of regression coefficients when using scales which do not have 0 as a possible or meaningful value, such as the MCKAS-A, MCKAS-K, S-ROS- $\mathrm{I}_{\mathrm{R}}, \mathrm{S}-\mathrm{ROS}-\mathrm{I}_{\mathrm{S}}$, and RITSFHelp (Jaccard \& Turrisi, 2003). A basic frequency analysis was also conducted in SPSS before standardizing the data, to ensure the data were entered with all expected values for the scales. The frequency analysis demonstrated that the data were in fact entered with all expected values.

\section{Missing Data}

The full data set $(N=145)$ was examined for missing responses. Four surveys were returned with substantial (i.e., > 20\%) missing responses and were eliminated from the data set, resulting in a final sample size of $N=141$. Within this data set, $3.92 \%$ of STPE responses, $1.57 \%$ of MCKAS responses, $1.61 \%$ of RITSF responses, and $6.97 \%$ of 
S-ROS responses were missing. On the STPE, MCKAS, and RITSF, responses were missing in a random pattern, with the exception of five participants who omitted large numbers of responses on one of these scales, whose scales with large numbers of missing responses were not scored. On the S-ROS, responses were missing mostly from items contributing to the S-ROS-I $I_{R}$ - especially the items developed for the ROS-R-Ep and ROS-R-Es subscales. Missing data on the S-ROS, however, are coded the same as N/A responses; they do not pose a problem as long as the majority of items are not coded in this way. On the STPE, no response on item 18 was coded as $1=$ Not Very Likely if there were no other missing responses for this participant on the STPE, because it was judged that this participant intended this response by leaving the item blank. Two participants also gave one item from items $10-18$ a rating of 7 while leaving the remaining items 10 -18 blank, so their missing responses on those items were similarly coded as $1=$ Not Very Likely. To check for reliability, a second graduate student looked over the surveys and independently came to the same conclusions. This lowered the amount of missing data on the STPE to $2.54 \%$. Missing data on the STPE, MCKAS, and RITSF were handled using pairwise deletion to use all available data and maximize the sample size (and the power of the tests conducted). The scoring of the S-ROS allows for missing data. Such a small amount of missing data was not anticipated to pose significant problems for the proposed analyses (Berry \& Feldman, 1985).

\section{Multicollinearity}

Multicollinearity refers to a high correlation between two variables in a regression equation, signifying a strong linear relationship between the two variables (Berry, 1993; Fox, 1991). Perfect multicollinearity invalidates a regression by preventing coefficients in 
the equation from being uniquely defined, violating a mathematical assumption of regression. There are two kinds of multicollinearity: univariate collinearity between two variables and multivariate collinearity between a variable and the combination of the remaining variables (Tabachnick \& Fidell, 2001). The former can be checked by inspecting the bivariate correlations among all the variables to ensure none are above 0.9 (Fox, 1991), while the latter can be checked by regressing each variable on the remaining variables and checking to make sure no Tolerance statistic is less than 0.20 , no VIF (Variance Inflation Factor) exceeds 4.0, and no Condition Index exceeds 30 (or, if it does, no single variable accounts for greater than $50 \%$ of the proportion of variance in this regression) (Belsley, Kuh, \& Welsch, 1980; Tabachnick \& Fidell, 2001). The bivariate correlations among all the variables are displayed in Table 3 in Chapter 4. No correlation exceeds 0.65 , with all but one correlation less than .50 . The highest correlation was between SROS-I $\mathrm{I}_{\mathrm{R}}$ and SROS-I $\mathrm{I}_{\mathrm{S}}$ scales $(r=.64)$. Tolerances, VIF's, and Condition Indexes for each variable regressed on the remaining variables are displayed in Table 14. No Tolerances were less than 0.48 , no Condition Index exceeded 3, and no VIF exceeded 2.1. Univariate or multivariate multicollinearity did not appear to be a problem for the current set of variables. This is not surprising, as standardizing variables greatly reduces their multicollinearity (Jaccard \& Turrisi, 1990).

\section{Measurement Error}

To control for measurement errors, a great deal of attention was given prior to the study to ensure reliable and valid instruments were selected to measure the variables of interest. As explored in detail in Chapter 3, the instruments used to measure the variables 
Table 14

Tolerance, Variance Inflation Factor (VIF), and Condition Index (CIndex) for Each Variable Regressed on Remaining Variables

\begin{tabular}{|c|c|c|c|c|c|c|}
\hline Dependent & Variable Tol & erance & VIF & Dimension & Eigenvalue & CIndex \\
\hline \multirow[t]{7}{*}{ Prognosis } & Diagnosis & .98 & 1.03 & 1 & 2.13 & 1.00 \\
\hline & Awareness & .93 & 1.08 & 2 & 1.18 & 1.34 \\
\hline & Knowledge & .84 & 1.20 & 3 & 1.00 & 1.46 \\
\hline & Religiousness & .58 & 1.74 & 4 & .97 & 1.48 \\
\hline & Spirituality & .50 & 2.02 & 5 & .78 & 1.65 \\
\hline & Helpfulness & .79 & 1.27 & 6 & .61 & 1.87 \\
\hline & & & & 7 & .33 & 2.55 \\
\hline \multirow[t]{7}{*}{ Diagnosis } & Awareness & .92 & 1.08 & 1 & 2.14 & 1.00 \\
\hline & Knowledge & .82 & 1.22 & 2 & 1.29 & 1.29 \\
\hline & Religiousness & .56 & 1.78 & 3 & 1.00 & 1.46 \\
\hline & Spirituality & .49 & 1.05 & 4 & .88 & 1.56 \\
\hline & Helpfulness & .79 & 1.27 & 5 & .78 & 1.65 \\
\hline & Diagnosis & .91 & 1.09 & 6 & .59 & 1.90 \\
\hline & & & & 7 & .32 & 2.59 \\
\hline
\end{tabular}


Table 14, continued

Tolerance, Variance Inflation Factor (VIF), and Condition Index (CIndex) for Each Variable Regressed on Remaining Variables

\begin{tabular}{|c|c|c|c|c|c|c|}
\hline Dependent & Variable Tol & erance & VIF & Dimension & Eigenvalue & CIndex \\
\hline \multirow[t]{7}{*}{ Awareness } & Knowledge & .87 & 1.15 & 1 & 2.16 & 1.00 \\
\hline & Religiousness & .56 & 1.78 & 2 & 1.14 & 1.38 \\
\hline & Spirituality & .49 & 2.04 & 3 & 1.00 & 1.47 \\
\hline & Helpfulness & .79 & 1.27 & 4 & .95 & 1.51 \\
\hline & Diagnosis & .92 & 1.09 & 5 & .81 & 1.64 \\
\hline & Prognosis & .97 & 1.03 & 6 & .63 & 1.86 \\
\hline & & & & 7 & .32 & 2.60 \\
\hline \multirow[t]{7}{*}{ Knowledge } & Religiousness & .56 & 1.78 & 1 & 2.02 & 1.00 \\
\hline & Spirituality & .52 & 1.92 & 2 & 1.18 & 1.31 \\
\hline & Helpfulness & .78 & 1.28 & 3 & 1.00 & 1.42 \\
\hline & Diagnosis & .93 & 1.08 & 4 & .95 & 1.46 \\
\hline & Prognosis & .97 & 1.03 & 5 & .87 & 1.53 \\
\hline & Awareness & .98 & 1.03 & 6 & .65 & 1.77 \\
\hline & & & & 7 & .33 & 2.47 \\
\hline
\end{tabular}


Table 14, continued

Tolerance, Variance Inflation Factor (VIF), and Condition Index (CIndex) for Each Variable Regressed on Remaining Variables

Dependent Variable Tolerance VIF Dimension Eigenvalue CIndex

\begin{tabular}{|c|c|c|c|c|c|c|}
\hline Religiousness & Spirituality & .73 & 1.37 & 1 & 1.82 & 1.00 \\
\hline & Helpfulness & .80 & 1.25 & 2 & 1.11 & 1.28 \\
\hline & Diagnosis & .94 & 1.07 & 3 & 1.00 & 1.35 \\
\hline & Prognosis & .97 & 1.03 & 4 & .95 & 1.39 \\
\hline & Awareness & .93 & 1.08 & 5 & .86 & 1.46 \\
\hline & Knowledge & .82 & 1.22 & 6 & .78 & 1.53 \\
\hline & & & & 7 & .49 & 2.94 \\
\hline Spirituality & Helpfulness & .84 & 1.20 & 1 & 1.62 & 1.00 \\
\hline & Diagnosis & .93 & 1.08 & 2 & 1.22 & 1.16 \\
\hline & Prognosis & .98 & 1.03 & 3 & 1.00 & 1.27 \\
\hline & Awareness & .93 & 1.08 & 4 & .97 & 1.30 \\
\hline & Knowledge & .88 & 1.14 & 5 & .86 & 1.37 \\
\hline & Religiousness & .84 & 1.19 & 6 & .77 & 1.46 \\
\hline & & & & 7 & .57 & 1.69 \\
\hline
\end{tabular}


Table 14, continued

Tolerance, Variance Inflation Factor (VIF), and Condition Index (CIndex) for Each Variable Regressed on Remaining Variables

\begin{tabular}{|c|c|c|c|c|c|c|}
\hline Dependent & Variable Tol & erance & VIF & Dimension & Eigenvalue & CIndex \\
\hline \multirow[t]{7}{*}{ Helpfulness } & Diagnosis & .92 & 1.09 & 1 & 1.87 & 1.00 \\
\hline & Prognosis & .98 & 1.02 & 2 & 1.29 & 1.20 \\
\hline & Awareness & .93 & 1.08 & 3 & 1.00 & 1.37 \\
\hline & Knowledge & .82 & 1.22 & 4 & .97 & 1.39 \\
\hline & Religiousness & .57 & 1.75 & 5 & .87 & 1.47 \\
\hline & Spirituality & .52 & 1.93 & 6 & .68 & 1.66 \\
\hline & & & & 7 & .32 & 2.41 \\
\hline
\end{tabular}

of interest here have suitable evidence for their reliability and validity, leading to their inclusion in the current study.

\section{Specification Error}

Specification error refers to a misspecification of the regression equation used in a regression analysis (Berry, 1993). According to Berry (1993), accurate specification of the regression equation depends on ensuring the variables which are included in the regression equation are based in theory. The theory motivating a regression must be sound in order to avoid specification error. The theoretical reasons for including the variables under investigation here were expounded upon in detail in Chapters 1 and 2 each variable was included for sound theoretical reasons. Beyond having a well developed theory, Berry and Feldman (1985) suggested testing for specification error by 
examining adjusted explained variance: "if this does not decrease when the variable in question is removed from the equation, it is clearly not playing any role in reducing the error variance and would therefore seem to be irrelevant" (p. 26). Adjusted $R^{2}$ values are displayed in Table 15 for each planned regression and for these regressions with individual variables deleted. The adjusted $R^{2}$ values suggest that although each variable was included for sound theoretical reasons, in the current data set, some variables do not contribute to the regressions being performed.

\section{Normality}

Normality refers to a particular distribution of scores for a variable - the normal distribution - in which the most frequently occurring scores cluster around the mean, and scores which are increasingly deviant from the mean occur with exponentially decreasing frequency (Spence, Cotton, Underwood, \& Duncan, 1992). Regression assumes all variables are normally distributed. The normality of a variable can be assessed via a visual inspection of the variable's frequency histogram, as well as by examining the variable's skewness and kurtosis. The skewness and kurtosis of each variable are displayed in Table 16, while the frequency histograms for each variable are displayed in Figure 3 . These data suggest each variable included in the current investigation is roughly normally distributed. Non-normality did not appear to be a problem for the current set of variables.

\section{Nonlinearity}

Nonlinearity refers to a relationship between two variables which cannot be graphically depicted using a straight line (Tabachnick \& Fidell, 2001). Nonlinearity can be detected by a visual inspection of bivariate scatterplots. Scatterplots of the bivariate 
Table 15

Adjusted $R^{2}$ Values for Planned Regressions and Regressions with Variables Deleted

Equation Adjusted $R^{2}$ $R^{2}$ Change Contributes?

Regression 1 (Attribute $=$ Awareness $) \quad$ Prognosis: -0.00

Diagnosis: -0.03

Variable Deleted:

Magnitude

Prognosis: -0.01

$-0.01$

Y

Diagnosis: $-0.03 \quad 0.00$

Type

Prognosis: -0.01

Diagnosis: $-0.03 \quad 0.00$

Magnitude*Type

Prognosis: $0.00 \quad+0.00$

Diagnosis: $-0.03 \quad 0.00$

Awareness

Prognosis: $-0.01 \quad-0.01$

Diagnosis: $-0.03 \quad 0.00$

Awareness*Magnitude

Prognosis: $0.00 \quad+0.00$

Diagnosis: $-0.03 \quad 0.00$

Awareness*Type

Prognosis: $0.01 \quad+0.01$

Diagnosis: $-0.02 \quad+0.01$

Awareness*Magnitude*Type

Prognosis: $0.00 \quad+0.00$

Diagnosis: $-0.04 \quad-0.01$

Y

$\mathrm{Y}=R^{2}$ Change $\geq 0.01$ decrease 
Table 15, Continued

Adjusted $R^{2}$ Values for Planned Regressions and Regressions with Variables Deleted Equation Adjusted $R^{2} \quad R^{2}$ Change Contributes?

Regression 2 (Attribute $=$ Knowledge) Prognosis: 0.04

Diagnosis: -0.02

Variable Deleted:

Magnitude

Type

Magnitude*Type

Knowledge

Knowledge*Magnitude

Knowledge*Type

Knowledge*Magnitude*Type
Prognosis: 0.03

Diagnosis: -0.01

Prognosis: 0.03

Diagnosis: -0.02

Prognosis: 0.04

Diagnosis: -0.01

Prognosis: 0.01

Diagnosis: -0.02

Prognosis: 0.04

Diagnosis: -0.01

Prognosis: 0.02

Diagnosis: -0.01

Prognosis: 0.04

Diagnosis: -0.03
$-0.01$

$+0.01$

$-0.01$

0.000

0.00

$+0.01$

$-0.03$

Y

0.00

0.00

$+0.01$

$-0.02$

Y

$+0.01$

0.00

$-0.01$

$\mathrm{Y}=R^{2}$ Change $\geq 0.01$ decrease 
Table 15, Continued

Adjusted $R^{2}$ Values for Planned Regressions and Regressions with Variables Deleted Equation Adjusted $R^{2}$ $R^{2}$ Change Contributes?

Regression 3 (Attribute $=$ Religiousness) Prognosis: -0.01

Diagnosis: -0.02

Variable Deleted:

Magnitude

Type

Magnitude*Type

Religiousness

Religiousness*Magnitude

Religiousness*Type

Religiousness*Magnitude*Type
Prognosis: $-0.00 \quad+0.01$

Diagnosis: $-0.01 \quad+0.01$

Prognosis: $-0.00 \quad+0.01$

Diagnosis: $-0.02 \quad 0.00$

Prognosis: $-0.01 \quad 0.00$

Diagnosis: $-0.01 \quad+0.01$

Prognosis: $0.00 \quad+0.01$

Diagnosis: $-0.02 \quad 0.00$

Prognosis: $-0.02 \quad-0.01 \quad Y$

Diagnosis: $-0.04 \quad-0.02 \quad \mathrm{Y}$

Prognosis: $-0.02 \quad-0.01 \quad$ Y

Diagnosis: $-0.01 \quad+0.01$

Prognosis: $0.00 \quad+0.01$

Diagnosis: $-0.01+0.01$

$\mathrm{Y}=R^{2}$ Change $\geq 0.01$ decrease 
Table 15, Continued

Adjusted $R^{2}$ Values for Planned Regressions and Regressions with Variables Deleted Equation Adjusted $R^{2}$ $R^{2}$ Change Contributes?

Regression 4 (Attribute $=$ Spirituality) $\quad$ Prognosis: 0.09

Diagnosis: -0.03

Variable Deleted:

Magnitude

Type

Magnitude*Type

Spirituality

Spirituality*Magnitude

Spirituality*Type

Spirituality*Magnitude*Type
Prognosis: 0.08

Diagnosis: -0.02

Prognosis: 0.08

Diagnosis: -0.03

Prognosis: 0.08

Diagnosis: -0.02

Prognosis: 0.05

Diagnosis: -0.03

Prognosis: 0.00

Diagnosis: -0.02

Prognosis: 0.08

Diagnosis: -0.02

Prognosis: 0.09

Diagnosis: -0.03
$-0.01$

Y

$+0.01$

$-0.01$

Y

0.00

$-0.01$

Y

$+0.01$

$-0.04$

Y

0.00

$-0.09$

Y

$+0.01$

$-0.01$

Y

$\begin{array}{llc} & \text { Diagnosis: }-0.02 & +0.01 \\ \text { Spirituality*Magnitude*Type } & \text { Prognosis: } 0.09 & 0.00 \\ & \text { Diagnosis: }-0.03 & 0.00 \\ \text { Change } \geq 0.01 \text { decrease } & & \end{array}$

$\mathrm{Y}=R^{2}$ Change $\geq 0.01$ decrease 
Table 15, Continued

Adjusted $R^{2}$ Values for Planned Regressions and Regressions with Variables Deleted Equation Adjusted $R^{2}$ $R^{2}$ Change Contributes?

Regression 5 (Attribute $=$ Helpfulness) Prognosis: -0.01

Diagnosis: -0.00

Variable Deleted:

Magnitude

Type

Magnitude*Type

Helpfulness

Helpfulness*Magnitude

Helpfulness*Type

Helpfulness*Magnitude*Type
Prognosis: -0.02

Diagnosis: 0.01

Prognosis: -0.01

Diagnosis: -0.01

Prognosis: -0.01

Diagnosis: 0.00

Prognosis: -0.03

Diagnosis: -0.00

Prognosis: -0.01

Diagnosis: -0.01

Prognosis: -0.01

Diagnosis: 0.01

Prognosis: -0.01

Diagnosis: 0.00
$-0.001$

Y

$+0.01$

0.00

$-0.01$

Y

0.00

$+0.00$

$-0.02$

Y

0.00

0.00

$-0.01$

Y

0.00

$+0.01$

0.00

$+0.00$

$\mathrm{Y}=R^{2}$ Change $\geq 0.01$ decrease 
Table 16

Skewness and Kurtosis of Each Variable

$\underline{\text { Scale }}$

Prognosis

Diagnosis

Multicultural Awareness

Multicultural Knowledge

Religiousness

Spirituality

View of Faith Helpfulness Skewness

Kurtosis

0.56

2.84

$-1.53 \quad 2.84$

$-0.40$

0.44

0.18

$-0.73$

$-0.40$

$-0.54$

$-0.05$ $-0.62$ 


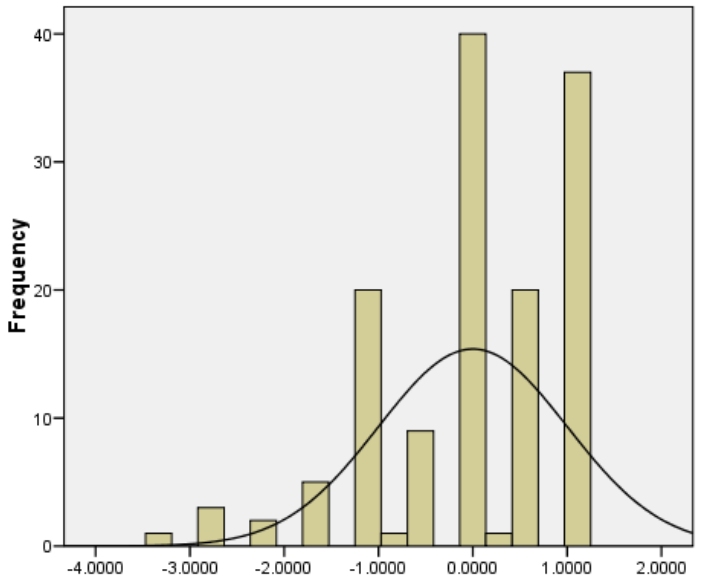

Prognosis

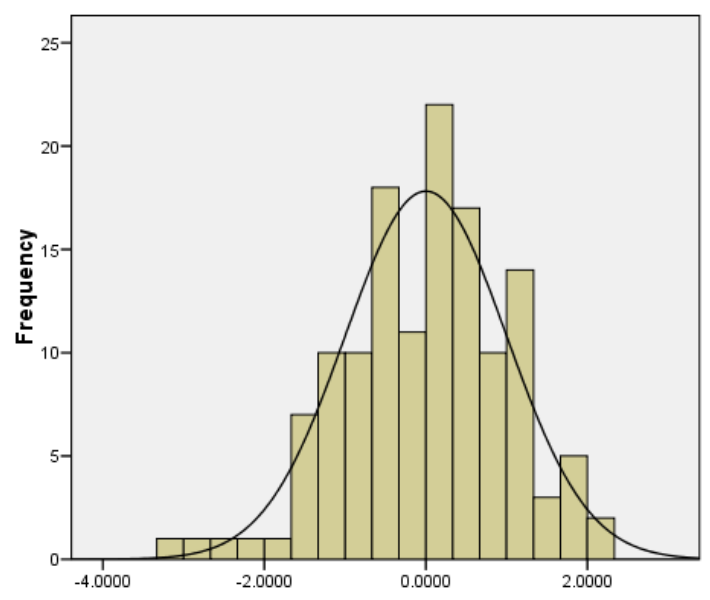

Multicultural Awareness

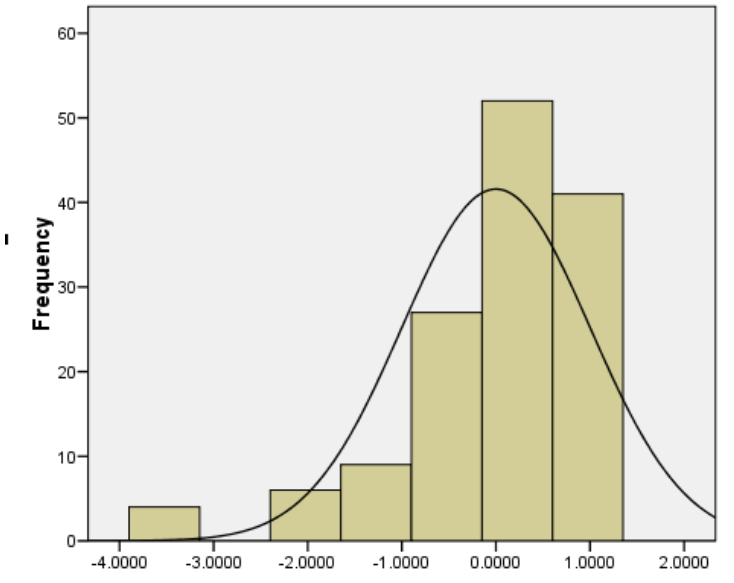

Diagnosis

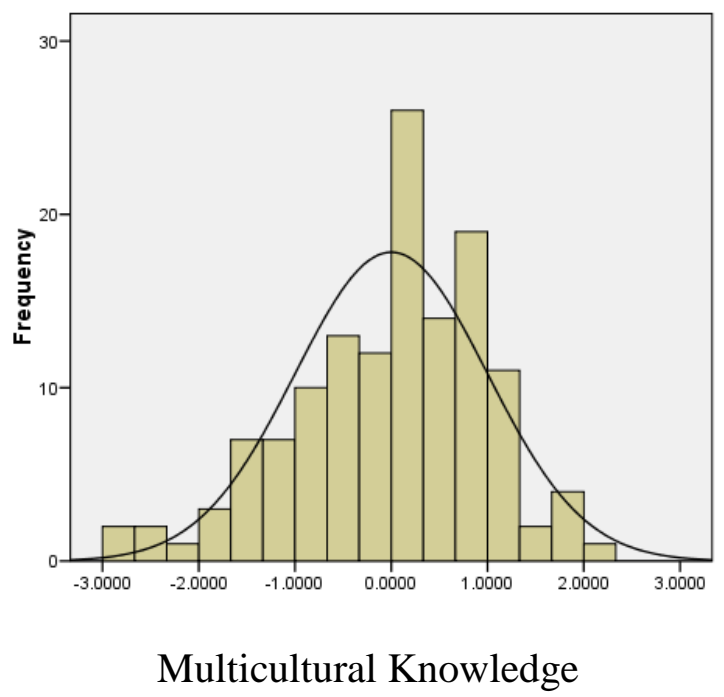

Figure 3. Frequency histograms for each variable 


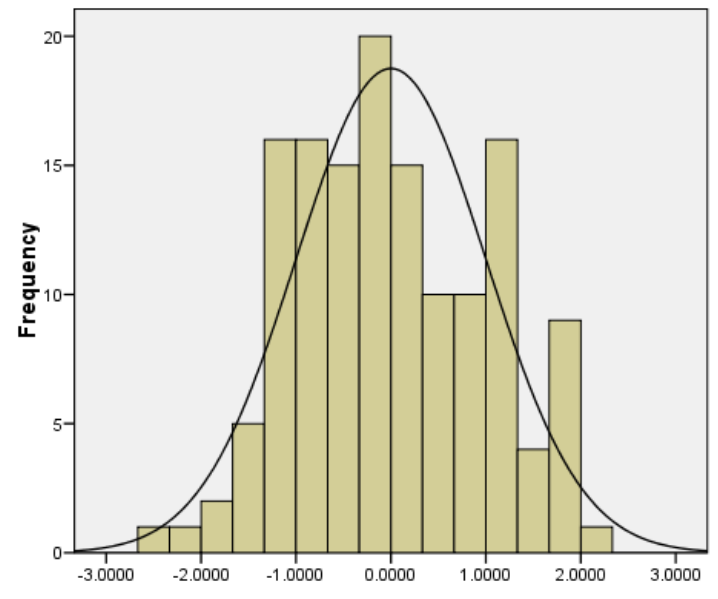

Religiousness

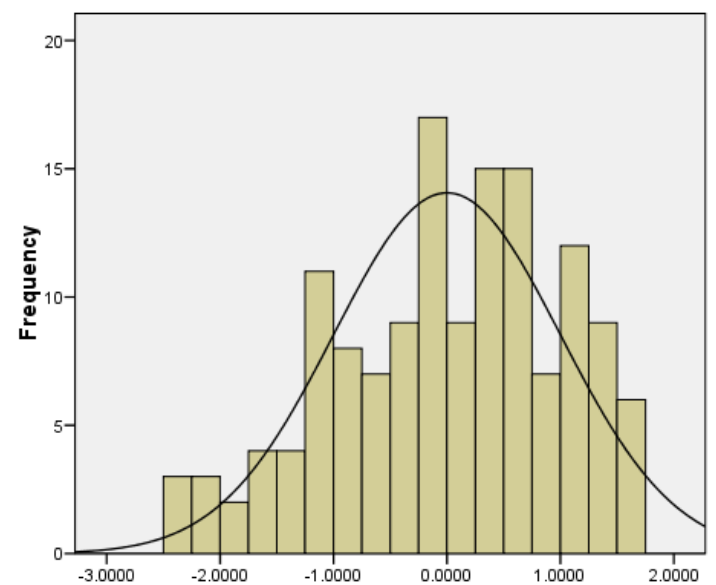

Spirituality

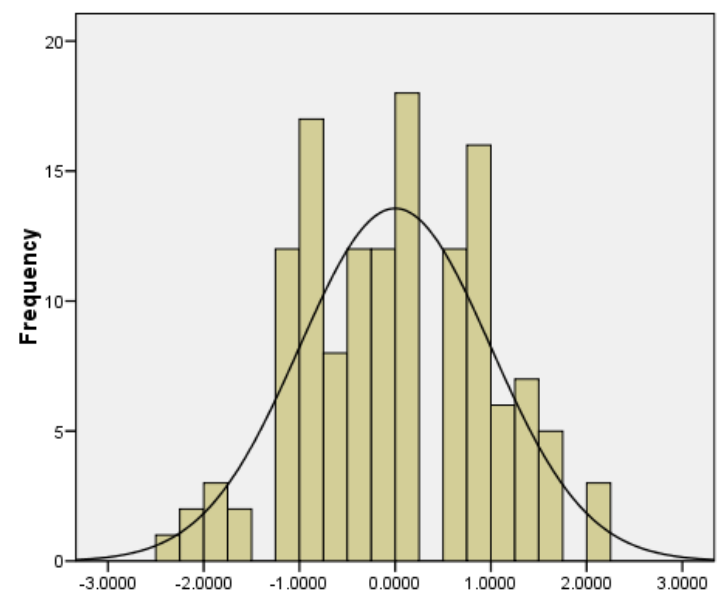

View of Faith Helpfulness

Figure 3, continued. Frequency histograms for each variable 
relationships among all nonmanipulated variables are displayed in Figure 4. A visual inspection of each scatterplot did not reveal any nonlinear relationships between any of the current set of variables.

\section{Heteroscedasticity}

Another assumption of regression is homoscedasticity, the assumption that the variability of scores for a variable remains constant across all levels of another variable to which the original variable is compared (Berry, 1993). Heteroscedasticity refers to the failure of homoscedasticity and occurs when one variable is non-normally distributed or when one variable is related to a transformation of another variable (Tabachnick \& Fidell, 2001). Tabachnick and Fidell (2001) observed that the assumption of homoscedasticity (as well as the assumptions of normality and linearity) may be assessed by examining residuals scatterplots of the dependent variables. Regression standardized predicted values were plotted against regression standardized residuals for Prognosis and Diagnosis in Figure 5. Examination of these scatterplots revealed no evidence of heteroscedasticity in the current data set (as well as no problems with non-normality or nonlinearity).

\section{Outliers}

Outliers refer to observations whose values are unusual or extreme compared to the remaining observations (Fox, 1991; Tabachnick \& Fidell, 2001). Such observations can skew a regression line and lead to misleading results. Like in multicollinearity, there are two kinds of outliers: univariate outliers which have an extreme value on one variable and multivariate outliers which have an extreme combination of scores on two or more variables (Tabachnick \& Fidell, 2001). Univariate outliers can be easily detected through 


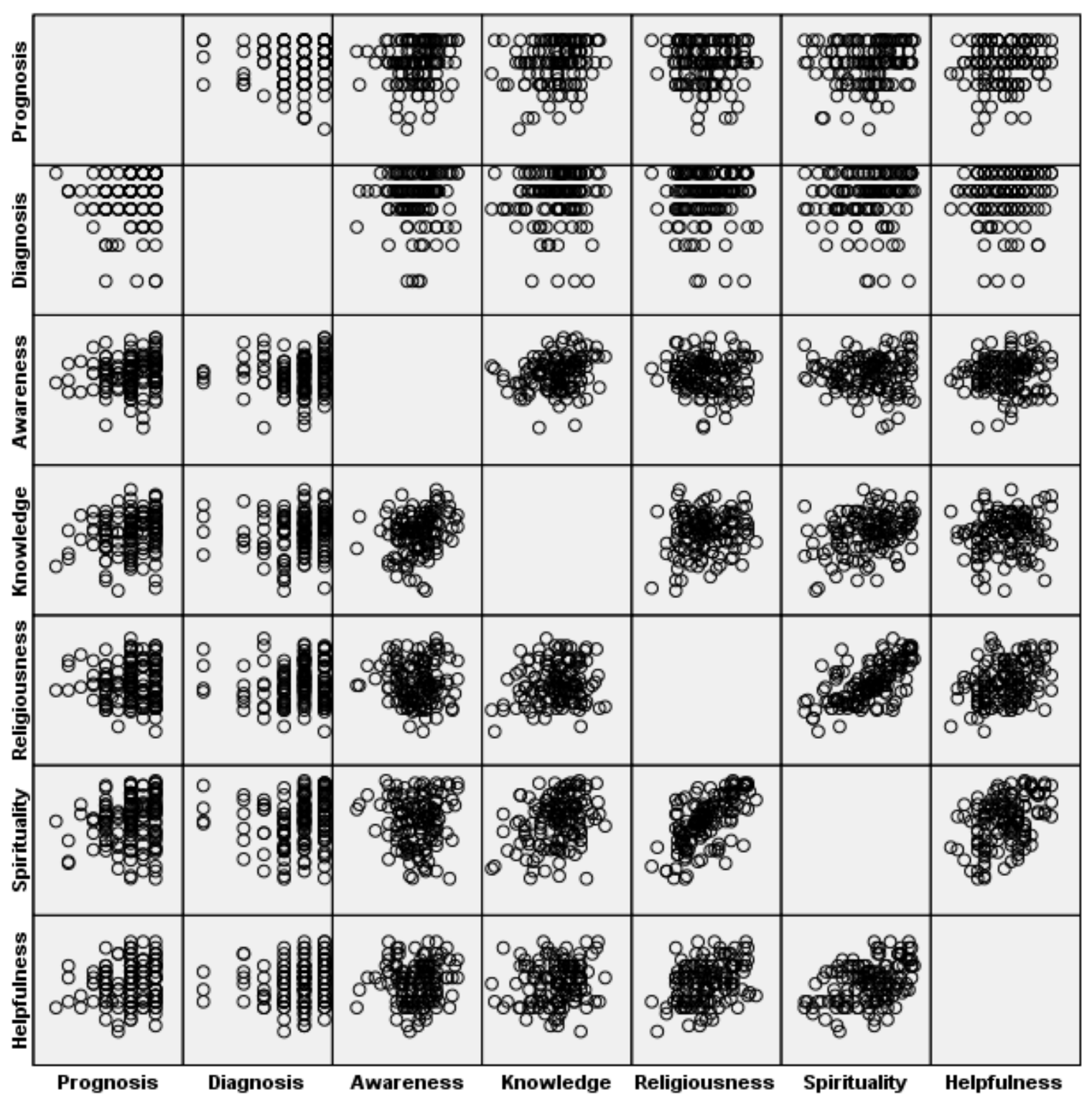

Figure 4. Scatterplots of bivariate relationships among variables 
Dependent Variable: Prognosis

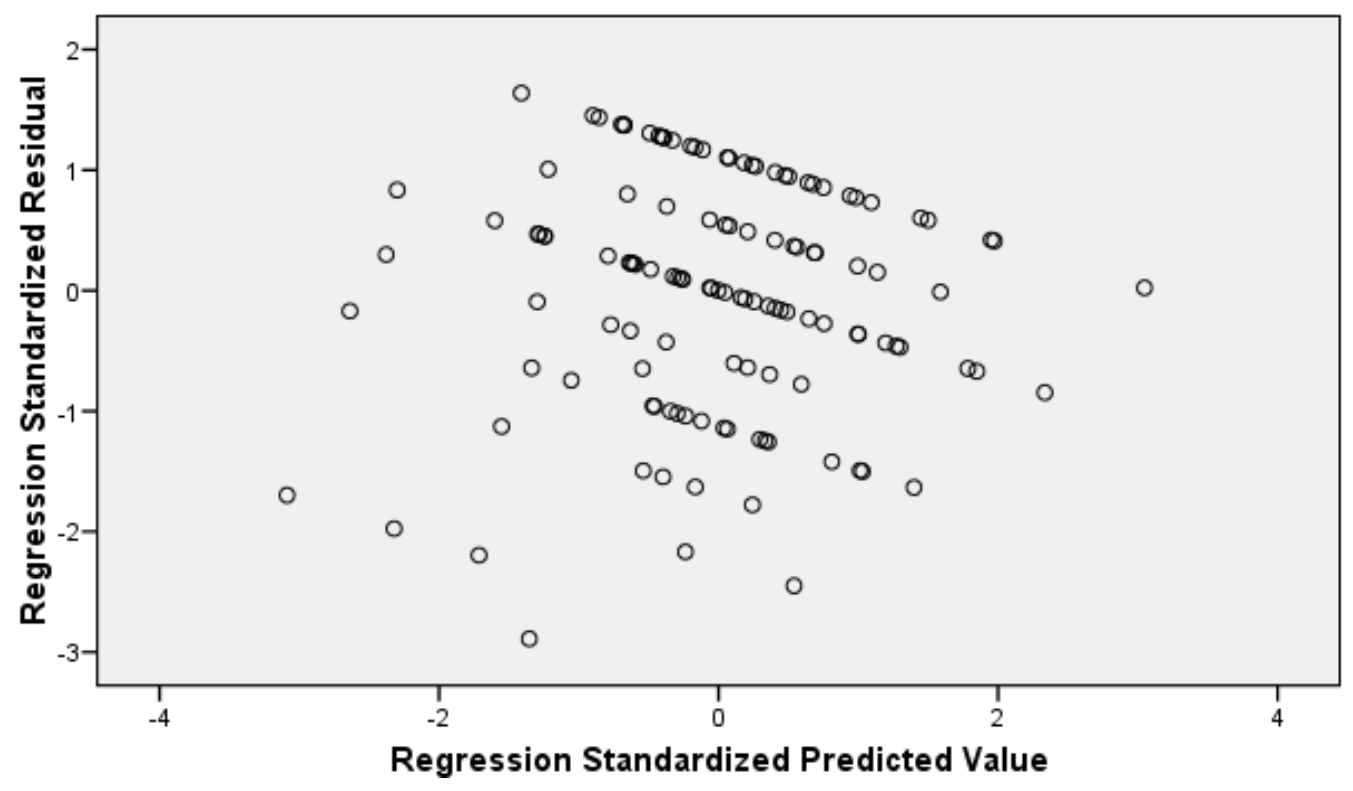

Dependent Variable: Diagnosis

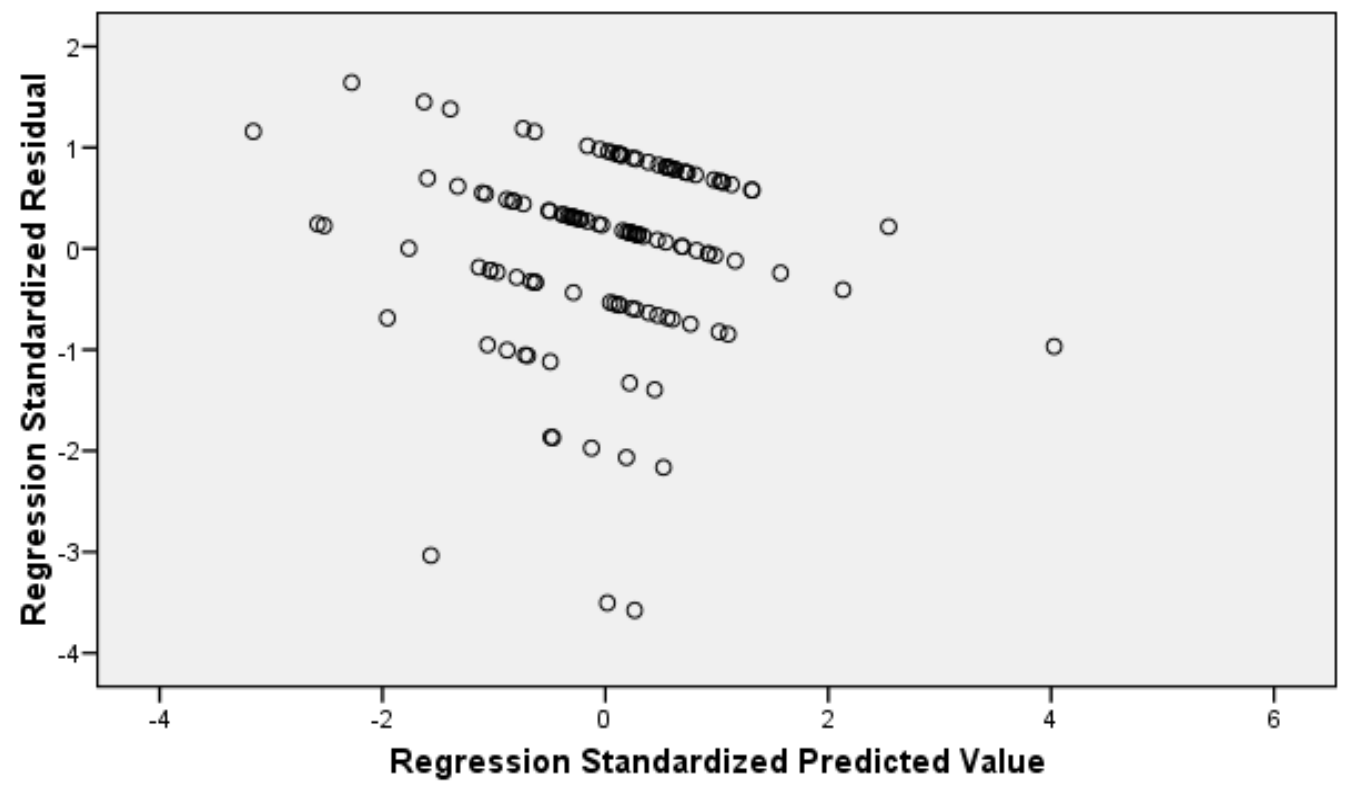

Figure 5. Regression standard predicted values plotted against regression standardized residuals for prognosis and diagnosis 
visual inspection of bivariate scatterplots, while multivariate outliers can be detected by examining Mahalanobis Distances, Leverages, and Cook's D values as well as normal PP plots of regression standardized residuals for extreme cases (Tabachnick \& Fidell, 2001). Scatterplots depicting the bivariate relationships among variables are displayed in Figure 4. The ten highest values for Mahalanobis Distances, Leverage, and Cook's D are displayed in Table 17, while normal P-P plots of regression standardized residuals for Prognosis and Diagnosis are displayed in Figure 6. Visual inspection of Figure 4 reveals no visible univariate outliers, and visual inspection of Figure 6 reveals no visible multivariate outliers. The highest Mahalanobis Distance for both Prognosis and Diagnosis was 16.86, the highest Leverages for Prognosis and Diagnosis were 0.14 and 0.13, respectively, and the highest Cook's D values for Prognosis and Diagnosis were 0.05 and 0.09 , respectively. None of these values were very different from the other values for Mahalanobis Distances, Leverage, and Cook's D, suggesting no observations in the current data set are multivariate outliers. 
Table 17

Ten Highest Values for Mahalanobis Distances, Leverage, and Cook's D

\begin{tabular}{|c|c|c|c|c|c|c|}
\hline DV & Ss & Mahalanobis Distance & Ss & Cook's D & Ss & Leverage \\
\hline \multirow[t]{10}{*}{ Prognosis } & 62 & 46.36 & 74 & 0.11 & 62 & 0.36 \\
\hline & 128 & 44.01 & 102 & 0.11 & 128 & 0.34 \\
\hline & 61 & 43.24 & 62 & 0.11 & 61 & 0.34 \\
\hline & 111 & 37.92 & 118 & 0.10 & 111 & 0.30 \\
\hline & 135 & 37.60 & 111 & 0.09 & 135 & 0.29 \\
\hline & 50 & 32.66 & 60 & 0.07 & 50 & 0.26 \\
\hline & 116 & 30.16 & 85 & 0.06 & 116 & 0.24 \\
\hline & 33 & 27.91 & 61 & 0.06 & 33 & 0.22 \\
\hline & 60 & 27.35 & 46 & 0.06 & 60 & 0.21 \\
\hline & 104 & 26.35 & 49 & 0.05 & 104 & 0.21 \\
\hline \multirow[t]{10}{*}{ Diagnosis } & 62 & 46.36 & 60 & 0.09 & 62 & 0.36 \\
\hline & 128 & 44.01 & 62 & 0.09 & 128 & 0.34 \\
\hline & 61 & 43.24 & 20 & 0.05 & 61 & 0.34 \\
\hline & 111 & 37.92 & 9 & 0.05 & 111 & 0.30 \\
\hline & 135 & 37.60 & 31 & 0.05 & 135 & 0.29 \\
\hline & 50 & 32.66 & 12 & 0.04 & 50 & 0.26 \\
\hline & 116 & 30.16 & 6 & 0.04 & 116 & 0.24 \\
\hline & 33 & 27.91 & 102 & 0.03 & 33 & 0.22 \\
\hline & 60 & 27.35 & 111 & 0.03 & 60 & 0.21 \\
\hline & 104 & 26.35 & 101 & 0.03 & 104 & 0.21 \\
\hline
\end{tabular}


Dependent Variable: Prognosis

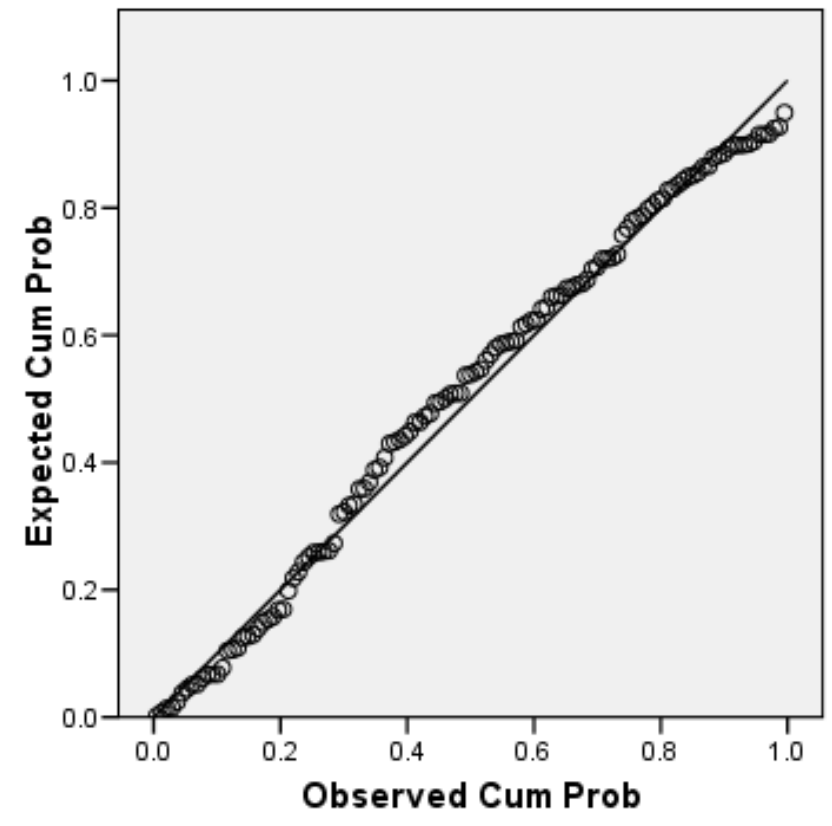

Dependent Variable: Diagnosis

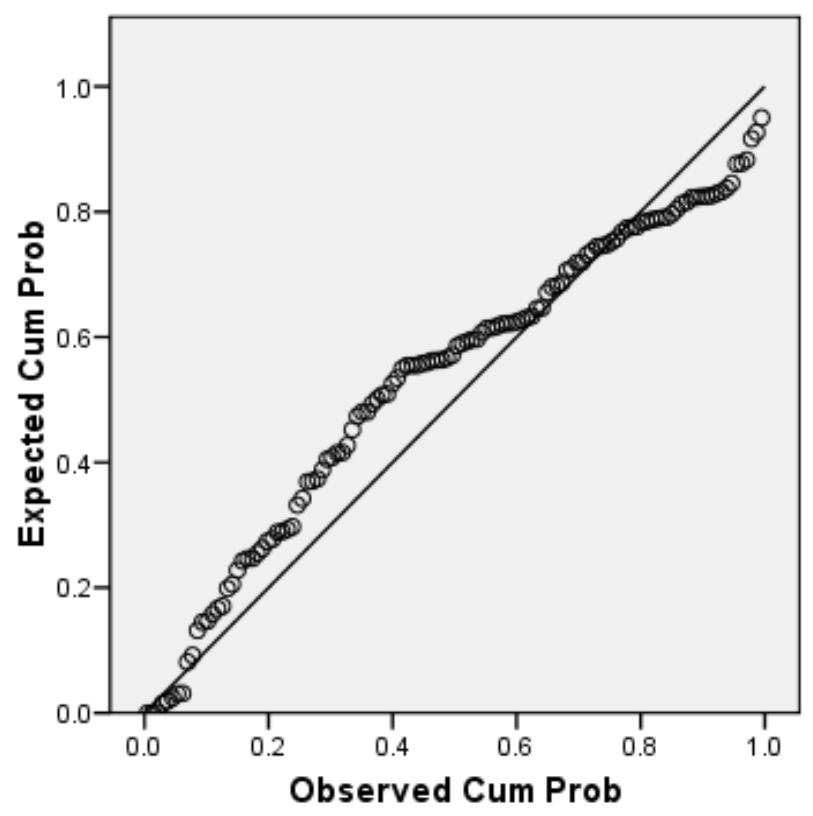

Figure 6. Normal P-P plots of regression standardized residuals for prognosis and diagnosis 